\title{
Nonproliferative and Proliferative Lesions of the Rat and Mouse Endocrine System
}

\author{
Annamaria Brändli-Baiocco ${ }^{1}$, Emmanuelle Balme ${ }^{2}$, Marc Bruder $^{3}$, Sundeep Chandra ${ }^{4}$, Juergen Hellmann 5 , \\ Mark J. Hoenerhoff ${ }^{6}$, Takahito Kambara ${ }^{7}$, Christian Landes ${ }^{1}$, Barbara Lenz ${ }^{1}$, Mark Mense ${ }^{8}$, \\ Susanne Rittinghausen 9 , Hiroshi Satoh ${ }^{10}$, Frédéric Schorsch ${ }^{11}$, Frank Seeliger ${ }^{12}$, Takuji Tanaka ${ }^{13}$, \\ Minoru Tsuchitani ${ }^{14}$, ZBigniew WojCinski ${ }^{15}$, Thomas J. Rosol (CHAIR) ${ }^{16 *}$ \\ ${ }^{I}$ Roche Pharma Research and Early Development, Pharmaceutical Sciences, Roche Innovation Center, Basel, Switzerland \\ ${ }^{2}$ Boehringer Ingelheim Pharma, Biberach an der Riss, Germany \\ ${ }^{3}$ Compugen, Inc., Nonclinical Safety, South San Francisco, California, USA \\ ${ }^{4}$ BioMarin Pharmaceuticals Inc., San Rafael, California, USA \\ ${ }_{5}^{5}$ Merck KGaA, D64293 Darmstadt, Germany \\ ${ }^{6}$ In Vivo Animal Core, Unit for Laboratory Animal Medicine, University of Michigan Medical School, Ann Arbor, Michigan USA \\ ${ }^{7}$ AbbVie, Preclinical Safety, North Chicago, Illinois, USA \\ ${ }^{8}$ Covance, Chantilly, Virginia, USA \\ ${ }^{9}$ Fraunhofer ITEM, Department of Pathology, Hannover, Germany \\ ${ }_{10}^{10}$ wate University, Faculty of Agriculture, Iwate, Japan \\ ${ }^{11}$ Bayer CropScience, Sophia-Antipolis, Cedex, France \\ ${ }_{12}^{2}$ AstraZeneca Pathology, Drug Safety and Metabolism, IMED Biotech Unit, Gothenburg, Sweden \\ ${ }^{13}$ Tohkai Cytopathology Institute, Cancer Research and Prevention, Gifu, Japan \\ ${ }^{14}$ LSI Medience Corporation, Nonclinical Research Center, Ibaraki, Japan \\ ${ }^{15}$ Toxicology \& Pathology Consulting, LLC, Ann Arbor, Michigan, USA \\ ${ }^{16}$ Ohio University, Department of Biomedical Sciences, Athens, Ohio, USA
}

\begin{abstract}
The INHAND (International Harmonization of Nomenclature and Diagnostic Criteria for Lesions in Rats and Mice) Project (www. toxpath.org/inhand.asp) is a joint initiative among the Societies of Toxicological Pathology from Europe (ESTP), Great Britain (BSTP), Japan (JSTP) and North America (STP) to develop an internationally accepted nomenclature for proliferative and nonproliferative lesions in laboratory animals. The purpose of this publication is to provide a standardized nomenclature for classifying microscopic lesions observed in the endocrine organs (pituitary gland, pineal gland, thyroid gland, parathyroid glands, adrenal glands and pancreatic islets) of laboratory rats and mice, with color photomicrographs illustrating examples of the lesions. The standardized nomenclature presented in this document is also available electronically on the internet (http://www.goreni.org/). Sources of material included histopathology databases from government, academia, and industrial laboratories throughout the world. Content includes spontaneous and aging lesions as well as lesions induced by exposure to test materials. A widely accepted and utilized international harmonization of nomenclature for endocrine lesions in laboratory animals will decrease confusion among regulatory and scientific research organizations in different countries and provide a common language to increase and enrich international exchanges of information among toxicologists and pathologists. (DOI: 10.1293/tox.31.1S; J Toxicol Pathol 2018; 31: 1S-95S)
\end{abstract}

Keywords: diagnostic pathology, nomenclature, pituitary, pituicytes, hypophysis, pineal, pinealocytes, thyroid, follicular cells, $\mathrm{C}$ cells, parathyroid, chief cells, adrenal, cortical cells, medullary cells, pancreas, islets, islets of Langerhans

\footnotetext{
*Address correspondence to: Thomas J. Rosol, DVM, PhD, MBA, Department of Biomedical Sciences, Ohio University, 1 Ohio University, Athens, Ohio, 45701, USA.

e-mail: rosolt@ohio.edu

(C2018 The Japanese Society of Toxicologic Pathology

This is an open-access article distributed under the terms of the Creative Commons Attribution Non-Commercial No Derivatives (by-nc-nd)

License $<$ http://creativecommons.org/licenses/by-nc-nd/4.0/>.
}

\section{INTRODUCTION}

The INHAND Project (www.toxpath.org/inhand.asp) is a collaboration among the Societies of Toxicologic Pathology from Europe (ESTP), Great Britain (BSTP), Japan (JSTP) and North America (STP) to develop internationally accepted nomenclature for proliferative and nonproliferative lesions in laboratory animals. The purpose of this publication is to provide a standardized nomenclature for classifying proliferative and nonproliferative lesions observed in the endocrine sys- 
tem of laboratory rats and mice. Standardized nomenclature of proliferative and nonproliferative endocrine lesions in rats has been previously published by the STP (Botts et al. 1991; Frith et al. 2000; Majka et al. 1990; Patterson et al. 1995; Riley et al. 1990b). The standardized nomenclature of proliferative endocrine lesions presented in the current document is also available electronically at the goRENI website on the internet (www.goreni.org). In this document, the endocrine system is divided into pituitary, pineal, thyroid (follicular and C-cells), parathyroid, adrenal (cortex and medulla), and pancreatic islets of Langerhans and nonproliferative and proliferative lesions are described for each organ. This document contains spontaneous and aging lesions as well as lesions induced by exposure to test materials.

\section{Pituitary Gland}

The pituitary gland (hypophysis) may be divided into two major compartments: (1) the adenohypophysis (anterior lobe) composed of the pars distalis, pars tuberalis, and pars intermedia; and (2) the neurohypophysis (posterior lobe), which includes the pars nervosa and infundibulum. The pars intermedia is separated from the pars distalis by Rathke's cleft, which is a remnant of the lumen of the craniopharyngeal duct. The pituitary lies within the sella turcica of the sphenoid bone. A hypothalamic-hypophyseal portal blood system transports hypothalamic-releasing and release-inhibiting hormones directly to the adenohypophysis for interactions with their specific target cells (Rosol et al. 2013).

The neurohypophysis is joined to the hypothalamus via the infundibular stalk and is composed of densely packed bundles of unmyelinated axons and capillaries that are supported by modified glial cells or pituicytes. The capillaries in the pars nervosa are termination sites for unmyelinated axons, which originate from the hypothalamic neurosecretory neurons. Axons arising from supraoptic and paraventricular nuclei of the hypothalamus terminate in the pars nervosa. Both oxytocin and vasopressin (antidiuretic hormone, ADH) are synthesized in supraoptic and paraventricular nuclei as large precursor molecules, which contain both active hormones and their associated neurophysins (carrier proteins which transport the hormones). As the biosynthetic precursor molecules travel along the axons in secretion granules from the neurosecretory neurons, the precursors are cleaved into the active hormones and their respective neurophysins.

The pars distalis of the adenohypophysis represents the largest portion of the pituitary gland. The cells within this lobe are responsible for the synthesis of at least six major hormones: growth hormone $(\mathrm{GH})$, prolactin (PRL), and adrenocorticotropic hormone (ACTH), follicle-stimulating hormone (FSH), luteinizing hormone ( $\mathrm{LH})$, and thyroid-stimulating hormone (TSH) or thyrotropin. The GH ( $\sim 50 \%$ of the pars distalis cells) and PRL-secreting cells $(\sim 15-25 \%$ of the pars distalis cells) stain as acidophils and contain abundant cytoplasmic secretory granules. Some GH cells may be chromophobic if they are in an actively synthesizing phase of the secretory cycle. Pregnan- cy and lactation are associated with hyperplasia of prolactinsecreting cells. These so called "pregnancy cells" are chromophobic by routine staining techniques but contain prominent arrays of granular endoplasmic reticulum, well-developed Golgi complexes, and few secretory granules. ACTH-producing cells (corticotrophs) consist of $\sim 15 \%$ of the pars distalis cells. The cells are round to ovoid, may be chromophobic or lightly basophilic, and can stain weakly positive with PAS. Crooke's hyaline change refers to the intracellular accumulation of an eosinophilic homogeneous material within the cytoplasm of these cells. Immunohistochemical staining reveals that this material represents a keratin-like protein, which accumulates in conditions associated with glucocorticoid hormone excess.

Thyrotropin-producing (TSH) basophils or thyrotrophs ( $\sim 5 \%$ of the pars distalis cells) tend to occur in small clusters within the pars distalis and have a stellate to polygonal shape. They may be basophilic or chromophobic and are also PAS-positive. Any condition that leads to hypofunction of the thyroid or increased hepatic degradation of thyroid hormones, results in the development of "thyroidectomy cells" in the pituitary gland. "Thyroidectomy cells" are enlarged and vacuolated TSH cells that contain prominent, often dilated granular endoplasmic reticulum, conspicuous Golgi complexes, and few secretory granules. Gonadotropin-producing (GTH) basophils or gonadotrophs ( $\sim 10 \%$ of the pars distalis cells) are relatively large round to oval cells, which are responsible for the production of FSH and LH. Gonadotroph cells undergo a series of changes following castration, resulting in the formation of "gonadectomy cells". As a result of the lack of negative feedback by gonadal steroids, gonadotrophs are actively stimulated to synthesize and secrete FSH and LH. Gonadotrophs undergo hypertrophy, and the cytoplasm becomes vacuolated due to distention profiles of endoplasmic reticulum with finely granular material in response to long-term stimulation by gonadotropin-releasing hormone $(\mathrm{GnRH})$ from the hypothalamus. In some cells, a single large vacuole occupies most of the cytoplasm, producing cells with a "signet ring" appearance. The pars intermedia consists of chromophobic cells that is readily identified by its location. These endocrine cells of the pars intermedia produce melanocyte-stimulating hormones $(\alpha-\mathrm{MSH}$ and $\beta$-MSH), ACTH, and other peptide hormones.

In addition to specific hormone-secreting cells, a population of supporting cells is also present in the adenohypophysis. These cells have been referred to as stellate (follicular) cells and can be stained selectively with antibodies to S-100 protein or cytokeratin. Stellate cells typically have elongate processes and prominent cytoplasmic filaments. These cells provide a phagocytic or supportive function in addition to producing a colloid-like material.

The different endocrine cells of the adenohypophysis are not uniformly distributed. The regions that contain acidophils (acidophil zones) are conspicuous because there are more acidophils than other cell types and the cytoplasm stains intensely. The other zones contain a mixture of basophils and chromophobic endocrine cells, and the chromophobic cells predominate. Basophils often occur in small clusters admixed with 
chromophobic cells. The most efficient way to accurately identify endocrine cell types is to use immunohistochemistry for secretory hormones. The staining intensity will vary depending on the amount of stored hormone in the cytoplasm. Hypertrophied endocrine cells with a high rate of hormone synthesis and secretion will stain lightly using immunohistochemistry due to the low number of cytoplasmic secretory granules.

The hypothalamus serves as the major regulator of the adenohypophysis. Each cell type within the adenohypophysis is under the control of a corresponding releasing hormone that is synthesized within nerve cell bodies of the hypothalamus. The releasing hormones are transported via axonal processes to the median eminence where they are released into capillaries and are carried by the hypophyseal portal system to trophic hormone-producing cells in the adenohypophysis. Specific releasing factors have been identified for TSH, FSH and $\mathrm{LH}$, ACTH, and GH. PRL secretion is stimulated by a number of factors, the most important of which appears to be thyrotropin releasing hormone (TRH).

Multiple influences contribute to the control of adenohypophyseal hormone secretion. Dopamine serves as the major prolactin inhibitory factor. Dopamine also suppresses ACTH and melanocyte stimulating hormone production by corticotrophs in the pars intermedia. A second hypothalamic release inhibiting hormone is somatostatin (somatotropin release-inhibiting hormone, SRIH). This tetradecapeptide inhibits the secretion of both GH and TSH. In some situations, SRIH also inhibits the secretion of PRL and ACTH. The control of pituitary hormone secretion is also affected by negative feedback loops resulting from the interaction of end organ hormones, adenohypophyseal hormones, and corresponding hypothalamic releasing-and release-inhibiting hormones. Estrogen stimulates prolactin secretion and lactotroph proliferation, particularly in the rat.

\section{Pineal Gland}

The pineal gland is part of the brain and functions as a neuroendocrine organ. Its principle secretion is melatonin. The gland is located between the occipital poles of the telencephalon and the cerebellum. The gland has a superficial and deep portion connected by a stalk, and is close to the third ventricle and subarachnoid space. Calcareous concretions are common, which increase with age and apparently do not affect function of the gland. The pineal gland is composed of pinealocytes, neurons, and supporting glial cells with blood vessels. The gland is surrounded by a capsule. The pinealocytes are derivatives from pineal photoreceptors that occur in lower species, such as poikilotherms, and the cells may contain ultrastructural remnants of the photoreceptor cells. The pinealocytes produce and secrete melatonin and also contain serotonin. Dense-core secretory granules are sparse compared to other endocrine cells. The neurons of the pineal gland connect to the central nervous system and the gland is also innervated by the sympathetic system.

\section{Thyroid Gland}

The thyroid gland originates as a thickened plate of epithelium in the floor of the pharynx to form the thyroglossal duct, which extends along the midline to the region of the larynx in the fetus. The paired lobes of the thyroid form from the thyroglossal duct on either side of the larynx and proximal trachea. The ultimobranchial bodies fuse with the thyroid and deliver $\mathrm{C}$ cells (neural crest origin) to each thyroid lobe. Accessory thyroid tissue may form from small remnants of the thyroglossal duct from the larynx to the heart, but this is rare in rodents. Accessory thyroid tissue can be identified by its general lack $\mathrm{C}$ cells. Also rare in rodents are thyroglossal duct cysts, which develop in the ventral aspect of the anterior cervical region and occur as a result of persistence of a portion of the thyroglossal duct postnatally.

The basic structure of the thyroid gland is unique for endocrine glands, consisting of follicles of varying size (20-250 $\mu \mathrm{m})$ that contain colloid produced by the follicular cells. Follicular cells are cuboidal to columnar, and their secretory polarity is directed toward the lumen of the follicles. The biosynthesis of thyroid hormones is also unique among endocrine glands because the final assembly of the hormones occurs extracellularly within the follicular lumen. The assembly of thyroid hormones within the follicular lumen is made possible by a unique protein (thyroglobulin) synthesized by follicular cells. Thyroglobulin is a high molecular weight $(600,000$ to 750,000$)$ glycoprotein. The amino acid tyrosine, an essential component of thyroid hormones, is incorporated in the molecular structure of thyroglobulin. Iodine is bound to tyrosyl residues in thyroglobulin at the apical surface of follicular cells to form, successively, monoiodotyrosine (MIT) and diiodotyrosine (DIT). The resulting MIT and DIT combine to form the two biologically active iodothyronines-thyroxine, $\mathrm{T}_{4}$, and triiodothyronine, $\mathrm{T}_{3}$-secreted by the thyroid gland. The mechanism of active transport of iodide is associated with a sodium iodide $\left(\mathrm{Na}^{+} \mathrm{I}^{-}\right)$ symporter (NIS). The transporter protein is in the basolateral membrane of thyroid follicular cells (thyrocytes).

Long-term perturbations of the pituitary-thyroid axis by various xenobiotics or physiologic alterations (e.g., iodine deficiency or goitrogens) are likely to predispose the laboratory rat to a higher incidence of proliferative lesions (e.g., hyperplasia and adenomas of follicular cells) in response to chronic TSH stimulation than in the human thyroid. This is particularly true in the male rat, which has higher normal circulating concentrations of TSH than females. The greater sensitivity of the rodent thyroid to derangement by drugs, chemicals, and physiologic perturbations is also related to the shorter plasma half-life of $\mathrm{T}_{4}$ than in man due to the considerable differences between species in the transport proteins for thyroid hormones. The plasma $\mathrm{T}_{4}$ half-life in rats is considerably shorter (12-24 hr.) than in man (5-9 days). In human beings and monkeys circulating $T_{4}$ is bound primarily to thyroxine-binding globulin (TBG) but this high-affinity binding protein is not present in rodents. Mice are less susceptible to proliferative lesions of the thyroid follicular cells when compared to rats. 
Negative feedback control of thyroid hormone secretion is accomplished by the coordinated response of the adenohypophysis and certain hypothalamic nuclei to circulating and local tissue levels of $\mathrm{T}_{3}$. In the rat, $50 \%$ or more of the pituitary content of $T_{3}$ is generated locally from circulating $T_{4}$ by a 5'-deiodinase (type II). A decrease in thyroid hormone concentration in plasma is sensed by neurosecretory neurons in the hypothalamus that synthesize and secrete thyrotropin releasing hormone (TRH; $361 \mathrm{Da}$ ) into the hypophyseal portal circulation. TRH binds to receptors on the plasma membrane of thyrotrophic basophils in the adenohypophysis, which leads to release of TSH-containing secretory granules into pituitary capillaries. Thyroid-stimulating hormone is conveyed to thyroid follicular cells where it binds to the basilar aspect of the cell and increases the rate of the synthesis and secretion of thyroid hormones. If the secretion of TSH is sustained (hours or days), thyroid follicular cells become more columnar and follicular lumens become smaller due to the increased endocytosis of colloid. Numerous PAS-positive colloid droplets are present in the luminal aspect of the hypertrophied follicular cells. Conversely, in response to an increase in circulating levels of thyroid hormones $\left(\mathrm{T}_{4}\right.$ and $\left.\mathrm{T}_{3}\right)$, there is a corresponding decrease in circulating pituitary TSH. Thyroid follicles become enlarged and distended with colloid due to the decreased TSH-mediated endocytosis of colloid.

Thyroid hormones are degraded primarily by conjugation in the liver. Thyroxine is conjugated on the outer phenolic ring with glucuronic acid in a reaction catalyzed by thyroxine UDP glucuronosyltransferase, and conjugated $\mathrm{T}_{4}$ is excreted in the bile. A wide variety of drugs and chemicals can influence thyroid hormone metabolism by inducing one or more classes of hepatic microsomal enzymes that increase the degradation of thyroid hormones. The stepwise monodeiodination of thyroxine in the liver, kidney, and elsewhere is also important in the metabolism of thyroid hormones. The removal of an iodine molecule from the 5 position on the outer phenolic ring by 5 'deiodinase results in the formation of biologically active $\mathrm{T}_{3}$ (3,5,3'-triiodothyronine). However, if a molecule of iodine is removed from the 5 position of the inner phenolic ring of $\mathrm{T}_{4}$ by another enzyme, 5-deiodinase, reverse $\mathrm{T}_{3}\left(3,3^{\prime}, 5^{\prime}\right.$-triiodothyronine) is produced, which is biologically inactive.

The subcellular mechanism of action of thyroid hormones resembles that of steroid hormones because free hormone enters into target cells and binds to its receptors. Free $T_{3}$ either binds to receptors on the inner mitochondrial membrane to activate mitochondrial energy metabolism or binds to nuclear receptors and increases transcription of mRNA to facilitate new protein synthesis. The overall effects of thyroid hormones are to (1) increase the basal metabolic rate; (2) increase glycolysis, gluconeogenesis, and glucose absorption from the intestine; (3) stimulate new protein synthesis; (4) increase lipid metabolism and conversion of cholesterol into bile acids and other substances, activate lipoprotein lipase, and increase the sensitivity of adipose tissue to lipolysis by other hormones; (5) stimulate the heart rate, cardiac output, and blood flow; and (6) increase neural transmission, cerebration, and neuronal development in young animals.

C cells or parafollicular cells of the thyroid gland are located within thyroid follicles between the basal aspects of the follicular cells and the basement membrane of the follicle or are present in a parafollicular position. In addition to calcitonin, C-cells contain a variety of other peptides, including calcitonin gene-related peptide and somatostatin, and they are also positive for a wide variety of generic neuroendocrine markers, including chromogranins and synaptophysin. In most rats and mice, C-cells are concentrated within the central regions of the lobes, being most prominent at the levels of the parathyroid glands. By light microscopy, C-cells often have a clear or light appearance, but silver-positive cytoplasmic granules can be demonstrated by argyrophilic staining sequences. $\mathrm{C}$ cells are more easily identified in rats compared to mice because they have greater numbers of $\mathrm{C}$ cells that also increase with age. Immunohistochemistry for calcitonin can be helpful for evaluation of $\mathrm{C}$ cell numbers, particularly in mice.

Calcitonin is a 32-amino acid peptide that is derived from a 141-amino acid precursor (procalcitonin). The levels of ionized calcium in plasma and extracellular fluids are the major physiologic stimulus for the secretion of calcitonin. The cell membrane calcium-sensing receptor, cloned from parathyroid cells, is also expressed in C-cells and contributes to the regulation of calcitonin secretion. Calcitonin interacts with specific receptors in target cells, principally in bone and kidney. Calcitonin inhibits osteoclast activity in bone, renal tubular resorption of calcium, and calcium absorption by the intestines. Calcitonin secretion is increased in response to a high calcium meal often before a significant rise in plasma calcium levels can be detected. The secretion of gastrin, cholecystokinin and glucagon is stimulated by an oral calcium load, and these hormones also serve as calcitonin secretagogues. Calcitonin also protects against calcium loss from the skeleton during periods of calcium mobilization, such as growth, pregnancy, and lactation.

\section{Parathyroid Glands}

The paired parathyroid glands are usually located on the anterior and lateral aspect of the thyroid lobes, and are separated from the thyroid by a thin capsule of fibrous connective tissue. Embryologically, parathyroids are of endodermal origin, derived from the pharyngeal pouches in close association with the primordia of the thymus. Accessory parathyroid tissue can occur in the thymus or dorsolateral to the esophagus near the larynx. The mass of the parathyroid glands of female rats is up to twice that of males.

Parathyroids contain a single type of secretory (chief) cell that synthesizes and secretes parathyroid hormone (PTH). Parathyroid glands are composed of chief cells in different stages of secretory activity. Oxyphil cells occur in certain animal species and humans, but do not occur in rats and mice. Oxyphil cells are larger than chief cells and their abundant eosinophilic cytoplasm is filled with numerous large, often bizarre-shaped, mitochondria.

The parenchyma of the parathyroid glands consists of 
densely packed, highly folded, branching cords or clusters of polygonal cells separated by a delicate stroma of reticular and collagen fibers with occasional fibrocytes (Mense and Rosol 2018b). The cords are usually a single cell layer in a trabecular and acinar arrangement. The chief cells are uniform with spherical to oval to elongated nuclei and scant to moderate amounts of cytoplasm. The nuclei have loosely aggregated or finely dispersed chromatin and inconspicuous nucleoli. The cytoplasm is faintly eosinophilic. The amount of cytoplasm usually reflects the level of activity of the chief cells. Chief cells with increased synthesis and secretion of parathyroid hormone are hypertrophied with increased cytoplasm and reduced eosinophilia or vacuolation of the cytoplasm.

PTH is involved in the regulation of calcium homeostasis with calcitonin and calcitriol (the active form of vitamin D). The principal action of PTH is to maintain adequate blood calcium levels by controlling the rate of calcium resorption from bone, enhancing the absorption of calcium from the renal tubules and stimulating active absorption of calcium from the intestine (via calcitriol). The chief cells store relatively small amounts of preformed hormone but respond to minor fluctuations in blood calcium ion, and to a much lesser extent magnesium ion, by rapidly altering the rate of hormonal secretion and degradation and more slowly altering the rate of synthesis. Hypocalcemia causes the rapid release of PTH into the blood, which increases the bone reabsorbing activity of osteocytes and osteoclasts. PTH also has a rapid and direct effect on renal proximal tubules, blocking the reabsorption of phosphate as well as enhancing the absorption of calcium from the distal convoluted tubule. The rising levels of calcium ion in the plasma inhibit the secretion of PTH (negative feedback inhibition). PTH also stimulates and regulates the conversion of 25-hydroxycholecalciferol to 1,25-dihydroxycholecalciferol (calcitriol) in the kidney, which is required for PTH to regulate the activity of osteocytes and osteoclasts and for calcium absorption from the intestine. Rising plasma levels of calcitriol provide additional feedback regulation by inhibiting further secretion of PTH.

\section{Adrenal Cortex and Medulla}

The adrenal glands are the most commonly affected endocrine organs secondary to chemical exposure (Rosol et al. 2001). In the adrenal glands, chemically induced lesions are found most frequently in the zona fasciculata and reticularis and to a lesser extent in either the zona glomerulosa or the medulla.

The adrenal glands are located close to the anterior pole of the kidneys. They receive arterial blood from branches of the aorta or from regional arteries that result in a vascular plexus, and perfusion occurs by sinusoids that perfuse the entire gland, including both the cortex and the medulla. Venous blood flow is derived from the sinusoidal network with eventual flow into the medulla.

Grossly, a midsagittal section of the adrenal glands reveals a clear separation between the cortex and the medulla. The cor- tex is yellow, and occupies approximately two-thirds of the entire cross-sectional diameter of the organ. Cortical zones (from outer to inner) consist of the zona glomerulosa, zona fasciculata, and zona reticularis. These zones are not always clearly delineated, and the zona reticularis is not morphologically delineated in the mouse. The mineralocorticoid-producing zona glomerulosa contains cells aligned in a sigmoid pattern in relationship to the capsule. Loss of this zone or the inability to secrete mineralocorticoids (e.g., aldosterone) may result in death of the animal due to the retention of inappropriately high levels of potassium in association with an excessive loss of sodium chloride and water. The largest zone is the zona fasciculata ( $>70 \%$ of the cortex). Cells in this zone are arranged in long anastomosing cords or columns, separated by small capillaries. They are responsible for the secretion of glucocorticoid hormones (e.g., corticosterone in the rat and mouse).

The adrenal cortical cells contain large cytoplasmic lipid droplets, which consist of cholesterol and other steroid precursors. The lipid droplets are in close proximity to the smooth endoplasmic reticulum and large mitochondria, which contain the specific hydroxylase and dehydrogenase enzyme systems required to synthesize the different steroid hormones. Unlike polypeptide hormone-secreting cells, there are no secretory granules in the cytoplasm because there is direct secretion without significant storage of preformed steroid hormones.

Adrenal steroids are synthesized from cholesterol, which is derived from acetate or circulating lipoproteins. A complex shuttling of steroid intermediates between mitochondria and endoplasmic reticulum characterizes specific synthetic processes. The specificity of mitochondrial hydroxylation reactions in terms of the steroid modified and the position of the substrate that is hydroxylated are confined to a specific cytochrome P450 (CYP). Corticosterone is the major glucocorticoid produced in rats and mice. Essentially, rodents lack CYP17 and this is an important consideration for toxicology, as compounds that inhibit this enzyme may not be fully detected in rodent species. Species with CYP17 produce cortisol and those lacking CYP17 produce corticosterone as the major glucocorticoid. CYP17 is required for androgen production by the zona reticularis, therefore, rats and mice synthesize no to minimal sex steroids.

The principal control for the production of steroids by the zona fasciculata and zona reticularis is mediated by adrenocorticotrophic hormone (adrenocorticotropin; ACTH) produced by corticotrophs in the adenohypophysis. ACTH release is largely controlled by the hypothalamus through the secretion of corticotropin-releasing hormone $(\mathrm{CRH})$ and arginine-vasopressin. An increase in ACTH production normally results in an increase in circulating levels of glucocorticoids, although it can cause weak stimulation of aldosterone secretion as well. Negative feedback control normally occurs when the elevated blood levels of cortisol act on the hypothalamus, anterior pituitary, or both to cause a suppression of ACTH secretion.

The adrenal cortex is dependent on trophic support of hormones from the pituitary and hypothalamus, as well as, hormones from other endocrine tissues. Additionally, the adrenal 
cortex has both anatomic and molecular characteristics that convey vulnerability to toxic insult (Rosol et al. 2013; Rosol et al. 2001).

The adrenal medulla constitutes approximately $10 \%$ of the volume of the adrenal gland. Histologically, the normal adrenal medulla in the rodent is sharply demarcated from the surrounding cortex. The bulk of the medulla is composed of chromaffin cells, which are the sites of synthesis and storage of catecholamines. In the rat and mouse, norepinephrine and epinephrine are stored in separate chromaffin cell types, which can be distinguished ultrastructurally by the morphology of their secretory granules. In addition to chromaffin cells, the adrenal medulla contains variable numbers of ganglion cells. A third cell type has also been described and has been designated the small granule-containing (SGC) cell or small intensely fluorescent (SIF) cell. These cells morphologically appear intermediate between chromaffin cells and ganglion cells, and are thought to possibly function as interneurons.

\section{Endocrine Pancreas (Islets of Langerhans)}

The endocrine pancreas consists of discrete aggregates of cells distributed throughout the pancreas, called the islets of Langerhans. The islets comprise approximately $1-2 \%$ of the total pancreatic tissue in a normal adult rat. The main function of the endocrine pancreas is the regulation of blood glucose. Diabetes mellitus (DM) is the clinical condition where there is failure of the control of blood glucose with the development of hyperglycemia and hyperglucosuria. While lesions in the islets of Langerhans are infrequently observed in toxicity studies, the mouse and rat have been important for studying the pathogenesis of diabetes mellitus resulting from obesity, genetic predisposition, autoimmune disease, or chemically induced islet cell injury (Mense and Rosol 2018a; Rosol et al. 2013).

The pancreas is formed from two distinct outgrowths of endoderm located in the foregut at the level of the duodenum. The larger dorsal anlage and smaller ventral bud fuse to form the dorsal and ventral portions of the pancreas. The islets are formed from a progenitor cell population located in the small ducts of the pancreatic primordium from which the pancreatic acinar cells arise. Clusters of immunoreactive endocrine cells recognized as primitive islets are present by day 14 of gestation. As the fetal islets develop, a proportion of them detach from the duct system. $\alpha$ and $\beta$ cell numbers increase rapidly during late fetal life, and they make up a majority of the islet cell population at birth.

Pancreatic endocrine cells are arranged in small aggregates, the islets of Langerhans, or are located individually or in small clusters in contact with acini or ducts. Islets of Langerhans range from 100 to $200 \mu \mathrm{m}$ in diameter in adult rats, and consist of closely apposed branching cords of pale-staining polygonal cells surrounded by a basal lamina, a delicate network of reticulum fibers, and frequent interspersed capillaries. A fibrous tissue capsule is not present. The cells are homogeneous with finely granular, lightly eosinophilic cytoplasm and round to oval nuclei containing finely stippled chromatin and a single nucleolus. The nuclei are usually located opposite the secretory pole which is adjacent to the capillary. Multiple cell types, each capable of secreting one or more hormones, are present in the pancreatic islets. These cells, which contain glucagon ( $\alpha$ cells), insulin ( $\beta$ cells), somatostatin ( $\delta$ cells), pancreatic polypeptide (PP or F cells), substance P (enterochromaffin cells), or ghrelin ( $\varepsilon$ cells) are not randomly distributed in the islets, but rather are located in specific areas. In rodents, the $\alpha$ cells, $\delta$ cells, and less numerous PP cells occur at the periphery of the islet, whereas the $\beta$ cells are located in the center and are the most numerous, comprising about $60-80 \%$ of the islet volume. The $\alpha$ cells and $\delta$ cells comprise approximately $2-28 \%$ and $<10 \%$ of the islet cells, respectively (Steiner et al. 2010). The individual cell types within the islets of Langerhans are differentiated most readily by electron microscopy or immunohistochemical techniques, since the hormone-containing secretory granules are unique biochemically and structurally. The blood vessels of the islets are lined with fenestrated endothelium. Some islet capillaries also supply pancreatic acinar cells in the peri-islet zone. The arrangement of the microvasculature may be important for the paracrine activity of islet hormones.

The islets in the dorsal and ventral portions of the pancreas arise from different anlagen and contain different cell populations. Islets in the tail, body, or superior part of the head of the pancreas (collectively the dorsal or "splenic" part of the pancreas) contain a greater proportion of glucagon-containing $\alpha$ cells $(\sim 28 \%)$ than PP cells $(2 \%)$. The reverse is true in the middle and inferior part of the head (ventral or "duodenal" region with $\sim 2 \% \alpha$ cells and $20 \%$ PP cells).

The various islet hormones are involved in regulation of multiple metabolic activities. Insulin accounts for approximately $85 \%$ of the hormone production of the endocrine pancreas, and has the primary function of facilitating entry of glucose through cell membranes. In addition, it influences glucose utilization by controlling gluconeogenesis in liver, muscle, and adipose tissue. By these dual mechanisms, insulin maintains blood glucose levels within the appropriate physiological range. Glucagon promotes glucose mobilization by stimulating hepatic glycogenolysis and gluconeogenesis from amino acids and fatty acids. Somatostatin, named for its action in suppressing secretion of growth hormone, inhibits secretion of insulin and glucagon. Control of the release of islet cell hormones is influenced by four major mechanisms: (1) blood levels of nutrients including glucose, fatty acids, and amino acids; (2) postprandial secretion of incretin hormones (such as glucagon-like peptide-1, GLP-1, and gastric inhibitory peptide, GIP) from enteric endocrine cells that stimulate glucose-dependent insulin secretion; (3) activity of the autonomic nervous system (parasympathetic stimulation favors secretion of both insulin and glucagon, whereas sympathetic activity inhibits insulin release and promotes glucagon secretion); and (4) paracrine activity of islet hormones on neighboring cells. By this latter mechanism insulin inhibits glucagon release, glucagon stimulates release of insulin, and somatostatin inhibits release of both insulin and glucagon. Insulin secretion by $\beta$ cells declines with age. The diminished insulin responsiveness may be partly attributable 
to increased somatostatin. However, the mass of the endocrine pancreas is three- to four-fold greater in the mature ad libitumfed rat than in 2-month-old rats and therefore the total insulin secretion of the pancreas is nearly the same. The greater islet cell mass is due to larger number and volume density of $\beta$ cells while $\alpha$ and $\delta$-cell populations remain approximately the same.

\section{Technical Note}

Generally used preferred terms of systemic nonproliferative lesions across organ systems, such as hemorrhage or thrombosis are included in separate INHAND manuscripts currently under preparation and are therefore not included in this document except where there are distinguishing features of the process relevant to the endocrine system. Similarly, systemic tumors such as lymphoma or histiocytic sarcoma are described in separate documents under the hematopoietic system and therefore are also not discussed in this document. Tumors that occur at many sites or locations within the body are described under the specific organ system considered the most appropriate such as schwannoma in the nervous system, or hemangiosarcoma under the cardiovascular system and which will be described in sections of other INHAND guides.

\section{Pituitary}

\section{A. Nonproliferative Lesions}

\section{Aberrant craniopharyngeal structures (N) Pituitary Gland}

(Figures 1 and 2)

Species

Mouse; Rat.

Pathogenesis/cell of origin

Remnants of oro-pharyngeal epithelium of craniopharyngeal duct (Rathke's pouch).

\section{Diagnostic Features}

- Located in the neurohypophysis or between pars intermedia and pars nervosa.

- Proliferation of acinar, tubular, or fusiform cellular structures.

- Acinar structures are characterized by abundant eosinophilic cytoplasm and round nuclei located on the basal side. Eosinophilic secretory granules are occasionally observed at the side of the lumen. These granules are positive for PAS reaction and negative for Alcian blue, being very similar to the features of the serous salivary gland.

- Tubular structures composed of cuboidal cells with round nuclei located on the basal side. Tubular lumen is narrow.

- Fusiform cells having scant cytoplasm with round or oval nuclei, and one to two cell layers thick.

- In many cases associated with Rathke's cleft cyst(s).

Differential Diagnoses

Cyst(s):

- Cyst(s) lined by cuboidal to pseudostratified columnar (ciliated) epithelium containing proteinaceous material.

- No tubular or acinar structures.

- No infiltration of the pars nervosa.

Persistent Rathke's Pouch:

- Located between pars distalis and pars intermedia.

- Epithelial lining is usually ciliated but can be squamous, cuboidal, or columnar.

Pseudocyst(s):

- Lack of epithelial lining.

- Lined by normal or degenerated pituitary endocrine cells.

Craniopharyngioma, benign:

- May compress pituitary gland or brain stem.

- Neoplastic cells form keratinizing squamous epithelium with papillary and cyst formation, or cord-like proliferations associated with marked hyperkeratosis and parakeratosis.

Metastatic tumors:

- Metastasis of squamous cell carcinoma with a primary 
site in a different organ.

\section{References}

(Botts et al. 1994; Capen 1996a; Faccini et al. 1990; Frith et al. 2000; Iwata et al. 2000; Karbe and Ernst 1996; MacKenzie and Boorman 1990; Mahler and Elwell 1999; Schaetti et al. 1995)

\section{Angiectasis (N) Pituitary Gland (Figure 3)}

Species

Mouse; Rat.

Synonym(s)

Hemangiectasis; Hemangiectasia; Teleangiectasis.

Pathogenesis/cell of origin

Vasculature of pituitary gland parenchyma.

\section{Diagnostic Features}

- Distended, blood-filled vessels lined by well differentiated endothelial cells.

- Usually present in pars distalis.

- May distort the normal architecture of the pituitary gland.

\section{Differential Diagnoses}

Hemangioma:

- Focal proliferation of blood-filled spaces lined with prominent uniform endothelial cells, distorting the architecture of the affected tissue.

Congestion:

- Diffuse dilatation of blood filled vessels not distorting the architecture of the affected tissue.

Hemorrhage:

- Extravascular blood present in the pituitary gland parenchyma. Chronic hemorrhage maybe associated with hemosiderin-laden macrophages.

\section{Comment}

Angiectasis is relatively frequent in the highly vascular pars distalis of aging rats and mice. Angiectasis is most frequently seen as a component of hyperplasia and neoplasia of the pars distalis, but may also occur in the pars distalis without proliferative lesions.

\section{References}

(Attia 1985; Botts et al. 1994; Faccini et al. 1990; Frith et al. 2000; Mahler and Elwell 1999)

\section{Aplasia/Hypoplasia (N) Pituitary Gland}

Species

Mouse; Rat.
Pathogenesis/cell of origin

Endocrine cells of the pars distalis and intermedia of the pituitary gland.

\section{Diagnostic Features}

- Developmental lack of endocrine cells.

- Can affect the entire gland or one or more cell types.

- May be focal, multifocal or diffuse.

Differential Diagnoses

Atrophy:

- Acquired loss of endocrine cells.

Comment

Complete or partial absence of the pituitary gland has been reported in mice.

\section{References}

(Bossé et al. 1997; Greaves 2012c; Kalter 1968; Wallace et al. 1999)

\section{Atrophy (N) Pituitary Gland (Figures 4 and 5)}

Species

Mouse; Rat.

Pathogenesis/cell of origin

Endocrine cells of the pars distalis and intermedia of the pituitary gland.

\section{Diagnostic Features}

- Acquired loss of endocrine cells.

- Can affect the entire gland or one or more cell types.

- May be focal, multifocal or diffuse.

\section{Differential Diagnoses}

Aplasia/Hypoplasia:

- Developmental lack of endocrine cells.

\section{Comment}

Atrophy can be due to lack of hypophyseal stimulation or endocrine negative feedback, and may result in reduced weight of the pituitary due to loss of anterior pituitary cell mass. Diffuse atrophy of a pituitary cell population also occurs in association with hyperplasia of other cell types or focally around the margins of expanding pituitary neoplasms. Loss of pituitary cells may be seen with advancing age in rats, associated with reduction in the hypothalamic content of releasing hormones and the capacity of the pituitary to synthesize or release hormones, such as FSH, LH and TSH. A number of other external factors have been shown to adversely diminish pituitary function (including viral infection and autoimmune damage). Reduction of pituitary cells can also occur following pharmacological suppression. 
References

(Capen 1996a; Chandra et al. 2013; Greaves 2012c)

Cyst (N) Pituitary Gland (Figures 6, 7, 8, and 9)

Species

Mouse; Rat.

Pathogenesis/cell of origin

The origin of a cyst usually cannot be determined from its structure, position, or contents.

Some cysts may be derived from persistent remnants of the craniopharyngeal duct (Rathke's pouch).

Diagnostic Features

- Usually located in pars distalis.

- Single layer of cuboidal to columnar epithelium.

- Epithelial lining may be ciliated and may contain mucous cells.

- Usually contain eosinophilic to amphophilic mucoproteinaceous material.

- Simple or multilocular.

Differential Diagnoses

Pseudocyst(s):

- Lack of epithelial lining.

- Lined by normal or degenerate pituitary endocrine cells.

Persistent Rathke's Pouch:

- Usually located between pars distalis and pars intermedia.

- Presence of tubular or glandular structures.

Craniopharyngeal structures, aberrant:

- Localized in the pars nervosa or between pars intermedia and pars nervosa.

- Tubular structures with narrow lumen.

- Cuboidal cells with round cuboidal nuclei located on the basal pole.

\section{Comment}

Cysts in the adenohypophysis or neurophyophysis are commonly observed as background lesions in laboratory rats.

\section{References}

(Capen 1996a; Chandra et al. 2013; Faccini et al. 1990; Frith et al. 2000; Iwata et al. 2000; MacKenzie and Boorman 1990; Mahler and Elwell 1999; Quintanar-Stephano et al. 2001; Schaetti et al. 1995)

Fibrosis (N) Pituitary Gland (Figures 4 and 5)

\section{Species}

Mouse; Rat.

\section{Pathogenesis/cell of origin}

Collagen deposition by fibroblasts following inflammation, necrosis, or hemorrhage.
Diagnostic Features

- Increased number of fibroblasts and interstitial collagen that may be associated with chronic inflammation.

References

(MacKenzie and Boorman 1990; Robbins and Contran 2010b)

Gliosis, pars nervosa (N) Pituitary Gland (Figure 10)

Species

Mouse; Rat.

Pathogenesis/cell of origin

Glial cells of the pars nervosa.

Diagnostic Features

- Increased numbers of glial cells (specialized astrocytes) in the pars nervosa.

- The glial cells may be polyhedral or spindle-shaped cells.

Differential Diagnoses

Infiltrate, Inflammatory Cell:

- Small numbers of inflammatory cells within the interstitium.

- Usually consists of mononuclear cells but may have a small number of neutrophils.

\section{Comment}

Pituicytes are specialized astrocytes that may be parenchymatous or fibrous type. Immunohistochemical markers include glial fibrillary protein (GFAP) and S100 beta protein. Dehydration has been shown to increase astrocyte proliferation in the pars nervosa of adult rats.

\section{References}

(Alonso et al. 2003; Murugaiyan and Salm 1995; Vazquez et al. 1987; Wei et al. 2009)

\section{Hemorrhage (N) Pituitary Gland (Figure 11)}

Species

Mouse; Rat.

\section{Pathogenesis/cell of origin}

Vasculature of pituitary gland parenchyma; vascular injury or hemorrhagic diathesis.

Diagnostic Features

- Free erythrocytes in the extravascular space.

- Chronic hemorrhage usually is accompanied by hemosiderin-laden macrophages.

- Cholesterol clefts and fibrosis may also occur.

- Hemorrhage can occur into Rathke's cleft.

Differential Diagnoses

Angiectasis:

- Blood present within dilated blood vessels. 


\section{Comment}

Iron stains (such as Prussian blue) may be useful in differentiating hemosiderin at sites of long-standing hemorrhage from other brown pigments, e.g. lipofuscin and melanin.

\section{References}

(Attia 1985; Capen 1996a; Faccini et al. 1990; Frith et al. 2000; MacKenzie and Boorman 1990; Mahler and Elwell 1999)

\section{Hypertrophy, pars intermedia (N) Pituitary Gland}

Species

Mouse; Rat.

\section{Pathogenesis/cell of origin}

Stimulation of growth of the endocrine cells of the pars intermedia.

\section{Diagnostic Features}

- Enlargement of endocrine cells of the pars intermedia.

\section{Differential Diagnoses}

Hyperplasia, pars intermedia:

- Focal increase in the number of endocrine cells is present.

\section{Hypertrophy, pars distalis (N) Pituitary Gland}

(Figures 12, 13)

\section{Species}

Mouse; Rat.

\section{Pathogenesis/cell of origin}

Stimulation of growth of the endocrine cells of the pars distalis.

\section{Diagnostic Features}

- Enlargement of individual endocrine cells of the pars distalis.

- Usually affects a single cell type.

- May be associated with cytoplasmic vacuolation.

- Cytoplasm may have decreased staining intensity, especially basophils or acidophils.

\section{Differential Diagnoses}

Hyperplasia, pars distalis:

- Focal increase in the number of endocrine cells is present.

\section{Comment}

Hypertrophy is one of the more commonly observed responses of the pars distalis in toxicity studies. It is usually not due to a direct effect on the pituitary gland, but is related to toxicity or decreased function of the target endocrine organs or increased metabolism and/or excretion of the target organ hormones. The resulting reduction in circulating endocrine hormone concentrations removes the negative feedback mechanism to the hypothalamic-hypophyseal axis and causes stimulation of specific cell types in the pars distalis. Cells first undergo degranulation followed by hypertrophy. Individual cells may become vacuolated with displaced nuclei. This gives the cells a signet-ring appearance. Vacuolated endocrine cells in the pars distalis of neutered animals are sometimes called 'castration cells'; however, the preferred terminology is hypertrophy and vacuolation. If the condition continues, hyperplasia may occur. The specific cell types affected in the pars distalis can be identified using immunocytochemistry for their respective hormones.

\section{References}

(Attia 1985; Botts et al. 1994; Capen 1996a; Chandra et al. 2013; Faccini et al. 1990; Frith et al. 2000; Greaves 2012c; Mahler and Elwell 1999)

\section{Infiltrate, inflammatory cell (N) Pituitary Gland}

Species

Mouse; Rat.

\section{Pathogenesis/cell of origin}

Accumulation of small number of inflammatory cells; uncertain pathogenesis or local immune response.

\section{Diagnostic Features}

- Small numbers of inflammatory cells within the interstitium.

- Usually consists of mononuclear cells but may have a small number of neutrophils.

- May be focal or multifocal.

\section{Differential Diagnoses}

Inflammation:

- Usually more extensive.

- Typically associated with tissue damage and/or vascular changes.

\section{References}

(Carlton and Gries 1996; Frith et al. 2000; MacKenzie and Boorman 1990; Mahler and Elwell 1999)

\section{Inflammation (N) Pituitary Gland}

Species

Mouse; Rat.

\section{Pathogenesis/cell of origin} Inflammatory change.

\section{Diagnostic Features}

- Inflammatory cells can be neutrophilic, lymphoplasmacytic, histiocytic, or pyogranulomatous.

- Epithelial cell degeneration, necrosis, or loss may be present.

- Typically associated with tissue damage and/or vascular changes. 


\section{Differential Diagnoses}

Infiltrate, inflammatory cell:

- Not associated with tissue damage or vascular changes.

Sarcoma, histiocytic:

- Pituitary gland parenchyma is infiltrated and replaced by neoplastic and pleomorphic histiocytic cells.

Lymphoma, malignant:

- Pituitary gland parenchyma is infiltrated and replaced by neoplastic lymphoid cells.

- Spleen and lymph nodes are frequently involved.

\section{Comment}

Inflammation of the pituitary is rare in rodents. Infiltration by inflammatory cells may be seen as an extension of meningitis, otitis, sinusitis, or septicemia.

\section{References}

(Carlton and Gries 1996; Frith et al. 2000; Greaves 2012c;

MacKenzie and Boorman 1990; Mahler and Elwell 1999)

\section{Osseous metaplasia (N) Pituitary Gland}

Species

Mouse; Rat.

\section{Pathogenesis/cell of origin}

Differentiation of stem cells or undifferentiated mesenchymal cells to osteoblasts. Adaptive response to various degenerative and regenerative processes or in association with a primary tumor or metastasis to the pituitary gland.

\section{Diagnostic Features}

- Presence of mineralized bone matrix (osteoid) lined by osteoblasts.

- Ectopic bone is composed by trabecular bone.

- Fatty or hematopoietic bone marrow may develop in intertrabecular spaces.

- Localized in pars distalis, intermedia or nervosa.

\section{Differential Diagnoses}

Mineralization:

- Inorganic mineral substance.

- Osteoblasts and trabecular bone are absent.

- May be intracellular or extracellular.

- May follow necrosis or hemorrhage.

- Homogeneous or granular bluish to purple material.

\section{References}

(MacKenzie and Boorman 1990; Robbins and Contran 2010a)

\section{Persistent Rathke's pouch (N) Pituitary Gland}

Species

Mouse; Rat.
Pathogenesis/cell of origin

Remnants of oro-pharyngeal epithelium of craniopharyngeal duct (Rathke's pouch).

Diagnostic Features

- Variably-sized tubular or glandular structures.

- Usually located between pars distalis and pars intermedia.

- Epithelial lining is usually ciliated, but can be squamous, cuboidal, or columnar.

- May be associated with dilatation of Rathke's cleft.

\section{Differential Diagnoses}

Cyst(s):

- Cyst(s) are lined by cuboidal to pseudostratified columnar (ciliated) epithelium containing proteinaceous material.

- No tubular or glandular structures.

- Usually in the pars distalis.

Craniopharyngeal structures, Aberrant:

- Tubular structures with a narrow lumen.

- Localized in the pars nervosa or between pars intermedia and pars nervosa.

- Cuboidal cells with round nuclei located on the basal pole.

Pseudocyst(s):

- Lack of epithelial lining.

- Lined by normal or degenerate pituitary endocrine cells.

Craniopharyngioma, benign:

- Neoplastic cells form a keratinizing squamous epithelium.

- Cell proliferation without infiltration outside of the pituitary gland.

Metastatic tumors:

- Metastasis of squamous cell carcinoma with a primary site in a different organ.

\section{Comment}

Aberrant craniopharyngeal structures are described in the rat and in the Syrian hamster. Histological features as well as proposed origin are similar to these described for the persistence of the Rathke's pouch, but they are localized in the neurohypophysis.

\section{References}

(Botts et al. 1994; Capen 1996a; Faccini et al. 1990; Frith et al. 2000; Iwata et al. 2000; Karbe and Ernst 1996; MacKenzie and Boorman 1990; Mahler and Elwell 1999; Schaetti et al. 1995)

Pigment (N) Pituitary Gland (Figures 4 and 5)

Species

Mouse; Rat. 
Modifiers

Hemosiderin; Lipofuscin.

\section{Pathogenesis/cell of origin}

Accumulation of colored products derived from cellular or erythrocyte (hemoglobin) breakdown or lipid peroxidation of cellular membranes.

\section{Diagnostic Features}

Hemosiderin:

- Yellow to brown pigment.

- Coarsely or finely granular intracytoplasmic pigment.

- Usually present in histiocytes or macrophages after hemorrhage or prolonged congestion.

- Can occur in parenchymal cells or folliculostellate cells.

- Positive for iron stains, such as Prussian blue.

Lipofuscin:

- Yellow to brown pigment.

- Finely granular intracytoplasmic, often perinuclear, pigment.

- In parenchymal cells and folliculostellate cells.

- May exhibit autofluorescence under ultraviolet light.

- Usually PAS-positive, acid fast, and sudanophilic.

\section{Differential Diagnoses}

Formalin pigment (Acid hematin):

- Artifact that occurs when tissue rich in blood comes in contact with acid solutions of formalin.

- Brown to black, finely granular pigment.

- Occurs mainly in blood vessels and in tissues where there are large accumulations of red blood cells.

- Negative for iron. Formaldehyde-derived artifacts can be removed by the Kardasewitsch method.

\section{Comment}

Lipofuscin is a pigment that accumulates in post-mitotic, slow-dividing, and aging cells. The accumulation of this pigment can be accelerated by treatment with drugs and chemicals, trauma, circulatory disturbances and dietary abnormalities (including vitamin E deficiency). Lipofuscin can be an indicator of free radical injury and lipid peroxidation.

\section{References}

(Ackermann 2012; MacKenzie and Boorman 1990; Robbins and Contran 2010a)

\section{Pseudocyst (N) Pituitary Gland (Figure 14)}

\section{Species}

Mouse; Rat.

\section{Pathogenesis/cell of origin}

Endocrine cells of the pars distalis.

\section{Diagnostic Features}

- Irregular space between endocrine cells that is empty or contains proteinaceous fluid.

- Usually occurs in pars distalis, but also can be seen in the pars intermedia.

- Lack of epithelial lining.

- Lined by normal or degenerate pituitary endocrine cells.

Differential Diagnoses

Cyst(s):

- Lined by a single layer of cuboidal to columnar epithelium.

- Epithelial lining may be ciliated or contain mucous cells.

- Usually contains eosinophilic to amphophilic mucoproteinaceous material.

Persistent Rathke's Pouch:

- Presence of tubular or glandular structures.

- Usually located between pars distalis and pars intermedia.

\section{References}

(Capen 1996a; Faccini et al. 1990; Frith et al. 2000; MacKenzie and Boorman 1990; Mahler and Elwell 1999)

\section{Thrombus (N) Pituitary Gland}

Species

Mouse; Rat.

\section{Pathogenesis/cell of origin}

Vasculature of pituitary gland parenchyma or major pituitary arteries and veins.

The pathogenesis involves either injury to endothelium, altered blood flow, hypercoagulability or thromboembolism.

\section{Diagnostic Features}

- Intravascular amorphous pink/gray material that can be homogeneous, fibrillar, or laminated and may contain leucocytes and erythrocytes.

- Usually adheres to the blood vessel wall, but may not be visible in the section.

\section{Differential Diagnoses}

Postmortem clot:

- Few or no leucocytes.

- Lamination absent or very fine filaments.

- No attachment to blood vessels.

\section{Comment}

Thrombosis of the major pituitary artery and subsequent infarction of the pituitary are rare in rats and mice. Thrombosis may be associated with mononuclear cell leukemia in rats. Thrombosis may also result from endothelial cell damage following chemical exposure or to drug toxicity. 


\section{References}

(Capen 1996a; Faccini et al. 1990; Frith et al. 2000; MacKenzie and Boorman 1990; Mahler and Elwell 1999)

\section{Vacuolation (N) Pituitary Gland (Figure 15)}

Species

Mouse; Rat.

Pathogenesis/cell of origin

Endocrine cells of the pars distalis.

\section{Diagnostic Features}

- Endocrine cells contain a large central or several cytoplasmic vacuoles that displace the nucleus peripherally.

- Vacuolated cells are usually hypertrophic.

\section{Comment}

Decreased function of the target endocrine organs of the pars distalis or increased metabolism and/or excretion of the target organ hormones cause a decrease in circulating endocrine hormone concentrations. This reduces negative feedback to the hypothalamic-hypophyseal axis and causes specific cells in the pars distalis to increase their production and secretion of target organ stimulating hormones. Cells first undergo degranulation followed by hypertrophy. Individual cells become vacuolated with displaced nuclei. This gives the cells a signet-ring appearance. Vacuolated endocrine cells in the pars distalis of neutered animals are sometimes called 'castration cells'; however, the preferred terminology is hypertrophy and vacuolation. If the condition continues, hyperplasia occurs. The specific cell types affected in the pars distalis can be identified using immunocytochemistry for their respective hormones.

\section{References}

(Capen 1996a; Greaves 2012c; MacKenzie and Boorman 1990; Mahler and Elwell 1999)

\section{B. Proliferative Lesions}

\section{Hyperplasia, pars distalis (N) Pituitary Gland}

(Figures 16 and 17)

Species

Mouse; Rat.

Pathogenesis/cell of origin

Endocrine cells of the pars distalis.

\section{Diagnostic Features}

Focal:

- Focal increase in the number of endocrine cells.

- Usually affects only one cell type.

- Not well demarcated.

- No compression or only minimal compression affecting less than one quadrant.
- Sinusoids within the affected area may be dilated without affecting growth pattern.

- Cells may be enlarged.

- Cells are uniform in shape.

- Cellular pleomorphism or atypia is not present.

- Diameter is usually smaller than $50 \%$ of the width of the pars distalis.

- Cells do not extend into pars nervosa.

Diffuse:

- Diffuse or nodular increase in the number of endocrine cells.

- Cells may appear crowded with increased density.

- Usually affects only one cell type.

- Sinusoids within the affected area may be dilated without affecting growth pattern.

- Affects all or most of the lobes.

Differential Diagnoses

Adenoma, pars distalis:

- Compression in at least one quadrant is present.

- Cells exhibit pleomorphism and atypia.

- Diameter is often larger than $50 \%$ of the width of the pars distalis.

Hypertrophy, pars distalis:

- Cells are increased in size, with no evidence of hyperplasia.

\section{Comment}

In hyperplastic lesions, the cell type of origin cannot be determined with H\&E stain. Specific identification requires immunohistochemistry. Reticulin stains may be useful in differentiating hyperplasia from adenoma. The regular network of reticulin fibers separating cell nests in normal tissue is maintained in hyperplastic foci, but becomes abnormal or dispersed in adenomas. In the mouse, reticulin fibers are scant that their distribution is often not discernible. In the rat, focal hyperplastic lesions of the pars distalis usually are multiple and most frequently consist of chromophobic cells. The size or width of focal hyperplasia is a weak criterion for differentiation from an adenoma due to variability in the plane of sectioning. Diffuse hyperplasia occurs in rats as a background lesion in aged animals, administration of sex hormones, or other factors causing prolonged changes to the endocrine system. Diffuse hyperplasia of luteotrophs is expected during lactation. Since prolactinoma is the most common pituitary adenoma, hyperplastic foci probably are derived from prolactin-producing cells.

\section{References}

(Attia 1985; Botts et al. 1994; Capen 1996a; Capen et al. 2001; Chandra et al. 2013; Faccini et al. 1990; Greaves 2012c; Lee et al. 1982; Liebelt 1994; Mahler and Elwell 1999; McComb et al. 1985; Osamura 1996b, c) 


\section{Hyperplasia, pars intermedia (N) Pituitary Gland}

(Figures 18 and 19)

Species

Mouse; Rat.

Pathogenesis/cell of origin

Endocrine cells of the pars intermedia.

\section{Diagnostic Features}

Focal:

- Focal increase in the number of endocrine cells.

- No compression or only minimal compression affecting less than one quadrant.

- Lobular architecture of pars intermedia is maintained.

- Cell morphology is similar to normal pars intermedia cells, but the cytoplasm is often paler or more eosinophilic.

- Hyperplastic cells can extend into the pars nervosa.

Diffuse:

- Diffuse increase in the number of endocrine cells.

- Hyperplastic cells can extend into the pars nervosa.

\section{Differential Diagnoses}

Hypertrophy, pars intermedia:

- Pars intermedia cells are increased in size with no evidence of hyperplasia.

Adenoma, pars intermedia:

- Lobular architecture is not maintained.

- Compression in more than one quadrant is present.

\section{Comment}

Focal hyperplasia is a precursor of adenoma. Hyperplasia is relatively frequent in rats and rare in mice. Diffuse hyperplasia is more common in old rats. Pars intermedia cells are usually immunohistochemically positive for ACTH and $\alpha$-MSH.

\section{References}

(Botts et al. 1994; Capen 1996a; Capen et al. 2001; Carlton and Gries 1996; Greaves 2012c; Liebelt 1994; Mahler and Elwell 1999)

\section{Adenoma, pars distalis (B) Pituitary Gland}

(Figures 20 and 21)

Species

Mouse; Rat.

Synonym

Adenoma, Pituitary.

Pathogenesis/cell of origin

Endocrine cells of the pars distalis.

\section{Diagnostic Features}

- Usually well demarcated.
- May be highly vascular with dilated blood vessels.

- Adenomas may be cystic or have areas of hemorrhage.

- The cell pattern may be trabecular or solid.

- Cells and nuclei are usually enlarged.

- Cells have a homogenous appearance, but cellular pleomorphism or atypia may be present.

- There is usually compression of the pars distalis in more than one quadrant of the adenoma.

- Adenomas are usually larger than $50 \%$ of the width of the pars distalis.

- Adenomas do not invade other organs, but may have infiltrative growth within the gland including the pars intermedia and nervosa.

- Large adenomas may compress the brain.

\section{Differential Diagnoses}

Hyperplasia, pars distalis (focal):

- No compression or minimal compression of the pars distalis.

- Cells do not exhibit pleomorphism or atypia.

- Diameter is usually less than $50 \%$ of the width of the pars distalis.

Adenoma, pars intermedia:

- Whorl formation and lobular pattern may be present.

- Neoplastic cells exhibit similarity to pars intermedia cells.

Carcinoma, pars distalis:

- Invasion through the meninges into other organs (brain or sphenoid bone) is present.

\section{Comment}

Evidence suggests that focal hyperplasia is a precursor of pituitary adenoma. Size of the lesion is considered a weak criterion for differentiation of focal hyperplasia from adenoma due to variability in the plane of sectioning. The classic differentiation of adenomas into chromophobic, basophilic and acidophilic has lost its importance since immunohistochemical techniques permit the specific recognition of cell types in the majority of cases. For routine adenoma documentation in standard 2-year carcinogenicity bioassays, additional immunohistochemical characteriziation is usually not needed. In rats, prolactinomas are the most common pituitary adenomas. Adenomas that produce other hormones of the pars distalis have been identified, sometimes in combination. Moreover, one tumor cell may occasionally produce more than one hormone, e.g., prolactin and growth hormone. Prolactinomas are associated with hyperprolactinemia. Prolactinomas can be induced in rats by sustained stimulation with estrogens. Null-cell adenomas contain no recognizable hormonal antigen, but may produce the common $\alpha$-subunit of gonadotropins and thyroid stimulating hormone. The high incidence of pituitary adenomas in aged rats is reduced by dietary restriction.

In mice, spontaneous pars distalis neoplasms have been 
less well characterized by immunocytochemistry than those found in rats or humans. Compression is often absent even in large adenomas.

\section{References}

(Botts et al. 1994; Capen 1996a; Capen et al. 2001; Carlton and Gries 1996; Faccini et al. 1990; Greaves 2012c; Jameson et al. 1992; Kaspareit and Rittinghausen 1999; Liebelt 1994; Lloyd 1990; Mahler and Elwell 1999; Osamura 1996c; Roe et al. 1995; Son 2004; Yasuno et al. 2013)

\section{Adenoma, pars intermedia (B) Pituitary Gland}

(Figures 22, 23, and 24)

\author{
Species \\ Mouse; Rat. \\ Synonym \\ Adenoma, Pituitary.

\section{Pathogenesis/cell of origin} \\ Endocrine cells of the pars intermedia.
}

\section{Diagnostic Features}

- Compression may be present.

- Pattern of the cells may be solid, lobular or pseudofollicular.

- Whorl formation may be present.

- Cells are uniform or pleomorphic.

- Cell morphology is similar to normal pars intermedia cells, but the cytoplasm is often paler or more eosinophilic.

- Often extends into adjacent pars nervosa or pars distalis or may extend into and compress adjacent brain.

- Does not infiltrate organs outside the pituitary.

\section{Differential Diagnoses}

Hyperplasia, pars intermedia (focal):

- Lobular architecture is maintained.

- Compression is not present.

Hyperplasia, pars intermedia (diffuse):

- Not focal, affects the entire pars intermedia.

Adenoma, pars distalis:

- Lobular pattern or whorl formation is not present.

- Cells are not similar to pars intermedia cells.

Carcinoma, pars intermedia:

- Invasion of the brain or sphenoid bone is present.

- No report of this tumor has been made in rats.

\section{Comment}

Adenoma of the pars intermedia in the rat and mouse is much less common than in the pars distalis. Cells resemble those in the normal pars intermedia. Infiltrative tumor cells within the adjacent pars nervosa are usually not a sign of malignancy. Positive immunohistochemical staining for adrenocorticotrophic hormone (ACTH) or $\alpha$-melanocyte stimulating hormone $(\alpha-\mathrm{MSH})$ can be used to confirm the diagnosis of pars intermedia adenoma.

\section{References}

(Attia 1985; Botts et al. 1994; Capen 1996a; Capen et al. 2001; Carlton and Gries 1996; Liebelt 1994; MacKenzie and Boorman 1990; Mahler and Elwell 1999; McComb et al. 1985; Oishi et al. 1992; Osamura 1996a; Rehm et al. 1985)

\section{Ganglioneuroma, benign (B) Pituitary Gland}

(Figures 25 and 26)

Species

Mouse; Rat.

Synonym $(s)$

Gangliocytoma; Ganglion cell tumor; Ganglioglioma.

Pathogenesis/cell of origin

Residual nerve cells in the hypophysis.

Diagnostic Features

- Consists of large, well differentiated ganglion cells and neurofibrils. Rarely the tumor can be composed of immature neuronal cells.

- Compression of the adjacent pituitary tissue.

Differential Diagnoses

Adenoma, pars distalis or pars intermedia:

- Cells have a homogeneous appearance.

- Large ganglion cells are not present.

Pituicytoma:

- Originates in the pars nervosa.

- Sheets of closely packed spindle cells (pituicytes).

Craniopharyngioma, benign:

- Anastomosing cords of keratinizing squamous epithelial cells and cystic spaces lined by squamous epithelium.

\section{Comment}

Ganglioneuromas are very rare tumors in the rat and mouse pituitary. Ganglion cells stain positively with antibodies to neurofilament protein (NF), chromogranin A, synaptophysin or neuron-specific enolase (NSE).

References

(Heath 1996d; Okazaki et al. 1997; Pace and Perentes 2001; Yasui et al. 2009)

\section{Pituicytoma, benign (B) Pituitary Gland}

(Figures 27, 28, and 29)

Species

Mouse; Rat. 
Pathogenesis/cell of origin

Pituicytes (glial cells of neurohypophysis).

Diagnostic Features

- Originates in neurohypophysis.

- Usually well demarcated.

- Compression of adjacent pituitary or brain may be present.

- Sheets of small spindle cells are closely packed.

- Cell borders are indistinct.

- Cytoplasm is eosinophilic and foamy to vacuolated.

- Cytoplasmic granules positive for phosphotungstic acid hematoxylin (PTAH) stain may be present.

- Nuclei are elongated and irregular.

- Nuclear palisading is occasionally present.

- No evidence of cellular pleomorphism.

- May extend into the adenohypophysis.

\section{Differential Diagnoses}

Pituicytoma, malignant:

- Invasion of adjacent pituitary, brain, and sphenoid bone.

Meningioma, benign:

- Does not originate in the pars nervosa.

- Occurs as a meningeal thickening or mass on the surface of the brain or in the ventricular cavities.

Astrocytoma, malignant, low grade or Oligodendroglioma, malignant, low grade or Glioma, mixed, malignant, low grade:

- Originates in the brain.

- Confined to one area of the brain.

\section{Comment}

Positive immunohistochemical staining for S-100 has been described in human pituicytomas and GFAP for rat, mouse and human pituicytomas. The pituicytoma is extremely rare in the rat and mouse. Only one case has been reported in the mouse.

\section{References}

(Botts et al. 1994; Brandão et al. 2010; Capen 1996a; Capen et al. 2001; Carlton and Gries 1996; Figarella-Branger et al. 2002; Fitzgerald et al. 1974; Louis et al. 2007; Satoh et al. 2000; Tekeli et al. 1997; Theuring et al. 1990)

\section{Pituicytoma, malignant (M) Pituitary Gland}

Species

Rat.

\section{Pathogenesis/cell of origin}

Pituicytes (glial cells of neurohypophysis).

\section{Diagnostic Features}

- Originates in neurohypophysis.

- Whirling or irregular growth pattern.

- Compression of the adjacent pituitary and brain may be present.

- Invasion of adjacent pituitary, brain, or sphenoid bone.

- Sheets of small pleiomorphic spindle cells with abundant eosinophilic cytoplasm.

- Cell borders are indistinct.

- Cytoplasmic hyaline granules positive for phosphotungstic acid hematoxylin (PTAH) stain may be present.

- Nuclei are elongated or pleiomorphic.

- Bizarre multinucleate giant cells may be present.

Differential Diagnoses

Pituicytoma, benign:

- No evidence of tissue invasion.

- Minimal cellular and nuclear pleiomorphism.

Glioblastoma, giant cell type:

- Originates in the brain or pituitary gland.

- Confined to one area of the brain.

\section{Comment}

The tumor cells stain immunohistochemically strongly positive for vimentin and glial fibrillary acidic protein (GFAP) and weakly for ED-1 and S-100 protein. The malignant pituicytoma is extremely rare. One case has been reported in a Sprague-Dawley rat.

\section{References}

(Moroki et al. 2015)

\section{Carcinoma, pars distalis (M) Pituitary Gland}

(Figures 30 and 31)

Species

Mouse; Rat.

Synonym

Carcinoma, Pituitary.

Pathogenesis/cell of origin

Endocrine cells of the pars distalis.

\section{Diagnostic Features}

- May be highly vascular or angiomatous.

- May be cystic or have regions of hemorrhage.

- Architecture may be trabecular or solid.

- Cytomegaly and karyomegaly.

- Cells may exhibit pleomorphism and atypia.

- Invasion through the meninges into the brain or sphenoid bone.

\section{Differential Diagnoses}

Adenoma, pars distalis:

- Invasion into other organs is not present, but infiltrative growth within the gland, including the pars nervosa may be present. 
Adenoma, pars intermedia:

- Whorl formation and lobular pattern may be present.

- Neoplastic cells exhibit similarity to pars intermedia cells.

- Positive immunohistochemical staining for ACTH (or $\alpha-\mathrm{MSH}$ ) can be used to confirm the diagnosis of adenoma, pars intermedia.

Carcinoma, pars intermedia:

- Neoplastic cells exhibit similarity to pars intermedia cells.

- Positive immunohistochemical staining for ACTH (or $\alpha-\mathrm{MSH})$ can be used to confirm the diagnosis of carcinoma pars intermedia.

\section{Comment}

The ability to diagnose carcinoma is reduced if adjacent brain and sphenoid bone are not evaluated histopathologically. Reactive meningeal cells surrounding the tumor are not a sign of malignancy. Metastases of spontaneous pituitary carcinomas usually do not occur in distant organs. Infiltration of other pituitary regions and compression of the brain are not considered features of malignancy because these also occur with locally expansile adenomas.

\section{References}

(Botts et al. 1994; Capen 1996a; Capen et al. 2001; Carlton and Gries 1996; Faccini et al. 1990; Greaves 2012c; Hosokawa et al. 1993; Liebelt 1994; Mahler and Elwell 1999)

\section{Carcinoma, pars intermedia (M) Pituitary Gland}

\section{Species}

Mouse.

\section{Synonym}

Carcinoma, Pituitary.

Pathogenesis/cell of origin

Endocrine cells of the pars intermedia.

\section{Diagnostic Features}

- Compression may be present.

- Lobular architecture is not maintained.

- Cells are uniform or pleomorphic.

- Cell morphology is similar to normal pars intermedia cells, but the cytoplasm is often paler or more eosinophilic.

- Invasion outside the pituitary gland into the meninges, brain or sphenoid bone.

\section{Differential Diagnoses}

Adenoma, pars intermedia:

- Invasion of the brain or other structures is not present but infiltrative growth within the gland including the pars nervosa may be present.
Adenoma, pars distalis:

- Invasion of the brain or other structures is not present.

- Cells do not have similarity to pars intermedia cells.

Carcinoma, pars distalis:

- Cells do not have similarity to pars intermedia cells.

\section{Comment}

Carcinoma, pars intermedia, has not been reported in rats. Reports of carcinomas arising from the pars intermedia of the mouse pituitary gland are very rare. Reactive meningeal cells surrounding the tumor are not a sign of malignancy. Positive immunohistochemical staining for ACTH or $\alpha$-MSH can be used to confirm the diagnosis of pars intermedia carcinoma.

\section{References}

(Botts et al. 1994; Capen et al. 2001; Liebelt 1994; Mahler and Elwell 1999; Yasui et al. 2008)

Craniopharyngioma, benign (B) Pituitary Gland (Figure 32)

Species

Mouse; Rat.

\section{Pathogenesis/cell of origin}

Remnants of oropharyngeal epithelium of the craniopharyngeal duct (Rathke's pouch).

\section{Diagnostic Features}

- Develops within or in close proximity to the pituitary gland.

- Compression of the pituitary gland or brain.

- Forms well differentiated squamous epithelium with papillary tumor growth and cyst formation, or cord-like neoplastic cells with marked hyperkeratosis and/or parakeratosis.

- Expansile neoplastic cells and reactive fibrosis may mimic invasion.

\section{Differential Diagnoses}

Cyst:

- Cysts are lined by cuboidal to pseudostratified columnar (ciliated) epithelium and contain proteinaceous material.

Craniopharyngioma, malignant:

- Invasion outside the pituitary gland.

Craniopharyngeal structures, aberrant:

- Glandular structures with no keratinization

Metastatic tumors:

- Metastasis of squamous cell carcinoma from a distant primary site.

\section{Comment}

Only one craniopharyngioma in mice has been reported, which was diagnosed to be malignant. A genetically modified mouse model of benign craniopharyngioma has been 
developed (Apps and Martinez-Barbera, 2017). The morphology of the induced tumors resembled the features for ameloblastomas of the teeth.

\section{References}

(Anderson and Capen 1978; Apps and Martinez-Barbera 2017; Botts et al. 1994; Capen 1996a; Capen et al. 2001; Carlton and Gries 1996; Fitzgerald et al. 1971; Heider 1986)

\section{Craniopharyngioma, malignant (M) Pituitary Gland}

\section{Species}

Mouse; Rat.

\section{Pathogenesis/cell of origin}

Remnants of oropharyngeal epithelium of craniopharyngeal duct (Rathke's pouch).

\section{Diagnostic Features}

- Develops within or in close proximity to the pituitary gland.

- Compression of the pituitary gland or brain.

- Forms well differentiated squamous epithelium with papillary tumor growth and cyst formation, or cord-like neoplastic cells associated with marked hyperkeratosis and/or parakeratosis.

- Invasion of the brain or sphenoid bone.

\section{Differential Diagnoses}

Craniopharyngioma, benign:

- No invasion outside the pituitary gland.

Metastatic tumors:

- Metastasis of squamous cell carcinoma from a distant primary site.

\section{Comment}

In the mouse, only one case is reported that had invasive growth into cerebral structures and was classified as malignant. Craniopharyngioma, malignant is extremely rare in rats. Case reports of craniopharyngiomas in rats often avoid discussion about malignancy; however, most were probably benign based on the above criteria.

\section{References}

(Anderson and Capen 1978; Botts et al. 1994; Capen 1996a; Capen et al. 2001; Carlton and Gries 1996; Fitzgerald et al. 1971; Heider 1986; Pace et al. 1997)

\section{PineAL GLAND}

\section{A. Nonproliferative Lesions}

Fibrosis (N) Pineal Gland (Figure 33)

Species

Mouse; Rat.

\section{Pathogenesis/cell of origin}

Collagen deposition by fibroblasts following inflammation, necrosis, or hemorrhage.

\section{Diagnostic Features}

- Located within the interstitium at the periphery of the gland and near the pineal stalk, sometimes extending into the gland.

- Increased number of fibroblasts and interstitial collagen.

- May be associated with an inflammatory cell infiltrate.

- Small blood vessels are often present within the fibrosis, and the vessel walls may be hyalinized.

- Collagen fibers can be stained with Masson's trichrome stains.

\section{Comment}

The incidence of fibrosis increases with age.

References

(Tomonari et al. 2012)

Infiltrate, inflammatory cell (N) Pineal Gland (Figure 34)

Species

Mouse; Rat.

\section{Pathogenesis/cell of origin}

Accumulation of small number of inflammatory cells; uncertain pathogenesis or due to local immune response.

\section{Diagnostic Features}

- Occurs in the pia mater or parenchyma of pineal glands.

- Inflammatory cell infiltrate is usually mononuclear cells (lymphocytes and histiocytes).

- Not associated with tissue damage of vascular changes.

\section{Differential Diagnoses}

Sarcoma, histiocytic:

- Pineal gland parenchyma is infiltrated and replaced by neoplastic and pleomorphic histiocytic cells.

\section{Lymphoma:}

- Pineal gland parenchyma is infiltrated and replaced by neoplastic lymphoid cells.

- Spleen and lymph nodes are frequently involved.

\section{Comment}

A low number of mononuclear cells may be seen in male and female rats. In most rats, lymphoid cells are in con- 
nective tissue septae and the adjacent parenchyma. Occasionally, aggregates of lymphoid cells are present in the interior of the gland or the pineal capsule.

\section{References}

(Calvo and Boya 1984; Tomonari et al. 2012)

\section{Mineralization (N) Pineal Gland (Figure 35)}

Species

Mouse; Rat.

\section{Pathogenesis/cell of origin}

Degenerative change of pinealocytes, interstitium, or capsule.

\section{Diagnostic Features}

Intracellular type:

- Irregular basophilic clumps of mineral are present in vacuoles that may represent lipid droplets.

- Cells with mineral may degenerate and mineral will then be present in the extracellular space.

Extracellular type:

- Amorphous spherical concentric layers of mineral are present in the interstitium or capsule.

\section{Comment}

Mineralization has been seen in the pineal glands of $90 \%$ of 17-week-old male Sprague-Dawley rats. Mineralization of the pineal gland in young rats is uncommon based on the authors' experiences. Mineralization of the meninges covering the pineal gland has been reported to increase with age in rats. Small calcareous concretions were observed using electron microscopy in the pineal glands of all aged rats. Most were found in the subcapsular region; however, some were also located in the parenchyma. Mineralization apparently does not interfere with function of the pineal gland.

\section{References}

(Allen et al. 1982; Humbert and Pévet 1995)

Striated muscle fibers (N) Pineal Gland (Figures 36 and 37)

\section{Species}

Mouse; Rat.

\section{Pathogenesis/cell of origin}

Stroma of pineal gland.

\section{Diagnostic Features}

- Skeletal muscle fibers have typical cross-striations.

- Located within the connective tissue.

- Striated muscle fibers may occur with fibrosis.

- Phosphotungstic acid-hematoxylin (PTAH) stain or immunohistochemistry (e.g., desmin) for striated muscle fibers can be used for confirmation.

\section{Comment}

Striated muscle fibers are considered to be an incidental finding in rat pineal glands. Striated muscle fibers were reported to be a rare or common (31 of 96 rats) finding in rats, and reporting of the finding is optional, especially if not related to administration of a chemical. The muscle fibers were most frequently present in the stalk region. Striated muscle was usually located in the wide connective tissue septae near the capsule and consisted of thin myocytes forming bundles of a few muscle fibers.

\section{References}

(Allen et al. 1982; Calvo and Boya 1984; Diehl 1978; Dill 1963; Kristić 1972; Prosenc and Cervós-Navarro 1994; Tomonari et al. 2012)

Vacuolation (N) Pineal Gland (Figure 38)

Species

Mouse; Rat.

Pathogenesis/cell of origin

Degenerative change of pinealocytes.

Diagnostic Features

- Single vacuoles within pinealocytes.

- Scattered throughout the gland.

- Large vacuoles compress nuclei to the cytoplasmic membrane.

- Vacuoles may be clear or occasionally contain flocculent material.

- Size of vacuoles may exceed 4 to 5 times the diameter of normal pinealocytes.

\section{Comment}

Vacuoles are reported to occur with a low incidence in male and female rats and are age-related.

References

(Tomonari et al. 2012)

\section{B. Proliferative Lesions}

\section{Pinealoma, benign (B) Pineal Gland}

(Figures 39, 40, 41, 42, and 43)

Species

Mouse; Rat.

Synonym(s)

Pineocytoma; Benign tumor of pineal gland; Pineal parenchymal tumor (PPT).

Pathogenesis/cell of origin

Differentiated pinealocytes. 
Diagnostic Features

- Midline location at the dorsal surface of the third ventricle.

- Well demarcated.

- The entire pineal gland may be replaced by the neoplasm.

- Well differentiated.

- Cell clusters are divided by a delicate connective tissue.

- Lobulated pattern is present.

- Pseudorosettes may occur.

- Cells usually have a vascular orientation.

- Morphological features of pinealocytes are retained.

- Low nuclear to cytoplasmic ratio is present.

- Tumor cells have dark-staining nuclei with coarse chromatin.

- Some mitotic activity may be present.

\section{Differential Diagnoses}

Pinealoma, malignant:

- Invasive growth.

- May have cytological features of malignancy.

\section{Teratoma, benign:}

- Tumor composed of tissues representing the three germ layers.

\section{Comment}

The description is based only on pinealomas in rats, since only one mouse pineal gland tumor has been reported. The presence of smooth muscle fibers has been reported in a pineal tumor occurring in a Wistar rat. A pineal tumor in a F344 rat contained two types of cells: large, pale-staining and small and dark-staining cells. Tumor cells may be positive for synaptophysin, neuron specific enolase (NSE), or neurofilament markers. Small groups of normal ectopic pinealocytes attached to meninges are rarely reported and should not be interpreted as neoplastic cells.

\section{References}

(Al Zubaidy and Malinowski 1984; Botts et al. 1994; Capen et al. 2001; Coca et al. 1992; Fraser 1986; Furukawa et al. 1999; Götz et al. 1992; Heath and Winokur 1998; Hirato and Nakazato 2001; Jouvet et al. 2006; Koestner and Solleveld 1996; Korf et al. 1990; Krinke et al. 1985; Krinke et al. 2000; Maekawa et al. 1984; Rubinstein 1972; Schachner et al. 1984; Treumann et al. 2015; Vogel and Fuller 2003; Walker et al. 1994; Yamamoto et al. 1991)

\section{Pinealoma, malignant (M) Pineal Gland}

(Figures 44, 45, and 46)

$$
\begin{aligned}
& \text { Species } \\
& \text { Mouse; Rat. }
\end{aligned}
$$

\section{Synonym(s)}

Pineoblastoma; Malignant tumor of pineal gland; Primitive neuroectodermal tumor of central nervous system

(cPNET).

\section{Pathogenesis/cell of origin \\ Differentiated or primordial pinealocytes.}

\section{Diagnostic Features}

- Midline location at the dorsal surface of the third ventricle.

- Invasive growth in adjacent brain parenchyma or ventricles.

- Tumor cells are densely and diffusely arranged.

- Lobulated pattern is usually not apparent.

- Pseudorosettes may occur.

- High nuclear to cytoplasmic ratio is present.

- Tumor cells are pleomorphic with irregular hyperchromatic nuclei and scant cytoplasm.

- High mitotic index.

- Necrotic areas may be common.

- Resembles primitive neuroectodermal tumors (PNET).

\section{Differential Diagnoses}

Pinealoma, benign:

- Features of malignancy including necrosis, high mitotic activity, pleomorphism, and invasive growth are absent.

Medulloblastoma:

- The site of origin is the folia of the cerebellar cortex.

\section{Comment}

A pineal gland tumor has been reported in only one mouse. Midline brain tumors in MSV-SV 40-transgenic mice were shown to originate from the pineal gland (Theuring et al. 1990). Metastases of pineal tumors have not been reported in rodents, but have been described in humans. Tumor cells may be positive for synaptophysin, neuron specific enolase (NSE), or neurofilament markers.

\section{References}

(Botts et al. 1994; Capen et al. 2001; Coca et al. 1992; Fraser 1986; Furukawa et al. 1999; Götz et al. 1992; Heath and Winokur 1998; Hirato and Nakazato 2001; Koestner and Solleveld 1996; Korf et al. 1990; Krinke et al. 1985; Krinke et al. 2000; Kuchelmeister et al. 1994; Rubinstein 1972; Schachner et al. 1984; Theuring et al. 1990; Vogel and Fuller 2003; Walker et al. 1994; Yamamoto et al. 1991) 


\section{ThYROID GLAND}

\section{A. Nonproliferative Lesions}

\author{
Amyloid (N) Thyroid Gland (Figure 47) \\ Species \\ Mouse; Rat. \\ Synonym(s) \\ Amyloidosis; Amyloid deposition.
}

\section{Pathogenesis/cell of origin}

Degenerative change characterized by extracellular deposition of polypeptides derived from immunoglobulin or serum proteins.

\section{Diagnostic Features}

- Diffuse interstitial (extracellular) amorphous pale eosinophilic material.

- Green birefringence using polarized light with positive Congo red stain. Positive with Thioflavine $\mathrm{T}$ stains or specific immunohistochemical antibodies.

\section{Comment}

Spontaneous systemic amyloidosis is common in mice, especially CD-1 and C57BL/6 strains. Amyloidosis can also be induced by administration of Freund's adjuvant and casein. Endocrine amyloid is occasionally present in C-cell neoplasms in rats.

\section{References}

(Chandra et al. 2013; Frith and Chandra 1991; Venalis et al. 2005)

\section{Atrophy (N) Thyroid Gland}

Species

Mouse; Rat.

Pathogenesis/cell of origin

Follicular cells of the thyroid gland.

\section{Diagnostic Features}

- Decreased size of follicular cells, which may be low cuboidal or flattened.

- Acquired loss of follicular cells.

- Thyroid follicles are small with decreased colloid.

- Usually diffuse.

- May be seen with chronic inflammation.

\section{Differential Diagnoses}

Aplasia/Hypoplasia:

- Developmental lack of follicular cells.

Dilatation, Follicular, Diffuse:

- Dilated follicles filled with colloid and lined by low cuboidal or flattened follicular epithelium.

\section{Comment}

Atrophy can be due to lack of thyroid stimulating hormone (TSH) from the pituitary gland or thyrotropic releasing hormone (TRH) from the hypothalamus. Chronic thyroiditis can lead to loss of thyroid follicular cells and subsequent atrophy. Recovery from hypothyroidism (such as iodine deficiency) can lead to diffuse follicular dilatation, which is sometimes called 'colloid' atrophy or involution.

References

(Rosol et al. 2013)

\section{Colloid alteration (N) Thyroid Gland (Figure 48)}

Species

Rat.

Synonym(s)

Colloidal alteration; Altered colloid; Basophilic deposits.

Pathogenesis/cell of origin

Degeneration of colloid in thyroid follicles.

Diagnostic Features

- Stippled, granular or clumped colloid.

- Variable staining characteristics.

- Often mineralized material within the colloid, may contain desquamated follicular cells.

\section{Comment}

Colloid alteration can be observed in the absence of other related morphological changes, but is often associated with thyroid stimulation and is likely related to the normal rapid turnover of thyroid colloid in the rat. It occurs spontaneously and gradually increases with age but is accelerated by diffuse thyroid hypertrophy and hyperplasia. Even if the hypertrophy or hyperplasia resolves, the altered colloid remains. Colloid alteration is usually not reported as a separate diagnosis unless pronounced or treatment-related.

\section{References}

(Capen 1997; Price et al. 1988; Rao-Rupanagudi et al. 1992; Ward and Reznik-Schüller 1980)

\section{Cystic follicle (N) Thyroid Gland (Figure 49)}

Species

Mouse; Rat.

Pathogenesis/cell of origin

Degenerative change.

Diagnostic Features

- Normal follicles that are many times larger than normal and usually focal.

- Usually displaces and compresses adjacent thyroid follicles.

- Lined by a single layer of flattened follicular epithelium. 
- Epithelium does not contain papillary structures.

- Colloid is normal staining or pale.

- Epithelium may contain partial septa from incompletely fused follicles.

\section{Differential Diagnoses}

Dilatation, follicular, diffuse:

- Most follicles are enlarged.

- No compression or displacement of the adjacent follicles.

Hyperplasia, follicular cell, focal, cystic:

- Follicular cells may form papillary projections or microfollicular structures in a segment of the cyst.

- Follicular cells are cuboidal or low columnar but some piling up may be present.

- Cytoplasm may be more eosinophilic or basophilic.

\section{Comment}

Follicles tend to be larger in older mice and this is considered a normal physiologic change. In addition, peripheral follicles in the thyroid gland are normally larger than central follicles and should be distinguished from cystic follicles or follicular dilatation.

\section{References}

(Frith et al. 2000; Hardisty and Boorman 1990; Hardisty and Boorman 1999)

\section{Ectopic tissue, thymus (N) Thyroid Gland (Figure 55)}

\section{Species}

Mouse; Rat.

\section{Pathogenesis/cell of origin}

Congenital foci of ectopic thymus.

\section{Diagnostic Features}

- Thymic tissue located adjacent to or inside the thyroid gland, usually near the hilus of the gland.

- Consists mostly of thymic lymphocytes.

- May contain pale staining clusters of epithelial cells and/ or Hassall's corpuscles.

- Well demarcated.

\section{Differential Diagnoses}

Infiltrate, Inflammatory Cell (Lymphoid):

- Infiltration of lymphocytes without epithelial cells.

- Poorly demarcated.

Lymphoma, Malignant, Systemic Infiltration:

- Consists of malignant lymphocytes.

- Usually not restricted to the thyroid gland.

- Other organs affected.

\section{Comment}

Due to the close association of the thyroid and thymus during embryological development, ectopic thymic tissue may be found in the thyroid or parathyroid gland.

References

(Faccini et al. 1990; Frith et al. 2000; Hardisty and Boorman 1990; Hardisty and Boorman 1999; Parker and Valerio 1996c)

Ectopic tissue, thyroid (N) Thyroid Gland (Figure 57)

Species

Mouse; Rat.

Pathogenesis/cell of origin

Congenital.

Diagnostic Features

- Small aggregates of thyroid follicles near the midline of the body in the neck and mediastinum, in particular near the thymus and aorta.

- Ectopic follicles are identical to normal thyroid, but do not contain $\mathrm{C}$ cells.

\section{Differential Diagnosis}

Persistent Thyroglossal Duct:

- Small cystic or duct-like structures lined by follicles or simple cuboidal to columnar epithelium that may be ciliated.

- Often filled by mucinous material.

- Usually located along the ventral aspect of the larynx on the midline or in the thyroid gland.

\section{Comment}

Ectopic thyroid tissue responds to the same hormonal stimuli (such as thyroid stimulating hormone) as normal thyroidal tissue.

\section{References}

(Chandra et al. 2013; Faccini et al. 1990; Frith et al. 2000; Frith and Fetters 1996b; Hardisty and Boorman 1990; Hardisty and Boorman 1999; Parker and Valerio 1996b)

Hypertrophy, follicular cell (N) Thyroid Gland (Figure 50)

Species

Mouse; Rat.

\section{Pathogenesis/cell of origin}

Stimulation of growth.

\section{Diagnostic Features}

- Follicular cells increased in size and height.

- Generally diffuse distribution.

- Follicular lumen may be decreased and filled with normal staining to pale colloid, which is sometimes mineralized.

- Epithelium does not contain papillary structures. 


\section{Differential Diagnosis}

Hyperplasia, Follicular Cell, Diffuse:

- Increased number of follicular cells.

- Small papillary projections of follicular cells occasionally extend into the lumen.

- Limited piling up of cells.

- Follicular cells may be hyperchromatic without cellular atypia.

\section{Comment}

Male rats normally have more active follicles than females. Follicular cell hypertrophy is a common change seen in toxicity studies in rats induced with a variety of chemicals, such as hepatic microsomal enzymes inducers, which is often associated with hepatocyte hypertrophy. Follicular cell hypertrophy is expected to return to normal if the stimulus is withdrawn. Prolonged follicular cell hypertrophy can progress to hyperplasia and they often occur together. In these cases terminology could include hyperplasia alone, a combined term of hypertrophy/hyperplasia, or record both diagnoses.

Follicular cell hypertrophy is rare in mice.

\section{References}

(Botts et al. 1994; Capen 1996b; Capen et al. 2001; Frith et al. 2000; Greaves 2012d; Maronpot et al. 2010; Yoshizawa et al. 2010; Zabka et al. 2011)

Dilatation, follicular, diffuse (N) Thyroid Gland (Figure 51)

Species

Mouse; Rat.

Synonyms

Follicular dilatation; Colloid goiter; Increased colloid.

Pathogenesis/cell of origin

Accumulation of colloid.

Diagnostic Features

- Most follicles are enlarged and filled with eosinophilic colloid.

- Follicular epithelial cells are low cuboidal to flattened.

\section{Differential Diagnosis}

Follicle, Cystic:

- Many times larger than a normal follicle.

- Usually displaces and compresses the surrounding follicles.

\section{Comment}

Diffuse follicular dilatation with inactive follicular epithelial cells is indicative of inactive thyroid glands in euthyroid animals. This change may occur in animals that have recovered from a state of dietary or chemical-induced hypothyroidism. For example, this occurs in animals that had an iodide deficiency and then became iodide replete.
This change can also be observed when animals have an excess of iodide.

\section{References}

(Frith et al. 2000; Hardisty and Boorman 1999; Kanno et al. 1994; Kanno et al. 1992; Todd 1986)

Infiltrate, inflammatory cell (N) Thyroid Gland (Figure 52)

Species

Mouse; Rat.

Pathogenesis/cell of origin

Accumulation of small number of inflammatory cells; uncertain pathogenesis or local immune response.

Diagnostic Features

- Small numbers of inflammatory cells within the interstitium.

- Usually consists of mononuclear cells but may have a small number of neutrophils.

- May be focal or multifocal.

Differential Diagnosis

Inflammation:

- Usually more extensive.

- Typically associated with tissue damage and/or vascular changes.

References

(Chamanza et al. 2010; Frith et al. 2000)

Inflammation (N) Thyroid Gland (Figure 53)

Species

Mouse; Rat.

Synonyms

Thyroiditis.

Pathogenesis/cell of origin

Inflammatory change.

Diagnostic Features

- Multifocal to diffuse infiltration of lymphocytes, plasma cells, and macrophages. Cellular infiltration is variable but may become diffuse with secondary lymphoid follicle formation. Neutrophilic inflammation is rare.

- Thyroid follicles may be reduced, compressed and smaller with colloid depletion.

- Follicular cells may be hyperplastic in some follicles.

- Typically associated with tissue damage and/or vascular changes.

Differential Diagnosis

Infiltrate, Inflammatory Cell:

- Usually small number of inflammatory cells.

- No tissue damage. 


\section{Comment}

Conventional rat strains rarely develop spontaneous thyroiditis. Inflammatory lesions in the thyroid may arise secondary to systemic disease or regional extension from adjacent tissues, as in the case of polyarteritis nodosa in rats. Spontaneous lymphocytic thyroiditis is described in Buffalo and BioBreeding/Worcester $(\mathrm{BB} / \mathrm{W})$ rat strains with advanced age. Thyroiditis can be also drug induced and the morphologic appearance is similar. An autoimmune process is likely to be the cause as demonstrated by the presence of autoantibodies to thyroglobulin or other thyroid antigens as occurs with Hashimoto's Disease in humans.

\section{References}

(Chandra et al. 2013; Frith et al. 2000; Greaves 2012d; Sandusky and Todd 1996)

\section{Mineralization (N) Thyroid Gland (Figure 54)}

\section{Species}

Mouse; Rat.

\section{Synonyms}

Calcification.

\section{Pathogenesis/cell of origin}

Degenerative change.

\section{Diagnostic Features}

- Irregular basophilic clumps of mineral within the colloid of thyroid follicles.

- Some affected follicles may contain pale colloid.

\section{Comment}

Aging change, may be increased in stimulated thyroid follicles.

Common in rats.

\section{References}

(Frith et al. 2000; Hardisty and Boorman 1990)

\section{Persistent thyroglossal duct (N) Thyroid Gland (Figure 55)}

\section{Species}

Mouse; Rat.

\section{Synonyms}

Developmental cyst; Thyroglossal duct cyst

\section{Pathogenesis/cell of origin}

Congenital finding/remnant of thyroglossal duct.

\section{Diagnostic Features}

- Small cystic or duct-like structure lined by follicles or simple cuboidal to columnar epithelium that may be ciliated.

- Often filled by mucinous material.
- Usually located along the ventral aspect of the larynx on the midline or in the thyroid gland.

Differential Diagnoses

Follicle, Cystic:

- Cystic or elongated follicles lined by a single layer of low cuboidal or flattened epithelium.

- May contain pale colloid.

- Often located at the periphery of the thyroid gland.

Cyst, Ultimobranchial:

- Lined by flattened squamous epithelium and contains laminated keratin.

\section{Comment}

Rare in rats and mice. The thyroglossal duct develops during downgrowth of the endoderm from the midline of the floor of the primitive pharynx and usually regresses. Occasionally, remnants of the duct persist within the thyroid gland.

References

(Chandra et al. 2013; Faccini et al. 1990; Frith et al. 2000;

Hardisty and Boorman 1990; Hardisty and Boorman 1999)

\section{Pigment (N) Thyroid Gland (Figure 56)}

Species

Mouse; Rat.

\section{Pathogenesis/cell of origin}

Accumulation of colored products derived from cellular breakdown, lipid peroxidation of cellular membranes, or chemicals or their metabolites.

\section{Diagnostic Features}

- Multiple types of pigment can accumulate in the follicular epithelial cells or colloid.

- Brown granular pigment is most common, and may stain positive for iron, lipofuscin, PAS stain, and occasionally for acid-fast stain.

\section{Comment}

Lipofuscin pigment of follicular cells increases with age or after prolonged thyroid gland stimulation. Some chemicals or their metabolites can cause deposition of pigment into the thyroid follicular epithelium (such as minocycline, which cause black discoloration of the thyroid gland).

\section{References}

(Chandra et al. 2013; Frith et al. 2000; Hardisty and Boorman 1999; Tajima et al. 1985; Ward and Reznik-Schüller 1980; Ward et al. 1979b)

Thyroid dysplasia (N) Thyroid Gland (Figures 57 and 58) Species

Rat. 
Synonyms

Vacuolar change; thyroid epithelial edema.

Pathogenesis/cell of origin

Degenerative change, congenital.

Diagnostic Features

- Thyroid gland may be enlarged.

- Enlarged follicular cells.

- Apically displaced nuclei.

- Large intracellular vacuole with homogeneous eosinophilic content located at the base of follicular epithelial cells.

- Diffuse, rarely focal or multifocal.

\section{Differential Diagnosis}

Hypertrophy, Follicular Cell:

- The nucleus is not apically displaced.

- No vacuoles at the base of the follicular epithelial cells.

\section{Comment}

Follicular dysplasia has been observed in dwarf and normal-sized Hannover Wistar GALAS rats. By electron microscopy, the rough endoplasmic reticulum was dilated, which displaced the normally basally located nucleus to an apical position. The probable genetic origin as well as the histologic features led to the term 'thyroid gland dysplasia'. Even though dysplasia is used as a diagnostic term, this entity is not associated with thyroid proliferative changes. Thyroid follicular cell (basilar) vacuolation is an acceptable term for the morphologic change seen with thyroid dysplasia. Rats with thyroid dysplasia and normal body growth may be more sensitive to carcinogens than normal thyroids.

\section{References}

(Abe et al. 2012; Doi et al. 2004; Kokoshima et al. 2014; Sakai et al. 2000; Shimoi et al. 2001; Takaoka et al. 1994; Weber et al. 2009)

\section{Ultimobranchial cyst (N) Thyroid Gland (Figure 59)}

Species

Mouse; Rat.

\section{Synonyms}

Developmental cyst; Squamous epithelial cyst.

\section{Pathogenesis/cell of origin}

Congenital finding/remnant of embryonic ultimobranchial duct.

\section{Diagnostic Features}

- May be single or multiple.

- Commonly located in the central portion of thyroid gland.

- Cystic or elongated duct-like structures lined by flat- tened squamous epithelium.

- Usually contains keratinaceous or cellular debris.

- The epithelium may be ciliated, particularly in mice.

\section{Differential Diagnoses}

Follicle, Cystic:

- Cystic or elongated follicles lined by a single layer of low cuboidal or flattened epithelium.

- May contain pale colloid.

- Often located at the periphery of the thyroid gland.

Persistent Thyroglossal Duct:

- Small cystic or duct-like structures lined by follicles or simple cuboidal or columnar epithelium which may be ciliated.

- Often filled by mucinous material.

- Usually located along the ventral midline aspect of the larynx or in the thyroid gland.

\section{Comment}

Common finding in rats. Occasionally ultimobranchial cysts form follicular structures that are lined by a mixture of flattened ultimobranchial epithelium and normal thyroid follicular epithelial cells, representing apparently areas of continuity between the ultimobranchial cyst and the thyroid parenchyma.

References

(Faccini et al. 1990; Frith et al. 2000; Hardisty and Boorman 1990; Hardisty and Boorman 1999)

\section{B. Proliferative Lesions}

\section{Hyperplasia, follicular cell (H) Thyroid Gland}

(Figures 60, 61, 62, and 63)

Species

Mouse; Rat.

Modifier

Focal; Cystic; Diffuse.

Pathogenesis/cell of origin

Follicular epithelial cells.

Diagnostic Features

- Increased number of follicular epithelial cells.

- A capsule is not present.

- Papillary infoldings into the colloid may be present, but growth pattern is not atypical.

- Cells are cuboidal or low columnar but some piling up may be present.

- Cytoplasm may be more eosinophilic or basophilic.

Focal:

- Consists of a focal area of hyperplastic follicles. 
- Margins may be indistinct.

- Compression of the adjacent thyroid tissue can be variable.

Focal, cystic:

- Follicles are enlarged and cystic.

- Follicular cells may form papillary projections or microfollicular structures in part of the cyst.

- Large colloid-filled follicles may cause compression of adjacent normal follicles.

Diffuse:

- Thyroid gland may be grossly enlarged.

- Follicles are frequently smaller with reduced colloid.

- Small papillary projections of follicular cells occasionally extend into the lumen.

- Limited piling up of cells.

- Epithelial cells are cuboidal to columnar (hypertrophic).

- Follicular cells are hyperchromatic without cellular atypia.

\section{Differential Diagnoses}

Follicle, Cystic:

- Large dilated colloid-filled follicles, sometimes originating from several confluent follicles.

- Epithelium is flattened.

- Epithelial projections are not present.

Adenoma, Follicular Cell:

- Compression of adjacent follicles.

- Proliferative pattern that is macro- or microfollicular.

- A capsule may be present.

- Well demarcated.

- Increased cytoplasmic basophilia may be present.

Hypertrophy, Follicular Cell:

- Increased height and volume of follicular cells.

- May occur alone or with hyperplasia.

\section{Comment}

Follicular thyroid hyperplasia may be diffuse or focal. Diffuse hyperplasia is typically a response to chronic stimulation, and expected to return to normal if the stimulus is withdrawn. Since diffuse hyperplasia represents a physiological response to increased serum TSH it is recommended that the modifier, diffuse, be used. Focal follicular hyperplasia is considered a preneoplastic lesion. Large cystic follicles may be ruptured and the broken septae can be misinterpreted as hyperplastic papillary features; however, cystic follicles are lined by flattened epithelium. The distinction between hyperplasia and adenoma is not always clear, and in rodents, progression from focal hyperplasia to adenoma is common. Hypertrophy and hyperplasia often occur together and are usually due to increased circulating thyroid stimulating hormone (TSH) concentration.

\section{References}

(Boorman and Elwell 1996; Botts et al. 1994; Burek 1978; Capen et al. 2001; Chandra et al. 2013; Frith and Heath 1984; Hardisty and Boorman 1990; Hardisty and Boorman 1999; Heath 1996b; Jokinen and Botts 1994; KaspareitRittinghausen et al. 1990; Murthy 1990; Napalkov 1990)

\section{Adenoma, follicular cell (B) Thyroid Gland}

(Figures 64, 65, 66, and 67)

Species

Mouse; Rat.

Modifiers

Follicular; Cystic; Papillary; Solid.

\section{Pathogenesis/cell of origin}

Follicular epithelial cells.

\section{Diagnostic Features}

- Well demarcated.

- Compression of the adjacent thyroid follicles is present.

- A capsule (complete or partial) may be present.

- Epithelium is single or multilayered (piling up).

- Proliferative growth pattern is microfollicular or macrofollicular.

- Cellular atypia may be present.

- Cells are cuboidal or columnar.

- Cells are eosinophilic or basophilic and may have nuclear basophilia. Some cells may have vacuolated cytoplasm.

- Nuclei are enlarged and oval or round and may have distinct nucleoli.

- Mitotic figures are uncommon.

- Size is not a criterion.

- Growth patterns are papillary, follicular, cystic and solid. Different growth patterns can be present in the same neoplasm. Animals may have multiple adenomas with different growth patterns.

Follicular:

- Small or regular-sized follicles.

Cystic:

- Dilated follicles (macrofollicular) in a well circumscribed area.

Papillary:

- Follicular cells form papillary projections in enlarged follicles.

- Papillae may have fibrous stroma.

Solid:

- Cells are arranged in solid sheets and densely packed nodules.

- Small follicles with minimal colloid may be present.

- Can resemble C-cell tumors. 


\section{Differential Diagnoses}

Follicle, Cystic:

- Large dilated colloid-filled follicle sometimes originating from several confluent follicles.

- Epithelium is flattened.

- Papillary infolding is not present.

Hyperplasia, Follicular Cell (Focal):

- Compression is variable, for example cystic or dilated follicles can compress adjacent thyroid tissue.

- Poorly demarcated from surrounding follicles.

- Capsule is not present.

- Papillary infolding may be present.

Carcinoma, Follicular Cell:

- Invasion or metastases are present.

- Not well demarcated.

- Cells may exhibit atypia or pleomorphism.

Adenoma, C-cell:

- Solid pattern with neuroendocrine packeting.

- Small colloid-filled follicles are not present.

- The C-cell origin can be confirmed by immunohistochemical demonstration of calcitonin.

\section{Comment}

Spontaneous thyroid follicular cell adenomas are rare in mice. There appears to be a continuum from hyperplasia to carcinoma in induced thyroid tumors. The differentiation between hyperplasia and adenoma is not well defined. Size is not a reliable criterion. Use of neoplasm modifiers (Follicular; Cystic; Papillary; Solid) is optional.

\section{References}

(Boorman and Elwell 1996; Botts et al. 1994; Burek 1978; Capen et al. 2001; Capen and Martin 1989; Frith and Heath 1984; Hardisty and Boorman 1990; Hardisty and Boorman 1999; Heath 1996b; Jokinen and Botts 1994; KaspareitRittinghausen et al. 1990; Murthy 1990; Napalkov 1990; Ohshima and Ward 1986; Pilling et al. 2007; Ward and Ohshima 1986)

\section{Carcinoma, follicular cell (M) Thyroid Gland}

(Figures 68, 69, and 70)

Species

Mouse; Rat.

Modifiers

Follicular; Papillary; Pleomorphic; Solid

Pathogenesis/cell of origin

Follicular epithelial cells of the thyroid gland.

\section{Diagnostic Features}

- Vascular, capsular, or intraglandular invasion is present.

- Metastasis may be present, usually to regional lymph nodes or the lungs.
- Cells may exhibit atypia or pleomorphism.

- Nuclei are enlarged or reduced in size, round or elongated, or sometimes clear.

- Mitotic figures may be present and often numerous.

- Fibrosis or scirrhous reaction, necrosis, mineralization, pigment deposits and cholesterol cleft formation may be present.

- Reactive fibrosis may be present at site of invasion.

- Growth patterns are follicular, papillary, pleomorphic, and solid, or a mixture of these.

Follicular:

- Irregular variable-sized follicles are lined with multiple layers of disorganized epithelium.

- Sometimes solid nodules or clusters of cells are present.

Papillary:

- Irregular papillary structures with one or multiple epithelial layers.

- Dense regions of the carcinoma may have tubular features.

Solid:

- Cells are arranged in sheets and nodules.

- Cells are polygonal and atypical.

- Can resemble C-cell tumors.

Pleomorphic:

- Pleomorphic, undifferentiated or anaplastic cells and nuclei.

- Cells can be large, spindle-shaped or multinucleate giant cells.

\section{Differential Diagnoses}

Adenoma, Follicular Cell:

- Well demarcated.

- Invasion or metastases are not present.

Carcinoma, C-cell:

- Solid carcinoma with neuroendocrine packeting.

- The tumor cells usually have the amphophilic tinctorial proprieties of C-cells.

- Small colloid-filled follicles or follicle-like structures are rare.

- The C-cell origin can be confirmed by immunohistochemical demonstration of calcitonin.

\section{Comment}

It is thought that follicular carcinomas usually represent progression from adenomas. Use of neoplasm modifiers (Follicular; Papillary; Pleomorphic; Solid) is optional.

\section{References}

(Boorman and Elwell 1996; Botts et al. 1994; Burek 1978; Capen et al. 2001; Capen and Martin 1989; Frith and Heath 1984; Hardisty and Boorman 1990; Hardisty and Boorman 1999; Heath 1996b; Jokinen and Botts 1994; KaspareitRittinghausen et al. 1990; Murthy 1990; Napalkov 1990; Pilling et al. 2007) 


\section{Hyperplasia, C-cell (H) Thyroid Gland}

(Figures 71, 72, 73, and 74)

Species

Mouse; Rat.

\section{Synonyms}

Hyperplasia, light cell; Hyperplasia, parafollicular cell.

\section{Modifiers}

Focal; Diffuse; Complex type.

\section{Pathogenesis/cell of origin}

Calcitonin-producing endocrine cells of the thyroid gland.

\section{Diagnostic Features}

Focal:

- Focal increase in $\mathrm{C}$ cells predominantly interfollicular but can also be intrafollicular.

- Not larger than the area of five average thyroid follicles.

- No compression of the adjacent tissue.

- A capsule is not present.

- Cells are arranged in solid nests.

- Stromal septa are not present.

- Cells are polygonal with pale to amphophilic cytoplasm.

- Cell borders are indistinct.

- Nuclei are round or oval.

Diffuse:

- Diffuse increase in C cells.

- No evidence of compression of the adjacent tissue.

- Cells are polygonal with pale to amphophilic cytoplasm.

- Cell borders are indistinct.

- Nuclei are round or oval.

Complex type (rare, only seen in the rat):

- Focal hyperplasia that also contains ganglion-like cells.

\section{Differential Diagnoses}

Adenoma, C-cell:

- Larger than the area of five average thyroid follicles.

- Compression may be present.

- A thin capsule may be present.

- Stromal septa may be present.

Adenoma, Follicular Cell (solid):

- Small, colloid-containing follicles are often present.

- Immunohistochemistry for thyroglobulin is positive and negative for calcitonin.

\section{Comment}

$\mathrm{C}$ cells in the rat and mouse are predominantly located in the central part of the thyroid gland. $\mathrm{C}$ cells normally increase with age in the rat, so the diagnosis of diffuse C-cell hyperplasia should be compared to age-matched, sex-matched controls. Spontaneous C-cell hyperplasia is rare in mice. $\mathrm{C}$ cells are not easily identified in mice and immunohistochemistry for calcitonin will increase the sensitivity for identification of C-cell hyperplasia in mice. Diffuse C-cell hyperplasia has a different pathogenesis compared to focal $\mathrm{C}$-cell hyperplasia, so the modifier, diffuse, should be used when the hyperplasia is diffuse. Focal C-cell hyperplasia is a preneoplastic lesion. Diffuse C-cell hyperplasia represents a physiological or pathophysiological response to stimulation of $\mathrm{C}$ cells, such as chronic hypercalcemia.

\section{References}

(Boorman et al. 1996; Botts et al. 1994; Burek 1978; Capen et al. 2001; Capen and Martin 1989; Frith and Heath 1984; Hardisty and Boorman 1990; Hardisty and Boorman 1999; Heath 1996b; Jokinen and Botts 1994; Kaspareit-Rittinghausen et al. 1990; Kiupel et al. 2008a; Martín-Lacave et al. 2002; Murthy 1990; Napalkov 1990; Pilling et al. 2007; Rosol 2013)

Adenoma, C-cell (B) Thyroid Gland (Figures 75, 76, and 77)

Species

Mouse; Rat.

\section{Synonyms}

Adenoma, parafollicular cell; Tumor, C-cell, benign; Adenoma, light cells.

\section{Modifiers}

Ganglioneuroma type; Complex type.

\section{Pathogenesis/cell of origin}

Calcitonin-producing endocrine cells of the thyroid gland.

\section{Diagnostic Features}

- Larger than the area of five average thyroid follicles.

- Well circumscribed.

- Compression of the adjacent tissue may be present.

- A thin capsule may be present.

- Cells are arranged in solid nests with neuroendocrine packeting.

- May contain scattered thyroid follicles.

- Angiectasis or hemorrhage may occur.

- Amyloid may be present in the stroma, especially in the mouse.

- Stromal septa may be present.

- Cells are polygonal with pale to amphophilic cytoplasm.

- Cell borders are indistinct.

- Nuclei are uniform round to oval.

- Mitotic figures are rare.

Ganglioneuroma type (rat):

- Consists exclusively of ganglion-like cells with a neurofibrillar eosinophilic matrix.

Complex type (rat):

- Consists of C cells and ganglion-like cells. 


\author{
Differential Diagnoses \\ Hyperplasia, C-cell (focal): \\ - Less than the area of five average thyroid follicles. \\ - No evidence of compression of the adjacent tissue. \\ - A capsule is not present. \\ - Stromal septa are not present. \\ Carcinoma, C-cell: \\ - Invasion of extrathyroidal tissue. \\ - Metastasis to regional lymph nodes or the lungs.
}

Adenoma, Follicular Cell (solid):

- Small colloid follicles are present.

- Immunohistochemistry for thyroglobulin is positive and negative for calcitonin.

\section{Comment}

The origin of $\mathrm{C}$ cells and the ganglion-like cells is the neural crest. Therefore, the complex type in the rat is a mixture of both cell types. The matrix contains neurites and some Schwann cells. The ganglion-like cells are immunohistochemically negative for calcitonin.

$\mathrm{C}$-cell adenomas are rare in mice.

\section{References}

(Boorman et al. 1996; Botts et al. 1991; Burek 1978; Capen et al. 2001; Capen and Martin 1989; Crissman et al. 1991; Hardisty and Boorman 1990; Hardisty and Boorman 1999; Jokinen and Botts 1994; Kaspareit-Rittinghausen et al. 1990; Kiupel et al. 2008a; Martín-Lacave et al. 2002; Murthy 1990; Napalkov 1990; Pilling et al. 2007; Rosol 2013; Van Zwieten et al. 1983; Ward et al. 1979a)

\section{Carcinoma, C-cell (M) Thyroid Gland}

(Figures 78, 79, and 80)

Species

Mouse; Rat.

\section{Synonyms}

Carcinoma, clear cell; Carcinoma, light cell; Carcinoma, medullary; Carcinoma, parafollicular cell; Tumor, C-cell, malignant.

\section{Pathogenesis/cell of origin}

Calcitonin-producing endocrine cells of the thyroid gland.

\section{Diagnostic Features}

- Thyroid gland, capsular or vascular invasion.

- Large carcinomas may replace most of the normal thyroid gland.

- Distant metastases to regional lymph nodes and lung may be present, especially in large neoplasms.

- Cells are arranged in solid nests and sheets with neuroendocrine packeting that may be separated by fibrous stroma.

- Cells are polygonal with pale to amphophilic cytoplasm.

- Cell borders are indistinct.
- Nuclei are uniform, round to oval, and may be spindleshaped.

- Cellular pleomorphism may be present.

- Number of mitotic figures is variable.

- Central necrosis may be present.

- May contain hemorrhage.

- Amyloid may be present in the stroma, especially in the mouse.

- A partial capsule may be present.

Complex type (rat):

- Consists of C cells and ganglion-like cells.

Differential Diagnoses

Adenoma, C-cell:

- No vascular or capsular invasion.

- No metastasis.

Carcinoma, Follicular Cell:

- The solid type of follicular carcinoma usually has some follicle formation, which should not be confused with scattered thyroid follicles entrapped in C-cell neoplasms. Differentiation can be confirmed using immunohistochemistry for thyroglobulin and calcitonin.

\section{Comment}

Generally the cellular morphology in C-cell neoplasms is similar to focal C-cell hyperplasia. Focal C-cell hyperplasia, adenoma and carcinoma represent a progression of disease. $\mathrm{C}$-cell carcinoma is rare in mice.

In the rare, complex $\mathrm{C}$-cell carcinoma of the rat, the ganglion cells are not considered malignant.

\section{References}

(Boorman et al. 1996; Botts et al. 1991; Burek 1978; Capen et al. 2001; Capen and Martin 1989; Crissman et al. 1991; Hardisty and Boorman 1990; Hardisty and Boorman 1999; Jokinen and Botts 1994; Kaspareit-Rittinghausen et al. 1990; Kiupel et al. 2008a; Martín-Lacave et al. 2002; Murthy 1990; Napalkov 1990; Pilling et al. 2007; Rosol 2013; Van Zwieten et al. 1983; Ward et al. 1979a) 


\section{Parathyroid GLAND}

\section{A. Nonproliferative Lesions}

\section{Amyloid (N) Parathyroid Gland \\ Species \\ Mouse; Rat.}

\section{Synonyms}

Amyloidosis; Amyloid deposition.

\section{Pathogenesis/cell of origin}

Degenerative change characterized by extracellular deposition of polypeptides derived from immunoglobulin or serum proteins.

\section{Diagnostic Features}

- Diffuse interstitial (extracellular) amorphous pale eosinophilic material.

- Green birefringence using polarized light with positive Congo red stain. Positive with Thioflavine T stains or specific immunohistochemical antibodies.

\section{Comment}

Spontaneous systemic amyloidosis is common in mice, especially CD-1 and C57BL/6 strains. Amyloidosis can also be induced by administration of Freund's adjuvant and casein.

\section{References}

(Chandra et al. 2013; Frith and Chandra 1991; Venalis et al. 2005)

Angiectasis (N) Parathyroid Gland (Figure 81)

Species

Mouse; Rat.

\section{Synonyms}

Hemangiectasis; Hemangiectasia; Teleangiectasis.

\section{Pathogenesis/cell of origin}

Vasculature of the parathyroid gland.

\section{Diagnostic Features}

- Small to large, blood-filled spaces, lined by well differentiated endothelial cells.

\section{Differential Diagnosis}

Congestion:

- Diffuse dilatation of blood filled vessels not distorting the architecture of the tissue.

\section{Comment}

This is an uncommon lesion that may occur in conjunction with focal or diffuse hyperplasia or adenoma.

\section{References}

(Frith et al. 2000; Seely and Hildebrand 1990)

\section{Atrophy (N) Parathyroid Gland}

Species

Mouse; Rat.

\section{Synonyms}

Chief cell atrophy.

Pathogenesis/cell of origin

Chief cells of the parathyroid gland.

\section{Diagnostic Features}

- Small parathyroid glands.

- Small chief cells with high nuclear to cytoplasmic ratio.

- Chief cells have little eosinophilic cytoplasm.

\section{Comment}

Rats and mice with increased serum ionized calcium concentration or excessive vitamin D supplementation will have suppression of parathyroid hormone (PTH) synthesis and subsequent atrophy of the parathyroid chief cells. The area of chief cell cytoplasm is proportional to the production and secretion of PTH.

References

(Capen and Rosol 1993; Rosol et al. 1986)

Cyst (N) Parathyroid Gland (Figure 82)

Species

Mouse; Rat.

Synonyms

Kursteiner's cyst; Kursteiner's duct cyst.

\section{Pathogenesis/cell of origin}

Congenital.

\section{Diagnostic Features}

- Lined by cuboidal or columnar epithelium, which is variably ciliated.

- May be empty or filled with eosinophilic or mucinous material.

- May be single, multiple or multiloculated.

- Mild compression of adjacent tissue may be evident.

\section{Comment}

The cysts are formed by remnants of the third and fourth pharyngeal pouches, which are the origins of the parathyroid glands and thymus. Parathyroid cysts have been reported secondary to chronic exposure to chemicals in rats, as a result of destruction of chief cells and retention of cellular debris. 


\section{References}

(Capen 1996c; Capen and Rosol 1989; Chamanza et al. 2010; Chandra et al. 2013; Frith et al. 2000; Frith and Fetters 1996a; Hardisty and Boorman 1999; Kittel et al. 1996a; Seely and Hildebrand 1990)

\section{Ectopic tissue, parathyroid (N) Parathyroid Gland}

\section{Species}

Mouse; Rat.

Pathogenesis/cell of origin

Congenital.

\section{Diagnostic Features}

- Small nodules of parathyroid tissue usually within thymic tissue.

\section{Comment}

Due to the close association of parathyroid and the thymus during embryological development ectopic parathyroid may be found in the thymus. Genetic ablation experiments suggest that small foci of parathyroid chief cells in the thymus may be normal in the mouse. Parathyroid tumors may arising in the precardiac mediastinum from these ectopic rests.

\section{References}

(Capen 1975; Chandra et al. 2013; Frith et al. 2000; Frith and Fetters 1996a; Günther et al. 2000; Hardisty and Boorman 1999; Kittel et al. 1996a, b; Seely and Hildebrand 1990)

\section{Ectopic tissue, thymus (N) Parathyroid Gland}

Species

Mouse; Rat.

\section{Pathogenesis/cell of origin}

Congenital.

\section{Diagnostic Features}

- Thymic tissue located adjacent to or inside the parathyroid gland.

- Consists mostly of thymic lymphocytes.

- May contain pale staining clusters of epithelial cells and/ or Hassall's corpuscles.

- Well demarcated.

\section{Differential Diagnoses}

Infiltrate, Inflammatory Cell (Lymphoid):

- Infiltration of lymphocytes without epithelial cells.

- Poorly demarcated.

Lymphoma, Malignant, Systemic Infiltration:

- Consists of malignant lymphocytes.

- Usually not restricted to the parathyroid gland.

- Other organs affected.

\section{Comment}

Due to the close association of parathyroid and the thymus during embryological development, ectopic thymic tissue may be found in the parathyroid gland.

References

(Capen 1975; Frith et al. 2000; Hardisty and Boorman 1999; Kittel et al. 1996a, b)

Fibrosis (N) Parathyroid Gland (Figure 83)

Species

Mouse; Rat.

Pathogenesis/cell of origin

Collagen deposition by fibroblasts following inflammation, necrosis, or hemorrhage.

Diagnostic Features

- Increased amounts of mature collagen in the capsule or interstitium of the gland.

- Fibroblasts are usually not prominent.

\section{Comment}

The amount of capsular and interstitial connective tissue varies between animals and increases with age. Fibrosis is used when the amount of connective tissue is considered greater than normal variation.

References

(Burek 1978; Frith et al. 2000; Kittel et al. 1996a)

\section{Hypertrophy (N) Parathyroid Gland}

Species

Mouse; Rat.

Modifiers

Diffuse; Focal.

Pathogenesis/cell of origin

Chief cells of the parathyroid gland.

Diagnostic Features

Diffuse:

- Chief cells are enlarged with increased cytoplasm.

- The nuclear:cytoplasm ratio is decreased.

- Cytoplasm is eosinophilic and may contain small clear vacuoles.

- Both glands are affected.

Focal:

- Single or small clusters of enlarged chief cells that may have large clear cytoplasmic vacuoles (water-clear cells)

- No compression of the surrounding parathyroid tissue.

Differential Diagnoses

Hyperplasia, Diffuse: 
- Chief cells are increased in number and enlarged.

- Both parathyroid glands have homogeneous uniform enlargement.

- Enlarged parathyroid glands may compress the adjacent thyroids

Hyperplasia, Focal:

- Chief cells are increased in number in single or multifocal areas within one or both glands.

- Poorly demarcated from the surrounding parenchyma.

- No or minimal compression of the surrounding parathyroid tissue.

- The cytoplasm to nuclear ratio can be increased or decreased compared to normal chief cells.

- Cytoplasm is eosinophilic and may be vacuolated.

\section{Comment}

Diffuse hypertrophy of chief cells represents an early stage of physiological stimulation of the chief cells, usually due to hypocalcemia or decreased calcitriol (active form of vitamin D). Continued stimulation of the chief cells will lead to diffuse hyperplasia.

\section{References}

(McInnes 2012)

\section{Infiltrate, inflammatory cell (N) Parathyroid Gland}

\section{Species}

Mouse; Rat.

\section{Pathogenesis/cell of origin}

Accumulation of small number of inflammatory cells; uncertain pathogenesis or local immune response.

\section{Diagnostic Features}

- Small numbers of inflammatory cells within the interstitium.

- Usually consists of mononuclear cells but there may be small numbers of neutrophils.

- Focal or multifocal.

\section{Differential Diagnosis}

Inflammation:

- Usually more extensive.

- Typically associated with tissue damage and/or vascular changes.

\section{References}

(Altenähr and Jenke 1974; Frith et al. 2000)

\section{Inflammation (N) Parathyroid Gland}

\section{Species}

Mouse; Rat.

\section{Synonyms}

Parathyroiditis.

\section{Pathogenesis/cell of origin}

Inflammatory change.

\section{Diagnostic Features}

- Typically a widespread inflammatory cell infiltrate with lymphocytes, plasma cells and macrophages and rare neutrophils.

- Associated with tissue damage and/or vascular changes.

- Can be acute or chronic.

\section{Differential Diagnosis}

Infiltrate, Inflammatory Cell:

- Usually mononuclear cells but may have small numbers of neutrophils.

- Usually focal or multifocal.

\section{Comment}

This is a rare lesion. Experimental parathyroiditis in rats was reported to result from passive immunization.

\section{References}

(Altenähr and Jenke 1974; Frith et al. 2000)

\section{Multinucleate giant cells (N) Parathyroid Gland}

(Figure 84)

Species

Rat.

Synonyms

Syncytial giant cells.

Pathogenesis/cell of origin

Degenerative change or fixation artifact.

Diagnostic Features

- Large, multinucleated syncytial cells containing dense eosinophilic cytoplasm.

- Nuclei are hyperchromatic, small, and oval.

- Uncommon change observed near the periphery of the gland.

\section{Differential Diagnosis}

Hyperplasia, Focal:

- Single or multiple nodular lesions composed of closely packed chief cells.

- Does not contain multinucleated cells.

\section{Comment}

Syncytial giant cells are thought to result from disruption and fusion of the cell membrane of several chief cells. Ultrastructural studies indicated degenerative changes. The significance of this change is unknown. Wild and Setoguti (1995) considered them to be fixation artifacts. 


\section{References}

(Botts et al. 1991; Capen and Rosol 1989; Frith et al. 2000; Rosol et al. 2013; Seely and Hildebrand 1990; Wild and Setoguti 1995)

\section{B. Proliferative Lesions}

Hyperplasia (N) Parathyroid Gland (Figures 85, 86, and 87)

Species

Mouse; Rat.

Modifiers

Focal; Diffuse.

Pathogenesis/cell of origin

Chief cells of the parathyroid gland.

Diagnostic Features

Focal:

- Chief cells are increased in number in single or multifocal areas within one or both glands.

- Poorly demarcated from the surrounding parenchyma.

- No or minimal compression of the surrounding parathyroid tissue.

- A capsule is not present.

- The cytoplasm to nuclear ratio can be increased or decreased compared to normal chief cells.

- Cytoplasm is eosinophilic and may be vacuolated.

Diffuse:

- All parathyroid chief cells have homogeneous uniform enlargement.

- The chief cells are increased in number and enlarged (hypertrophied).

- Adjacent thyroid follicles may be compressed.

- Both glands have similar growth pattern.

- Cytoplasm is eosinophilic and may be vacuolated.

- Chief cells are polygonal or may be elongated.

- Nuclei are round or elongated.

\section{Differential Diagnoses}

Hypertrophy, Diffuse:

- Chief cells are enlarged with increased cytoplasm.

- The cytoplasm to nuclear ratio is increased.

- Cytoplasm is eosinophilic and may contain small clear vacuoles.

- Both glands are affected

Adenoma:

- Solitary nodule.

- Compression of adjacent normal chief cells.

- Well demarcated.

- Capsule may be present.

\section{Comment}

Diffuse hyperplasia is reversible if the stimulus is withdrawn. Dietary and renal diseases that alter calcium metabolism are etiological factors. Diffuse hyperplasia is frequently associated with chronic renal disease and secondary hyperparathyroidism. Fibrous osteodystrophy of bone can occur in animals with secondary hyperparathyroidism.

Focal hyperplasia is usually non-functional and represents a pre-neoplastic change.

\section{References}

(Bomhard 1993; Botts et al. 1994; Botts et al. 1991; Capen 1990; Capen et al. 2001; Hardisty and Boorman 1999; Kamino et al. 1996b; Kaspareit-Rittinghausen et al. 1989; Lewis and Cherry 1982; Rosol and Capen 1989; Seely and Hildebrand 1990)

\section{Adenoma (N) Parathyroid Gland (Figure 88)}

Species

Mouse; Rat.

Pathogenesis/cell of origin

Chief cells of the parathyroid gland.

Diagnostic Features

- Solitary nodule in the parathyroid gland.

- Well demarcated.

- Compression of the adjacent chief cells is present.

- A capsule or partial capsule may be present.

- The cytoplasm to nuclear ratio can be increased or decreased compared to normal chief cells.

- The growth pattern is usually solid, but papillary, acinar or cystic pattern can occur.

- Nuclear pleomorphism or mitoses may be present.

Differential Diagnoses

Hyperplasia, Focal:

- No compression.

- Poorly demarcated.

- No capsule.

- Chief cells may have increased or decreased cytoplasm.

Carcinoma:

- Vascular and/or capsular invasion is present.

- Metastases rarely occur.

\section{Comment}

Parathyroid neoplasms are uncommon in rats and rare in mice. Adenomas are usually nonfunctional in rats. Adenomas derived from ectopic parathyroid tissue can rarely occur in the thoracic mediastinum. Parathyroid adenomas do not represent a progression of diffuse hyperplasia, but likely represent progression from focal hyperplasia. 


\section{References}

(Bomhard 1993; Botts et al. 1994; Botts et al. 1991; Burek 1978; Capen 1990; Capen et al. 2001; Hardisty and Boorman 1999; Kamino et al. 1996a; Lewis and Cherry 1982; Libutti et al. 2003; Rosol and Capen 1989; Seely and Hildebrand 1990)

\section{Carcinoma (N) Parathyroid Gland (Figure 89)}

Species

Mouse; Rat.

\section{Pathogenesis/cell of origin}

Chief cells of the parathyroid glands.

\section{Diagnostic Features}

- Vascular, capsular, or intrathyroidal invasion is present.

- Metastases may occur, particularly to the regional lymph nodes or lungs.

- Usually as large or larger than the normal gland.

- Compression of the adjacent chief cells is present.

- Nuclear pleomorphism and mitosis are usually present.

- Growth pattern is usually solid.

- Proliferating chief cells are polygonal or elongated.

\section{Differential Diagnosis}

Adenoma:

- Invasion and metastases are not present.

\section{Comment}

Parathyroid neoplasms are uncommon in rats and rare in mice. Invasion of tumor cells occur in rat parathyroid carcinoma, but metastasis has not been described.

\section{References}

(Bomhard 1993; Botts et al. 1994; Botts et al. 1991; Burek 1978; Capen 1990; Capen et al. 2001; Hardisty and Boorman 1999; Kamino et al. 1996a; Lewis and Cherry 1982; Pace et al. 2003; Rosol and Capen 1989; Seely and Hildebrand 1990)

\section{AdRENAL GLAND}

\section{A. Nonproliferative Lesions}

Amyloid (N) Adrenal Gland (Figure 90)

Species

Mouse; Rat.

Synonyms

Amyloidosis; Amyloid degeneration; amyloid infiltration.

Pathogenesis/cell of origin

Degenerative change.

\section{Diagnostic Features}

- Amyloid deposits are primarily in the inner cortex surrounding the medulla.

- Extracellular/intercellular faintly eosinophilic, acellular amorphous deposits usually starting in zona reticularis; deposits may largely replace this zone in severe cases.

- Green birefringence using polarized light with Congo red stain.

- Positive with Thioflavin T stain or specific immunohistochemical antibodies.

\section{Differential Diagnoses}

Necrosis:

- Congo red, Thioflavin $\mathrm{T}$ and immunohistochemical staining negative.

- Disruption of cellular architecture.

- Nuclear pyknosis, karyolysis, or karyorrhexis.

Fibrosis:

- Fibrillary appearance with small numbers of fibroblasts.

- Congo red, Thioflavin $\mathrm{T}$ and immunohistochemical staining negative.

- Positive collagen staining (van Gieson's, Picrosirius red and Masson-trichrome).

\section{Comment}

Adrenal amyloidosis is usually observed as a component of systemic amyloidosis. Adrenal amyloidosis is common in aging mice of certain strains, such as CD-1 mice. In contrast, adrenal amyloidosis is rarely present in $\mathrm{B} 6 \mathrm{C} 3 \mathrm{~F} 1$ mice and rats. In F344 rats, it has been reported in the absence of systemic amyloidosis. In the adrenal gland, amyloid deposition usually start in the zona reticularis and may progress to efface much of this zone in severe cases.

\section{References}

(Chandra et al. 2013; Frith and Chandra 1991; Hamlin II and Banas 1990; Nyska and Maronpot 1999; Sass 1996b; Yarrington 1996) 


\section{Angiectasis (N) Adrenal Gland (Figure 91)}

Species

Mouse; Rat.

Synonyms

Peliosis; hemangiectasis; hemangiectasia; telangiectasis; telangiectasia.

Pathogenesis/cell of origin

Vascular change.

Diagnostic Features

- Blood-filled spaces lined by endothelium (capillary dilatation).

- May occur in cortex or medulla.

\section{Differential Diagnoses}

Cystic Degeneration:

- Endothelium-lined spaces may be suggestive of a vascular change, but evidence of cellular degeneration is also present.

- Differentiation from cystic degeneration with hemorrhage may be difficult, as both findings may occur together.

Hemangioma:

- Expansive proliferation of well-differentiated endothelial cells forming variably sized blood-filled spaces.

Hemangiosarcoma:

- Infiltrative proliferation of poorly-differentiated endothelial cells forming haphazard vascular spaces.

- Moderate to marked cellular atypia and invasion of adjacent tissues with or without metastasis.

\section{Comment}

Angiectasis is a common lesion in aging female rats, and it is rarely found in mice. This lesion may be associated with inflammatory, degenerative, hyperplastic and/or neoplastic diseases. Congestion with hemangiectasis and dilatation of intercellular spaces may be related to stress and/or exogenous administration of adrenocorticotropic hormone (ACTH). In both mice and rats, dilation of cortical capillaries and resultant angiectasis can occur secondary to progressive age-related cortical cell atrophy or loss.

\section{References}

(Frith et al. 2000; Greaves 2012a; Hamlin II and Banas 1990; Nyska and Maronpot 1999)

\section{Atrophy, cortical (N) Adrenal Gland (Figure 92)}

\section{Species}

Mouse; Rat.

\section{Synonyms}

Cortical atrophy.
Pathogenesis/cell of origin

Disturbance of growth/cortical cells.

Diagnostic Features

- Bilateral or unilateral reduction in cortical thickness, usually in the zona fasciculata and reticularis with loss of cells and/or reduction in cytoplasmic volume.

- Bilateral decrease or loss of lipid vacuolation may be present.

- Variable disruption of the normal architectural cortical arrangement.

- Pyknosis and lipofuscin deposition may be present.

- The cytoplasm is usually eosinophilic. The amount of cytoplasm is normal or mildly decreased with no or few cytoplasmic fat vacuoles.

- The nuclei may be densely basophilic or heterochromatic.

\section{Differential Diagnoses}

Hypoplasia, Cortical:

- Congenital lesion; no evidence of cellular degeneration such as pyknosis or lipofuscin deposition.

Sectioning Artifact:

- Tangential section through the adrenal gland.

\section{Comment}

Bilateral atrophy usually results from decreased adrenocorticotropic hormone (ACTH) due to destructive lesions in the pituitary gland. It may also result from perturbation of the renin-angiotensin system (affecting the zona glomerulosa) or due to exogenous or endogenous corticosteroid excess (atrophy of the zona fasciculata and reticularis). Unilateral atrophy may occur in the presence of a corticosteroid-secreting neoplasm in the contralateral adrenal cortex.

\section{References}

(Chandra et al. 2013; Frith et al. 2000; Greaves 2012a; Greaves and Faccini 1992; Hamlin II and Banas 1990; Nyska and Maronpot 1999; Rosol et al. 2001)

Cyst, cortical (N) Adrenal Gland (Figure 93)

Species

Mouse.

\section{Pathogenesis/cell of origin}

Congenital or acquired malformation of cortical cells.

\section{Diagnostic Features}

- Located in the cortex.

- True cysts are lined by a single layer of flattened or cuboidal to columnar epithelium that may be ciliated.

Differential Diagnosis

Angiectasis:

- Blood or protein-rich fluid in dilated vessels. 


\section{Comment}

Cysts are rarely seen in mice and are not described in rats. They are reported to occur in the $\mathrm{B} 6 \mathrm{C} 3 \mathrm{~F} 1$ strain of mice.

\section{References}

(Nyska and Maronpot 1999)

Degeneration, cystic (N) Adrenal Gland (Figure 94)

Species

Mouse; Rat.

Synonyms

Cystic change.

\section{Pathogenesis/cell of origin}

Sequela of cell swelling or fatty degeneration of cortical cells.

\section{Diagnostic Features}

- Cell loss (decreased number of cortical cells in comparison to surrounding parenchyma) with formation of cystic spaces that may be filled with blood or proteinaceous fluid.

- Usually focal and well circumscribed, but less frequently involves extensive cell loss in most of the cortex progressing to large cystic or blood-filled spaces.

- Compression of adjacent tissue may be present due to enlargement or swelling of cortical cells.

\section{Differential Diagnoses}

Angiectasis:

- Cystic degeneration may be difficult to differentiate from angiectasis with hemorrhage.

- Angiectasis is usually lined by endothelial cells.

Vacuolation, Increased, Cortical, Focal:

- No or little evidence of cell degeneration.

- May be associated with cystic or blood-filled spaces.

Hyperplasia, Cortical, Focal:

- Increased number of cortical cells without prominent vacuolation.

Adenoma, Cortical:

- Compression of the adjacent tissue with loss of parenchymal architecture and disruption of radial arrangement of cell cords.

- Increased adrenal cortical cell numbers.

- Cystic degeneration may occur in cortical adenomas to various degrees.

\section{Comment}

Occurs predominantly in aging female rats, particularly the Sprague-Dawley strain. Due to the expansile nature of the lesion, adjacent tissue is often compressed or necrotic and may involve the entire adrenal cortex. Cystic cortical degeneration is considered to be a continuum of severe fo- cal cortical vacuolation, in which there is significant cell loss and resulting cavitation and blood-filled spaces. The tissue adjacent to cystic degeneration should be carefully examined since cystic degeneration can also occur within hyperplastic and neoplastic lesions. Cystic degeneration may be associated with contralateral adrenal atrophy. This lesion is rarely seen in mice.

\section{References}

(Chandra et al. 2013; Elmore et al. 2013; Frith et al. 2000; Greaves and Faccini 1992; Hamlin II and Banas 1990; Laast et al. 2014; Nyska and Maronpot 1999; Yarrington 1996)

\section{Ectopic tissue, adrenocortical (N) Adrenal Gland} (Figure 95)

\section{Species}

Mouse; Rat.

\section{Synonyms}

Accessory cortical tissue, adrenal extracortical nodule, hamartoma, adrenocortical rest.

\section{Pathogenesis/cell of origin}

Congenital lesion/cortical cells.

\section{Diagnostic Features}

- Presence of concomitant adrenocortical tissue outside or immediately inside the adrenal capsule.

- Most commonly found in the retroperitoneal fat adjacent to the adrenal gland or kidney, but can be found anywhere in the abdominal cavity.

- Composed of normal cortex either detached from the adrenal gland, or attached to the gland and may be separated by a fibrous capsule.

- Medullary tissue is not present.

- May lack the distinct zonal arrangement of the adrenal cortex.

- No Compression of adjacent parenchyma.

- A complete or incomplete capsule may be present.

- Features of cellular atypia and vascular or capsular invasion are not present.

\section{Differential Diagnoses}

Adenoma, Cortical:

- A discrete mass composed of sheets or lobules of well differentiated cortical epithelial cells with compression of the adjacent tissue.

Carcinoma, Cortical:

- An expansile proliferation of cortical cells with cellular atypia and invasion of adjacent parenchyma.

Hyperplasia, Subcapsular Cell (Mouse):

- Composed of large polygonal cells with clear cytoplasm (type B), mixed with fusiform, basophilic cells (type A), oriented along the capsular surface and generally con- 
tinuous with adjacent cortical parenchyma.

\section{Comment}

This is a congenital (spontaneous) background lesion, which has not been associated with experimental procedures. It is composed of normal cortex and the cells exhibit characteristics of the zona glomerulosa, fasciculata, and/ or reticularis, but lack medullary tissue. The ectopic tissue may be either completely detached from the existing adrenal gland, or attached but separated from the adrenal gland by a fibrous capsule

\section{References}

(Belloni et al. 1989; Chandra et al. 2013; Faccini et al. 1990; Frith et al. 2000; Greaves 2012a; Greaves and Faccini 1992; Hamlin II and Banas 1990; Nyska and Maronpot 1999; Parker and Valerio 1996a; Sass 1996a)

\section{Fibrosis (N) Adrenal Gland (Figure 96)}

\section{Species}

Mouse; Rat.

\section{Pathogenesis/cell of origin}

Collagen deposition by fibroblasts following inflammation, necrosis, or hemorrhage.

\section{Diagnostic Features}

- Replacement of normal tissue architecture by organized dense, mature collagen, interspersed by fibroblasts or granulation tissue.

- Positive for collagen stains such Masson trichrome, van Gieson, or Picrosirius red.

\section{Differential Diagnoses}

Amyloidosis:

- Adrenal amyloid deposits are primarily in the inner cortex surrounding the medulla.

- Extracellular/intercellular faintly eosinophilic, amorphous acellular deposits usually starting in zona reticularis; deposits may largely replace this zone in severe cases.

- Green birefringence using polarized light with Congo red stain.

\section{Sarcoma/Fibrosarcoma:}

- Infiltrative proliferation of bundles and intersecting fascicles of atypical spindle cells that efface the underlying parenchyma and produce collagen.

\section{Comment}

Adrenal fibrosis is uncommon finding in the mouse and rat and can rarely be observed spontaneously in old rats and mice. This lesion may be associated with a variety of neoplastic, vascular, inflammatory, or infectious diseases, and may occur in a regional, focal, or peripheral pattern, depending on the underlying cause. In cases where fibro- plasia or granulation tissue is active and exuberant, the lesion should be differentiated from a spindle cell neoplasm. Fibrosis may also be seen as a component of an organizing thrombus, in which case it is usually associated with hemosiderin and/or mineral deposition.

\section{References}

(Rosol et al. 2001)

\section{Hematopoiesis, extramedullary (N) Adrenal Gland}

(Figure 97)

Species

Mouse; Rat.

Synonyms

Hematopoietic cell proliferation; EMH.

\section{Pathogenesis/cell of origin}

Extramedullary hematopoietic cells.

Diagnostic Features

- Consists of small random clusters of erythrocytic and granulocytic precursors and/or megakaryocytes, but usually the erythrocytic component predominates or is the only cell type present.

- Usually present in adrenal cortex

- Can be focal or multifocal.

\section{Differential Diagnoses}

Infiltrate, Inflammatory Cell:

- Depending on the type of infiltrate, mature granulocytes, lymphocytes or mixed inflammatory cell infiltrates are present.

Inflammation:

- Inflammation may be neutrophilic, lymphoplasmacytic, histiocytic, or granulomatous, and is usually associated with generalized systemic disease or an extension of peritonitis.

- Cortical cell degeneration or loss may be apparent.

Lymphoma/Leukemic Infiltration:

- These systemic malignancies usually infiltrate several organs and are characterized by the presence of (uniform) neoplastic cells.

\section{Comment}

Extramedullary hematopoiesis can be seen in the adrenal cortex, especially in situations where the hematopoietic system has been stimulated. When it is found in the adrenal gland, usually prominent extramedullary hematopoiesis is also present in the spleen.

\section{References}

(Frith et al. 2000; Greaves and Faccini 1992; Nyska and Maronpot 1999) 


\section{Hemorrhage (N) Adrenal Gland (Figure 98)}

Species

Mouse; Rat.

Pathogenesis/cell of origin

Vascular change.

\section{Diagnostic Features}

- Extravasated erythrocytes between cords of the adrenal cortex.

- Hemosiderin-laden macrophages may be present if the hemorrhage is chronic.

\section{Differential Diagnosis}

Angiectasis:

- Blood-filled spaces lined by endothelium (capillary dilatation).

\section{Comment}

Hemorrhage is an uncommon lesion and may overlap with angiectasis, thrombosis and cortical necrosis. Hemorrhage often occurs with cystic degeneration in rats.

\section{References}

(Greaves 2012a; Rosol et al. 2001)

\section{Hypertrophy, cortical, diffuse (N) Adrenal Gland}

(Figure 99 (control) and Figure 100)

\section{Species}

Mouse; Rat.

\section{Pathogenesis/cell of origin}

Stimulation of growth / cortical cells.

\section{Diagnostic Features}

- Increased cytoplasmic volume and usually enlargement of the nucleus.

- Cytoplasm of hypertrophic cells may be densely eosinophilic with reduced cytoplasmic vacuoles, finely granular, or filled with small lipid vacuoles (microvesicular).

- Usually bilateral.

- Diffuse hypertrophy is characterized by increased cortical thickness affecting one to several cortical zones. The zona fasciculata and reticularis are most often affected, but all three cortical zones can be involved, and differentiation between the three zones may be less distinct.

\section{Differential Diagnoses}

Vacuolation, Cortical, Increased, Diffuse:

- Cytoplasmic (lipid) vacuolation (macrovesicular) of cortical cells is diffusely distributed throughout one or several cortical zones. This may lead to an increased thickness of the cortex.

Hyperplasia, Cortical, Diffuse:

- Diffuse enlargement of one or more cortical zones due to increased number of cortical cells.

\section{Comment}

The diagnosis of diffuse hypertrophy should be used for enlarged cortical cells without increased vacuolation. The diagnosis of vacuolation, increased, diffuse should be used for cortical cells that are enlarged due to greater numbers and/or size of cytoplasmic lipid vacuoles. The diagnosis of diffuse hyperplasia should be used when there is an increase in cortical cell number. Diffuse hypertrophy and hyperplasia can occur together.

Hypertrophy with dense eosinophilic cytoplasm is usually caused by increased secretion of ACTH with stress or disease or exogenous administration of ACTH. A variety of xenobiotics can inhibit steroid synthesis and may not only produce increased vacuolation with accumulation of lipid but also adaptive hypertrophy and even hyperplasia and neoplasia.

\section{References}

(Everds et al. 2013; Greaves 2012a; Hamlin II and Banas 1990; Harvey and Sutcliffe 2010; Rebuffat et al. 1992)

\section{Hypertrophy, cortical, focal (N) Adrenal Gland}

(Figure 101)

Species

Mouse; Rat.

\section{Pathogenesis/cell of origin}

Stimulation of growth.

\section{Diagnostic Features}

- Distinct focus of increased cell size in the adrenal cortex, usually in the zona fasciculata or reticularis.

- Cell cytoplasm is usually eosinophilic and finely granular.

- Cytoplasmic vacuoles may be present.

- Nuclei may be increased in size.

- No nuclear atypia.

- No compression of adjacent cortical cells.

\section{Differential Diagnosis}

Hyperplasia, Cortical, Focal:

- Distinct focus with increased number of cortical cells, which may be large cells.

- May have mild compression of adjacent cortical cells.

- Nuclear atypia may be present.

\section{Comment}

It may be challenging to differentiate focal hypertrophy and focal hyperplasia of the adrenal cortex, especially when the hyperplastic cells are enlarged. In the past, the terms basophilic or eosinophilic focus may have been used for either focal cortical hypertrophy or hyperplasia. Focal hypertrophy may also be present in a multifocal pattern. 


\section{References}

(Frith et al. 2000; Greaves 2012a; Hamlin II and Banas 1990; Laast et al. 2014; Nyska and Maronpot 1999; Rosol et al. 2001)

Infiltrate, inflammatory cell (N) Adrenal Gland (Figure 102) Species

Mouse; Rat.

\section{Pathogenesis/cell of origin}

Accumulation of small number of inflammatory cells; uncertain pathogenesis or local immune response.

\section{Diagnostic Features}

- Small numbers of inflammatory cells in the interstitium.

- Usually consists of mononuclear cells but may have small numbers of neutrophils depending on the stimulus.

- Focal or multifocal.

\section{Differential Diagnoses}

Hematopoiesis, Extramedullary:

- Consists of immature erythrocytic or granulocytic cells, and/or megakaryocytes. No tissue damage is present.

Inflammation:

- Usually more extensive and typically associated with tissue damage and/or vascular changes.

\section{References}

(Frith et al. 2000; Nyska and Maronpot 1999; Tucker 1997)

Inflammation (N) Adrenal Gland (Figures 103, 104, and 105)

Species

Mouse; Rat.

Synonyms

Adrenalitis.

\section{Pathogenesis/cell of origin \\ Inflammatory change.}

\section{Diagnostic Features}

- Multifocal to diffuse infiltration of predominantly granulocytes in acute inflammation, plasma cells and lymphocytes in chronic inflammation, and histiocytes in histiocytic or granulomatous inflammation.

- Inflammation is usually classified as acute, subacute, chronic, or chronic-active (with both acute and chronic components).

- Degeneration or loss of cortical cells may occur.

- Typically associated with tissue damage and/or vascular changes.

\section{Differential Diagnoses}

Infiltrate, Inflammatory Cell:

- Small number of inflammatory cells.
- No tissue damage is present.

Hematopoiesis, Extramedullary:

- Consists of immature erythrocytic or granulocytic cells, and/or megakaryocytes.

- No tissue damage is present.

Lymphoma/Leukemia Infiltration:

- These systemic malignancies usually infiltrate several organs, often cause tissue damage, and are characterized by the presence of (uniform) neoplastic cells.

\section{Comment}

Inflammation of the adrenal is rare and is usually associated with systemic disease or an extension of peritonitis.

References

(Frith et al. 2000; Nyska and Maronpot 1999)

Mineralization (N) Adrenal Gland (Figure 106)

Species

Mouse; Rat.

Synonyms

Calcification.

Pathogenesis/cell of origin

Degeneration/necrosis of cortical cells followed by deposition of mineral.

\section{Diagnostic Features}

- Inorganic mineral substance which is homogenous to granular and densely basophilic

- Multifocal or diffuse.

- Intracellular or extracellular.

- May occur subsequent to necrosis or hemorrhage.

\section{Differential Diagnosis}

Osseous Metaplasia:

- Focus of metaplastic bone formation in the adrenal cortex.

- May be immature woven bone or lamellar bone without bone marrow formation, including extramedullary hematopoiesis.

\section{Comment}

Dystrophic mineralization occurs in the adrenal cortex often following necrosis or hemorrhage/thrombosis. If mineralization is associated with necrosis, thrombosis, or hemorrhage the diagnosis of mineralization is optional. Adrenal medullary mineralization has been induced in rats following chronic exposure to chlorodibromomethane.

\section{References}

(Frith et al. 2000; Hamlin II and Banas 1990; Hoenerhoff et al. 2016) 
Necrosis (N) Adrenal Gland (Figures 107 and 108)

Species

Mouse; Rat.

Pathogenesis/cell of origin

Cell death.

Diagnostic Features

- Loss of cellular architecture.

- Nuclear pyknosis, karyorrhexis, or karyolysis.

- Cellular swelling, hypereosinophilia, fragmentation, or shrinkage.

- May be associated with hemorrhage, cystic degeneration, inflammation and mineralization.

- Can be single-cell, focal, multifocal or diffuse.

- Can occur in any zone, but usually occurs in the zona fasciculata and/or reticularis.

\section{Differential Diagnoses}

Autolysis:

- Diffuse dissolution of tissue architecture and loss of differential staining without evidence of necrosis, apoptosis, or disruption of tissue structure.

Degeneration, Cystic:

- Focal loss of cortical cells secondary to cellular hypertrophy and vacuolation with formation of cystic spaces filled with blood or proteinaceous fluid.

Atrophy:

- Thinning of the cortex due to decreased cortical cell size and/or number without associated inflammation, cellular debris, hemorrhage, or cystic degeneration.

\section{Comment}

Necrosis may be associated with inflammatory, degenerative, and/or neoplastic disease. Spontaneous adrenal necrosis occurs uncommonly in rats and mice. In rats, necrosis often occurs secondary to thrombosis, hemorrhage or systemic neoplasia such as mononuclear cell leukemia, or inflammatory or degenerative lesions. Some chemical agents can induce necrosis of the adrenal cortex (such as 7,12-dimethylbenz(a)anthracene). Necrosis can overlap with hemorrhage and mineralization. Only the predominant finding in the lesion should be diagnosed.

\section{References}

(Chandra et al. 2013; Frith et al. 2000; Greaves 2012a; Hamlin II and Banas 1990; Huggins and Morii 1961; Rosol et al. 2013; Rosol et al. 2001; Tucker 1997; Yarrington 1996)

Osseous metaplasia (N) Adrenal Gland (Figure 109)

Species

Mouse; Rat.

\section{Pathogenesis/cell of origin}

Degenerative change.

\section{Diagnostic Features}

- Focus of metaplastic bone formation in the adrenal cortex.

- May be immature woven bone or lamellar bone.

- May have extramedullary hematopoiesis.

\section{Differential Diagnoses}

Myelolipoma:

- Benign neoplasm composed of adipose cells with a variable amount of intermixed hematopoietic tissue (similar to bone marrow). Osseous metaplasia may be present in a myelolipoma.

Mineralization:

- Inorganic mineral substance which is homogenous to granular and densely basophilic

- Intracellular or extracellular.

\section{Comment}

Osseous metaplasia is usually a spontaneous (background) finding in rodents. The cause of osseous metaplasia has not been identified. The presence of metaplastic bone alone should be diagnosed as osseous metaplasia. When associated with bone marrow formation and extramedullary hematopoiesis, the diagnosis of a myelolipoma is preferred.

\section{References}

(Hamlin II and Banas 1990)

\section{Pigment (N) Adrenal Gland (Figure 110)}

Species

Mouse; Rat.

\section{Synonyms}

Lipofuscin(osis), ceroid, brown atrophy, brown degeneration, wear-tear pigment.

\section{Pathogenesis/cell of origin}

Accumulation of colored products derived from cellular breakdown or lipid peroxidation of cellular membranes of adrenocortical cells.

\section{Diagnostic Features}

- Yellow to brown granular pigment, usually in cells of the zona reticularis and/or histiocytes at the junction of the zona reticularis and medulla.

- Pigment stains positive for lipofuscin and is usually PAS-positive and sudanophilic; may be acid fast and autofluorescent.

\section{Differential Diagnosis}

Hemosiderosis:

- Hemosiderin-laden macrophages also occur frequently 
in the same region. Special stains for iron (e.g., Prussian blue) may be used to confirm hemosiderin.

\section{Comment}

Small amounts of lipofuscin are commonly observed in aged rats and mice. However, its presence in young animals may be indicative of increased cellular organelle turnover or impaired cell metabolism (inhibition of steroid synthesis). Lipofuscin pigmentation may be associated with severe hormone-induced atrophy. Dietary deficiency of antioxidants such as vitamin $\mathrm{E}$ enhances the production and storage of lipofuscin pigment. In some mouse strains, especially BALB/c mice, it is a common finding. Its severity can be enhanced by the administration of estrogens and adrenocorticosteroids.

In adult and aged mice a persistent X-zone might be present and represented by hypertrophic and fused adrenal cortical cells or macrophages containing lipid globules and pigmented cytoplasm (lipofuscin/ceroid pigment).

\section{References}

(Chandra et al. 2013; Frith 1996b; Frith et al. 2000; Greaves 2012a; Hamlin II and Banas 1990; Nyska and Maronpot 1999; Parker and Valerio 1996d; Rosol et al. 2013; Tucker 1997)

\section{Thrombus (N) Adrenal Gland (Figure 111)}

Species

Mouse; Rat.

Pathogenesis/cell of origin

Vascular change.

\section{Diagnostic Features}

- Acute thrombi are characterized as solid, laminated intravascular accumulations of fibrin, platelets, erythrocytes and inflammatory cells, often attached to the endothelial surface of a blood vessel (commonly observed in the adrenal cortex with secondary expansion of vascular sinusoids).

- Chronic thrombi are characterized by organization of acute thrombi, evidenced by production of dense collagen matrix and recannulation by capillaries. Hemosiderin may be present.

- Thrombosis may be associated with infarction, fibrosis, and collapse of the surrounding parenchyma, which may be the only histopathologic evidence of thrombosis.

- Thrombi may contain large numbers of neutrophils, especially if associated with sepsis.

\section{Differential Diagnoses}

Angiectasis:

- Diffuse or focal dilation of blood vessels that may be associated with secondary thrombosis.
Postmortem Clot:

- Lacks lamination and contains few to no leukocytes.

\section{Comment}

Adrenal thrombosis may result from chronic angiectasis, local tissue or vascular damage, or systemic vasculopathy. It is an uncommon incidental finding in the mouse and rat adrenal gland. Thrombosis also may be associated with systemic inflammation or sepsis. When mild and focal, adrenal thrombosis may be iatrogenic and sporadic, whereas when diffuse and severe, may be a sign of systemic inflammatory or infectious disease or widespread multi-organ vasculopathy.

References

(Hamlin II and Banas 1990)

Vacuolation, cortical, increased, diffuse (N) Adrenal Gland (Figure 112)

Species

Mouse; Rat.

Synonyms

Fatty change; lipidosis; lipoid hyperplasia.

Pathogenesis/cell of origin

Degeneration or lipid retention of the cortical cells.

Diagnostic Features

- Small, foamy and optically clear vesicles (microvesicular) or large vacuoles (macrovesicular) in cytoplasm of cortical cells.

- The cells may be enlarged and/or the number of cortical cells may be increased.

- Diffuse cytoplasmic vacuolation of cortical cells is associated with involvement of one or more zones of the cortex, usually including the zona fasciculata.

- Cellular enlargement caused primarily by the accumulation of lipid is diagnosed as vacuolation and not hypertrophy.

- Usually bilateral.

\section{Differential Diagnoses}

Hypertrophy, Cortical, Diffuse:

- Enlarged cortical cells with eosinophilic or finely granular cytoplasm; vacuolation is not a dominant feature.

Hyperplasia, Cortical, Diffuse:

- Increased number of cortical cells, and vacuolation is usually not a dominant feature.

\section{Comment}

Diffuse microvesicular vacuolation is a normal pattern in the adrenal cortex. In some strains there is a slight difference between genders. The vacuoles that occur in adrenal cortical cells with increased vacuolation can represent lipid 
vacuoles, swollen mitochondria, or phospholipidosis. Electron microscopy can be helpful to differentiation the nature of the vacuoles. A variety of xenobiotics can increase lipid deposition and macrovacuolar vacuolation in the adrenal cortex usually by inhibition of steroid synthesis. This may not only produce an accumulation of lipid and retention of a xenobiotic, but also adaptive hypertrophy, hyperplasia and even neoplasia. There may be a concurrent increase in vacuolation of the interstitial steroid-producing cells of the ovary.

\section{References}

(Greaves 2012a; Hamlin II and Banas 1990; Laast et al. 2014; Landes et al. 1996; Nyska and Maronpot 1999; Rosol et al. 2001; Tucker 1997)

\section{Vacuolation, cortical, increased, focal (N) Adrenal Gland}

(Figure 113)

\section{Species}

Mouse; Rat.

\section{Synonyms}

Focal fatty change; lipidosis; degeneration vacuolar; clear cell focus.

\section{Pathogenesis/cell of origin \\ Degeneration/cortical cell.}

\section{Diagnostic Features}

- Discrete, well demarcated increased cytoplasmic vacuolation of cortical cells with gradual transition to the surrounding cortex at the periphery.

- Small, foamy and clear vesicles (microvesicular) or large vacuoles (macrovesicular) in cytoplasm of cortical cells.

- The cells may be enlarged.

- Usually occurs in one or more zones of the cortex, such as the zona fasciculata or glomerulosa.

- Individual cells may be increased in size, but the number of cells within the lesion is not increased.

- Slight compression of adjacent cortical cells may be present.

- Marked compression and increase in size beyond the regular cortical thickness may occur when combined with degenerative changes such as sinusoid dilatation and cystic degeneration.

\section{Differential Diagnoses}

Hypertrophy, Cortical, Focal:

- Enlarged cortical cells with eosinophilic or finely granular cytoplasm, and vacuolation is not a dominant feature.

Hyperplasia, Cortical, Focal:

- Increased number of cortical cells.

- Vacuolation is usually not a dominant feature of these lesions. Hyperplastic cortical cells can be smaller or larger than normal.
Degeneration, Cystic:

- A continuum of cortical vacuolation resulting in cell loss with formation of cystic spaces that may be filled with blood.

\section{Comment}

Focal increased vacuolation is a common, spontaneous change in aged rats. Fat vacuolation may also occur in cortical cells in proliferative lesions and may be prominent in cortical adenomas.

\section{References}

(Hamlin II and Banas 1990; Laast et al. 2014; Nyska and Maronpot 1999; Rosol et al. 2001)

Vacuolation, cortical, decreased, diffuse (N) Adrenal Gland Species

Mouse; Rat.

Synonyms

Diffuse lipid depletion; diffuse hypovacuolation.

Pathogenesis/cell of origin

Degeneration or physiological response of cortical cells.

\section{Diagnostic Features}

- The cytoplasm is more dense and homogeneous eosinophilic than usual with a reduction in lipid vacuoles.

- The cortical cells are normal in size, or decreased in size due to lipid depletion.

- May be associated with involvement of one or more zones of the cortex, usually including the zona fasciculata and reticularis.

- The nuclei of the cortical cells are normal.

- Usually bilateral.

\section{Differential Diagnoses}

Hypertrophy, Cortical, Diffuse:

- Enlarged cortical cells with eosinophilic finely granular or compact cytoplasm, decreased vacuolation is a concomitant feature.

Hyperplasia, Cortical, Diffuse:

- Increased number of cortical cells; decreased vacuolation may be feature of hyperplasia.

\section{Comment}

Decreased vacuolation may represent a result of ACTHinduced lipid depletion, stress or administration of xenobiotics. Cortical cells are normal in size. It the cortical cells are increased in size then they should be considered hypertrophied. Chronic stress or conditions that significantly increase serum ACTH concentrations would also be expected to induce diffuse cortical hypertrophy and hyperplasia. 


\section{References}

(Greaves 2012a; Landes et al. 1996; Nyska and Maronpot 1999; Rosol et al. 2013)

\section{Vacuolation, cortical, decreased, focal (N) Adrenal Gland}

(Figure 114)

Species

Mouse; Rat.

\section{Synonyms}

Basophilic focus; eosinophilic focus; focus of cytoplasmic alteration.

Pathogenesis/cell of origin

Degeneration/cortical cell.

\section{Diagnostic Features}

- The change in vacuolation affects a discrete area of the adrenal cortex and usually occurs in the zona fasiculata.

- Cortical cells are small, normal-sized, or mildly enlarged.

- Cytoplasm has no or few fat vacuoles.

- Cytoplasm is eosinophilic or basophilic.

- Nuclei are heterochromatic or dark.

- There is no compression of surrounding tissue

\section{Differential Diagnosis}

Hyperplasia, Cortical, Focal:

- Increased number of cortical cells.

- Cortical cells may be smaller or larger and have cytoplasmic tinctorial changes compared to surrounding cells.

- The region of hyperplasia is rounded or angular, and there may be mild compression of adjacent cells.

\section{Comment}

Focal decreased vacuolation of the adrenal cortex occurs in adult to aged rats. It represents a focal (or multifocal) change in metabolic status rather than a proliferative lesion and differentiation from hyperplasia can be confirmed with the use of proliferation markers, such as KI-67, PCNA or BrDU.

\section{References}

(Nyska and Maronpot 1999)

\section{Persistant X-zone (N) Adrenal Gland (Figure 115)}

Species

Mouse.

Synonyms

Impaired X-zone involution.

\section{Pathogenesis/cell of origin}

Abnormal persistence of fetal/neonatal adrenal cortical cells adjacent to the adrenal medulla.

\section{Diagnostic Features}

- Incomplete and delayed regression of the X-zone compared to control mice of the same sex, strain and age.

- Persistent X-zone cells may be non-vacuolated or less vacuolated than control mice.

Normal Regression of the X-Zone

- Normal neonatal mice: X-zone consists of small, basophilic adrenal cortical cells between the mature zona fasiculata and medulla

- Normal pubertal males: X-zone cells undergo fatty degeneration with enlarged cells containing single to multiple fat globules. There may be vascular dilatation and congestion.

- Normal females during first pregnancy: X-zone cells undergo fatty or vacuolar degeneration with apoptosis, single-cell necrosis, and fusion of cells. There may be vascular dilatation and congestion.

- Normal adult and aged mice: A remnant of the X-zone is represented by hypertrophic and fused adrenal cortical cells or macrophages containing lipid globules and cytoplasmic pigment (lipofuscin or ceroid pigment).

\section{Comment}

The zonation of the adrenal cortex in mice is complete at birth with a zona glomerulosa and fasiculata. The zona reticularis is not seen morphologically in mice. The X-zone is a region of basophilic adrenal cortical cells adjacent to the medulla that is present in neonatal mice and may represent cells derived from primordial fetal adrenal cells. The cells contain lipid droplets and other characteristics of steroid-producing cells. The function of the X-zone is unknown. The X-zone persists for variable lengths of time after birth depending on sex, strain and pregnancy status. In male mice, the X-zone undergoes fatty degeneration near puberty. In females, the X-zone continues to increase in size to reach a maximum at about 9 weeks. It regresses slowly with age and rapidly upon the first pregnancy. The X-zone regresses during the first pregnancy or pseudopregnancy by vacuolar or fatty degeneration. The thickness of the X-zone, the rate of regression, and the type of degeneration varies between strains and sources of mice and is genetically controlled. It is important not to misinterpret the degeneration associated with normal agerelated regression of the X-zone in mice as a lesion. It is not recommended that X-zone involution or degeneration be reported when it represents the normal aging process. Gonadectomy can prevent $\mathrm{X}$-zone regression and exogenous testosterone or progesterone can induce X-zone degeneration and regression. It has been reported that obese (ob/ob) mice had delayed regression of the X-zone.

\section{References}

(Hershkovitz et al. 2007; Janat and Shire 1987; Jones 1952; Naeser 1975; Nyska and Maronpot 1999; Rosol et al. 2013) 


\section{B. Proliferative Lesions}

\section{Hyperplasia, cortical, diffuse (H) Adrenal Gland}

Species

Mouse; Rat.

\section{Pathogenesis/cell of origin}

Adrenocortical cell.

\section{Diagnostic Features}

- Diffuse increase in the number of cortical cells in one or more cortical zones resulting in an increased cortical thickness.

- Cortical cells may be normal in size or enlarged

- Cytoplasm of hyperplastic cells may be eosinophilic with reduced cytoplasmic vacuoles, finely granular, or filled with small lipid vacuoles (microvesicular) that are similar to normal-sized lipid vacuoles.

\section{Differential Diagnoses}

Hypertrophy, Cortical, Diffuse:

- Enlargement of the cytoplasm and usually the nucleus.

- Cytoplasm of hypertrophic cells may be densely eosinophilic with reduced cytoplasmic vacuoles, finely granular, or filled with small lipid vacuoles (microvesicular) that are similar to normal-sized lipid vacuoles.

- Usually bilateral.

Vacuolation, Cortical, Increased, Diffuse:

- Cytoplasmic (lipid) vacuolation (macrovesicular) of cortical cells diffusely distributed throughout one or several cortical zones. This may increase the thickness of the cortical zones.

\section{Comment}

Diffuse hyperplasia can represent a physiological response to increased serum ACTH (such as occurs with chronic stress) or a change induced by xenobiotics. Diffuse hyperplasia is usually associated with an increase in adrenal weight. Diffuse hypertrophy may precede diffuse hyperplasia and both may occur together.

\section{References}

(Botts et al. 1994; Capen et al. 2001; Dunn 1970; Engeland and Levay-Young 1999; Everds et al. 2013; Faccini et al. 1990; Frith et al. 2000; Frith and Ward 1988b; Hamlin II and Banas 1990; Landes et al. 1996; Laroque et al. 1997; Nyska and Maronpot 1999; Rosol et al. 2013)

\section{Hyperplasia, cortical, focal (H) Adrenal Gland}

(Figures 116 and 117)

\section{Species}

Mouse; Rat.
Pathogenesis/cell of origin

Adrenocortical cell.

\section{Diagnostic Features}

- Adrenocortical cells are increased in number focally, and they may be smaller or larger than surrounding cortical cells.

- Most often located in the zona fasciculata, but may occur in any cortical zone.

- Focal or multifocal.

- No or minimal compression of the adjacent cortical tissue.

- The shape is usually rounded, but in some cases may be angular.

- The cytoplasm of hyperplastic cortical cells may be comparable to the surrounding cells or may exhibit tinctorial differences (eosinophilic, basophilic, or amphophilic).

- Cytoplasmic vacuolation may be present.

- Cellular or nuclear atypia, karyomegaly or polyploidy may be present.

- Not larger than the normal width of the cortex.

- Not intermingled with type A (spindle) cells (mouse).

\section{Differential Diagnoses}

Adenoma, Cortical:

- Distinct compression of the adjacent tissue.

- Architecture (radial arrangement of the cords) is not maintained.

- May be larger than the regular width of the cortex.

Hyperplasia, Subcapsular Cell (Mouse):

- Composed of large polygonal cells with clear cytoplasm (type B), admixed with fusiform, basophilic cells (type A).

- In or extending from a subcapsular location.

Hyperplasia, Medullary, Focal:

- Lipid droplets are not present.

- Cytoplasm is basophilic.

- Medullary cells are positive by immunohistochemistry for tyrosine hydroxylase, chromogranin A, synaptophysin, and neuron-specific enolase.

Vacuolation, Cortical, Increased, Focal:

- Focal vacuolization is characterized by a discrete zone of cortical cells containing (fat) vacuoles with gradual transition to the surrounding cortex. Individual cells may be increased in size, but the number of cells within the lesion is not increased.

- Focal cytoplasmic vacuolation of cortical cells is associated with involvement of one or more zones of the cortex, usually including the zona fasciculata or glomerulosa.

- Slight compression may be present; marked compression and increase in size beyond the regular cortical thickness may occur when combined with degenerative changes such as sinusoid dilatation and ballooning vacuolation. 
- Small, foamy and clear vesicles (microvesicular) or large vacuoles (macrovesicular) in cytoplasm of cortical cells.

- The cells may be enlarged.

Vacuolation, Cortical, Decreased, Focal:

- The change in vacuolation affects a discrete area the adrenal cortex and usually occurs in the zona fasiculata.

- Cortical cells are small, normal-sized, or mildly enlarged.

- Cytoplasm has no or few fat vacuoles and is eosinophilic or basophilic.

- Nuclei are heterochromatic or dark.

- There is no compression of surrounding tissue.

Degeneration, Cystic:

- Cell loss with formation of cystic spaces that may be filled with blood or proteinaceous fluid.

- Compression of adjacent tissue may be present.

\section{Comment}

Cellular atypia may occur in focal cortical hyperplasia and does not require a separate diagnosis. In the past, the terms basophilic, eosinophilic, or clear cell focus may have been used for focal cortical hyperplasia.

\section{References}

(Botts et al. 1994; Capen et al. 2001; Dunn 1970; Engeland and Levay-Young 1999; Faccini et al. 1990; Frith et al. 2000; Frith and Ward 1988b; Hamlin II and Banas 1990; Landes et al. 1996; Laroque et al. 1997; Nyska and Maronpot 1999; Patterson et al. 1995; Rosol et al. 2013; Strandberg 1996c)

Adenoma, cortical (B) Adrenal Gland (Figures 118 and 119)

Species

Mouse; Rat.

\section{Pathogenesis/cell of origin}

Adrenocortical cell.

\section{Diagnostic Features}

- Well-circumscribed nodule or mass with distinct compression of the adjacent cortical tissue.

- May be larger than the normal width of the adrenal cortex.

- Thin connective tissue capsule may be present.

- Architecture (radial arrangement of the cortical cords) is not maintained.

- Cellular pattern may be cords, trabeculae or solid clusters of cells.

- Cells may be smaller or enlarged and may exhibit tinctorial differences (rat).

- Usually composed of enlarged, eosinophilic or amphophilic cells (mouse).

- Cytoplasmic vacuolation (lipid droplets), angiectasis, hemorrhage, or thrombosis may be present.

- Cellular atypia may be present.

- Mitotic figures may be present.

\section{Differential Diagnoses}

Hyperplasia, Cortical, Focal:

- No compression or only slight compression of the adjacent tissue.

- Cortical architecture is maintained.

- Not larger than the regular width of the adrenal cortex.

Vacuolation, Cortical, Increased, Focal:

- Discrete zone of cortical cells containing (fat) vacuoles, with gradual transition to the surrounding cortex at the periphery. Slight compression may be present. Marked compression and increase in size beyond the regular cortical thickness may occur when combined with degenerative changes such as sinusoid dilatation and ballooning vacuolation.

- May involve one or more zones of the cortex, usually including the zona fasciculata or glomerulosa.

- Individual cells may be increased in size, but the number of cells within the lesion is not increased.

- Small, foamy and clear vesicles (microvesicular) or large vacuoles (macrovesicular) in cytoplasm of cortical cells.

Degeneration, Cystic:

- Cell loss with formation of cystic spaces that may be filled with blood or proteinaceous fluid

- Usually focal and well circumscribed

- Compression of adjacent tissue may be present.

Adenoma, Subcapsular (Mouse):

- Composed of large polygonal cells with clear cytoplasm (type B) admixed with fusiform, basophilic cells (type A).

- Subcapsular location.

Pheochromocytoma, Benign:

- Lipid droplets are not present.

- Cytoplasmic basophilia.

- Sinusoids and capillaries are often prominent and may be dilated.

- Tumor cells are immunohistochemically positive for tyrosine hydroxylase, chromogranin A, synaptophysin, and neuron-specific enolase.

Carcinoma, Cortical:

- Invasive growth into and beyond the surrounding adrenal tissue or distant metastasis is present.

\section{Comment}

If differentiation between focal hyperplasia and adenoma cannot be made on the basis of the criteria listed above, an adrenocortical proliferative lesion larger in size than the regular thickness of the adrenal cortex is typically interpreted to be an adenoma. Cortical adenomas in mice are rare compared to subcapsular hyperplasia and neoplasms.

\section{References}

(Botts et al. 1994; Capen et al. 2001; Dunn 1970; Duprat et al. 1990; Faccini et al. 1990; Frith et al. 2000; Frith and 
Ward 1988b; Hamlin II and Banas 1990; Heath 1996a; Laroque et al. 1997; Nyska and Maronpot 1999; Patterson et al. 1995; Rosol et al. 2013; Strandberg 1996b; Yarrington and O’Neal Johnston 1992)

\section{Carcinoma, cortical (M) Adrenal Gland}

(Figures 120, 121, and 122)

\section{Species}

Mouse; Rat.

\section{Pathogenesis/cell of origin}

Adrenocortical cell.

\section{Diagnostic Features}

- Invasive growth into surrounding adrenal tissue, beyond the adrenal capsule and/or distant metastasis.

- Neoplastic cells are organized in thickened trabeculae, sheets or solid clusters with disruption of normal architecture.

- Cytoplasm is usually eosinophilic or amphophilic.

- Cellular atypia and pleomorphism are usually present.

- Mitotic figures may be numerous.

- Vacuolation, cystic degeneration, necrosis, angiectasis or hemorrhage may be present.

\section{Differential Diagnoses}

Adenoma, Cortical:

- Invasive growth or metastasis are not present.

Carcinoma, Subcapsular (Mouse):

- Composed of large polygonal cells with clear cytoplasm, occasionally admixed with fusiform, basophilic cells (type A).

Pheochromocytoma, Malignant:

- Cytoplasmic basophilia.

- Cytoplasmic lipid droplets are not present.

- Prominent blood vessels.

- Tumor cells are immunohistochemically positive for tyrosine hydroxylase, chromogranin A, synaptophysin, and neuron-specific enolase.

\section{Comment}

Cortical atrophy of the adjacent or contralateral adrenal cortex supports the diagnosis of a functional cortical neoplasm. Cortical carcinomas are rare in mice.

\section{References}

(Botts et al. 1994; Capen et al. 2001; Dunn 1970; Duprat et al. 1990; Faccini et al. 1990; Hamlin II and Banas 1990; Heath 1996a; Laroque et al. 1997; Nyska and Maronpot 1999; Patterson et al. 1995; Rosol et al. 2013; Strandberg 1996a; Yarrington and O'Neal Johnston 1992)

\section{Hyperplasia, subcapsular cell (H) Adrenal Gland}

(Figure 123)

Species

Mouse.

\section{Modifiers}

Type A; Type B; Mixed type.

\section{Pathogenesis/cell of origin}

Derived from undifferentiated progenitor cells in the subcapsular region of the adrenal cortex.

\section{Diagnostic Features}

- Proliferation occurs in the subcapsular region with expansion parallel to the capsule and towards the medulla in a wedge-shaped manner.

- Proliferative cells consist of two types: Type A cells are densely cellular, small, oval to spindle-shaped with scant basophilic cytoplasm and are free of lipid vacuoles. Type $\mathrm{B}$ cells are large and polygonal with clear cytoplasm and small lipid vacuoles.

- The type A and type B cells are intermingled. The descriptive modifier used is based on the predominant cell type (> 70\%). The mixed type of hyperplasia consists of both A and B cells in similar proportions and is the most common type of hyperplasia.

- Focal, multifocal to circumferential.

- Focal hyperplasia may slightly bulge the surface of the adrenal gland.

- No or minimal compression of the underlying cortex.

- Does not grow into or beyond the capsule.

- Mitotic figures are rare.

- The width of the cortex is not increased.

\section{Differential Diagnoses}

Adenoma, Cortical:

- Circumscribed lesion with compression of the adjacent adrenal cortex, which may be larger than the normal width of the cortex. Note that some older mice have cortical atrophy, which can emphasize the size of subcapsular hyperplasia lesions.

Hyperplasia, Cortical, Focal:

- Composed of polygonal, non-spindle cells, usually eosinophilic or amphophilic cytoplasm.

- Cellular morphology is typical of cortical cells.

- Foci usually arise within the zona fasciculata (not a surface-oriented lesion), but may also occur in the zona glomerulosa or reticularis.

\section{Comment}

Subcapsular hyperplasia is a common spontaneous lesion in aging mice. Gonadectomy, hormone alterations, and housing conditions (crowding and isolation) may increase the incidence of this lesion. Differentiation between focal subcapsular hyperplasia and subcapsular adenoma based 
on lesion size is arbitrary and challenging.

\section{References}

(Capen et al. 2001; Dunn 1970; Faccini et al. 1990; Goodman 1996; Greaves 2012a; Nyska and Maronpot 1999; Rosol et al. 2013)

\section{Adenoma, subcapsular cell (B) Adrenal Gland}

(Figures 124, 125, and 126)

Species

Mouse.

Modifiers

Type A; Type B; Mixed type.

Pathogenesis/cell of origin

Derived from undifferentiated progenitor cells in the subcapsular region of the adrenal cortex.

\section{Diagnostic Features}

- A mass that bulges the surface of the adrenal gland and occurs in the subcapsular region of the adrenal cortex.

- Mild to marked compression of the underlying or adjacent cortex.

- May be wider than the width of the normal adrenal cortex.

- The proliferative cells consist of two types: Type A cells are densely cellular, small, oval to spindle-shaped with scant basophilic cytoplasm and are free of lipid vacuoles. Type B cells are large and polygonal with clear cytoplasm and small lipid vacuoles.

- Some adenomas have irregular, expansile growth outside of the capsule and form small nodules of A, B, or mixed A/B cells separated by fine fibrovascular stroma.

- The type A and type B cells are intermingled. The descriptive modifier used is based on the predominant cell type $(>70 \%)$. The mixed type of hyperplasia consists of both $\mathrm{A}$ and $\mathrm{B}$ cells in similar proportions and is the most common type of adenoma.

- Mitotic figures are rare.

- May arise as a distinct Type B cell proliferation within subcapsular cell hyperplasia.

\section{Differential Diagnoses}

Hyperplasia, Subcapsular Cell:

- There is no or minimal compression of the adjacent cortex.

- Often wedge-shaped.

- Not larger than the normal width of the adrenal cortex.

Carcinoma, Subcapsular Cell:

- Presence of capsular invasion or distant metastases.

- Tumor cell atypia, pleomorphism and mitotic figures.

Adenoma, cortical:

- Circumscribed lesion with compression of the adjacent adrenal cortex.

- May be larger than the normal width of the cortex.
- Some older mice have cortical atrophy, which can emphasize the size of the subcapsular hyperplasia.

\section{Comment}

Expansile growth beyond the adrenal capsule by an adenoma must be differentiated from invasion of the capsule and surrounding tissues that is present in a carcinoma. Differentiation between focal subcapsular hyperplasia and subcapsular adenoma can be difficult for borderline lesions.

\section{References}

(Capen et al. 2001; Dunn 1970; Faccini et al. 1990; Frith and Dunn 1994; Goodman 1996; Greaves 2012a; Heath 1996a; Nyska and Maronpot 1999; Rosol et al. 2013)

\section{Carcinoma, subcapsular cell (M) Adrenal Gland}

(Figure 127)

Species

Mouse.

\section{Modifiers}

Type A; Type B; Mixed type.

\section{Pathogenesis/cell of origin}

Derived from undifferentiated progenitor cells in the subcapsular region of the adrenal cortex.

\section{Diagnostic Features}

- Presence of distinct invasion into surrounding tissues or vessels or distant metastases, especially to the lungs.

- Tumor cell atypia, pleomorphism and mitotic figures are present.

- Cells are organized in nests, ribbons or cords.

- The modifiers are used according to the predominant A or B cell type (>70\%).

- Mixed type: No predominant cell type is present.

\section{Differential Diagnoses}

Adenoma, Subcapsular Cell:

- Absence of invasion of the capsular or surrounding tissues.

- No metastases.

Carcinoma, Cortical:

- Composed of polygonal, non-spindle cells, usually with eosinophilic or amphophilic cytoplasm.

\section{Comment}

Metastases have not been reported to occur spontaneously for subcapsular cell tumors in intact mice, but may occur in gonadectomized mice. Metastases have been reported to occur from Type A cells (Nyska and Maronpot 1999). The lung is the most common site for metastasis (Frith and Dunn 1994). It may be challenging to differentiate a large adenoma that expands beyond the capsule from a well differentiated carcinoma. 


\section{References}

(Capen et al. 2001; Faccini et al. 1990; Frith and Dunn 1994; Nyska and Maronpot 1999)

\section{Hyperplasia, medullary, diffuse (H) Adrenal Gland}

\section{Species}

Mouse; Rat.

\section{Pathogenesis/cell of origin} Adrenal medullary (chromaffin) cell.

\section{Diagnostic Features}

- Medulla is enlarged.

- Architecture is generally maintained, but the number of chromaffin cells is increased.

- No or minimal compression of the surrounding cortical tissue.

- The nests and cords of medullary cells may be wider than in the normal medulla.

- Cells may be smaller or larger and have tinctorial differences in the cytoplasm.

- Nuclear-cytoplasmic ratio may be increased.

- Mitotic figures are rare.

- Frequently associated with a decreased cortical to medullary ratio.

\section{Differential Diagnosis}

Pheochromocytoma, Benign:

- Distinct compression at the periphery is present.

- Architecture is not maintained.

\section{Comment}

Medullary hyperplasia is much more common in rats compared to mice, and the incidence is strain dependent. Strains with a high incidence include the Wistar, Gunn, Long-Evans, Fisher 344, and Sprague-Dawley. Sections through the center of the adrenal gland are required for accurate diagnosis. Cells in medullary hyperplasia can consist of both epinephrine (adrenaline) and norepinephrine (noradrenaline) cells, whereas pheochromocytomas usually consist of norepinephrine cells. Rat adrenal medullary cells readily respond to mitogenic stimulation, in contrast to other species, including the mouse and humans.

\section{References}

(Botts et al. 1994; Capen et al. 2001; Duprat et al. 1990; Faccini et al. 1990; Frith and Dunn 1994; Frith and Ward 1988b; Hamlin II and Banas 1990; Laroque et al. 1997; Longeart 1996; Nyska and Maronpot 1999; Patterson et al. 1995; Rosol et al. 2013; Strandberg 1996d; Tischler 1996; Tischler and Coupland 1994; Tischler et al. 1990)

\section{Hyperplasia, medullary, focal (H) Adrenal Gland}

(Figures 128, 129, and 130)

$$
\begin{aligned}
& \text { Species } \\
& \text { Mouse; Rat. }
\end{aligned}
$$

\author{
Synonyms \\ Nodular hyperplasia. \\ Pathogenesis/cell of origin \\ Adrenal medullary (chromaffin) cell.
}

Diagnostic Features

- Focal increase in number of chromaffin cells.

- Usually solitary, infrequently multifocal.

- No compression or minimal compression of medulla or cortex.

- Cellular architecture is maintained.

- Cells may be smaller or larger and have tinctorial differences of the cytoplasm.

- Nuclear-cytoplasmic ratio may be increased.

- Mitotic figures are rare.

- Lesion size is usually less than $50 \%$ of normal size of the medulla when the entire medulla is in the plane of sectioning.

\section{Differential Diagnosis}

Pheochromocytoma, Benign:

- Distinct compression at the periphery is present.

- Architecture is not maintained.

- Typically larger than $50 \%$ of the normal medulla.

\section{Comment}

Medullary hyperplasia is much more common in rats compared to mice, and the incidence is strain dependent. Strains with a high incidence include the Wistar, Gunn, Long-Evans, Fisher 344, and Sprague-Dawley. Multiple, separate adjacent foci of hyperplasia are not considered a neoplasm, even if their total size is larger than $50 \%$ of the normal medulla. Size of the foci of hyperplasia are considered a weak criterion for differentiation from adenoma even when the sections are through the center of the adrenal gland. Cells in medullary hyperplasia can consist of both epinephrine (adrenalin) and norepinephrine (noradrenalin), cells, whereas pheochromocytomas usually consist of norepinephrine cells. Rat adrenal medullary cells readily respond to mitogenic stimulation, in contrast to other species, including mouse and man.

\section{References}

(Botts et al. 1994; Capen et al. 2001; Duprat et al. 1990; Faccini et al. 1990; Frith and Dunn 1994; Frith and Ward 1988b; Hamlin II and Banas 1990; Laroque et al. 1997; Longeart 1996; Nyska and Maronpot 1999; Patterson et al. 1995; Rosol et al. 2013; Strandberg 1996d; Tischler 1996; Tischler and Coupland 1994; Tischler et al. 1990)

\section{Pheochromocytoma, benign (B) Adrenal Gland}

(Figures 131 and 132)

$$
\begin{aligned}
& \text { Species } \\
& \text { Mouse; Rat. }
\end{aligned}
$$


Synonym

Tumor, medullary, benign, pheochromocytoma type.

Pathogenesis/cell of origin

Adrenal medullary (chromaffin) cell.

Diagnostic Features

- Mass located in the adrenal medulla, may extend into the cortex.

- Single or multiple, unilateral or bilateral in rats. Usually unilateral in the mouse.

- May replace the entire medulla and cortex.

- Compression of the normal cortex or medulla at the tumor periphery.

- Cells organized in nests, rows, and cords.

- Often contains dilated blood vessels.

- Cells are large or small and the smaller cells have greater cytoplasmic basophilia.

- Cellular atypia may be present.

- Hemorrhage and necrosis may be present.

- Mitotic figures are absent or infrequent.

- Usually larger than $50 \%$ of the normal medulla.

\section{Differential Diagnoses}

Hyperplasia, Medullary, Focal:

- No or minimal compression of the adjacent tissues.

- Normal architecture is maintained.

- Usually smaller than half of the normal medulla.

Hyperplasia, Medullary, Diffuse:

- No or minimal compression of the adjacent tissues.

- Normal architecture is maintained.

- Entire medulla affected.

Pheochromocytoma, Complex, Benign:

- Contains well differentiated ganglion cells in addition to pheochromocytoma cells.

Pheochromocytoma, Malignant:

- Invasive growth or distant metastasis.

- Mitotic figures may be numerous.

Adenoma, Cortical:

- Presence of cytoplasmic lipid droplets.

- Eosinophilic cytoplasm.

- Negative immunohistochemical reaction for chromaffin cell biomarkers (see comment).

\section{Comment}

Adrenal medullary cells have a positive chromaffin reaction using histochemical stains (e.g., Cherukian-Schenck stain) and are immunohistochemically positive for tyrosine hydroxylase, chromogranin A, synaptophysin or neuronspecific enolase. Most pheochromocytomas in rats consist of norepinephrine cells and are negative for phenylethanolamine N-methyl transferase (PNMT), which is present in epinephrine cells. Pheochromocytoma cells contain dense core secretory granules by electron microscopy, which are absent in adrenal cortical adenomas.

\section{References}

(Botts et al. 1994; Capen et al. 2001; Duprat et al. 1990; Faccini et al. 1990; Frith and Dunn 1994; Frith and Ward 1988b; Hamlin II and Banas 1990; Hill et al. 2003; Laroque et al. 1997; Longeart 1996; Molenaar et al. 1990; Nyska and Maronpot 1999; Pace et al. 2002; Patterson et al. 1995; Rosol et al. 2013; Russfield 1967; Strandberg 1996d; Tischler 1996; Tischler et al. 1990; Unger et al. 1990; Wright et al. 1990)

\section{Pheochromocytoma, malignant (M) Adrenal Gland}

(Figures 133, 134, and 135)

Species

Mouse; Rat.

Synonym

Tumor, medullary, malignant, pheochromocytoma type.

Pathogenesis/cell of origin

Adrenal medullary (chromaffin) cell.

\section{Diagnostic Features}

- Invasive growth into the adrenal cortex or through the adrenal capsule, vascular invasion, or distant metastasis to the lungs, liver, lymph nodes, bone marrow or other organs.

- Cells organized in nests, rows and cords often with distended blood vessels.

- Cells are large or small and the smaller cells have greater cytoplasmic basophilia.

- Cellular atypia may be present.

- Hemorrhage and necrosis may be present.

- Mitotic figures may be numerous.

\section{Differential Diagnoses}

Pheochromocytoma, Benign:

- No evidence of invasive growth or distant metastases.

Carcinoma, Cortical:

- Presence of cytoplasmic lipid droplets.

- Eosinophilic cytoplasm.

Pheochromocytoma, Complex, Malignant:

- Contains well differentiated ganglion cells in addition to pheochromocytoma cells.

Neuroblastoma, Malignant:

- Composed of small basophilic cells.

- Cells palisade along blood vessels and stroma.

- Cells form circular, rosette-like structures.

- Nuclei can be round, oval, or elongated and are intensely basophilic.

- Neurofibrillary cytoplasmic extensions may be observed.

- Composed predominantly ( $>80 \%$ ) of neuroblasts.

- Ganglion cells may be present. 


\section{Comment}

Adrenal medullary cells have a positive chromaffin reaction using histochemical stains (e.g., Cherukian-Schenck stain) and are immunohistochemically positive for tyrosine hydroxylase, chromogranin A, synaptophysin or neuron-specific enolase. Chromaffin cells in the normal adrenal gland may be found in small nests in the adrenal cortex, especially near the hilar venules and should not be interpreted as invasion. In addition, small numbers of chromaffin cells may be present within sinusoids that likely represent an artifact of tissue preparation and must be differentiated from vascular invasion. In some cases, malignant pheochromocytomas invade large vessels, such as the vena cava or aorta, forming large intravascular tumors and thrombi that partially occlude the vessels.

\section{References}

(Aguzzi et al. 1990; Botts et al. 1994; Capen et al. 2001; Duprat et al. 1990; Faccini et al. 1990; Frith 1996a; Frith and Dunn 1994; Frith and Ward 1988b; Hamlin II and Banas 1990; Hill et al. 2003; Laroque et al. 1997; Longeart 1996; Maita et al. 1988; Majeed and Harling 1986; Nyska and Maronpot 1999; Pace et al. 2002; Rosol et al. 2013; Russfield 1967; Strandberg 1996d; Unger et al. 1990; Wright et al. 1990)

\section{Pheochromocytoma, complex, benign (B) Adrenal Gland}

(Figure 136)

\section{Species}

Mouse; Rat.

\section{Synonyms}

Complex pheochromocytoma, benign; tumor, medullary, benign, complex pheochromocytoma type.

\section{Pathogenesis/cell of origin}

Adrenal medullary (chromaffin) cell.

\section{Diagnostic Features}

- Contains morphological components of both pheochromocytoma and ganglioneuroma in which neither component predominates (i.e., neither component represents $>$ $80 \%$ of the mass).

- Cell types include well differentiated ganglion cells with eosinophilic nerve fibers and chromaffin cells. Chromaffin cells may be variably sized, with scant basophilic cytoplasm, and may be intermingled with nerve fibers or form distinct areas.

- Located in the adrenal medulla, and may extend into the cortex

- Distinct compression at the tumor periphery.

- Cellular atypia may be present.

- Mitotic figures may be absent or infrequent.

- Usually larger than $50 \%$ of the normal thickness of the medulla.

\section{Differential Diagnoses}

Hyperplasia, Medullary, Focal:

- No or minimal compression of the adjacent parenchyma.

- Architecture is maintained.

- Not larger than $50 \%$ of the normal medulla.

Pheochromocytoma, Complex, Malignant:

- Invasive growth and/or distant metastasis.

- Usually only the pheochromocytoma component develops malignant features.

Pheochromocytoma, Malignant or Pheochromocytoma, Benign:

- Tumors are composed predominantly $(>80 \%)$ of chromaffin cells.

Ganglioneuroma, Benign:

- Tumor is composed of well differentiated ganglion cells, Schwann cells, satellite cells and neurofibrils.

\section{Comment}

Adrenal medullary cells have a positive chromaffin reaction using histochemical stains (e.g., Cherukian-Schenck stain) and are immunohistochemically positive for tyrosine hydroxylase, chromogranin A, synaptophysin or neuron-specific enolase.

\section{References}

(Botts et al. 1994; Capen et al. 2001; Duprat et al. 1990; Faccini et al. 1990; Frith 1996a; Frith and Dunn 1994; Frith and Ward 1988b; Glaister et al. 1977; Hamlin II and Banas 1990; Laroque et al. 1997; Longeart 1996; Martinez and Mog 2001; Molenaar et al. 1990; Nyska and Maronpot 1999; Patterson et al. 1995; Reznik and Germann 1996a; Rosol et al. 2013; Russfield 1967; Strandberg 1996d; Wright et al. 1990)

\section{Pheochromocytoma, complex, malignant (M) Adrenal Gland}

Species

Mouse; Rat.

Synonyms

Ganglioneuroblastoma; Tumor, medullary, malignant, complex pheochromocytoma type.

Pathogenesis/cell of origin

Adrenal medullary (chromaffin) cell.

\section{Diagnostic Features}

- Invasion into the cortex or through the adrenal capsule; vascular invasion.

- Chromaffin cells may metastasize to distant sites.

- Cell types include ganglion cells with eosinophilic nerve fibers and malignant chromaffin cells that may be intermingled or form distinct areas.

- Malignant chromaffin cells are usually small with scant 
basophilic cytoplasm.

- Cells are arranged in nests, rows and cords surrounding numerous, often distended, blood vessels.

- Cellular atypia may be present.

- Mitotic figures may be numerous.

\section{Differential Diagnoses}

Pheochromocytoma, Complex, Benign:

- No evidence of invasive growth or distant metastasis.

Pheochromocytoma, Malignant:

- Neoplasms are composed predominantly $(>80 \%)$ of chromaffin cells.

Neuroblastoma, Malignant:

- Lack of or few differentiated ganglion cells or other components of mature nervous tissue.

Carcinoma, Cortical:

- Carcinoma cells of the cortex often contain lipid droplets.

- Negative immunohistochemistry for chromaffin cell biomarkers.

\section{Comment}

There should be unequivocal evidence of invasion or metastasis for a malignant classification. For example, large adenomas can expand beyond the normal adrenal. Small groups of chromaffin cells can be present within the cortex or near the hilus area of the adrenal gland as a normal anatomical feature. In addition, small numbers of chromaffin cells may be present within sinusoids that likely represent an artifact of tissue preparation and must be differentiated from vascular invasion. In some cases, malignant tumors can invade large vessels, such as the vena cava or aorta, forming large intravascular tumors and thrombi that partially occlude the vessels.

Adrenal medullary cells have a positive chromaffin reaction using histochemical stains (e.g., Cherukian-Schenck stain) and are immunohistochemically positive for tyrosine hydroxylase, chromogranin A, synaptophysin or neuron-specific enolase.

\section{References}

(Aguzzi et al. 1990; Botts et al. 1994; Capen et al. 2001; Duprat et al. 1990; Faccini et al. 1990; Frith 1996a; Frith and Dunn 1994; Frith and Ward 1988b; Glaister et al. 1977; Hamlin II and Banas 1990; Laroque et al. 1997; Longeart 1996; Maita et al. 1988; Martinez and Mog 2001; Nyska and Maronpot 1999; Patterson et al. 1995; Reznik and Germann 1996a; Rosol et al. 2013; Russfield 1967; Strandberg 1996d; Wright et al. 1990)

Ganglioneuroma, benign (B) Adrenal Gland (Figure 137)

Species

Mouse; Rat.
Synonym

Tumor, medullary, benign, ganglioneuroma type.

Pathogenesis/cell of origin

Neural crest sympathetic neuroblast (sympathoblast).

Diagnostic Features

- Predominant portion $(>80 \%)$ of the tumor consists of large, well differentiated ganglion cells and nerve fibers. May also contain Schwann and satellite cells.

- Located in the adrenal medulla and may extend into the cortex.

- May replace the entire medulla.

- Distinct compression at the tumor periphery.

Differential Diagnoses

Pheochromocytoma, Complex, Benign or Pheochromocytoma, Complex, Malignant:

- Presence of neoplastic chromaffin cells $(<80 \%$ of the tumor) in addition to ganglion cells and nerve fibers.

- Adrenal, capsular or vascular invasion or distant metastasis (in the malignant variant).

Neuroblastoma, Malignant:

- Composed predominantly ( $>80 \%$ ) of small basophilic neuroblasts.

- Cells palisade along blood vessels and stroma.

- Cells form circular, rosette-like structures.

- Ganglion cells may be present.

Pheochromocytoma, Benign:

- Presence of neoplastic chromaffin cells $(>80 \%$ of the tumor).

\section{Comment}

Ganglioneuromas are rare in rats and very rare in laboratory mice.

\section{References}

(Botts et al. 1994; Capen et al. 2001; Duprat et al. 1990; Faccini et al. 1990; Frith 1996a; Frith and Dunn 1994; Frith and Ward 1988b; Glaister et al. 1977; Hamlin II and Banas 1990; Kiupel et al. 2008b; Laroque et al. 1997; Longeart 1996; Molenaar et al. 1990; Nyska and Maronpot 1999; Pace et al. 2002; Patterson et al. 1995; Reznik and Germann 1996a; Reznik et al. 1980; Rosol et al. 2013; Russfield 1967; Shirai et al. 2012; Tischler 1992; Tischler et al. 1990; Wright et al. 1990)

\section{Neuroblastoma, malignant (M) Adrenal Gland}

(Figures 138 and 139)

Species

Mouse; Rat.

Synonyms

Ganglioneuroblastoma; Sympathicoblastoma; Tumor, medullary, malignant. 


\section{Pathogenesis/cell of origin}

Neural crest sympathogonia or sympathoblast cells.

\section{Diagnostic Features}

- Composed predominantly (> 80\%) of small, basophilic neuroblasts.

- Cells palisade along blood vessels and stroma.

- Cells occasionally form circular, rosette-like structures.

- Usually a spherical tumor that originates in the medulla.

- May compress the medulla and cortex.

- Nuclei can be round, oval, or elongated and are intensely basophilic.

- Neurofibrillary cytoplasmic extensions may be observed.

- Ganglion cells may be present.

- Cellular atypia may be present.

- Mitotic figures may be numerous.

- May invade into the cortex, through the adrenal capsule and into surrounding tissues.

\section{Differential Diagnoses}

Pheochromocytoma, Malignant:

- Composed of chromaffin cells that do not form rosettelike structures.

Pheochromocytoma, Complex, Malignant:

- Contains differentiated ganglion cells and nerve fibers (> $20 \%$ ) in addition to malignant chromaffin cells.

\section{Comment}

Neuroblastomas are very rare tumors in laboratory rats. Distant metastasis has not been reported in spontaneous tumors. Spontaneous neuroblastomas are rare in mice, but are reported in transgenic mice. It was reported that transgenic mice with the polyoma virus middle $\mathrm{T}$ antigen linked to the thymidine kinase promoter developed neuroblastoma in multiple organs, including the adrenal glands, which expressed the $\mathrm{N}$-myc oncogene and metastasized (Aguzzi et al. 1990).

\section{References}

(Aguzzi et al. 1990; Asamoto et al. 2001; Botts et al. 1994; Capen et al. 2001; Duprat et al. 1990; Faccini et al. 1990; Frith 1996a; Frith and Dunn 1994; Frith and Ward 1988b; Hamlin II and Banas 1990; Laroque et al. 1997; Longeart 1996; Maita et al. 1988; Nyska and Maronpot 1999; Pace et al. 2002; Patterson et al. 1995; Reznik and Germann 1996b; Rosol et al. 2013; Russfield 1967; Strandberg 1996d; Warren et al. 1966; Wright et al. 1990)

\section{Myelolipoma (B) Adrenal Gland (Figure 140)}

\section{Species}

Mouse; Rat.

\section{Pathogenesis/cell of origin}

Uncertain; Mesenchymal stem cells.

\section{Diagnostic Features}

- Area of adipocytes in the cortex with a variable amount of hematopoietic cells resembling bone marrow.

- Osseous metaplasia may be present.

Differential Diagnoses

Osseous Metaplasia:

- Well differentiated lamellar bone in the cortex.

Extramedullary Hematopoiesis:

- Hematopoietic cells without adipocytes or bone.

\section{Comment}

This is a benign, tumor-like lesion that occurs in both young and old rats, and has been described in humans and other species.

\section{References}

(Botts et al. 1994; Jones et al. 1997; Kiupel et al. 2008b; Lloyd et al. 2004; Neville and O'Hare 1982; Selye and Stone 1950) 


\section{Endocrine PANCREAS: Islets of LANGERHANS}

\section{A. Nonproliferative Lesions}

Amyloid, islet (N) Pancreas, Endocrine (Figure 141)

Species

Mouse; Rat.

Synonyms

Amyloidosis; Amyloid deposition.

\section{Pathogenesis/cell of origin}

Degenerative change characterized by extracellular deposition of polypeptide fragments derived from immunoglobulin or serum proteins in $\beta$-pleated sheet conformation.

\section{Diagnostic Features}

- Diffuse interstitial (extracellular) amorphous pale eosinophilic material in the islets.

- Green birefringence using polarized light with positive Congo red stain.

- Positive with Thioflavine T stains or specific immunohistochemical antibodies.

\section{Differential Diagnosis}

Fibrosis:

- More fibrillary in appearance on H\&E sections.

- Congo Red and immunohistochemical staining negative.

- Positive collagen staining (van Gieson's, Picrosirius red and Masson-trichrome).

\section{Comment}

Two forms of spontaneously occurring amyloidosis have been described in rodents: AA and ApoA2. The incidence and severity of amyloid deposits in pancreatic islets may increase with age and can be seen usually with systemic amyloidosis in amyloidosis-prone mouse strains. Islet amyloid due to amylin deposition does not occur spontaneously in rats and mice as it occurs in cats, non-human primates, and humans. Certain transgenic strains have been developed to overexpress islet amyloid polypeptide (IAPP, amylin) in islets. Amyloidosis can be accompanied by islet cell degeneration and islet cell loss.

\section{References}

(Aigelsreiter et al. 2007; Frith et al. 2000; Greaves 2012b; Höppener et al. 1994; Inoue and Kisilevsky 1996; Johnson et al. 1992; Leiter and Herbert 1996; Majeed 1993; Solomon et al. 1999; Wong et al. 2008)

\section{Angiectasis, islet (N) Pancreas, Endocrine (Figure 142)}

Species

Mouse; Rat.
Synonyms

Hemangiectasis; Hemangiectasia; Teleangiectasis; Telangiectasia.

Pathogenesis/cell of origin

Vasculature of the islets.

Diagnostic Features

- Wide, blood-filled vessels lined by well differentiated endothelial cells.

- No distortion of islet architecture.

- If present, usually affects few islets.

- May progress to focal hemorrhage.

\section{Differential Diagnoses}

Congestion:

- Diffuse dilatation of blood-filled vessels not distorting the architecture of the affected tissue.

Hemorrhage:

- Extravascular blood present in the islet parenchyma.

- Chronic hemorrhage maybe associated with hemosiderin-laden macrophages.

\section{Comment}

Angiectasis is rare in normal islets of rats and mice. Angiectasis may occur in hyperplastic islets or islet cell neoplasms and does not warrant a separate diagnosis.

\section{References}

(Boorman and Sills 1999; Frith et al. 2000)

\section{Apoptosis, islet cell (N) Pancreas, Endocrine}

Species

Mouse; Rat.

Synonyms

Cell death.

\section{Pathogenesis/cell of origin}

Apoptosis is a regulated, energy-dependent process. There are three main pathways (extrinsic, intrinsic, and perforin/ granzyme pathway) that all involve activation of procaspases. All three pathways culminate in activation of caspase 3, which begins the execution pathway. With apoptotic cell death there is generally no cell rupture, no release of pro-inflammatory mediators, and thus no inflammation. The apoptotic bodies express phosphatidylserine on the cell surface, which is recognized by macrophages.

\section{Diagnostic Features}

- Condensed, hypereosinophilic cytoplasm.

- Chromatin condensation and nuclear pyknosis or karyorrhexis.

- Small, rounded cell blebs and fragments with intact cytoplasmic membrane (apoptotic bodies). 
- No inflammatory response.

- Phagocytosis of apoptotic cells by adjacent cells or macrophages.

\section{Differential Diagnosis}

Necrosis, Single Cell:

- Cells are often swollen and may be ruptured.

- No presence of apoptotic bodies.

- Inflammatory cell infiltration may be present.

\section{Comment}

Apoptosis is an uncommon event in the normal pancreatic islets and is often increased in diabetes mellitus. Islet cell apoptosis can contribute significantly to loss of $\beta$-cell mass in the late stages of diabetes mellitus in rats and mice leading to islet atrophy. Apoptotic cells can be identified by staining for TUNEL or activated effector caspases, such as caspase-3. If both apoptosis and single-cell necrosis are present, then a combined term is appropriate.

\section{References}

(Elmore 2007; Elmore et al. 2016; Frith et al. 2000; Galluzzi et al. 2012; Kroemer et al. 2009; Levin et al. 1999; Teta et al. 2005)

\section{Atrophy, islet cell (N) Pancreas, Endocrine}

(Figures 143 and 144)

Species

Mouse; Rat.

\section{Pathogenesis/cell of origin}

Follows persistent cell death, (e.g. such as apoptosis/necrosis in the islets). Most commonly $\beta$ cells are affected; however, if the cell type is known then appropriate modifiers include $\beta$ cell, $\alpha$ cell, $\delta$ cell, $\gamma$ cell, or $\varepsilon$ cell.

\section{Diagnostic Features}

- Islet cells may have decreased cytoplasm.

- Islet cells may be decreased in number.

- Islets may have an irregular shape and are reduced in size.

- Cells may have condensed, eosinophilic or pale cytoplasm.

- Relative increase in the number of islet cells of the nonaffected subtypes.

- $\alpha$ cells may be randomly distributed instead of normal peripheral distribution.

- Acinar cells may occur occasionally within atrophic islets.

\section{Differential Diagnosis}

Hypoplasia, Islet Cell:

- Present at birth or in neonatal animals.

- Normal peripheral distribution of $\alpha$ cells.
Degranulation, Islet Cell:

- Pale homogenous or condensed, eosinophilic cytoplasm.

- Cytoplasm may be reduced.

- Reduced immunohistochemical staining for specific endocrine hormones.

\section{Comment}

This is a rare change in aged rodents. It is important not to over-diagnose islet atrophy, because sectioning of a normal islet near its periphery would lead to a small crosssectional area. Definitive diagnosis of islet atrophy may require widespread lesions or the use of histomorphometry or stereology. Islet atrophy can be observed in older animals and animals treated with exogenous insulin. In diabetic animals it may result from persistent $\beta$-cell apoptosis or necrosis, and it can be widespread or diffuse, and may progress to islet fibrosis.

\section{References}

(Bartels et al. 2016; Boorman and Sills 1999; Frith et al. 2000; Greaves 2012b; Leiter and Herbert 1996; Yamaoka et al. 1998)

\section{Degranulation, islet cell (N) Pancreas, Endocrine}

Species

Mouse; Rat.

Synonym

Cytoplasmic pallor.

\section{Pathogenesis/cell of origin}

Degranulation of $\beta$ cells can be observed in situations of increased insulin demand such as metabolic syndrome and diabetes mellitus. Most commonly $\beta$ cells are affected; however, if the cell type is known then appropriate modifiers include $\beta$ cell, $\alpha$ cell, $\delta$ cell, $\gamma$ cell, or $\varepsilon$ cell.

\section{Diagnostic Features}

- Pale homogenous or condensed, eosinophilic cytoplasm.

- Cytoplasm may be reduced.

- Reduced staining of insulin-sensitive stains such as aldehyde fuchsin (in case of $\beta$ cells) or immunohistochemical staining for specific endocrine hormones.

\section{Differential Diagnosis}

Atrophy, Islet Cell:

- Islet cells may have decreased cytoplasm.

- Islet cells may be decreased in number.

- Islets may have an irregular shape and are reduced in size.

- Cells may have condensed, eosinophilic or pale cytoplasm.

- Relative increase in the number of islet cells of the nonaffected subtypes. 


\section{Comment}

Degranulation of $\beta$ cells is difficult to recognize in standard H\&E-stained slides. Immunohistochemistry is the method of choice to visualize change in cellular hormone content. Degranulation of $\beta$ cells may be followed by vacuolation if the stimulus for increased insulin secretion persists. Decreased insulin staining of pancreatic islets can also be a consequence of a reduced $\beta$-cell mass. After streptozotocin administration in rats, degranulation of $\beta$ cells is observed within 24 hours.

\section{References}

(Adeghate et al. 2010; Frith et al. 2000; Greaves 2012b; Mense and Rosol 2018a; Rosol et al. 2013)

Fibrosis, islet (N) Pancreas, Endocrine (Figures 145 and 146)

Species

Mouse; Rat.

\section{Pathogenesis/cell of origin}

Collagen deposition by fibroblasts following inflammation, necrosis, or hemorrhage.

\section{Diagnostic Features}

- Deposition of collagen by fibroblasts in and around pancreatic islets.

- Distribution can be focal (single islet), multifocal (multiple islets) or diffuse (all islets).

- Loss of endocrine cells of the islets.

- Decreased staining intensity for insulin.

- Positive for collagen stains which include Masson trichrome, van Gieson, Picrosirius red.

\section{Differential Diagnosis}

Amyloid:

- Diffuse interstitial (extracellular) amorphous pale eosinophilic material in the islets.

- Green birefringence using polarized light with positive Congo red stain.

- Positive with Thioflavine T stains, or specific immunohistochemical antibodies.

\section{Comment}

The pattern of fibrosis can vary between species and within different strains and individual animals. It is observed as an end-stage of islet atrophy in mice and rats with chronic diabetes mellitus. Fibrosis can rarely be observed spontaneously in old rats and mice and can be found together with chronic inflammation and changes of the surrounding exocrine pancreas (e.g., atrophy). Spontaneous hemorrhage and fibrosis of islets and peri-islet pancreatic tissue occurs in aging Sprague-Dawley rats, particularly males.

\section{References}

(Frith et al. 2000; Greaves 2012b; Imaoka et al. 2009; Imaoka et al. 2007; Leiter and Herbert 1996; Riley et al. 1990a)

\section{Hemorrhage, islet (N) Pancreas, Endocrine}

Species

Mouse; Rat.

Pathogenesis/cell of origin

Vasculature of islet of Langerhans; vascular injury or hemorrhagic diathesis.

Diagnostic Features

- Free erythrocytes in the extravascular space often focused at the islet-acinar interface and may extend into the adjacent exocrine tissue.

- Chronic hemorrhage usually is accompanied by hemosiderin-laden macrophages.

- Cholesterol clefts and fibrosis may also occur.

\section{Differential Diagnoses}

Angiectasis:

- Blood present within dilated blood vessels.

\section{Comment}

Iron stains (such as Prussian blue) may be useful in confirming the presence of hemosiderin at sites of chronic hemorrhage. Spontaneous hemorrhage and fibrosis of islets and peri-islet pancreatic tissue occurs in aging SpragueDawley rats, particularly males.

\section{References}

(Imaoka et al. 2009; Imaoka et al. 2007)

\section{Hypertrophy, islet cell (N) Pancreas, Endocrine}

Species

Mouse; Rat.

Pathogenesis/cell of origin

Endocrine cells of the islets of Langerhans.

\section{Diagnostic Features}

- Increased cytoplasmic area of endocrine cells.

- Cytoplasm is pale and may contain small vacuoles.

- Islet cell subtypes can be identified using immunohistochemistry for hormones, such as insulin and glucagon.

- May occur simultaneously with hyperplasia.

\section{Differential Diagnosis}

Hyperplasia, Islet Cell:

- Islet cells are increased in number.

- Islets are increased in size.

\section{Comment}

Hypertrophy of islet cells can be seen in cells with increased production and secretion of endocrine hormones. The endocrine cells can be identified using immunohistochemistry for their respective hormones, such as insulin or glucagon. When hypertrophy occurs with hyperplasia, it is not necessary to have a separate diagnosis for hypertrophy 
or the combined diagnosis of hypertrophy/hyperplasia can be used.

\section{References}

(Moroki et al. 2013; Yamazaki et al. 2009)

\section{Infiltrate, inflammatory cell (N) Pancreas, Endocrine}

Species

Mouse; Rat.

\section{Pathogenesis/cell of origin}

Accumulation of small number of inflammatory cells; uncertain pathogenesis or local immune response.

\section{Diagnostic Features}

- Small numbers of inflammatory cells within the islet.

- Usually consists of mononuclear cells.

- May be focal (single islet) or multifocal (multiple islets).

- No tissue damage or vascular changes.

\section{Differential Diagnosis}

Inflammation:

- Usually more extensive and typically associated with tissue damage and/or vascular changes (such as congestion).

Lymphoma:

- Islets are infiltrated and replaced by neoplastic lymphoid cells.

- Other parenchymal and lymphoid organs (e.g., spleen and lymph nodes) are frequently involved.

\section{References}

(Boorman and Sills 1999; Frith et al. 2000; Greaves 2012b)

Inflammation (N) Pancreas, Endocrine (Figure 146)

\section{Species}

Mouse; Rat.

\section{Synonyms}

Insulitis; Islitis; Isletitis.

\section{Pathogenesis/cell of origin}

Inflammatory change.

\section{Diagnostic Features}

- Infiltration of mononuclear cells including lymphocytes, macrophages, and plasma cells. Neutrophils may occasionally be present. Usually affects many islets.

- Islet cells may have degenerative changes.

- May be associated with tissue damage or vascular changes (such as congestion).

- Apoptosis or necrosis of islet cells may be present.
Differential Diagnosis

Infiltrate, Inflammatory Cell:

- Usually small number of inflammatory cells, most commonly mononuclear cells, in few islets.

- No tissue damage.

Lymphoma:

- Islets are infiltrated and replaced by neoplastic lymphoid cells.

- Other parenchymal and lymphoid organs (e.g., spleen and lymph nodes) are frequently involved.

\section{Comment}

Insulitis has been described in the BB Wistar rat and nonobese diabetic mouse. Both animals are used as models of juvenile-onset diabetes mellitus (Type 1). Juvenile-onset diabetes mellitus often has insulitis early in the disease and likely represents immune-mediated inflammation. Animals with adult onset (Type 2 diabetes mellitus) may have increased number of macrophages in the islets that contribute to degeneration of islet cells due to the release of inflammatory cytokines.

References

(Chou et al. 2013; Ehses et al. 2007; Frith et al. 2000; Greaves 2012b; Mense and Rosol 2018a; Rosol et al. 2013)

\section{Metaplasia, hepatocyte (N) Pancreas, Endocrine}

(Figure 147)

Species

Mouse; Rat.

\section{Pathogenesis/cell of origin}

Metaplasia of peri-islet exocrine cells to hepatocyte-like cells.

\section{Diagnostic Features}

- Presence of hepatocyte-like cells at the periphery of the islet.

- Most commonly one or several layers in the periphery of the islet, but may appear as a focal nest or occupy the entire islet.

- May involve single or multiple islets.

\section{Comment}

'Pancreatic hepatocytes' can occur spontaneously and can be induced by certain chemicals notably peroxisome proliferating agents, a methyl-group-deficient diet, and by a copper depletion/repletion.

\section{References}

(Armocida et al. 1994; Frith et al. 2000; Greaves 2012b; Riley et al. 1990b; Yamakawa et al. 1995) 
Necrosis, single cell (N) Pancreas, Endocrine

(Figure 148)

Species

Mouse; Rat.

\section{Synonyms}

Single cell necrosis; Cell death; Oncotic cell death; Oncotic necrosis.

\section{Pathogenesis/cell of origin}

Necrosis is unregulated, energy-independent, passive cell death. The mechanism of necrotic cell death involves development of disturbances in membrane function and integrity, calcium dysregulation, and the inability to reverse mitochondrial dysfunction. Necrotic cells leak their cytoplasmic contents into the surrounding interstitium, which provokes an inflammatory reaction.

\section{Diagnostic Features}

- Swollen, pale eosinophilic islet cells.

- Karyorrhexis, karyolysis or pyknosis of nuclei.

- Vacuolation can be observed.

- Can be focal (single islet), multifocal (multiple islets), and diffuse (all islets).

- Inflammatory cell infiltration may be present.

- Single cells necrosis can occur with apoptosis in the same islet.

\section{Differential Diagnosis}

Apoptosis, Islet Cell:

- Condensed, hypereosinophilic cytoplasm.

- Chromatin condensation and nuclear pyknosis or karyorrhexis.

- Small, rounded cell blebs and fragments with intact cytoplasmic membrane (apoptotic bodies).

- No inflammatory response.

\section{Comment}

Necrosis can be induced by certain chemicals, including streptozotocin, alloxan, and cyclosporine A. Early events in chemically-induced islet cell necrosis include marked vacuolar change, swelling, and degranulation of the islet cells. Single cell necrosis is rarely observed spontaneously in rats and mice due to the slow turnover of endocrine cells in the pancreatic islets. It is observed in diabetic mice and rats and $\beta$ cells are predominantly affected. If both necrosis and apoptosis occur in the islets, a combined term is appropriate.

\section{References}

(Chandra et al. 2013; Elmore et al. 2016; Galluzzi et al. 2012; Kroemer et al. 2009)

Pigment, islet (N) Pancreas, Endocrine (Figure 149)

Species

Mouse; Rat.

\section{Pathogenesis/cell of origin}

Accumulation of colored products derived from cellular or erythrocyte (hemoglobin) breakdown or lipid peroxidation of cellular membranes.

\section{Diagnostic Features}

- Multiple types of pigment, such as iron or lipofuscin, can accumulate in the islet cells or between the islet cells.

- Brown granular pigment is the most commonly seen.

- Pigments may stain positive with PAS and occasionally acid-fast stain.

- Iron will stain positive with Prussian blue.

\section{Comment}

Brown pigments have been observed in older rats and mice in endocrine cells of the pancreatic islets. Hemosiderin and lipofuscin are the most common pigments. Hemosiderinladen macrophages represent a remnant of previous hemorrhage. Lipofuscin (derived from cell membranes) can be an indicator of increased cell turnover or death.

References

(Boorman and Sills 1999; Frith et al. 2000; Greaves 2012b; Imaoka et al. 2009; Imaoka et al. 2007)

Vacuolation, islet cell (N) Pancreas, Endocrine (Figure 150)

Species

Mouse; Rat.

Pathogenesis/cell of origin

Degeneration of $\beta$ cells.

Diagnostic Features

- Large intracytoplasmic vacuoles.

- Vacuoles are usually clear with H\&E stain.

- Vacuoles may contain glycogen, which is PAS-positive and amylase-sensitive.

\section{Comment}

Vacuolar changes can be observed in chemically induced toxic damage (such as with calcineurin inhibitors) or diabetes mellitus. Some agents that produce diabetes experimentally (e.g. streptozotocin, cyproheptadine and cyclizine) as well as compounds with diverse pharmacologic actions can cause diffuse vacuolar change of the $\beta$ cells. Vacuolation may also be a nonspecific finding unrelated to chemical exposure, representing glycogen accumulation, spontaneous hydropic degeneration during development of diabetes mellitus, or even a fixation artifact.

\section{References}

(Chandra et al. 2013; Frith et al. 2000; Gopinath and Mowat 2014; Greaves 2012b; Mense and Rosol 2018a; Nugent et al. 2008; Rosol et al. 2013) 


\section{B. Proliferative Lesions}

Hyperplasia, islet cell (H) Pancreas, Endocrine (Figure 151) Species

Mouse; Rat.

\section{Modifiers}

Elongated or irregular islet type; Focal (single islet); Multifocal (several islets); Diffuse (most islets).

\section{Pathogenesis/cell of origin}

Cells of islets of Langerhans, usually $\alpha$ or $\beta$ cells.

\section{Diagnostic Features}

- Hyperplastic islets are increased in size with increased islet cell numbers.

- Hyperplasia is typically multifocal or diffuse and does not affect all islets equally.

- The outline of hyperplastic islets may be round or irregular.

- The proportion of $\alpha$ and $\beta$ cells (or other islet cell types) may differ from normal.

- Coalescence of two or more smaller islets may occur.

- There is no compression of surrounding acinar tissue and no capsule is present.

- Islet cells are typically polygonal and in some cases elongated or hypertrophic.

- There are no tinctorial differences when compared with normal islet cells.

- Low mitotic activity and no cellular atypia.

- Scant fibrovascular stroma may separate groups of cells.

- Macrophages and inflammatory cells are rarely present within hyperplastic islets.

- Dilated vascular spaces and/or central cystic cavities may be present in hyperplastic islets of mice.

- Some islets may be elongated in shape.

\section{Differential Diagnosis}

Adenoma, Islet Cell:

- Compression of peripheral tissue and/or encapsulation.

- Growth pattern differs from hyperplastic islets with formation of sheets, nests, cords, or ribbons of neoplastic islet cells.

- Neoplastic cells may be larger or smaller, or darker or paler than hyperplastic islet cells.

\section{Comment}

Hyperplasia of either $\alpha$ and $\beta$ cells will disrupt the normal ratio and pattern of the islet cells. $\beta$ cells are normally located centrally in the islets and are surrounded by a small $\alpha$-cell margin. The incidence of hyperplastic islets is higher in male than female rats, as well as in rats fed ad libitum. Morphometry and statistical analysis of size and shape of the islets can be used to set the threshold for the diagnosis of hyperplasia. Size of the islets has been used as a diagnostic criterion. For example, islets with a diameter between 350 and $700 \mu \mathrm{m}$ were diagnosed as focal hyperplasia and islets with a diameter larger than $700 \mu \mathrm{m}$ diameter were diagnosed as adenoma. However, size of individual islets should not be used as the sole diagnostic feature for adenoma.

\section{References}

(Boorman and Sills 1999; Botts et al. 1994; Capen et al. 2001; Frith and Sheldon 1996; Frith and Ward 1988b; Greaves 2012b; Heath 1996c; Koivisto et al. 2012; Longnecker and Millar 1990; Mense and Rosol 2018a; Reaven and Reaven 1981; Rosol et al. 2013; Steiner et al. 2010; Stromberg et al. 1983; Zwicker and Eyster 1993)

\section{Adenoma, islet cell (B) Pancreas, Endocrine}

(Figures 152 and 153)

\section{Species}

Mouse; Rat.

\section{Synonyms}

Adenoma, endocrine pancreas; Tumor, islet cell, benign; Insulinoma, benign.

\section{Pathogenesis/cell of origin}

Cells of the islets of Langerhans, usually $\beta$ cells.

\section{Diagnostic Features}

- A well-circumscribed nodule that compresses adjacent acinar tissue.

- Variable encapsulation and a few tumor cells may be found in the capsule.

- Tumor growth occurs by expansion, but focal projections into the surrounding exocrine pancreatic tissue may occur in non-encapsulated tumors.

- Several growth patterns may be found including sheets, nests, cords, and ribbons, and occasionally palisading along small blood vessels.

- Cytoplasm of the tumor cells is usually pale or slightly eosinophilic.

- Tumor cells are well differentiated and generally uniform in size but variations may occur especially in the mouse.

- Karyomegaly may occur.

- Mitotic figures are uncommon.

- Small groups of acinar cells can be atrophied, but occasionally hypertrophic cells may be found within the tumor.

- Fibrous stroma is variable.

- Vasculature may be prominent.

- Vascular ectasia or hemorrhages may be present.

- Invasion into surrounding tissues and metastases are not present.

\section{Differential Diagnosis}

Hyperplasia, Islet Cell:

- No compression of acinar tissue and no encapsulation. 
- Typical islet architecture is maintained.

- Vascularity is not increased but vascular ectasia can occur.

- Distribution is usually multifocal.

Carcinoma, Islet Cell:

- Local invasion of capsule and acinar tissue often with proliferative fibrovascular stroma.

- Metastasis may be present.

- Cellular anaplasia, cellular and nuclear pleomorphism and/or, mitotic figures are common.

\section{Comment}

Spontaneous islet cell adenomas are rare in mice.

Most of the islet cell tumors produce insulin as demonstrated by immunohistochemical staining, but hypoglycemia does not occur in rats or mice in contrast to that common complication of insulinoma in dogs and humans. Islet cells containing somatostatin, glucagon or pancreatic polypeptide are usually randomly scattered throughout the neoplasm (as demonstrated by immunohistochemistry).

Size of the islets has been used as a diagnostic criterion in the past. Islets with a diameter between 350 and 700 $\mu \mathrm{m}$ were diagnosed as focal hyperplasia and islets with a diameter larger than $700 \mu \mathrm{m}$ were diagnosed as adenoma. However, the size of individual islets should not be used as the sole diagnostic feature for adenoma.

Knockout mice in which the Menl gene was disrupted specifically in pancreatic $\beta$-cells developed multiple islet cell adenomas at 6 months of age (Bertolino et al. 2003).

\section{References}

(Bertolino et al. 2003; Boorman and Sills 1999; Botts et al. 1994; Capen et al. 2001; Crabtree et al. 2001; Crabtree et al. 2003; Dillberger 1994; Frith and Sheldon 1996; Frith and Ward 1988a; Greaves 2012b; Heath 1996c; Koivisto et al. 2012; Longnecker and Millar 1990; Mense and Rosol 2018a; Reaven and Reaven 1981; Rosol et al. 2013; Spencer et al. 1986; Stromberg et al. 1983)

\section{Adenoma, acinar-islet cell (B) Pancreas, Endocrine}

(Figures 154 and 155)

Species

Mouse; Rat.

\section{Synonyms}

Mixed acinar-islet cell tumor, benign; Mixed pancreatic tumor, benign.

\section{Pathogenesis/cell of origin}

Multipotential cells of pancreas with differentiation into endocrine, acinar and ductal cells.

\section{Diagnostic Features}

- Well circumscribed solitary nodule that compresses adjacent tissue.
- Neoplastic cell population consists of both endocrine and exocrine cells, sometimes in nearly equal numbers.

- Encapsulation may be present.

- Tumor growth by expansion, usually with distinct margins.

- Both cell types may show mild atypia and increased mitotic activity.

- Atypia is often more pronounced in the acinar cells.

Differential Diagnosis

Adenoma, Islet Cell:

- There are no or few incorporated exocrine cells in the neoplasm.

- Incorporated exocrine cells have no cellular atypia, but occasionally are hypertrophic or atrophied.

\section{References}

(Boorman and Sills 1999; Botts et al. 1994; Heath 1996c; Mense and Rosol 2018a; Riley et al. 1990a; Riley et al. 1990b; Rosol et al. 2013)

\section{Carcinoma, islet cell (M) Pancreas, Endocrine}

(Figures 156 and 157)

Species

Mouse; Rat.

Synonyms

Adenocarcinoma, endocrine pancreas; Tumor, islet cell, malignant; Insulinoma, malignant.

Pathogenesis/cell of origin

Cells of islets of Langerhans, usually $\beta$ cells.

Diagnostic Features

- May be grossly visible as a tan to red nodule.

- Local invasion of capsule or adjacent acinar tissue, often with proliferative fibrovascular stroma.

- Cells vary from well-differentiated to pleomorphic and anaplastic.

- Growth pattern includes sheets, nests, cords, and ribbons.

- Fibrous capsule may be present.

- Cytoplasm is pale to eosinophilic with vesicular nuclei and prominent nucleoli.

- Mitotic figures may be numerous.

- Metastasis may occur, especially to the liver.

Differential Diagnosis

Adenoma, Islet Cell:

- No local invasion or metastasis.

- Tumor cells are generally well differentiated.

- Mitotic figures are uncommon.

\section{Comment}

Spontaneous islet cell carcinomas are rare in mice; however, histological features of malignancy are usually more 
pronounced in mice than in rats.

Most of the islet cell tumors produce insulin as demonstrated by immunohistochemical staining, but hypoglycemia does not occur in rats or mice in contrast to that common complication of insulinoma in dogs and humans.

\section{References}

(Boorman and Sills 1999; Botts et al. 1994; Capen et al. 2001; Dillberger 1994; Frith and Sheldon 1996; Frith and Ward 1988a; Greaves 2012b; Heath 1996c; Imazawa et al. 2001; Koivisto et al. 2012; Longnecker and Millar 1990; Mense and Rosol 2018a; Reaven and Reaven 1981; Rosol et al. 2013; Spencer et al. 1986; Stromberg et al. 1983)

\section{Carcinoma, acinar-islet cell (M) Pancreas, Endocrine}

\section{Species}

Mouse; Rat.

\section{Synonyms}

Mixed exocrine-endocrine carcinoma.

\section{Pathogenesis/cell of origin}

Multipotential cells of pancreas with differentiation into endocrine, acinar and ductal cells.

\section{Diagnostic Features}

- Neoplastic cell population consists of both endocrine and exocrine cells, sometimes in nearly equal numbers.

- Encapsulation may be present.

- Local invasion of capsule by the endocrine cells

- Both cell types may show atypia and increased mitotic activity.

- Atypia is often more pronounced in the acinar cells.

- Metastasis may occur, especially to liver.

\section{Differential Diagnosis}

Adenoma, Islet Cell:

- No local invasion or metastasis.

- Mitotic figures are uncommon.

- The number of exocrine cells is low.

- Incorporated groups of exocrine cells have no cellular atypia, but occasionally are hypertrophic or atrophied.

Adenoma, Acinar-Islet Cell:

- Local invasion or metastasis are not present

\section{Comment}

This tumor is rare; only one case has been documented in the rat.

\section{References}

(Boorman and Eustis 1990; Boorman and Sills 1999; Botts et al. 1994; Capella et al. 2004; Heath 1996c; Riley et al. 1990a; Riley et al. 1990b)

\section{ACKNOWLEDGEMENTS}

Photographs were kindly provided by the team members and Drs. Jonathan Carter (Covance, Harrogate, UK), Christopher Gray (Covance, Harrogate, UK), Michael Elwell (Covance, Chantilly, VA, USA), Kathrine Heider (EPC, Switzerland), Ron Herbert (National Institute of Environmental Health and Safety, Raleigh, NC, USA), Hijiri Iwata (LunaPath LLC, Hamamatsu, Japan), Virginie Piccicuto (Covance, Harrogate, UK), Graham Ince (Covance, Harrogate, UK), Claudio Petterino (Charles River, Edinburgh, UK), and Jerrold Ward (Global VetPathology, Montgomery Village, MD, USA). 


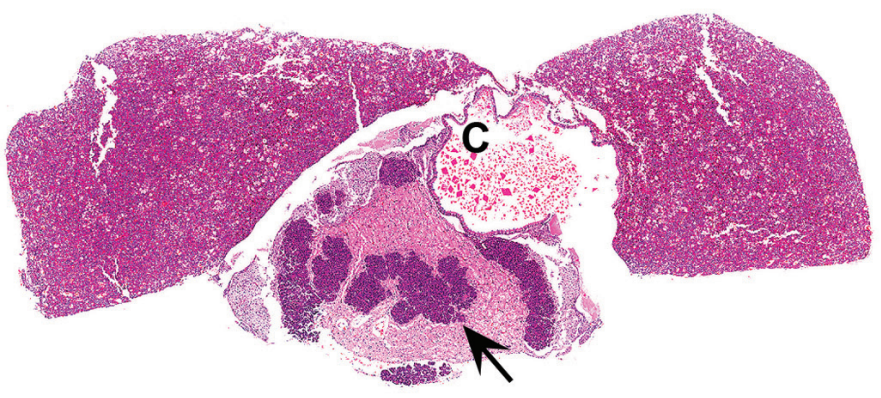

1
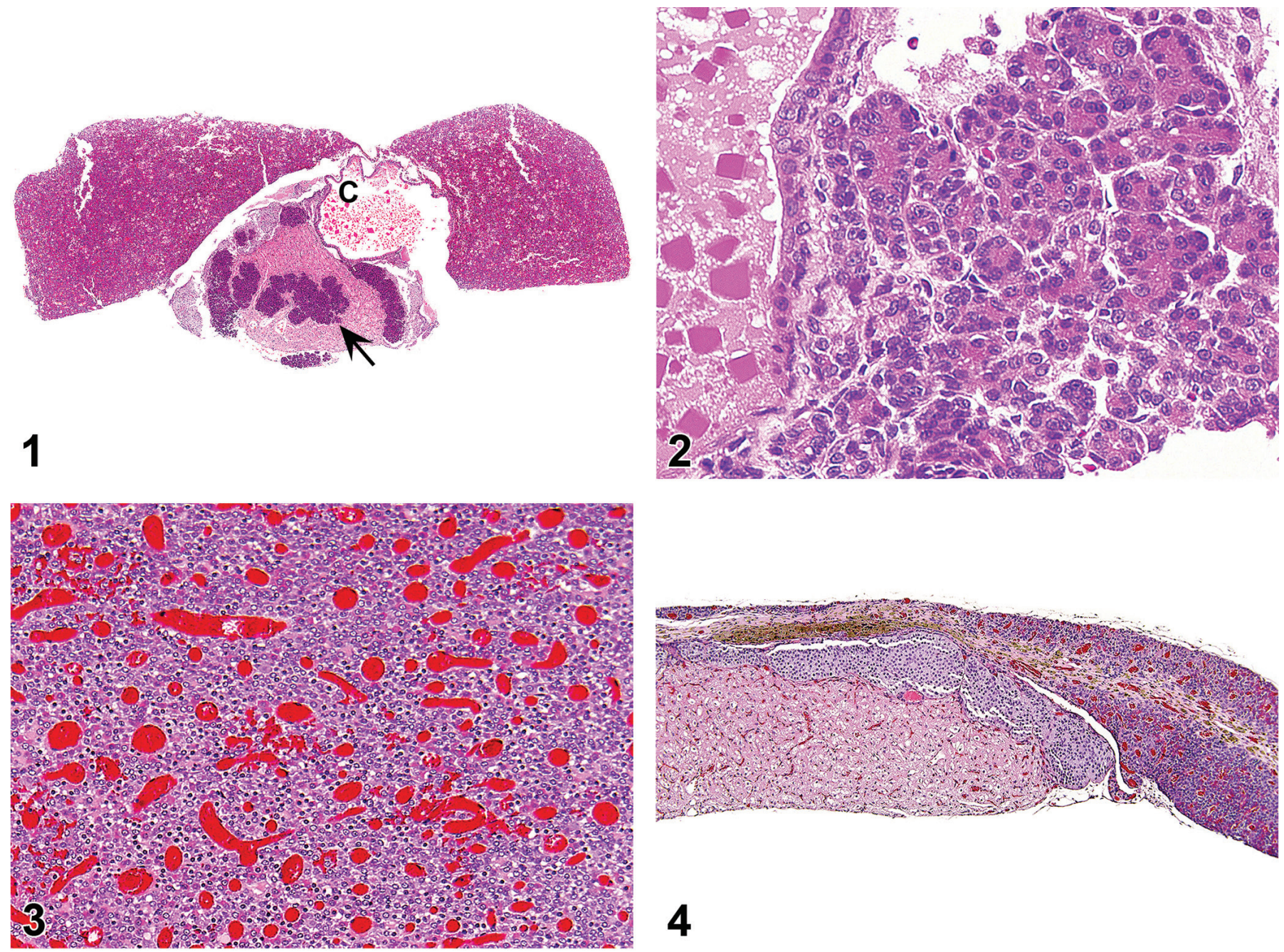

\section{4}
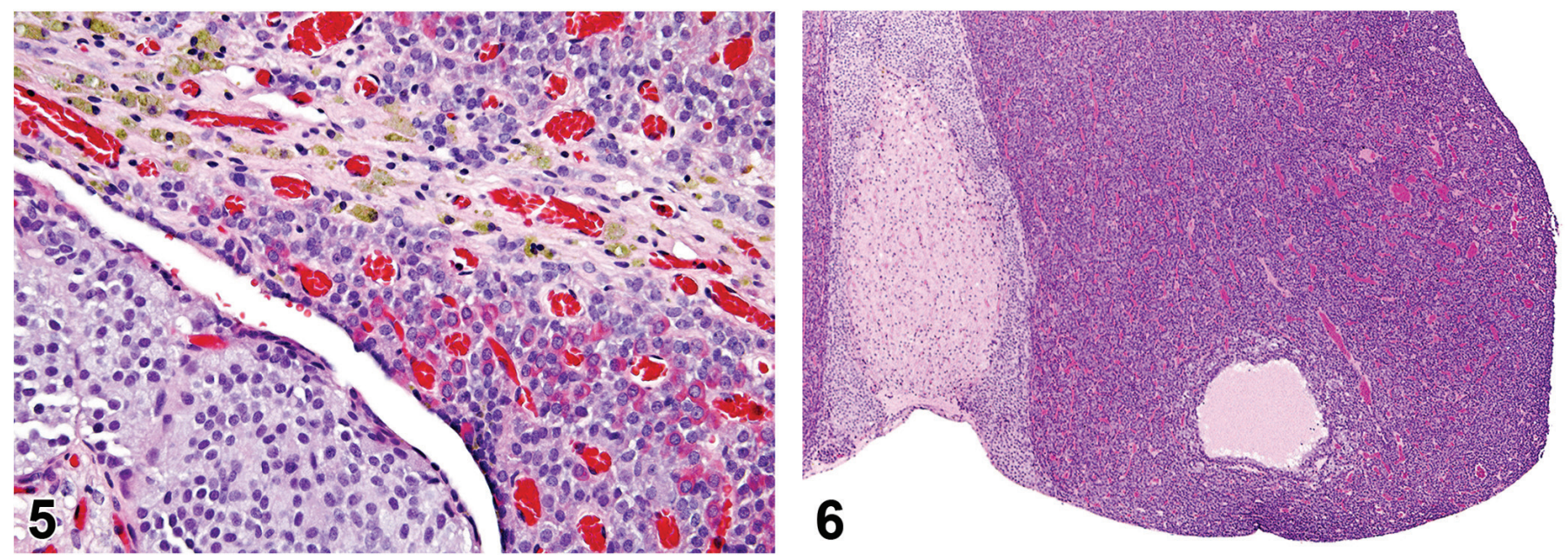

FIGURE 1._ Rat pituitary gland. Aberrant craniopharyngeal structures between the pars intermedia and pars nervosa and within the pars intermedia (arrow). Adjacent cyst of Rathke's cleft (C).

FigURE 2.- Rat pituitary gland. Aberrant craniopharyngeal structures with salivary gland-like differentiation and associated cyst of Rathke's cleft (left).

FIGURE 3.—Rat pituitary gland. Angiectasis of capillaries in the pars distalis.

Figure 4. - Rat pituitary gland. Atrophy of the pars distalis (top and right) with fibrosis and hemosiderophages. The pars intermedia and nervosa (lower and left) are normal.

FIGURE 5. - Rat pituitary gland. Atrophy of the pars distalis (upper right) with fibrosis, hemosiderophages, and mild angiectasis. The pars intermedia (lower left) is normal.

Figure 6. - Rat pituitary gland. Cyst in the pars distalis. 

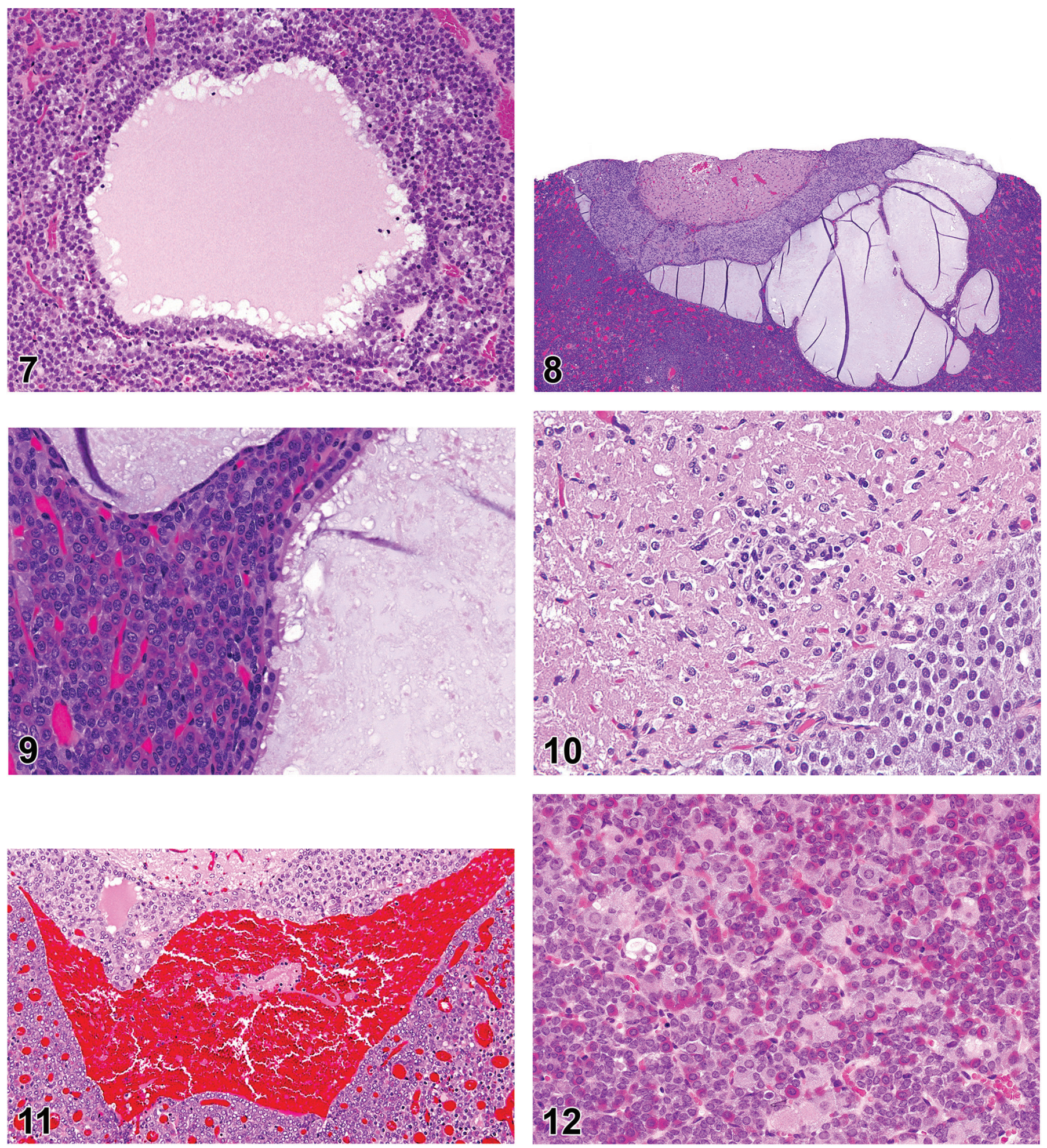

FIGURE 7.—-Rat pituitary gland. Cyst in the pars distalis lined by endocrine and folliculostellate cells.

Figure 8. - Rat pituitary gland. Cyst of Rathke's pouch, multiloculated.

FIGURE 9.— Rat pituitary gland. Cyst of Rathke's pouch. The lining epithelial cells are cuboidal or flattened. The cuboidal epithelial cells have luminal cilia.

Figure 10.- Rat pituitary gland. Gliosis, pars nervosa. Increased numbers of glial cells in the pars nervosa. The pars intermedia is on the lower right. Figure 11. - Rat pituitary gland. Hemorrhage into Rathke's pouch. Angiectasis in the pars distalis (lower left and right). Note the normal follicle present in the pars intermedia (upper left) formed by protein secretion from folliculostellate cells.

FIGURE 12.- Rat pituitary gland. Hypertrophy of chromophobic endocrine cells in the pars distalis. 

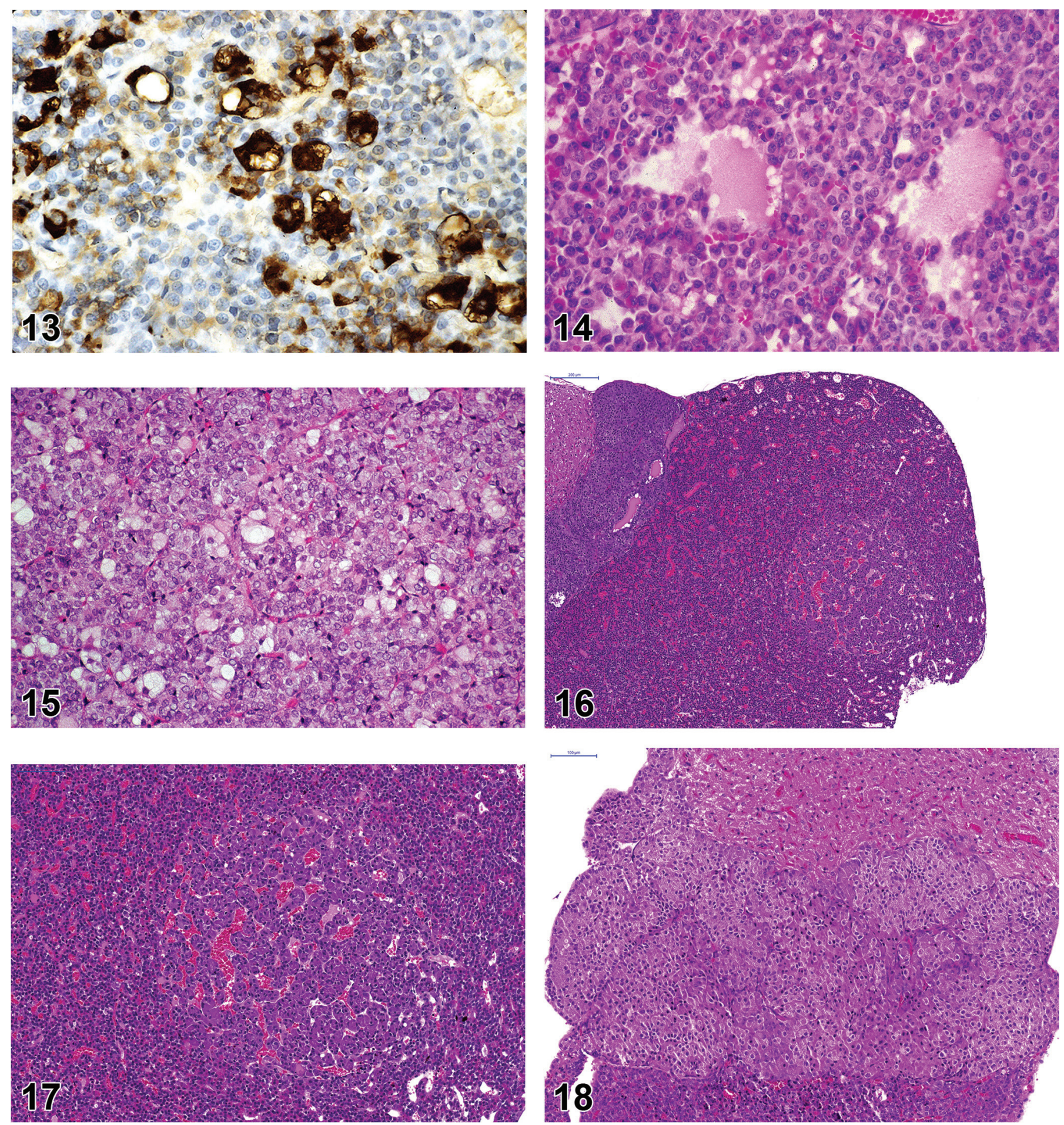

Figure 13.- Mouse pituitary gland. Hypertrophy of TSH cells with few cytoplasmic vacuoles in the pars distalis. Immunohistochemistry for TSH.

FIGURE 14.- Mouse pituitary gland. Pseudocyst formation in the pars distalis.

FIGURE 15.- Rat pituitary gland. Vacuolation of hypertrophied endocrine cells in the pars distalis.

Figure 16.- Rat pituitary gland. Hyperplasia, focal, pars distalis.

FiguRE 17.- Rat pituitary gland. Hyperplasia, focal, pars distalis.

Figure 18.- Mouse pituitary gland. Hyperplasia, diffuse, pars intermedia. 

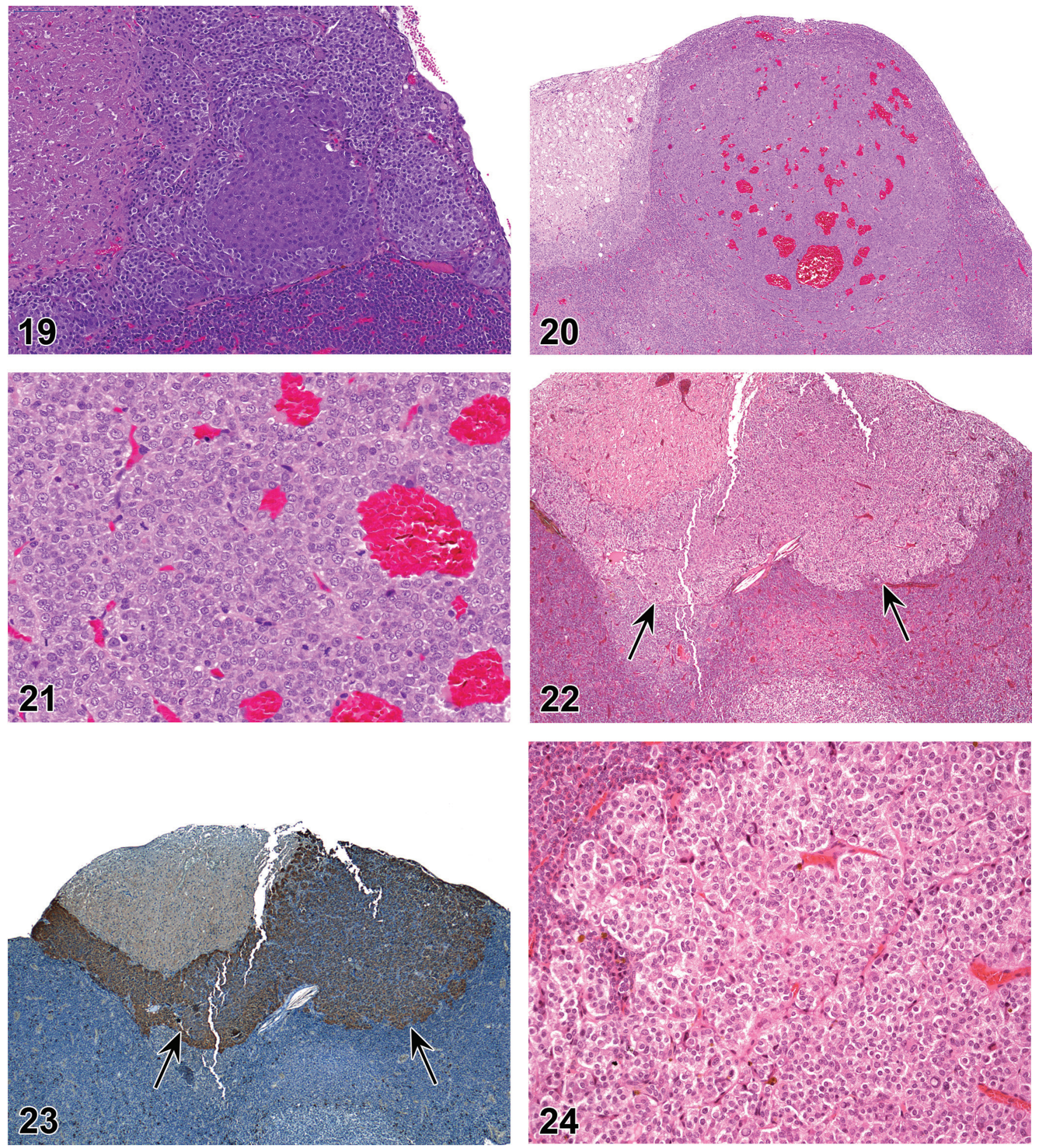

FIGURE 19.- - Rat pituitary gland. Hyperplasia, focal, pars intermedia.

Figure 20.- Rat pituitary gland. Adenoma, pars distalis.

Figure 21.-Rat pituitary gland. Adenoma, pars distalis.

FIGURE 22.- Rat pituitary gland. Adenoma, pars intermedia (arrows).

FIgURE 23.- Rat pituitary gland. Adenoma, pars intermedia (arrows). The pars intermedia and adenoma stain positive using ACTH immunohistochemistry (brown cytoplasmic staining). The adenoma is less positively stained.

FIGURE 24.- Rat pituitary gland. Adenoma, pars intermedia. The adenoma cells are enlarged. 

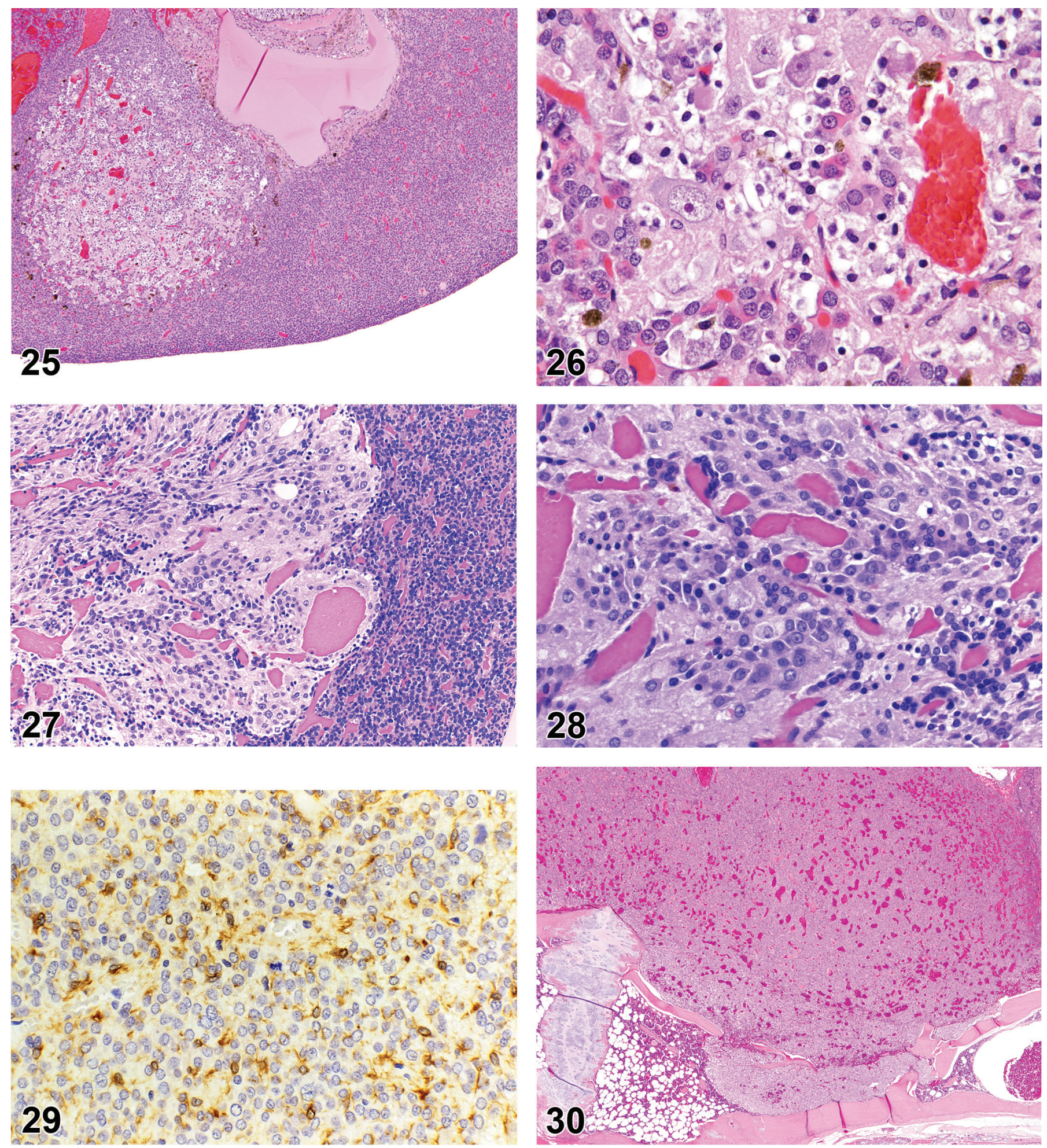

FIGURE 25.- Rat pituitary gland. Ganglioneuroma, pars distalis with hemosiderin pigment and a cyst of Rathke's pouch.

Figure 26.- Rat pituitary gland. Ganglioneuroma, pars distalis. Note the large ganglion cells.

Figure 27.- Rat pituitary gland. Pituicytoma, pars nervosa.

Figure 28.- Rat pituitary gland. Pituicytoma, pars nervosa.

FIGURE 29.- Rat pituitary gland. Pituicytoma, pars nervosa. Positive immunohistochemical staining for glial fibrillary acidic protein (GFAP), brown intracytoplasmic staining.

FIGURE 30.- Rat pituitary gland. Carcinoma, pars distalis with invasion into the sphenoid bone. 

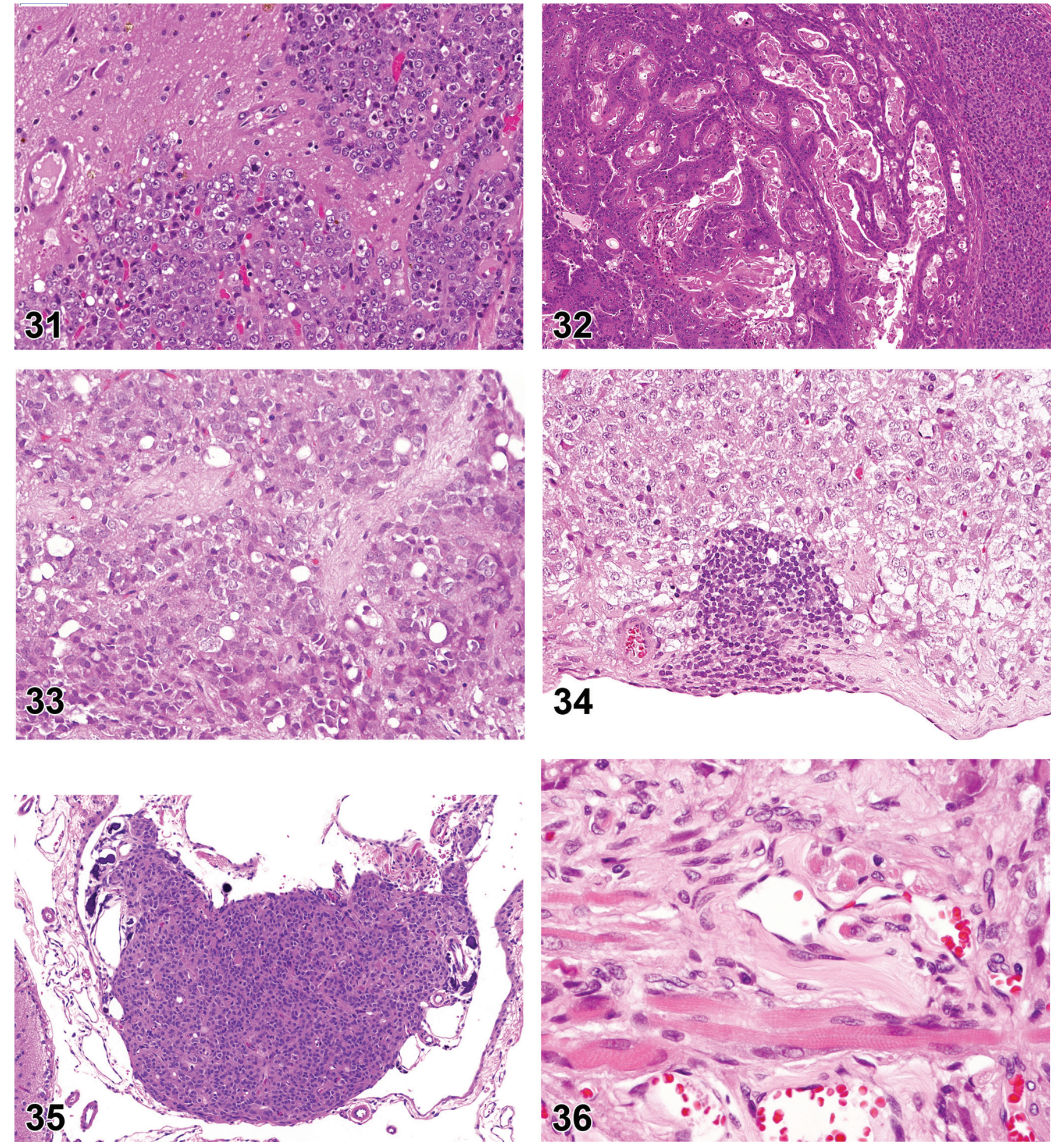

FIGURE 31.-Rat pituitary gland. Carcinoma, pars distalis with invasion into the neurophil of the brain.

FiguRE 32.-Rat pituitary gland. Craniopharyngioma with compression of the pars distalis.

Figure 33.-Rat pineal gland. Fibrosis.

FIGURE 34.-Rat pineal gland. Infiltrate, inflammatory cell.

Figure 35.- Rat pineal gland. Mineralization.

Figure 36.- Rat pineal gland. Striated muscle fibers. 

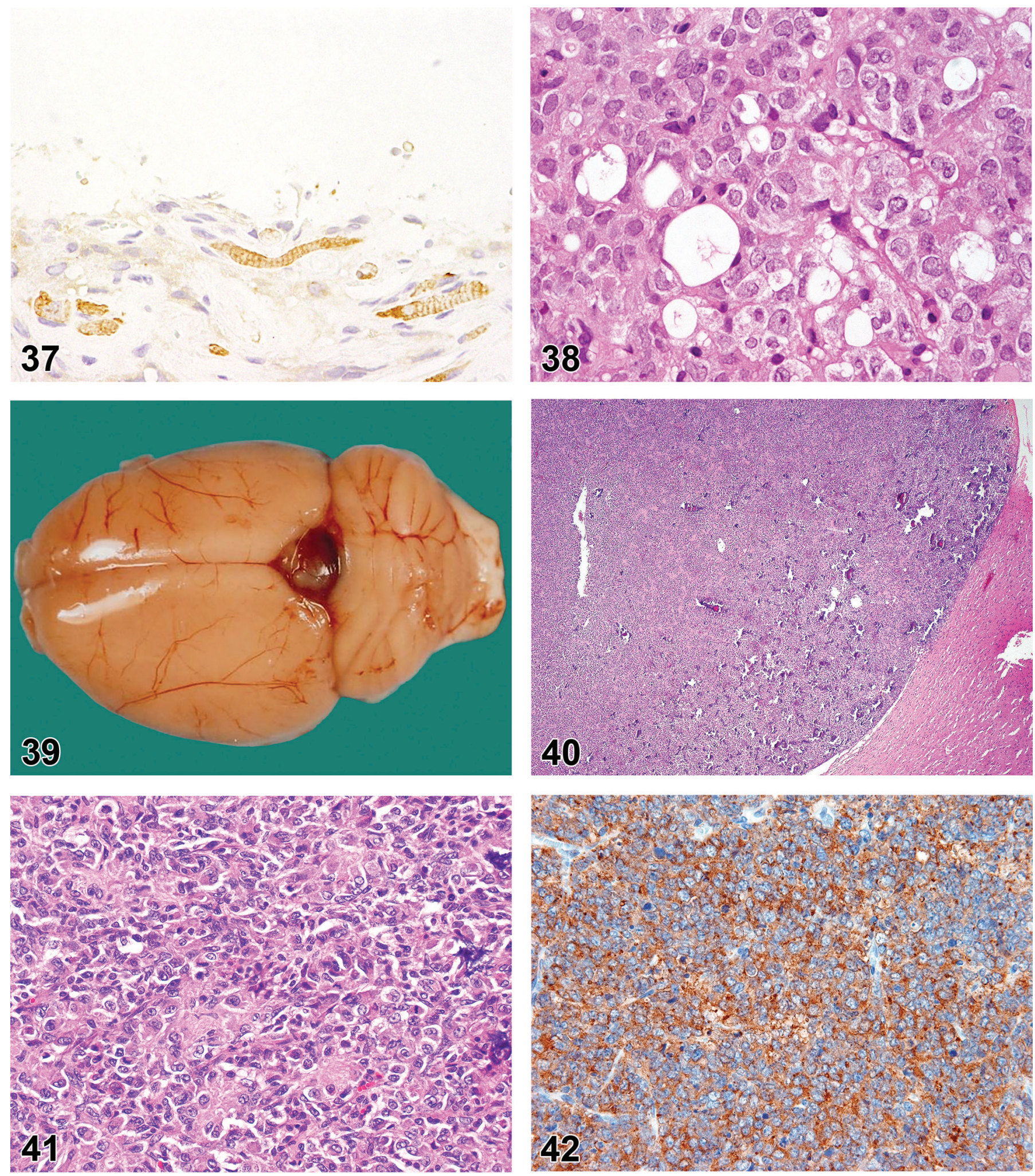

Figure 37.- Rat pineal gland. Striated muscle fibers. Positive immunohistochemical staining for desmin (brown cytoplasmic staining).

Figure 38.- Rat pineal gland. Vacuolation.

FIGURE 39. - Rat pineal gland. Pinealoma, benign.

Figure 40.- Rat pineal gland. Pinealoma, benign.

Figure 41.-Rat pineal gland. Pinealoma, benign.

Figure 42.-Rat pineal gland. Pinealoma, benign. Positive immunohistochemical staining for synaptophysin. 


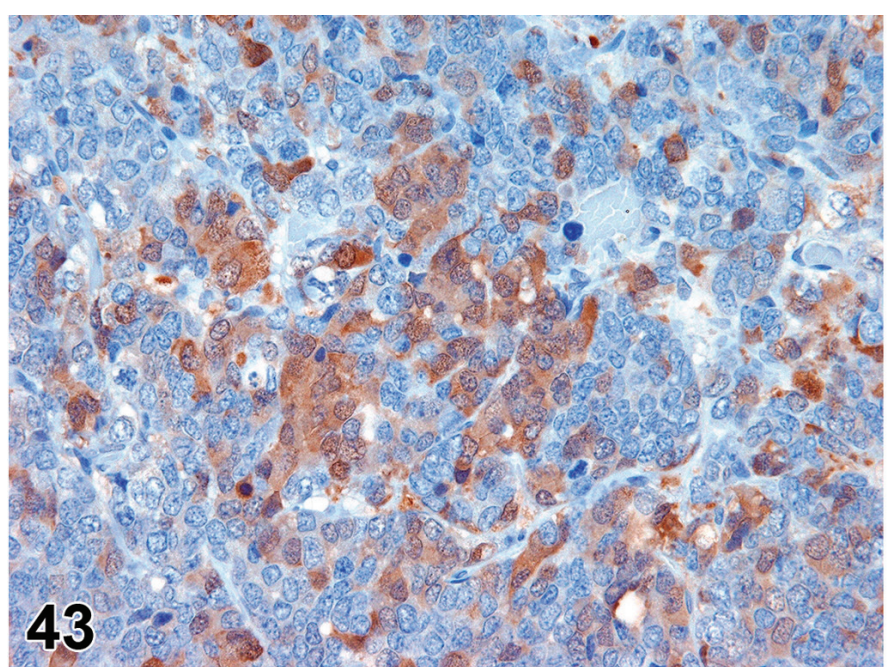

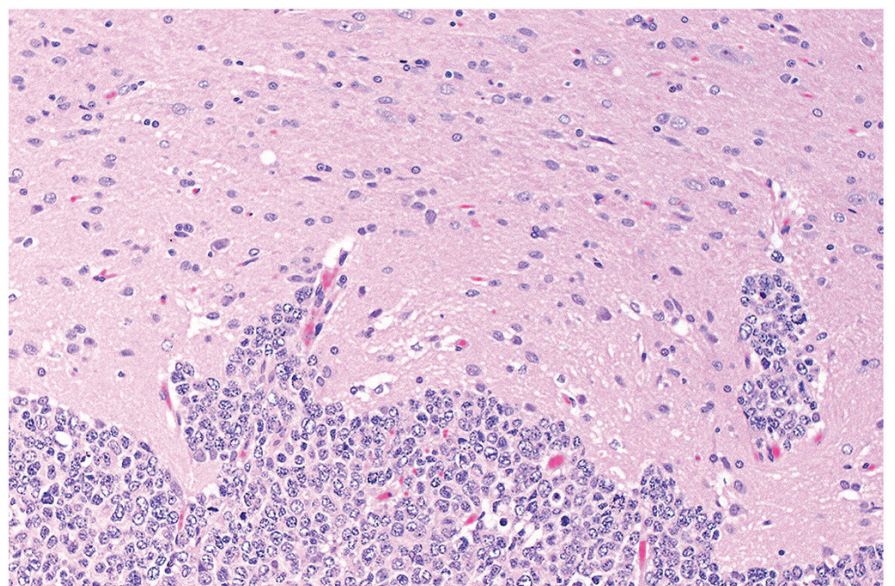

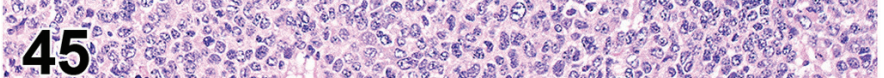

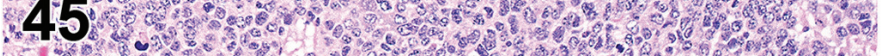

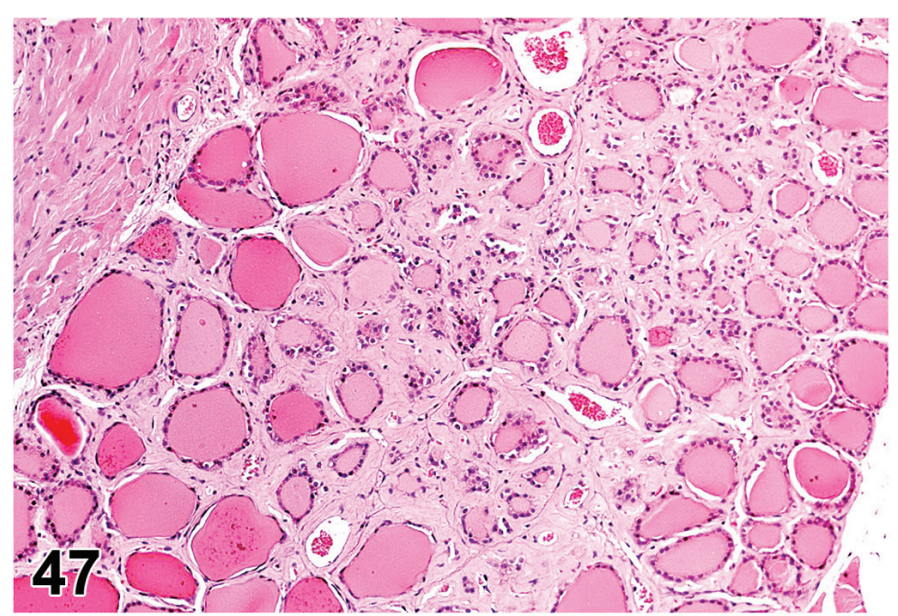

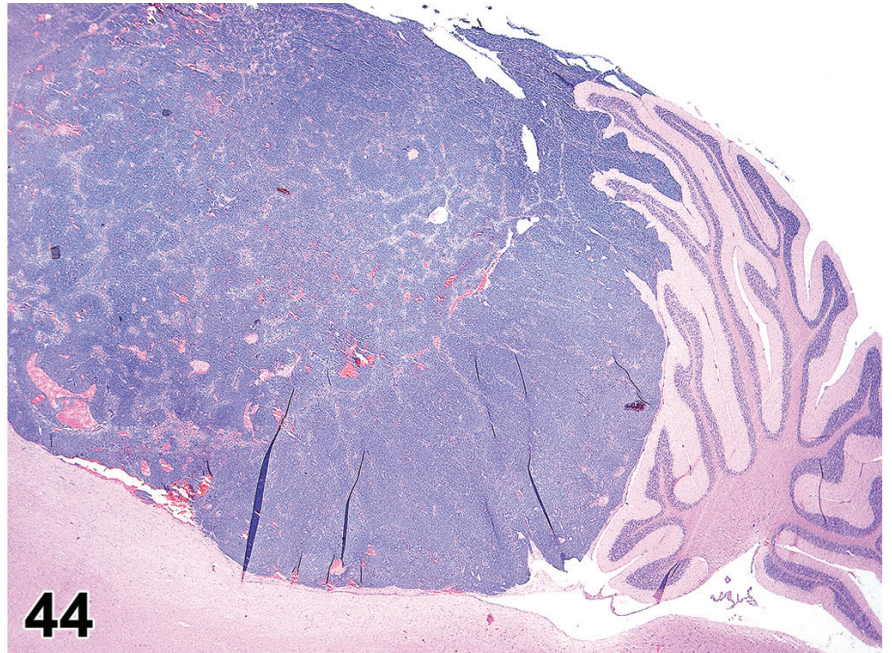
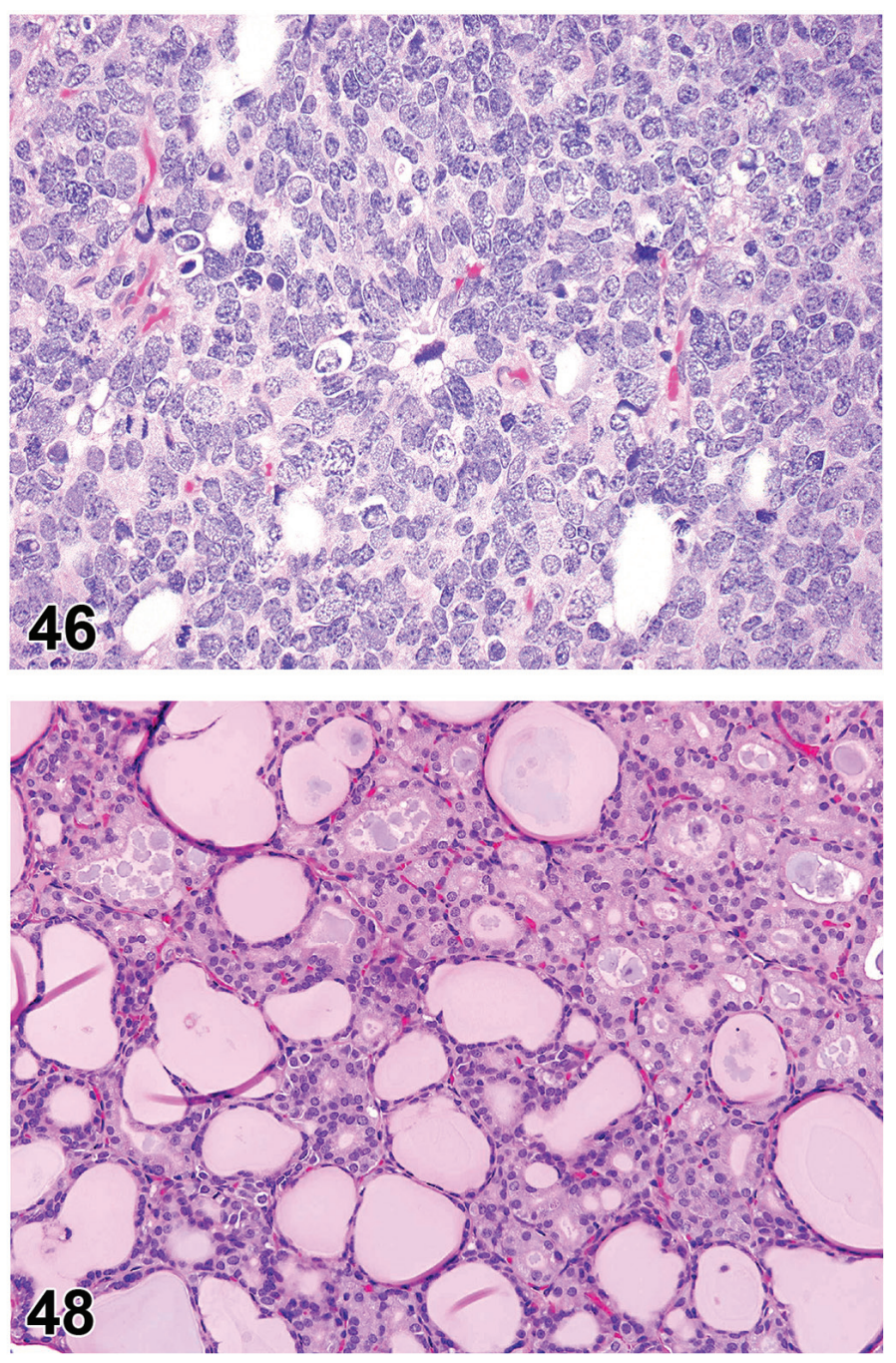

Figure 43.-Rat pineal gland. Pinealoma, benign. Positive immunohistochemical staining for neuron-specific enolase.

Figure 44.- Rat pineal gland. Pinealoma, malignant.

Figure 45.- Rat pineal gland. Pinealoma, malignant.

Figure 46. - Rat pineal gland. Pinealoma, malignant with pseudorosettes.

Figure 47.- Mouse thyroid gland. Amyloid.

Figure 48.- Rat thyroid gland. Colloid alteration.

FIGURE 49.- Mouse thyroid gland. Cystic follicle. 

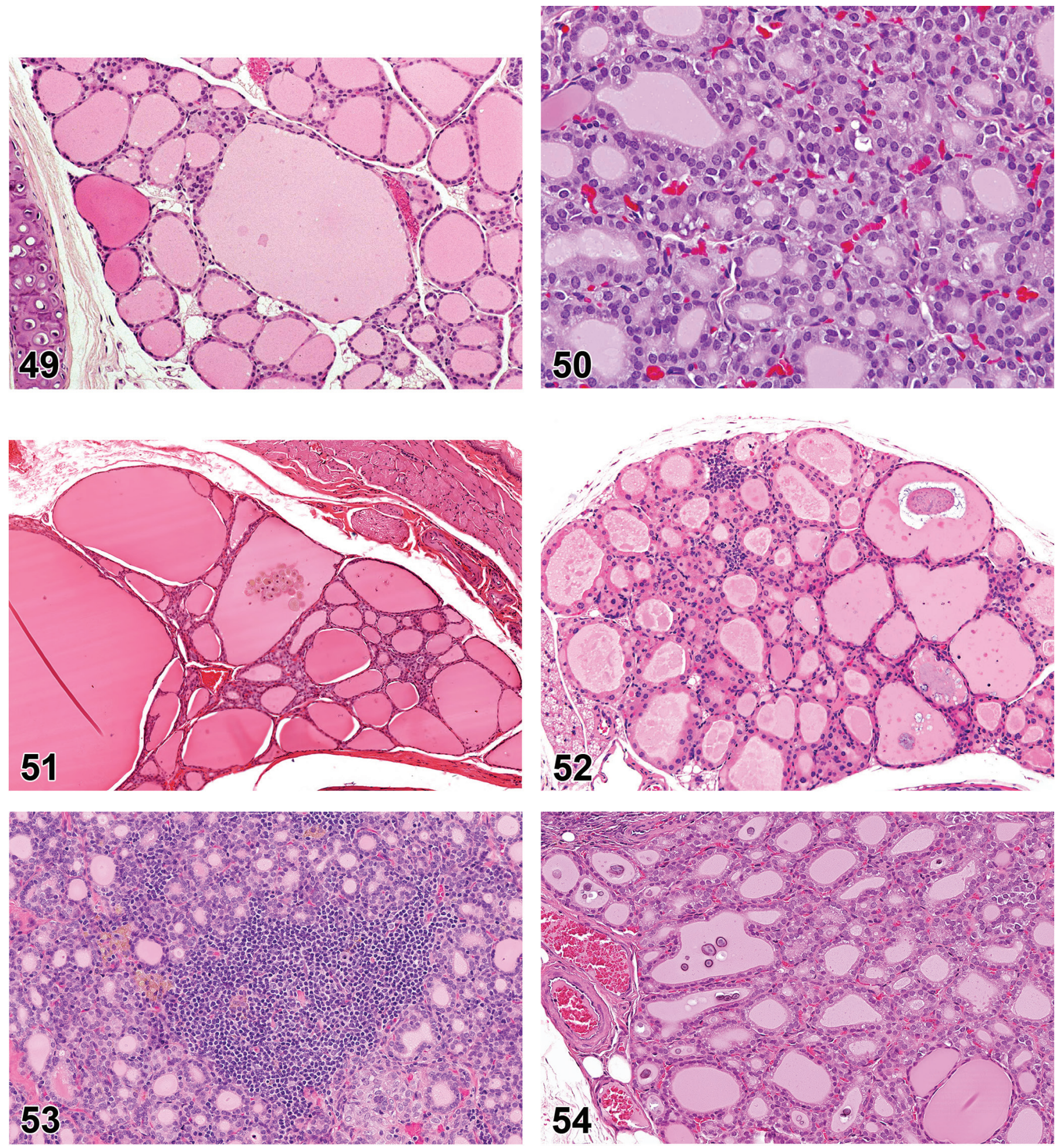

Figure 50.-Rat thyroid gland. Follicular cell hypertrophy.

Figure 51. - Rat thyroid gland. Follicular dilatation, diffuse.

FIGURE 52.-Mouse thyroid gland. Infiltrate, inflammatory cell.

Figure 53.- Rat thyroid gland. Inflammation, mononuclear.

FIGURE 54.-Rat thyroid gland. Mineralization of the colloid. 

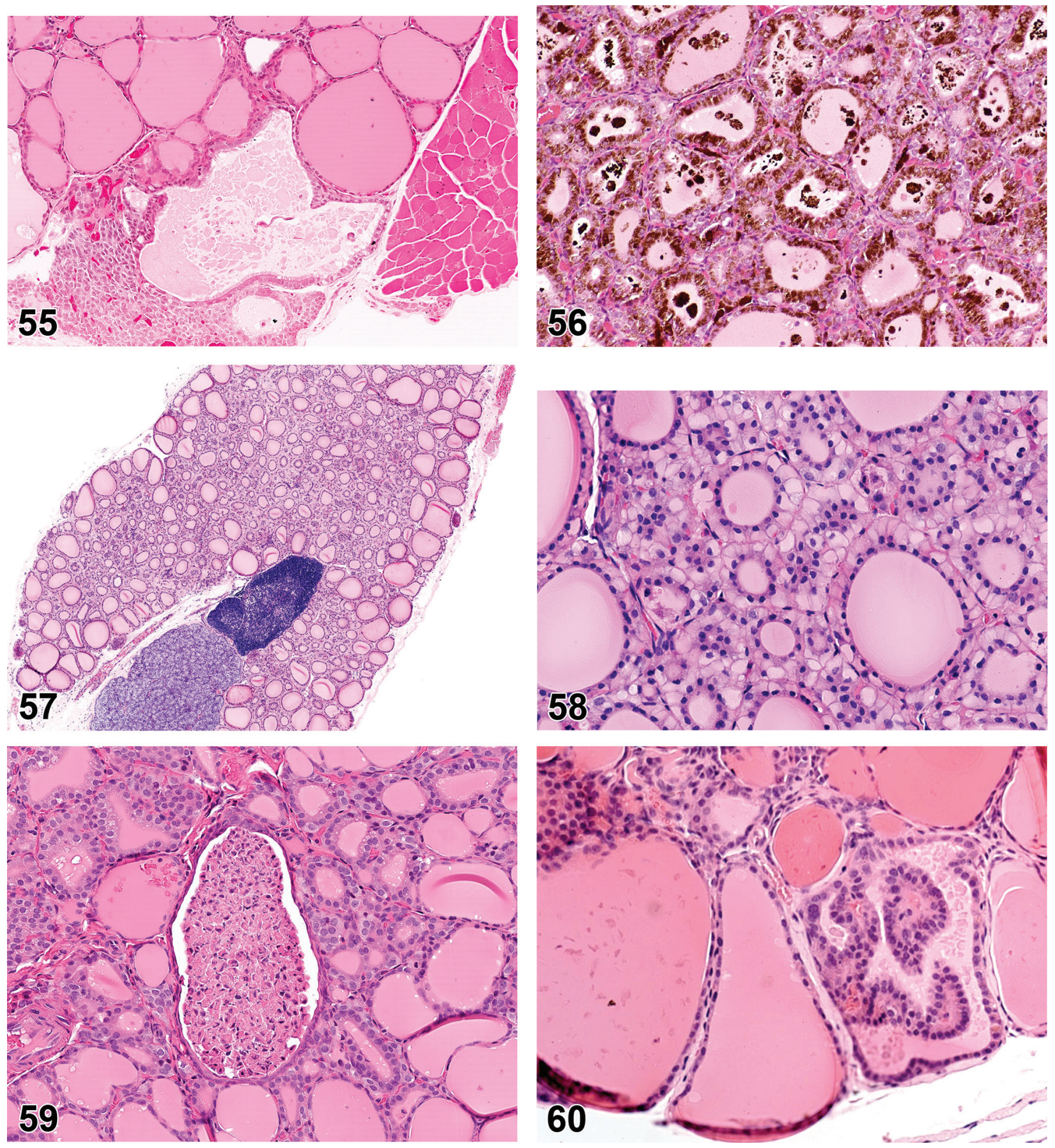

Figure 55.- Rat thyroid gland. Persistent thyroglossal duct.

Figure 56.- Rat thyroid gland. Pigment in the thyroid follicular cells and colloid.

FIGURE 57.- Rat thyroid gland. Thyroid dysplasia and ectopic thymus.

Figure 58.- Rat thyroid gland. Thyroid dysplasia.

FIGURE 59.-Rat thyroid gland. Ultimobranchial cyst.

FIGURE 60.- Rat thyroid gland. Hyperplasia, follicular cell, focal with papillary projections into the colloid. 

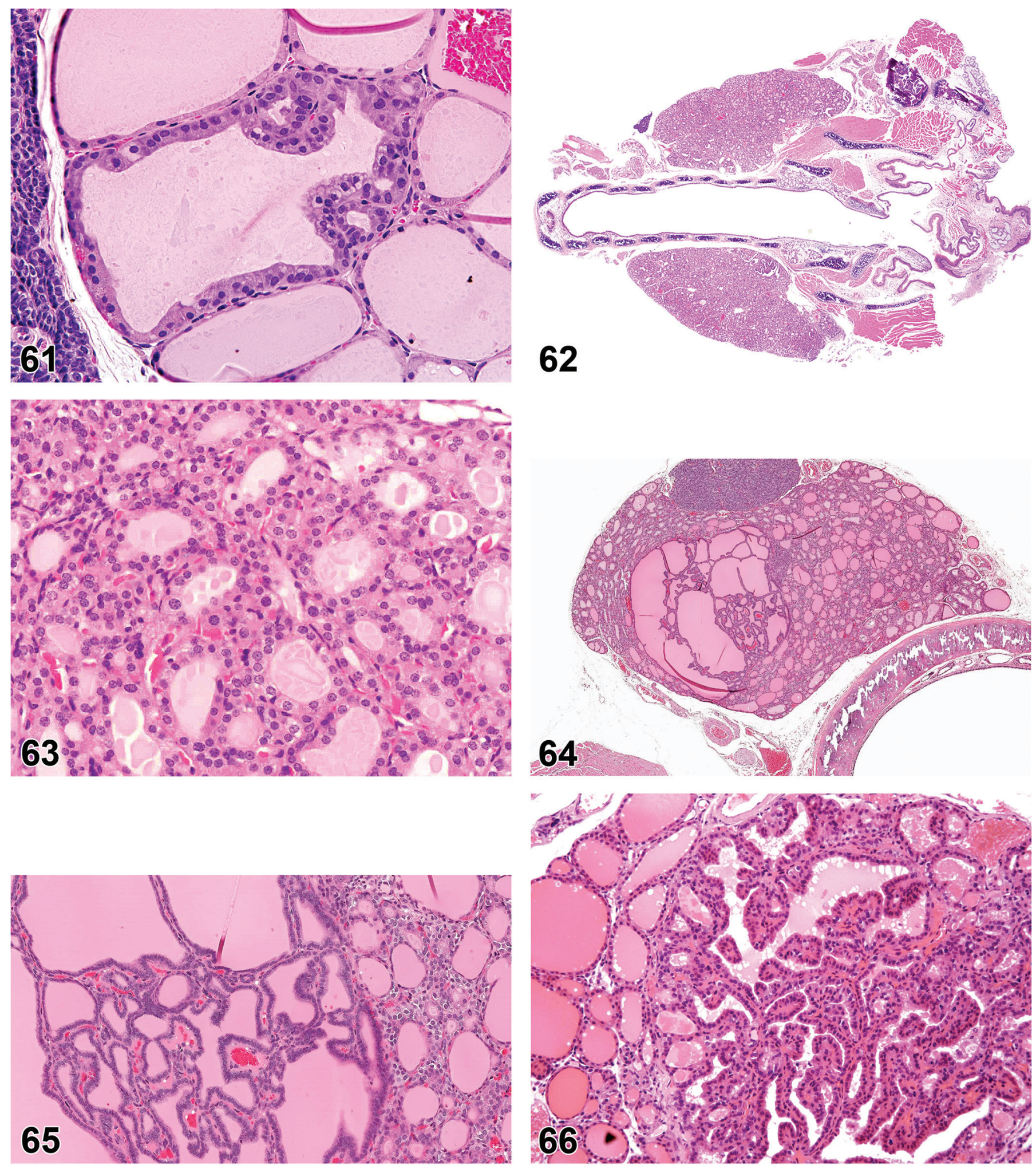

Figure 61.- Mouse thyroid gland. Hyperplasia, follicular cell, focal with piling up of follicular cells.

Figure 62.-Mouse thyroid glands. Hyperplasia, follicular cell, diffuse.

Figure 63.-Mouse thyroid gland. Hyperplasia, follicular cell, diffuse.

Figure 64.- Rat thyroid gland. Adenoma, follicular cell, papillary and cystic.

Figure 65.-Rat thyroid gland. Adenoma, follicular cell, papillary and cystic.

Figure 66.- Mouse thyroid gland. Adenoma, follicular cell, papillary. 

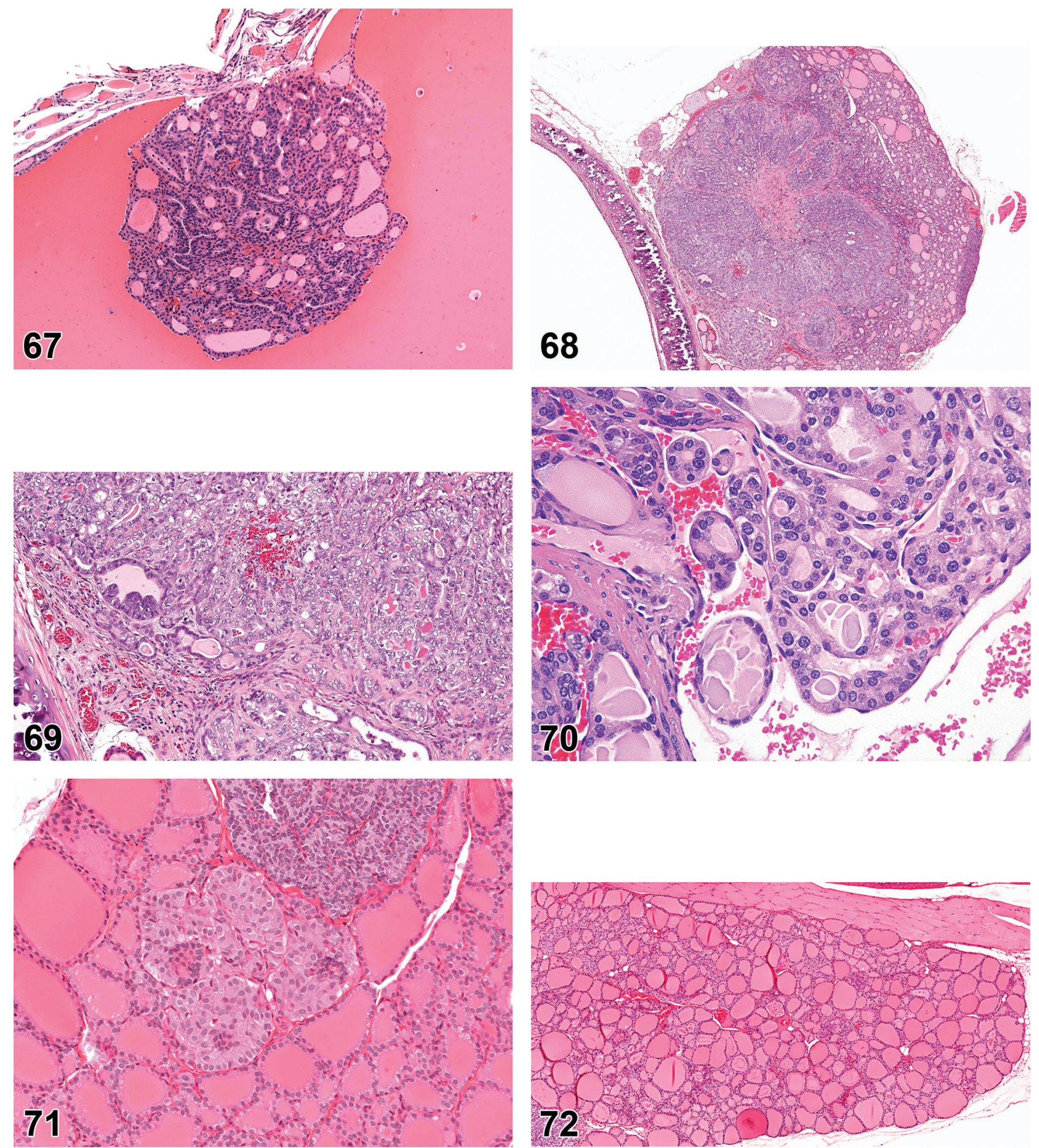

FigURE 67.- Mouse thyroid gland. Adenoma, follicular cell, cystic.

Figure 68.-Rat thyroid gland. Carcinoma, follicular cell.

FIGURE 69.- Rat thyroid gland. Carcinoma, follicular cell with cellular atypia and tissue invasion.

Figure 70.- Rat thyroid gland. Carcinoma, follicular cell with invasion into a blood vessel.

Figure 71. - Rat thyroid gland. C-cell hyperplasia, focal (adjacent to the parathyroid gland that is at the top of the image).

Figure 72.- Rat thyroid gland. C-cell hyperplasia, diffuse. 

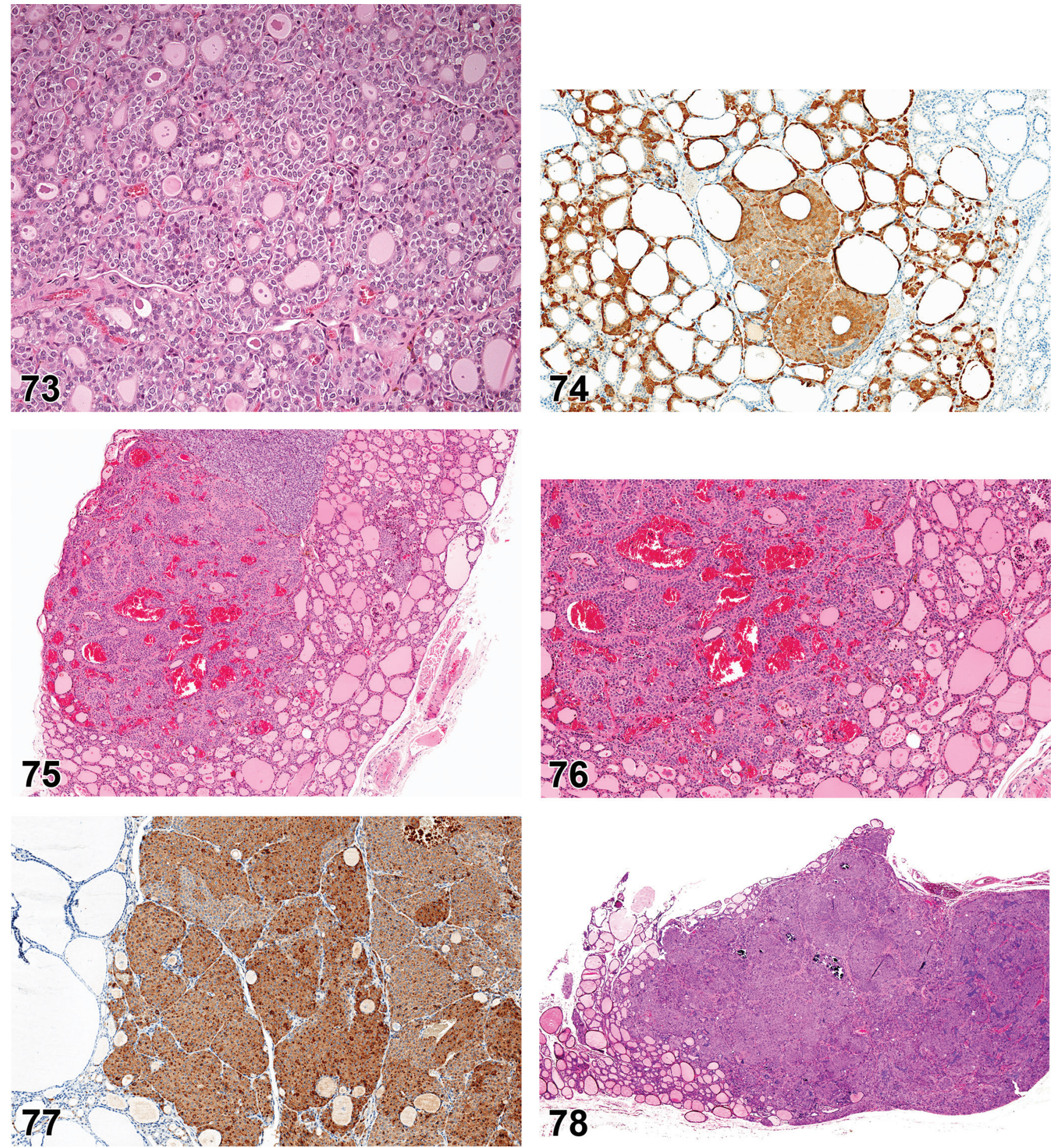

FIGURE 73.-Rat thyroid gland. C-cell hyperplasia, diffuse.

Figure 74.- Rat thyroid gland. C-cell hyperplasia, focal and diffuse, calcitonin immunohistochemistry.

Figure 75.- Rat thyroid gland. C-cell adenoma.

FIGURE 76. - Rat thyroid gland. C-cell adenoma.

FIGURE 77.- Rat thyroid gland. C-cell adenoma, calcitonin immunohistochemistry.

FIGURE 78. - Rat thyroid gland. C-cell carcinoma. 

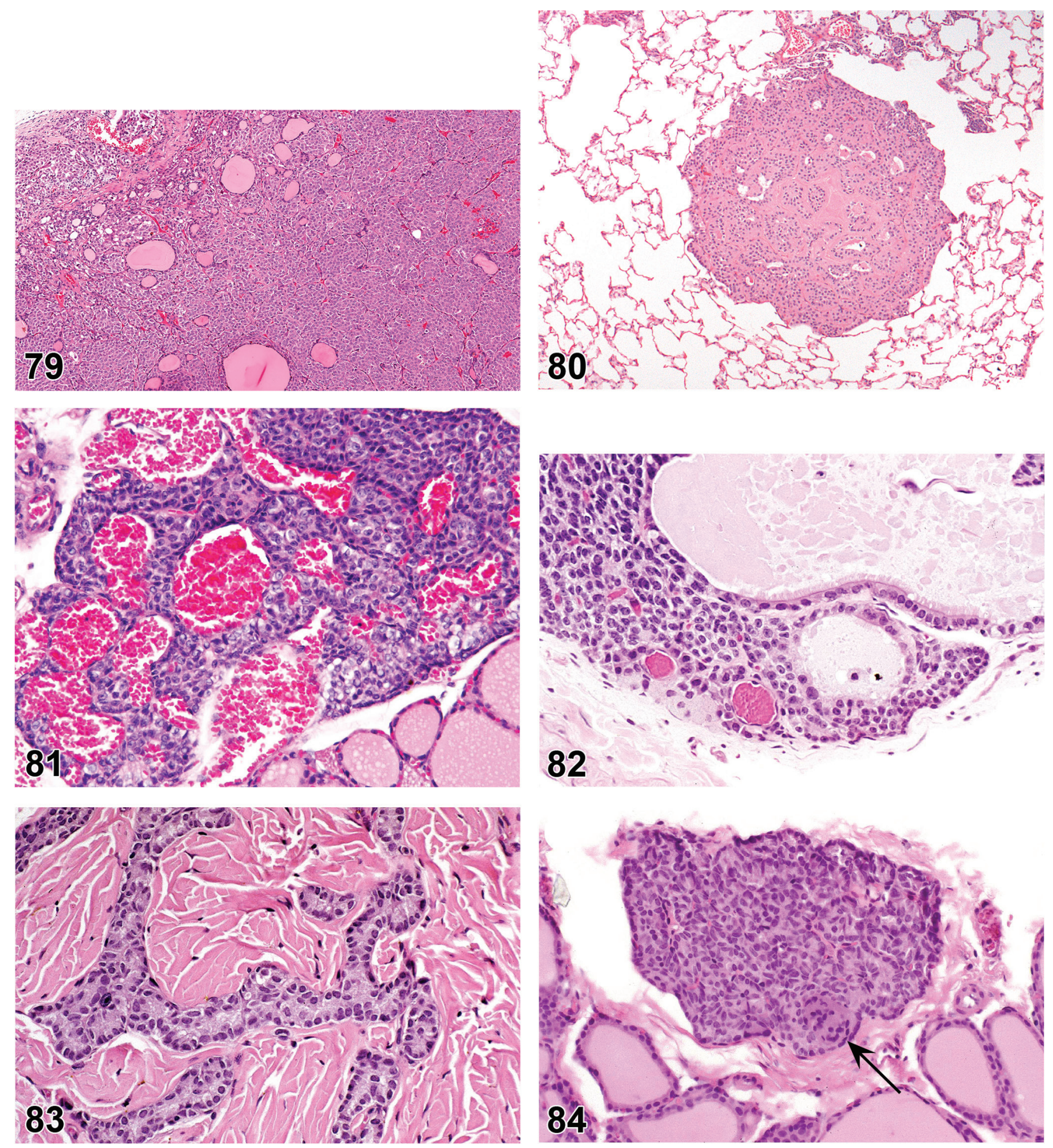

FIGURE 79.- Rat thyroid gland. C-cell carcinoma, capsular invasion.

Figure 80.- Rat lung. C-cell carcinoma, metastasis.

Figure 81.- Mouse parathyroid gland. Angiectasis.

Figure 82.-Mouse parathyroid gland. Cyst.

FIGURE 83.- Rat parathyroid gland. Fibrosis.

FIGURE 84.- Rat parathyroid gland. Multinucleate giant cell (arrow). 

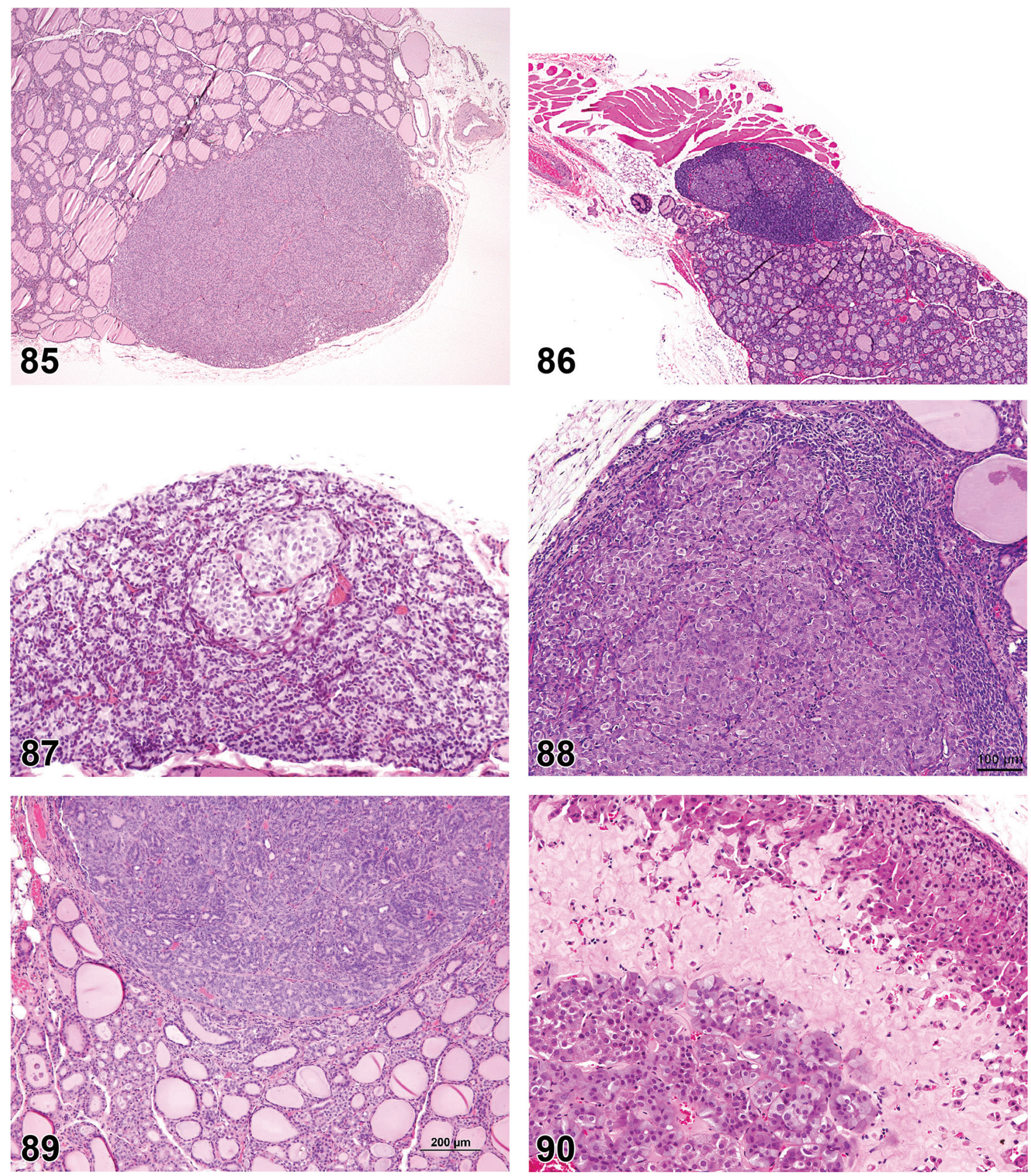

FIGURE 85.-Rat parathyroid gland. Hyperplasia, diffuse.

FIGURE 86.- - Rat parathyroid gland. Hyperplasia, multifocal.

Figure 87.- Rat parathyroid gland. Hyperplasia, focal, water clear cell type.

FIGURE 88.-Rat parathyroid gland. Adenoma.

Figure 89. - Rat parathyroid gland. Carcinoma.

Figure 90.-Mouse adrenal gland. Amyloidosis at the corticomedullary junction. 

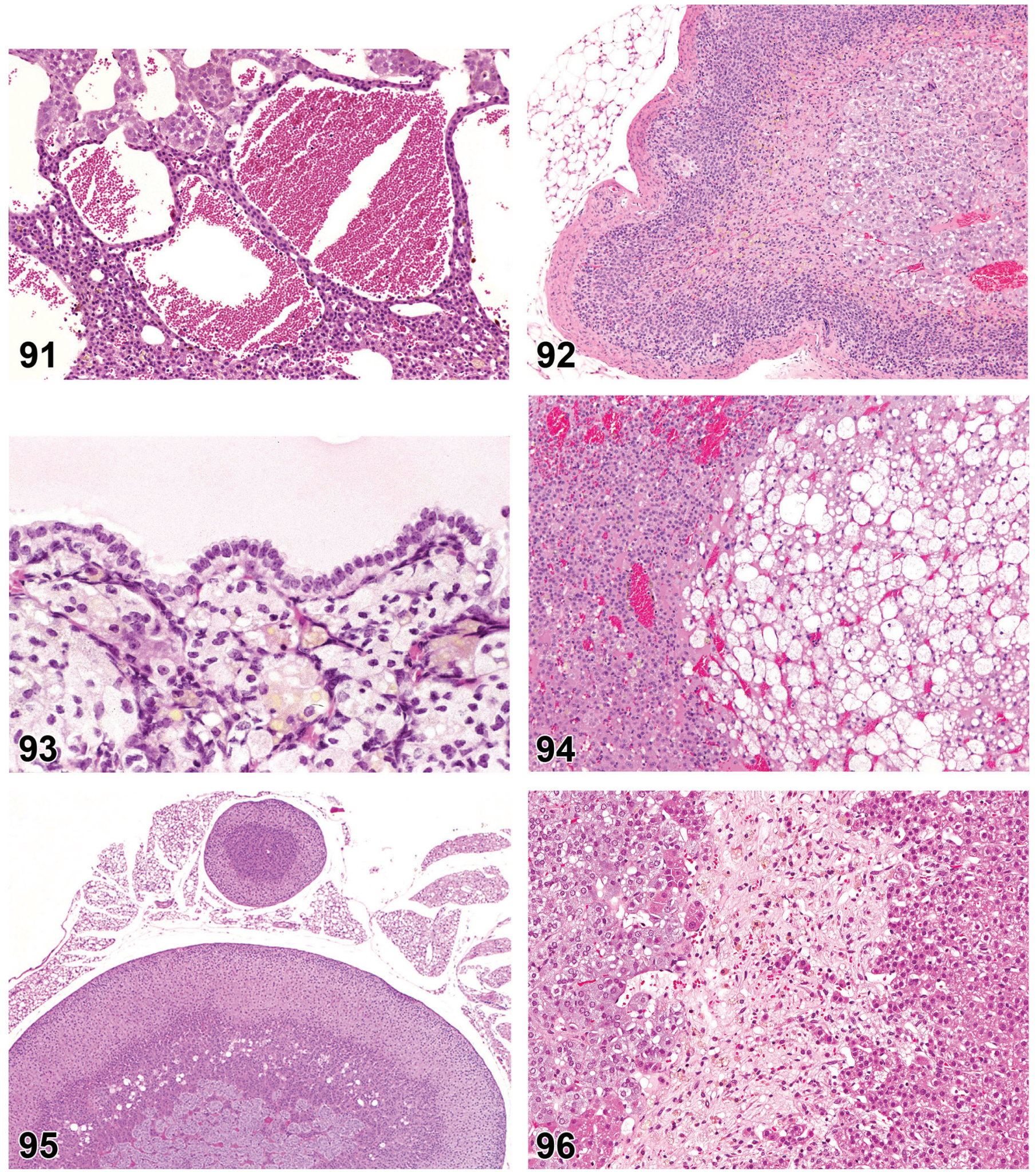

Figure 91.-Rat adrenal cortex. Angiectasis.

Figure 92.- Rat adrenal cortex. Atrophy with pigment in the zona reticularis.

FIGURE 93.- Mouse adrenal cortex. Cyst lined by simple cuboidal to columnar epithelium.

Figure 94.-Rat adrenal cortex. Degeneration, cystic.

Figure 95.- Mouse adrenal gland. Ectopic tissue, containing only cortex at top of the image.

FIGURE 96.-Rat adrenal gland. Fibrosis in the zona reticularis with pigment. 

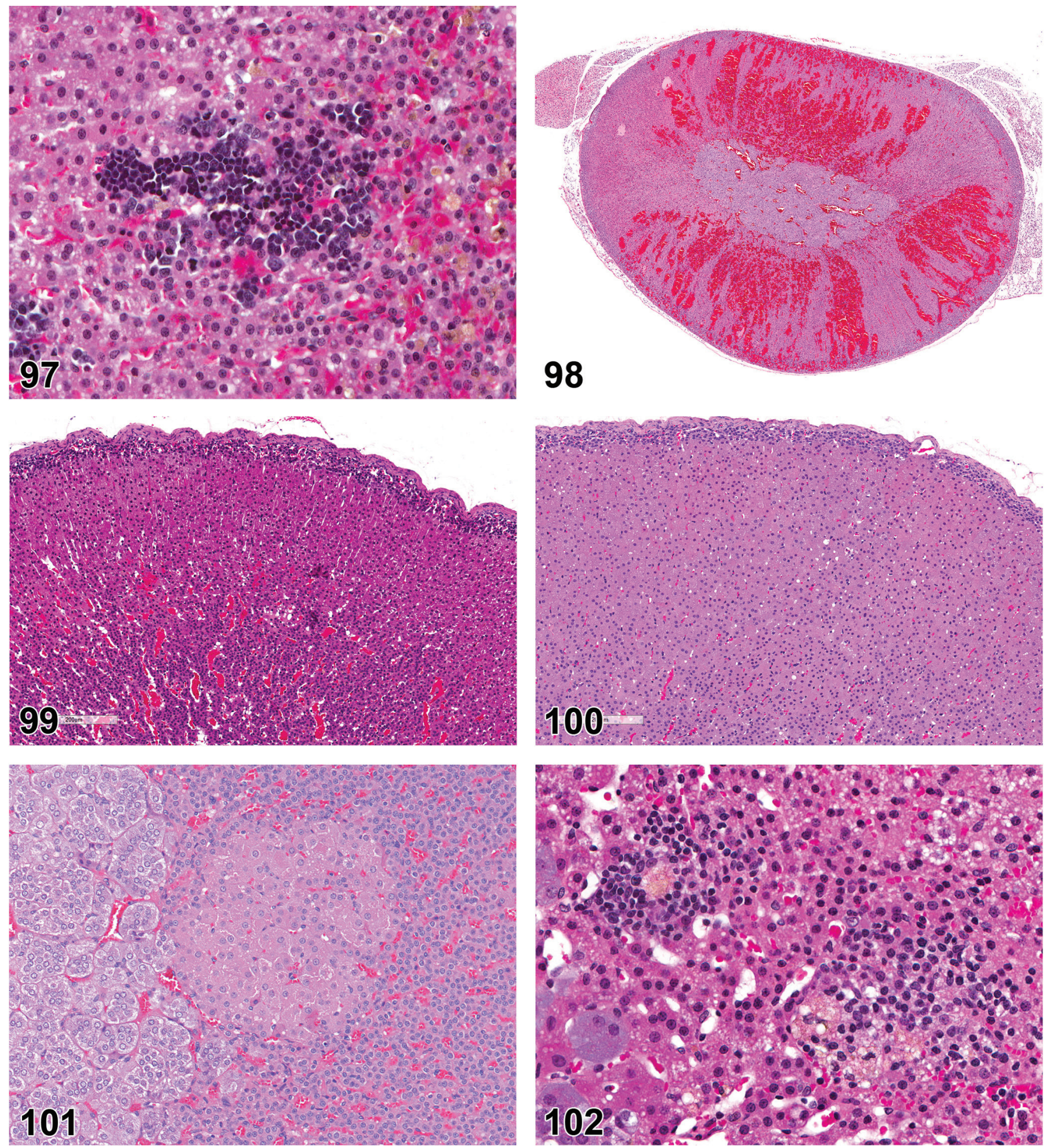

FiguRE 97.- Rat adrenal cortex. Extramedullary hematopoiesis.

Figure 98.- Rat adrenal cortex. Hemorrhage.

FIGURE 99.- - Rat adrenal cortex. Normal control (compare to Figure 100 for hypertrophy, diffuse).

Figure 100.-Rat adrenal cortex. Hypertrophy, cortical, diffuse (compare to Figure 99, control).

FiguRE 101.- Rat adrenal cortex. Hypertrophy, cortical, focal.

Figure 102.-Rat adrenal cortex. Infiltrate, mononuclear. 

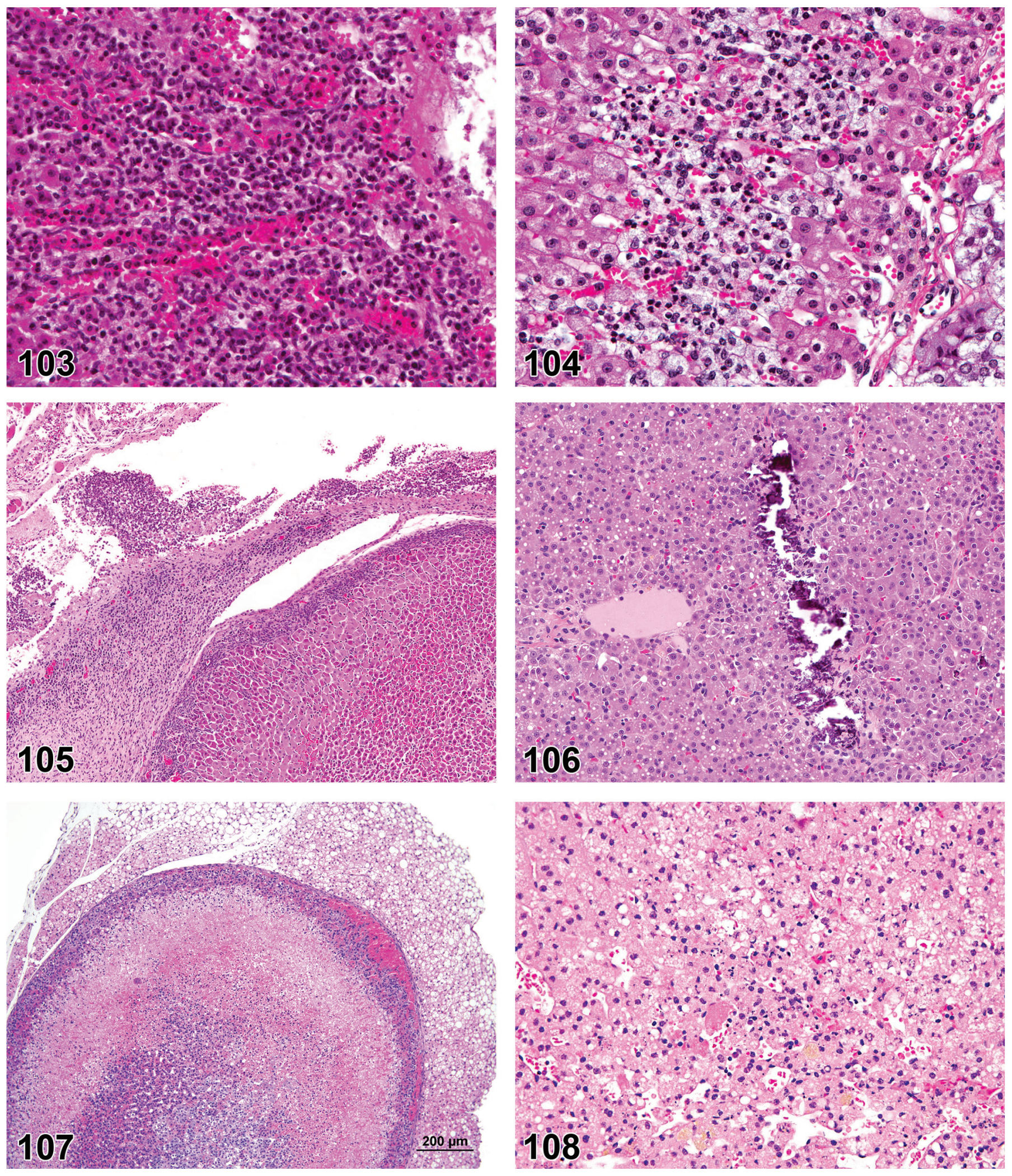

FIGURE 103.-Mouse adrenal cortex. Inflammation, mixed.

FIGURE 104.-Mouse adrenal cortex. Inflammation, suppurative.

FIGURE 105.-Mouse adrenal cortex. Inflammation, suppurative with secondary peritonitis.

FIGURE 106.--Rat adrenal cortex. Mineralization.

FIGURE 107.-Mouse adrenal cortex. Necrosis, zona fasiculata and reticularis.

Figure 108.-Rat adrenal cortex. Necrosis, zona fasiculata. 


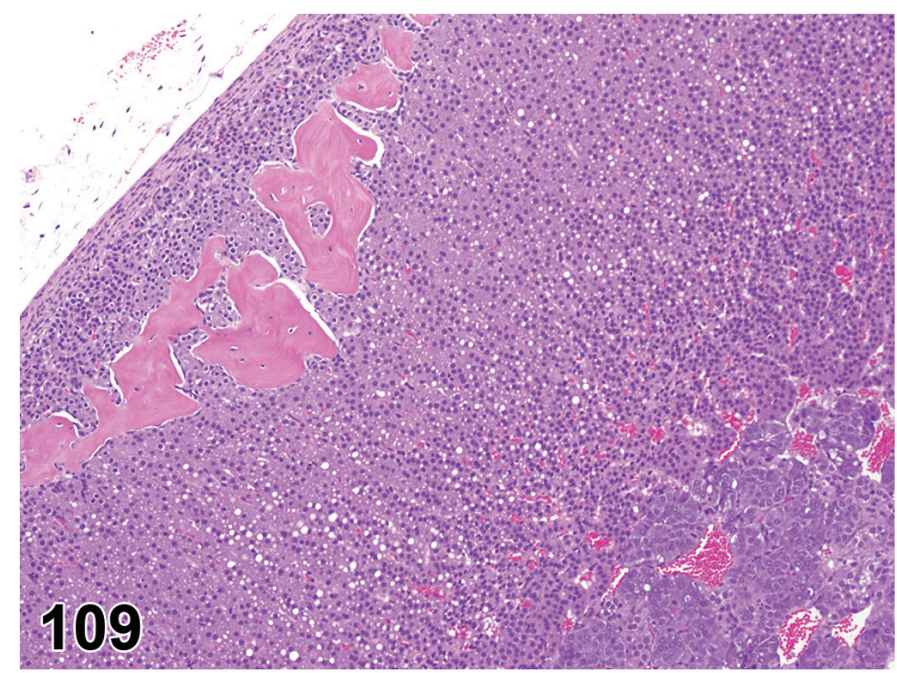

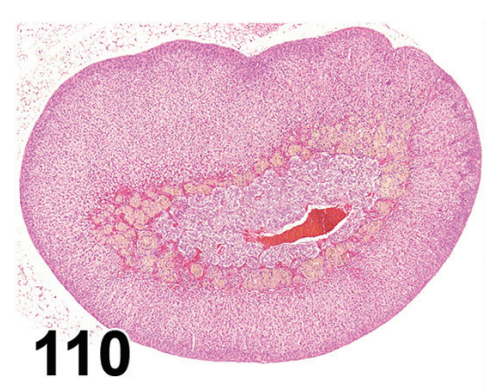
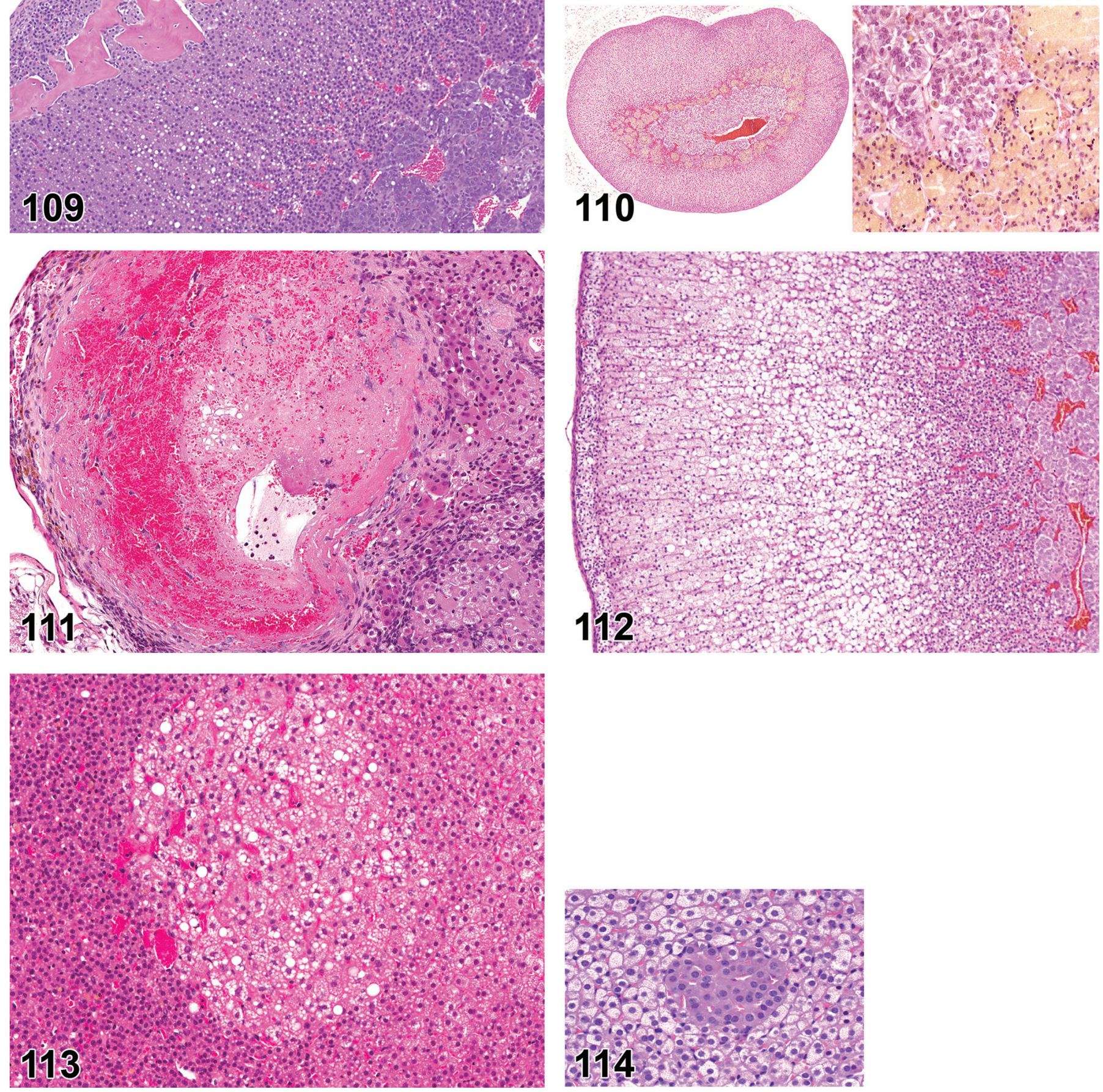

Figure 109.- Rat adrenal cortex. Osseous metaplasia.

Figure 110.-Mouse adrenal gland. Pigmentation, X-zone.

Figure 111.-Mouse adrenal cortex. Thrombosis.

Figure 112.-Rat adrenal cortex. Vacuolation, cortical, increased, diffuse. Figure 113.- Rat adrenal cortex. Vacuolation, cortical, increased, focal. FIGURE 114.-Rat adrenal cortex. Vacuolation, cortical, decreased, focal. 

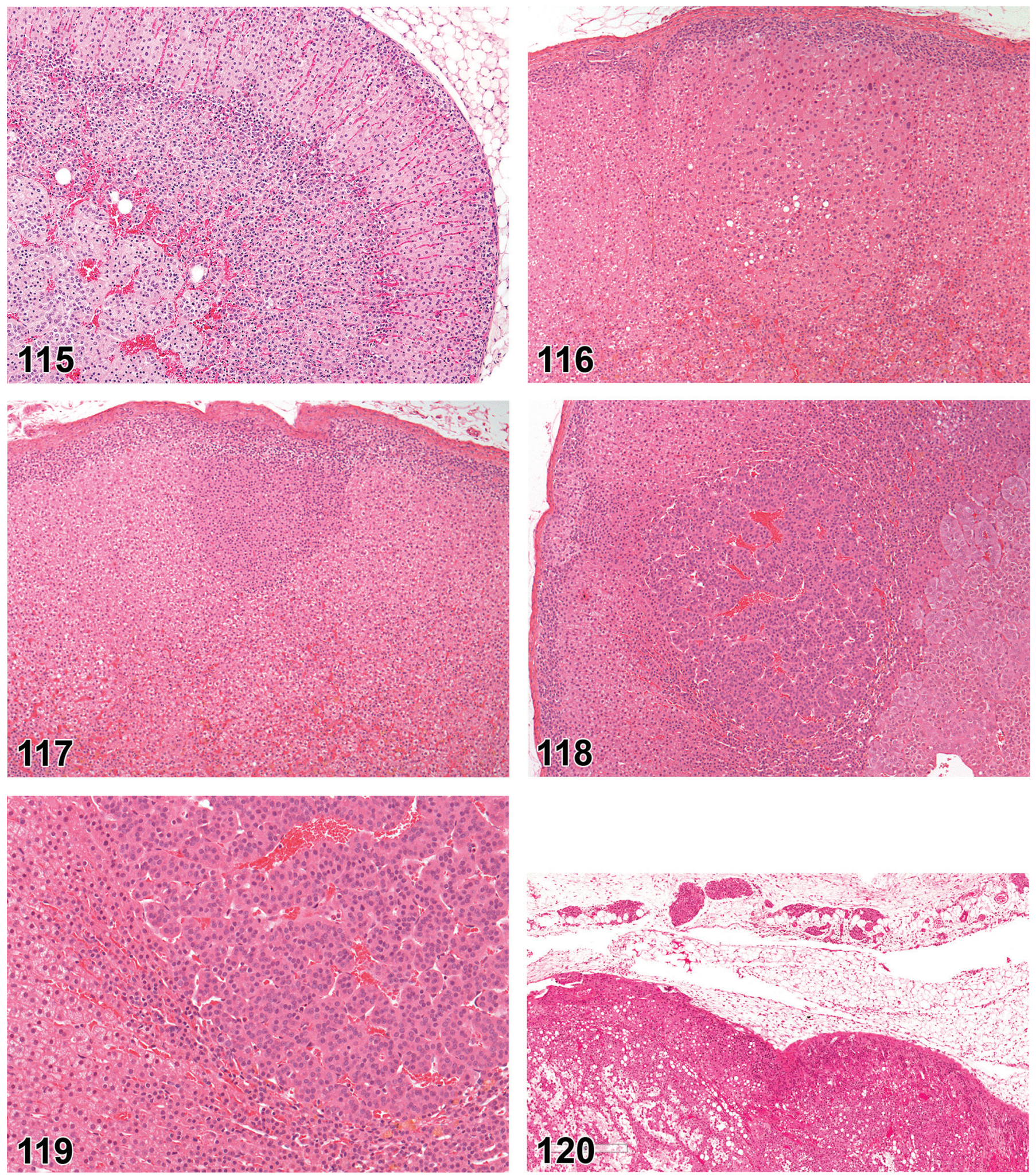

Figure 115.-Mouse adrenal cortex. X-zone persistence.

Figure 116.-Rat adrenal cortex. Hyperplasia, cortical, focal, large cells.

FIGURE 117.- Rat adrenal cortex. Hyperplasia, cortical, focal, small cells.

Figure 118.-Rat adrenal cortex. Adenoma, cortical.

Figure 119.- Rat adrenal cortex. Adenoma, cortical.

FIGURE 120.-Rat adrenal cortex. Carcinoma, cortical with invasion into surrounding adipose tissue. 

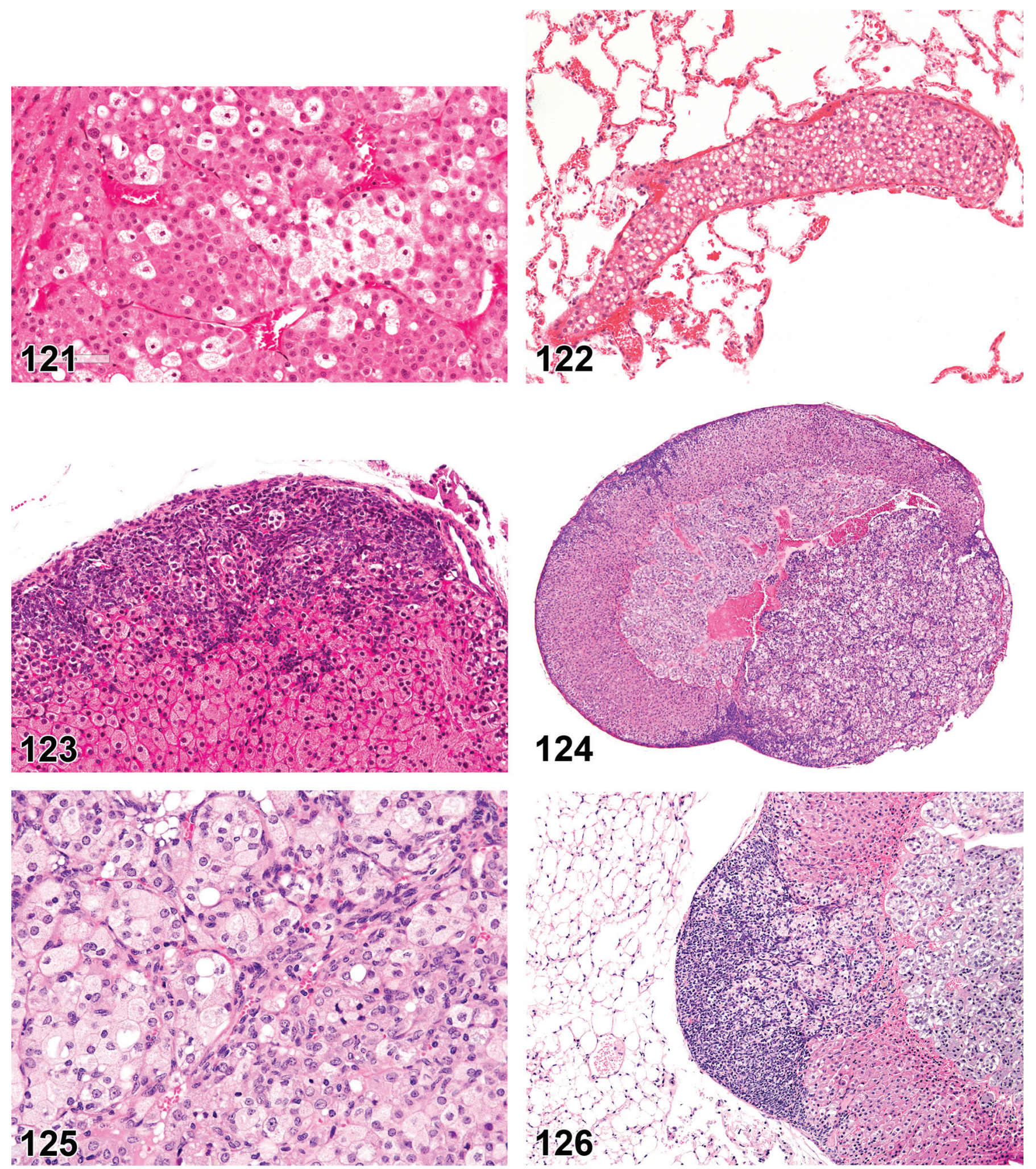

Figure 121.-Rat adrenal cortex. Carcinoma, cortical.

Figure 122.-Rat lung. Adrenal carcinoma, cortical, intravascular metastasis.

FigURE 123.--Mouse adrenal cortex. Hyperplasia, subcapsular cell.

FiguRE 124.-Mouse adrenal cortex. Adenoma, subcapsular cell, type B.

Figure 125.-Mouse adrenal cortex. Adenoma, subcapsular cell, type B.

FIGURE 126.-Mouse adrenal cortex. Adenoma, subcapsular cell, type A. 

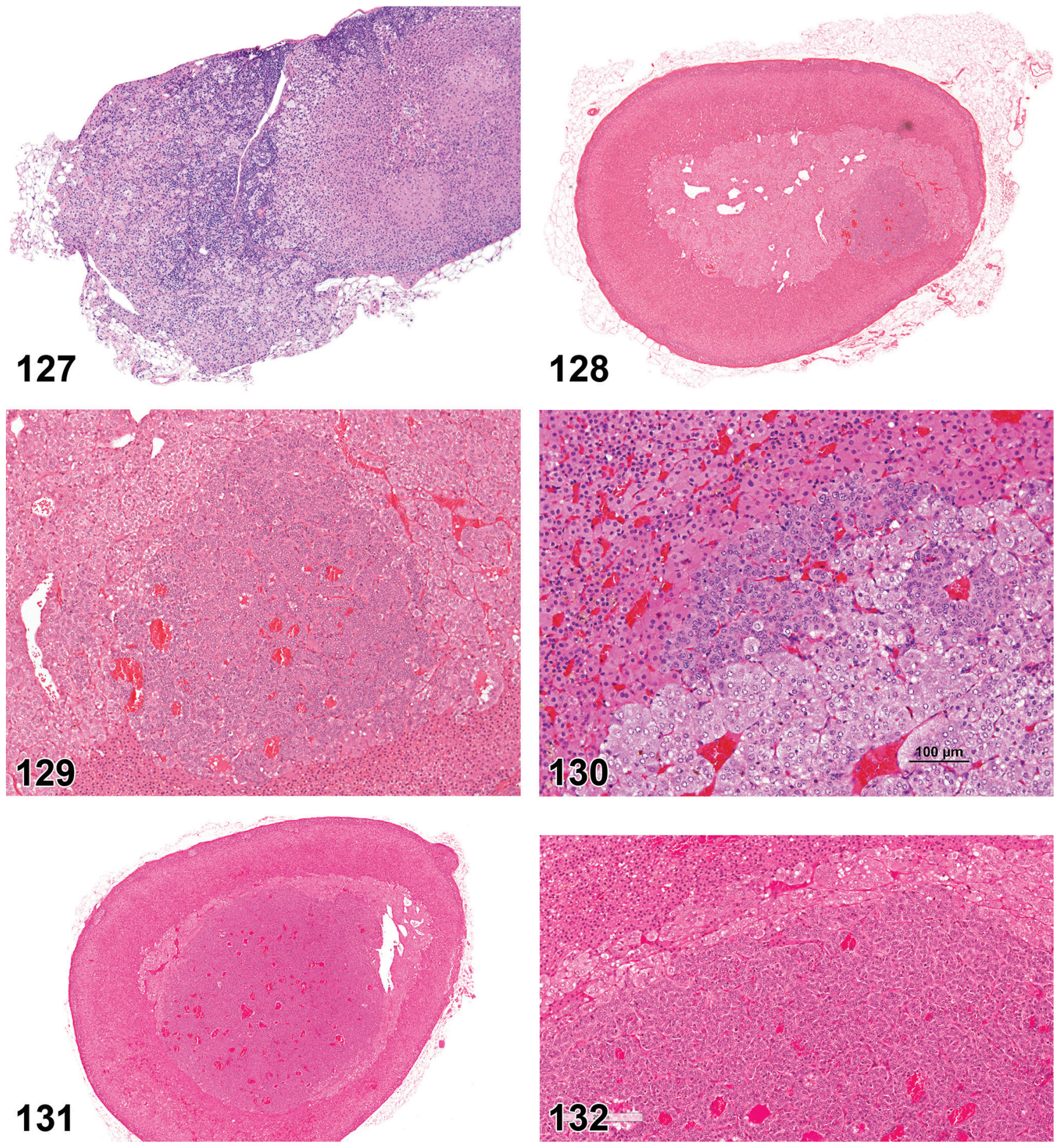

Figure 127.- Mouse adrenal cortex. Carcinoma, subcapsular cell, mixed type A \& B.

FIgURE 128.-Rat adrenal medulla. Hyperplasia, medullary, focal.

FIGURE 129.-Rat adrenal medulla. Hyperplasia, medullary, focal.

Figure 130.-Rat adrenal medulla. Hyperplasia, medullary, focal.

Figure 131.-Rat adrenal medulla. Pheochromocytoma, benign.

FIGURE 132.-Rat adrenal medulla. Pheochromocytoma, benign. 

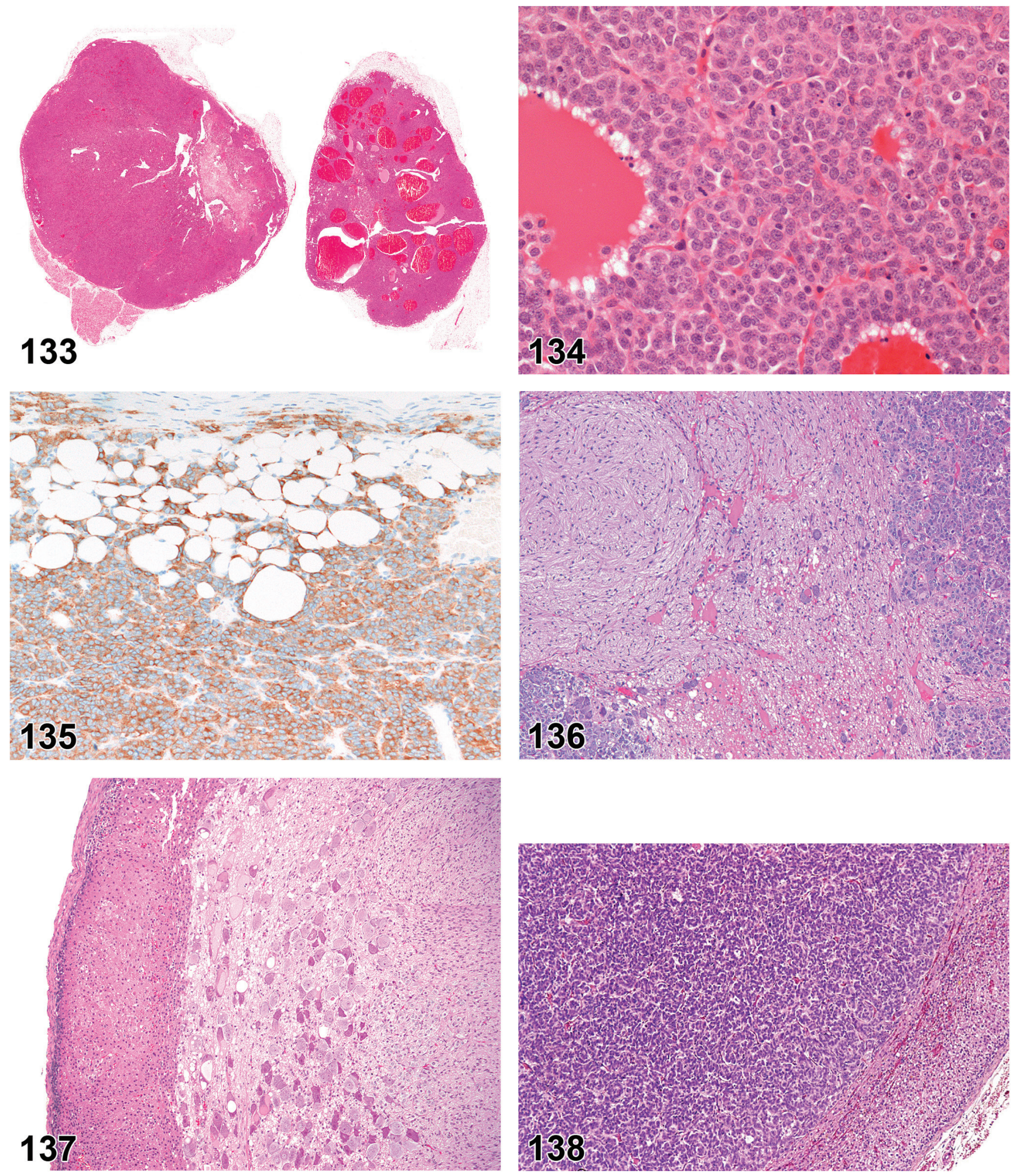

Figure 133.-Rat adrenal medulla. Pheochromocytoma, malignant with extracapsular invasion.

Figure 134.-Rat adrenal medulla. Pheochromocytoma, malignant.

FIGURE 135.-Rat adrenal medulla. Pheochromocytoma, malignant with immunohistochemistry for synaptophysin.

Figure 136.-Rat adrenal medulla. Pheochromocytoma, complex, benign.

FigURE 137.-Rat adrenal medulla. Ganglioneuroma, benign.

Figure 138.-Rat adrenal medulla. Neuroblastoma. 

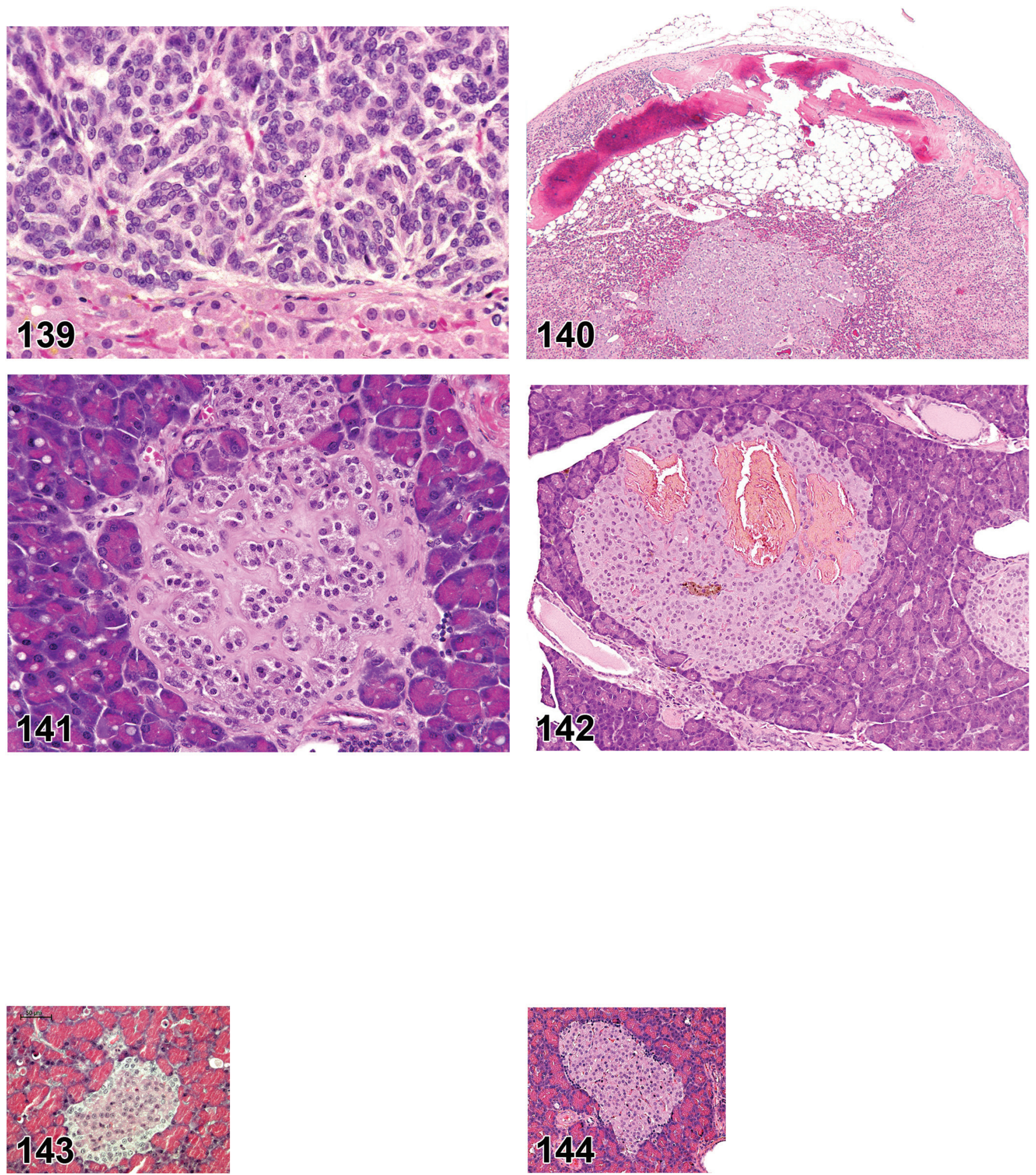

FIGURE 139.-Rat adrenal medulla. Neuroblastoma with pseudorosettes.

Figure 140.-Rat adrenal cortex. Myelolipoma.

Figure 141.-Mouse endocrine pancreas. Amyloid, islet.

Figure 142.-Rat endocrine pancreas. Angiectasis, islet.

Figure 143. - Rat endocrine pancreas. Atrophy, islet, $\beta$ cells. The atrophic $\beta$ cells in the central region of the islet have eosinophilic cytoplasm and the islets are irregular in shape and shrunken. Apoptosis is present in the surrounding exocrine pancreas.

FIgURE 144.-Rat endocrine pancreas. Atrophy, islet, $\alpha$ cells. The atrophic $\alpha$ cells at the islet periphery have a reduced amount of cytoplasm and nuclear condensation. 

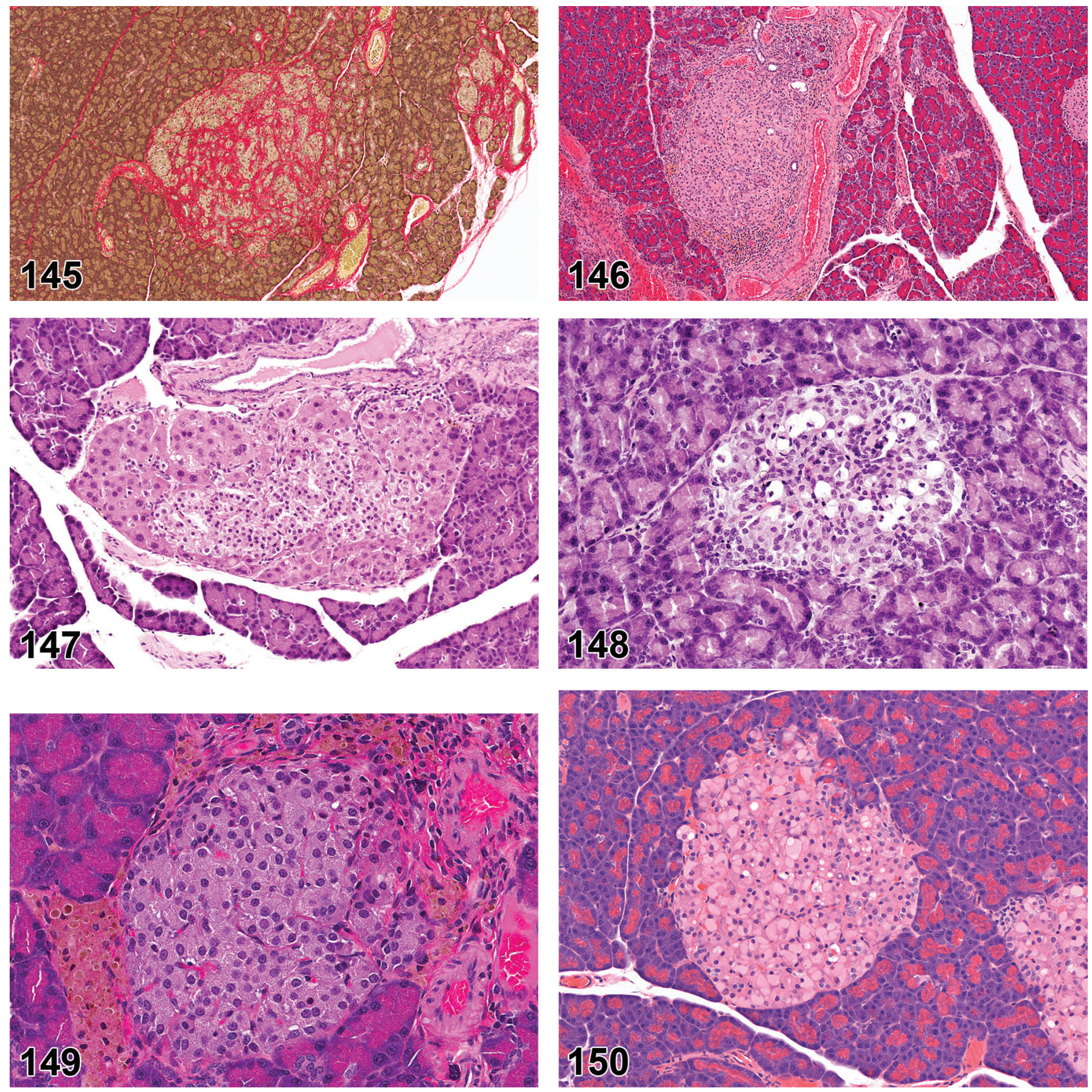

FIGURE 145.-Rat endocrine pancreas. Fibrosis, islet, Sirius red stain.

FIGURE 146.-Rat endocrine pancreas. Inflammation, islet, mononuclear with fibrosis and pigment.

FiguRE 147.-Rat endocrine pancreas. Metaplasia, hepatocyte.

Figure 148.-Rat endocrine pancreas. Necrosis, islet.

Figure 149.- Rat endocrine pancreas. Pigment, islet.

FIGURE 150.-Rat endocrine pancreas. Vacuolation, islet. 

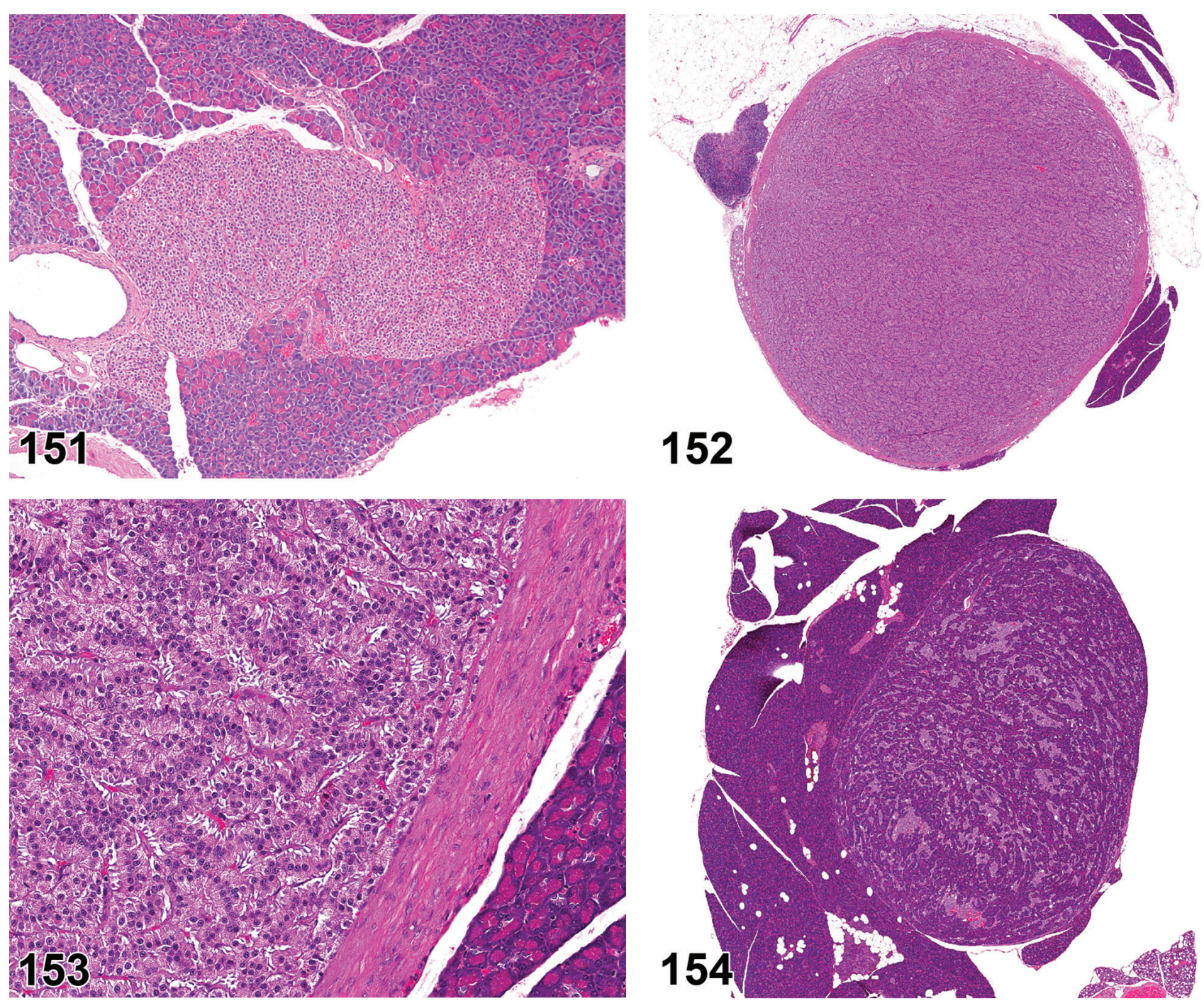

Figure 151.-Rat endocrine pancreas. Hyperplasia, islet.

Figure 152.-Rat endocrine pancreas. Adenoma, islet.

FIGURE 153.-Rat endocrine pancreas. Adenoma, islet with a fibrous capsule.

FIgURE 154.-Rat endocrine pancreas. Adenoma, islet-acinar. 

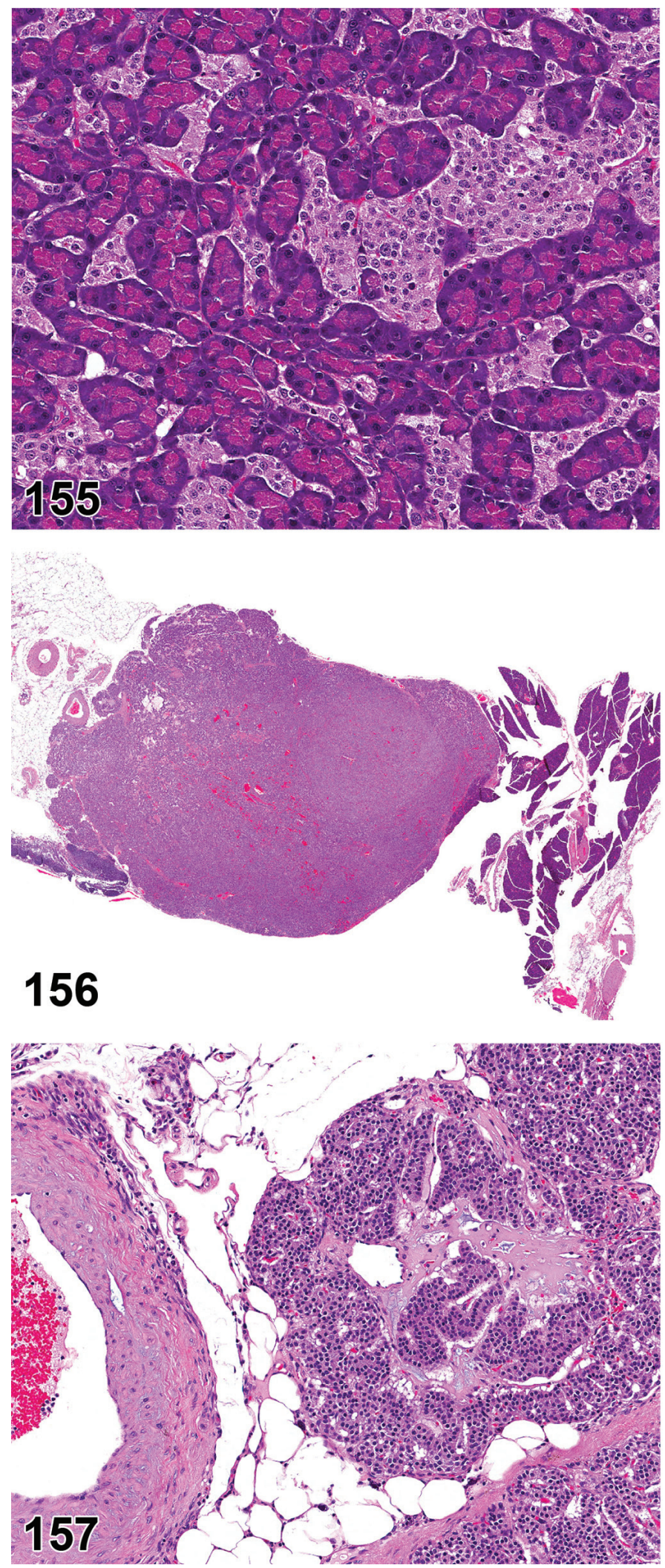

FiguRE 155.-Rat endocrine pancreas. Adenoma, islet-acinar.

FIGURE 156.-Rat endocrine pancreas. Carcinoma, islet.

FIGURE 157.-Rat endocrine pancreas. Carcinoma, islet. 


\section{REFERENCES}

Abe M, Hayashi S, Usuda K, Hagio S, Furukawa S, and Nakae D. Carcinogen-induced thyroid proliferative lesions in Wistar Hannover GALAS rats with thyroid dysplasia. J Toxicol Pathol. 25: 11-17. 2012. [Medline] [CrossRef]

Ackermann MR. Cellular and tissue responses to injury. In: Pathologic Basis of Veterinary Disease. Zachary, JF and McGavin, MD (eds). $5^{\text {th }}$ ed. Elsevier, St. Louis, MO. pp. 51-57, 2012.

Adeghate E, Hameed RS, Ponery AS, Tariq S, Sheen RS, Shaffiullah M, and Donáth T. Streptozotocin causes pancreatic beta cell failure via early and sustained biochemical and cellular alterations. Exp Clin Endocrinol Diabetes. 118: 699-707. 2010. [Medline] [CrossRef]

Aguzzi A, Wagner EF, Williams RL, and Courtneidge SA. Sympathetic hyperplasia and neuroblastomas in transgenic mice expressing polyoma middle T antigen. New Biol. 2: 533-543. 1990. [Medline]

Aigelsreiter A, Janig E, Stumptner C, Fuchsbichler A, Zatloukal K, and Denk H. How a cell deals with abnormal proteins. Pathogenetic mechanisms in protein aggregation diseases. Pathobiology. 74: 145-158. 2007. [Medline] [CrossRef]

Al Zubaidy AJ, and Malinowski W. Spontaneous pineal body tumours (pinealomas) in Wistar rats; a histological and ultrastructural study. Lab Anim. 18: 224-229. 1984. [Medline] [CrossRef]

Allen DJ, DiDio LJA, Gentry ER, and Ohtani O. The aged rat pineal gland as revealed in SEM and TEM. Age (Omaha). 5: 119-126. 1982. [CrossRef]

Alonso G, Runquist M, Hussy N, Duvoid A, and Moos F. Age-related modifications of the morphological organization of pituicytes are associated with alteration of the GABAergic and dopaminergic innervation afferent to the neurohypophysial lobe. Eur J Neurosci. 18: 1889-1903. 2003. [Medline] [CrossRef]

Altenähr E, and Jenke W. Experimental parathyroiditis in the rat by passive immunisation. Virchows Arch A Pathol Anat Histol. 363: 333-342. 1974. [Medline] [CrossRef]

Anderson MP, and Capen CC. The endocrine system, pituitary. In: Pathology of laboratory animals. Anderson, MP, Benirschke, K, Garner, FM and Jones, TC (eds). Springer-Verlag, New York. pp. 424-433, 1978.

Apps JR, and Martinez-Barbera JP. Genetically engineered mouse models of craniopharyngioma: an opportunity for therapy development and understanding of tumor biology. Brain Pathol. 27: 364-369. 2017. [Medline] [CrossRef]

Armocida AD, Itagaki S-I, Kume E, and Doi K. Hepatic lesion in streptozotocin (SZ)-induced diabetic mice. J Toxicol Pathol. 7: 87-90. 1994. [CrossRef]

Asamoto M, Hokaiwado N, Cho YM, Ikeda Y, Takahashi S, and Shirai T. Metastasizing neuroblastomas from taste buds in rats transgenic for the Simian virus 40 large $\mathrm{T}$ antigen under control of the probasin gene promoter. Toxicol Pathol. 29: 363-368. 2001. [Medline] [CrossRef]

Attia MA. Neoplastic and nonneoplastic lesions in aging female rats with special reference to the functional morphology of the hyperplastic and neoplastic changes in the pituitary gland. Arch Toxicol. 57: 77-83. 1985. [Medline] [CrossRef]

Bartels T, Wäse K, Heinrichs M, Stolte M, Roome N, Scherer P, and Lindauer $\mathrm{K}$. Regulatory forum opinion piece: review-toxicological pathology profile and regulatory expectations for nonclinical development of insulins and insulin analogues. Toxicol Pathol. 44: 931-946. 2016. [Medline] [CrossRef]

Belloni AS, Musajo FG, Boscaro M, DAgostino D, Rebuffat P, Cavallini L, Mazzocchi G, and Nussdorfer GG. An ultrastructural stereological study of accessory adrenocortical glands in bilaterally adrenalectomised rats. $J$ Anat. 165: 107-120. 1989. [Medline]

Bertolino P, Tong WM, Herrera PL, Casse H, Zhang CX, and Wang ZQ. Pancreatic beta-cell-specific ablation of the multiple endocrine neoplasia type 1 (MEN1) gene causes full penetrance of insulinoma development in mice. Cancer Res. 63: 4836-4841. 2003. [Medline]

Bomhard E. Frequency of spontaneous tumours in NMRI mice in 21-month studies. Exp Toxicol Pathol. 45: 269-289. 1993. [Medline] [CrossRef]
Boorman GA, De Lellis RA, and Elwell MR. C-Cell Hyperplasia, C-Cell Adenoma, and C-Cell Carcinoma. In: Monographs on pathology of laboratory animals. Endocrine system. Jones, TC, Capen, CC and Mohr, U (eds). $2^{\text {nd }}$ ed. Springer, Berlin; New York. pp. 262-274, 1996.

Boorman GA, and Elwell MR. Follicular Cell Hyperplasia, Adenoma, and Carcinoma, Thyroid Gland. In: Monographs on pathology of laboratory animals. Endocrine system. Jones, TC, Capen, CC and Mohr, U (eds). $2^{\text {nd }}$ ed. Springer, Berlin; New York. pp. 244-254, 1996.

Boorman GA, and Eustis SL. Neoplasms of the exocrine pancreas. In: Atlas of tumor pathology of the Fischer rat. Stinson, SF, Schuller, HM and Reznik, G (eds). CRC Press, Boca Raton, FL. pp. 222-225, 1990.

Boorman GA, and Sills RC. Exocrine and Endocrine Pancreas. In: Pathology of the Mouse. Maronpot, RR, Boorman, G.A., Gaul, B.W. (ed) Cache River Press, Vienna, Illinois, USA. pp. 185-205, 1999.

Bossé R, Fumagalli F, Jaber M, Giros B, Gainetdinov RR, Wetsel WC, Missale $\mathrm{C}$, and Caron MG. Anterior pituitary hypoplasia and dwarfism in mice lacking the dopamine transporter. Neuron. 19: 127-138. 1997. [Medline] [CrossRef]

Botts S, Capen CC, DeLellis RA, Deschl U, Hartig F, Karbe E, Konishi Y, Krinke GJ, Landes C, Mettler F, Rebel W, Riley MGI, Tuch K, and Urwyler H. International classification of rodent tumours. Part I: The Rat. In: IARC scientific publications, Mohr, U (ed) International Agency for Research on Cancer; Oxford University Press, Lyon; New York. pp. 1-75, 1994.

Botts S, Jokinen MP, Isaacs KR, Meuten DJ, and Tanaka N. Proliferative Lesions of the Thyroid and Parathyroid Glands. In: Guides for Toxicologic Pathology. STP/ARP/AFIP, Washington, DC. pp. 1-12, 1991.

Brandão RA, Braga MH, de Souza AA, Reis BL, and Faraj de Lima FB. Pituicytoma. Surg Neurol Int. 1: 79. 2010. [Medline] [CrossRef]

Burek JD. Age-associated pathology. In: Pathology of the Aging Rat. CRC Press, West Palm, Beach, FL. pp. 38-41, 1978.

Calvo J, and Boya J. Postnatal evolution of the rat pineal gland: light microscopy. J Anat. 138: 45-53. 1984. [Medline]

Capella C, Oberg K, Papotti M, Volante M, and Bordi C. Mixed exocrineendocrine carcinomas. In: Pathology and genetics of tumours of endocrine organs. World Health Organization classification of tumours. Delellis, RA, Lloyd, RV, Heitz, PU and Eng, C (eds). IARC Press, Lyon. pp. 205-206, 2004.

Capen CC. Functional and fine structural relationships of parathyroid glands. Adv Vet Sci Comp Med. 19: 249-286. 1975. [Medline]

Capen CC. Neoplasms of parathyroid glands. In: Atlas of tumor pathology of the Fischer rat. Stinson, SF, Schuller, HM and Reznik, G (eds). CRC Press, Boca Raton, Florida (USA). pp. 368-378, 1990.

Capen CC. Functional and Pathologic Interrelationships of the Pituitary Gland and Hypothalamus in Animals. In: Monographs on pathology of laboratory animals. Endocrine system. Jones, TC, Capen, CC and Mohr, U (eds). $2^{\text {nd }}$ ed. Springer, Berlin; New York. pp. 3-33, 1996a.

Capen CC. Hormonal Imbalances and Mechanism of Chemical Injury of Thyroid Gland. In: Monographs on pathology of laboratory animals. Endocrine system. Jones, TC, Capen, CC and Mohr, U (eds). $2^{\text {nd }}$ ed. Springer, Berlin; New York. pp. 217-238, 1996b.

Capen CC. Pathobiology of Parathyroid Gland Structure and Function in Animals. In: Monographs on pathology of laboratory animals. Endocrine system. Jones, TC, Capen, CC and Mohr, U (eds). $2^{\text {nd }}$ ed. Springer, Berlin; New York. pp. 293-325, 1996c.

Capen CC. Mechanistic data and risk assessment of selected toxic end points of the thyroid gland. Toxicol Pathol. 25: 39-48. 1997. [Medline] [CrossRef]

Capen CC, Karbe E, and Deschl U. C., G, Germann, P-G, Gopinath, C, Hardisty, JF, Kanno, J, Kaufmann, W, Krinke, G, Küttler, K, Kulwich, B, Landes, C, Lenz, B, Longeart, L, Paulson, E, Sanders, E, and Tuch, K. Endocrine System. In: International classification of rodent tumors. Part II. The mouse. Mohr, U (ed) Springer: WHO-International Agency for Research on Cancer, Berlin; New York. pp. 269-322, 2001.

Capen CC, and Martin SL. The effects of xenobiotics on the structure and function of thyroid follicular and C-cells. Toxicol Pathol. 17: 266-293. 1989. [Medline] [CrossRef] 
Capen CC, and Rosol TJ. Recent advances in the structure and function of the parathyroid gland in animals and the effects of xenobiotics. Toxicol Pathol. 17: 333-345. 1989. [Medline] [CrossRef]

Capen CC, and Rosol TJ. Pathobiology of parathyroid hormone and parathyroid hormone-related protein: introduction and evolving concepts. Monogr Pathol. 35: 1-33. 1993. [Medline]

Carlton WW, and Gries CL. Inflammation, Pituitary Gland: Rat, Mouse, and Hamster. In: Monographs on pathology of laboratory animals. Endocrine system. Jones, TC, Capen, CC and Mohr, U (eds). $2^{\text {nd }}$ ed. Springer, Berlin; New York. pp. 109-110, 1996.

Chamanza R, Marxfeld HA, Blanco AI, Naylor SW, and Bradley AE. Incidences and range of spontaneous findings in control cynomolgus monkeys (Macaca fascicularis) used in toxicity studies. Toxicol Pathol. 38: 642-657. 2010. [Medline] [CrossRef]

Chandra S, Hoenerhoff MJ, and Peterson R. Endocrine System. In: Toxicologic Pathology: Nonclinical Safety Assessment. Sahota, PS, Popp, JA, Hardisty, JF and Gopinath, G (eds). CRC Press, Boca Raton, FL. pp. 655-716, 2013.

Chou, FC, Chen, HY, Chen, SJ, Fang, MC, and Sytwu, HK. Rodent models for investigating the dysregulation of immune responses in type 1 diabetes. J Diabetes Res. 2013: 138412. 2013. [Medline] [CrossRef]

Coca S, Vaquero J, Escandon J, Moreno M, Peralba J, and Rodriguez J. Immunohistochemical characterization of pineocytomas. Clin Neuropathol. 11: 298-303. 1992. [Medline]

Crabtree JS, Scacheri PC, Ward JM, Garrett-Beal L, Emmert-Buck MR, Edgemon KA, Lorang D, Libutti SK, Chandrasekharappa SC, Marx SJ, Spiegel AM, and Collins FS. A mouse model of multiple endocrine neoplasia, type 1, develops multiple endocrine tumors. Proc Natl Acad Sci USA. 98: 1118-1123. 2001. [Medline] [CrossRef]

Crabtree JS, Scacheri PC, Ward JM, McNally SR, Swain GP, Montagna C, Hager JH, Hanahan D, Edlund H, Magnuson MA, Garrett-Beal L, Burns AL, Ried T, Chandrasekharappa SC, Marx SJ, Spiegel AM, and Collins FS. Of mice and MEN1: Insulinomas in a conditional mouse knockout. Mol Cell Biol. 23: 6075-6085. 2003. [Medline] [CrossRef]

Crissman JW, Valerio MG, Asiedu SA, and Evangelista-Sobel I. Ganglioneuromas of the thyroid gland in a colony of Sprague-Dawley rats. Vet Pathol. 28: 354-362. 1991. [Medline] [CrossRef]

Diehl BJ. Occurrence and regional distribution of calcareous concretions in the rat pineal gland. Cell Tissue Res. 195: 359-366. 1978. [Medline] [CrossRef]

Dill RE. The distribution of striated muscle in the epiphysis cerebri of the rat. Acta Anat (Basel). 54: 310-316. 1963. [Medline] [CrossRef]

Dillberger JE. Age-related pancreatic islet changes in Sprague-Dawley rats. Toxicol Pathol. 22: 48-55. 1994. [Medline] [CrossRef]

Doi T, Namiki M, Ashina M, Toyota N, Kokoshima H, Kanno T, Wako Y, Tayama M, Nakashima Y, Nasu M, and Tsuchitani M. Morphological and endocrinological characteristics of the endocrine systems in Wistar Hannover GALAS rats showing spontaneous dwarfs. $J$ Toxicol Pathol. 17: 197-203. 2004. [CrossRef]

Dunn TB. Normal and pathologic anatomy of the adrenal gland of the mouse, including neoplasms. J Natl Cancer Inst. 44: 1323-1389. 1970. [Medline]

Duprat P, Snell KC, and Hollander CF. Tumours of the adrenal gland. In: $P a-$ thology of tumours in laboratory animals. Volume 1. Tumours of the rat. Turusov, VS and Mohr, U (eds). $2^{\text {nd }}$ ed. International Agency for Research on Cancer; Distributed in the USA by Oxford University Press, Lyon, France; New York. pp. 573-596, 1990.

Ehses JA, Perren A, Eppler E, Ribaux P, Pospisilik JA, Maor-Cahn R, Gueripel X, Ellingsgaard H, Schneider MK, Biollaz G, Fontana A, Reinecke M, Homo-Delarche F, and Donath MY. Increased number of islet-associated macrophages in type 2 diabetes. Diabetes. 56: 2356-2370. 2007. [Medline] [CrossRef]

Elmore S. Apoptosis: a review of programmed cell death. Toxicol Pathol. 35 495-516. 2007. [Medline] [CrossRef]

Elmore SA, Berridge BR, Boyle MC, Cora MC, Hoenerhoff MJ, Kooistra L, Laast VA, Morrison JP, Rao D, Rinke M, and Yoshizawa K. Proceedings of the 2012 National Toxicology Program Satellite Symposium. Toxicol Pathol. 41: 151-180. 2013. [Medline] [CrossRef]
Elmore SA, Dixon D, Hailey JR, Harada T, Herbert RA, Maronpot RR, Nolte T, Rehg JE, Rittinghausen S, Rosol TJ, Satoh H, Vidal JD, Willard-Mack CL, and Creasy DM. Recommnedations from the INHAND apoptosis/ necrosis working group. Toxicol Pathol. 44: 173-188. 2016. [Medline] [CrossRef]

Engeland WC, and Levay-Young BK. Changes in the glomerulosa cell phenotype during adrenal regeneration in rats. Am J Physiol. 276: R1374R1382. 1999. [Medline]

Everds NE, Snyder PW, Bailey KL, Bolon B, Creasy DM, Foley GL, Rosol TJ, and Sellers T. Interpreting stress responses during routine toxicity studies: a review of the biology, impact, and assessment. Toxicol Pathol. 41: 560-614. 2013. [Medline] [CrossRef]

Faccini JM, Abbott DP, and Paulus GJJ. Endocrine Glands. In: Mouse histopathology: A glossary for use in toxicity and carcinogenicity studies. Elsevier, Amsterdam; New York. pp. 169-186, 1990.

Figarella-Branger D, Dufour H, Fernandez C, Bouvier-Labit C, Grisoli F, and Pellissier JF. Pituicytomas, a mis-diagnosed benign tumor of the neurohypophysis: report of three cases. Acta Neuropathol. 104: 313-319. 2002. [Medline]

Fitzgerald JE, Schardein JL, and Kaump DH. Several uncommon pituitary tumors in the rat. Lab Anim Sci. 21: 581-584. 1971. [Medline]

Fitzgerald JE, Schardein JL, and Kurtz SM. Spontaneous tumors of the nervous system in albino rats. $J$ Natl Cancer Inst. 52: 265-273. 1974. [Medline] [CrossRef]

Fraser $\mathrm{H}$. Brain tumours in mice, with particular reference to astrocytoma. Food Chem Toxicol. 24: 105-111. 1986. [Medline] [CrossRef]

Frith CH. Histology, Adrenal Gland, Mouse. In: Monographs on pathology of laboratory animals. Endocrine system. Jones, TC, Capen, CC and Mohr, U (eds). $2^{\text {nd }}$ ed. Springer, Berlin; New York. pp. 386-391, 1996a.

Frith CH. Lipogenic Pigmentation, Adrenal Cortex, Mouse. In: Monographs on pathology of laboratory animals. Endocrine system. Jones, TC, Capen, CC and Mohr, U (eds). $2^{\text {nd }}$ ed. Springer, Berlin; New York. pp. 458-462, 1996b.

Frith CH, Botts S, Jokinen MP, Eighmy JJ, Hailey JR, Morgan SJ, and Chandra M. Non-Proliferative Lesions of the Endocrine System in Rats. In: Guides for Toxicologic Pathology. STP/ARP/AFIP, Washington, DC. pp. $1-22,2000$.

Frith $\mathrm{CH}$, and Chandra M. Incidence, distribution, and morphology of amyloidosis in Charles Rivers CD-1 mice. Toxicol Pathol. 19: 123-127. 1991. [Medline] [CrossRef]

Frith CH, and Dunn TB. Tumors of the adrenal gland. In: Pathology of tumours in laboratory animals. Volume 2. Tumours of the mouse. Turusov, VS and Mohr, U (eds). 2 $2^{\text {nd }}$ ed. International Agency for Research on Cancer; Distributed in the USA by Oxford University Press, Lyon, France; New York. pp. 595-609, 1994.

Frith CH, and Fetters J. Ectopic Parathyroid, Mouse. In: Monographs on pathology of laboratory animals. Endocrine system. Jones, TC, Capen, CC and Mohr, U (eds). $2^{\text {nd }}$ ed. Springer, Berlin; Heidelberg; New York. pp. 333-334, 1996a.

Frith CH, and Fetters J. Ectopic Thyroid, Mouse. In: Monographs on pathology of laboratory animals. Endocrine system. Jones, TC, Capen, CC and Mohr, U (eds). $2^{\text {nd }}$ ed. Springer, Berlin; New York. pp. 239-240, 1996b.

Frith $\mathrm{CH}$, and Heath JE. Morphological classification and incidence of thyroid tumors in untreated aged mice. J Gerontol. 39: 7-10. 1984. [Medline] [CrossRef]

Frith CH, and Sheldon WD. Hyperplasia, Adenoma, and Carcinoma of Pancreatic Islets, Mouse. In: Monographs on pathology of laboratory animals. Endocrine system. Jones, TC, Capen, CC and Mohr, U (eds). $2^{\text {nd }}$ ed. Springer, Berlin; New York. pp. 361-367, 1996.

Frith CH, and Ward JM. Digestive System. In: Color atlas of neoplastic and non-neoplastic lesions in aging mice. Elsevier, Amsterdam; New York. pp. 7-14, 1988a.

Frith CH, and Ward JM. Endocrine System. In: Color atlas of neoplastic and non-neoplastic lesions in aging mice. Elsevier, Amsterdam; New York. pp. 33-37, 1988 b.

Furukawa S, Kobayashi K, Usuda K, Tamura T, Miyamoto Y, Hayashi K, Ikeyama S, Goryo M, and Okada K. Spontaneous pinealoma in a male 
Crj:CD (SD) IGS rat. J Vet Med Sci. 61: 41-44. 1999. [Medline] [CrossRef]

Galluzzi L, Vitale I, Abrams JM, Alnemri ES, Baehrecke EH, Blagosklonny MV, Dawson TM, Dawson VL, El-Deiry WS, Fulda S, Gottlieb E, Green DR, Hengartner MO, Kepp O, Knight RA, Kumar S, Lipton SA, Lu X, Madeo F, Malorni W, Mehlen P, Nuñez G, Peter ME, Piacentini M, Rubinsztein DC, Shi Y, Simon HU, Vandenabeele P, White E, Yuan J, Zhivotovsky B, Melino G, and Kroemer G. Molecular definitions of cell death subroutines: recommendations of the Nomenclature Committee on Cell Death 2012. Cell Death Differ. 19: 107-120. 2012. [Medline] [CrossRef]

Glaister JR, Samuels DM, and Tucker MJ. Ganglioneuroma-containing tumours of the adrenal medulla in Alderly-Park rats. Lab Anim. 11: 35-37. 1977. [Medline] [CrossRef]

Goodman DG. Subcapsular-Cell Hyperplasia, Adrenal, Mouse. In: Monographs on pathology of laboratory animals. Endocrine system. Jones, TC, Capen, CC and Mohr, U (eds). $2^{\text {nd }}$ ed. Springer, Berlin; New York. pp. 464-467, 1996.

Gopinath C, and Mowat V. The Endocrine System. In: Atlas of toxicological pathology. Springer. pp. 169-195, 2014.

Götz W, Theuring F, Schachenmayr W, and Korf HW. Midline brain tumors in MSV-SV 40-transgenic mice originate from the pineal organ. Acta Neuropathol. 83: 308-314. 1992. [Medline] [CrossRef]

Greaves P. Adrenal Gland. In: Histopathology of preclinical toxicity studies: Interpretation and relevance in drug safety studies. $4^{\text {th }}$ ed. Elsevier/AP, Amsterdam; Boston. pp. 740-761, 2012a.

Greaves P. Endocrine Pancreas. In: Histopathology of preclinical toxicity studies: Interpretation and relevance in drug safety studies. $4^{\text {th }}$ ed. Elsevier/AP, Amsterdam; Boston. pp. 501-535, 2012b.

Greaves P. Pituitary Gland. In: Histopathology of preclinical toxicity studies: Interpretation and relevance in drug safety studies. $4^{\text {th }}$ ed. Elsevier/AP, Amsterdam; Boston. pp. 727-740, 2012c.

Greaves P. Thyroid Gland. In: Histopathology of preclinical toxicity studies: Interpretation and relevance in drug safety studies. $4^{\text {th }}$ ed. Elsevier/AP, Amsterdam; Boston. pp. 761-779, 2012d.

Greaves P, and Faccini JM. Endocrine Glands. In: Rat histopathology: A glossary for use in toxicity and carcinogenicity studies. $2^{\text {nd }}$ ed. Elsevier, Amsterdam; New York. pp. 222-257, 1992.

Günther T, Chen ZF, Kim J, Priemel M, Rueger JM, Amling M, Moseley JM, Martin TJ, Anderson DJ, and Karsenty G. Genetic ablation of parathyroid glands reveals another source of parathyroid hormone. Nature. 406: 199-203. 2000. [Medline] [CrossRef]

Hamlin MH II, and Banas DA. Adrenal Gland. In: Pathology of the Fischer rat : reference and atlas. Academic Press, San Diego. pp. 501-518., 1990.

Hardisty JF, and Boorman GA. Thyroid Gland. In: Pathology of the Fischer Rat: Reference and Atlas. Boorman, GA, Eustis, SL, Elwell, MR and MacKenzie, WF (eds). Academic Press, San Diego. pp. 519-536, 1990.

Hardisty JF, and Boorman GA. Thyroid and Parathyroid Gland. In: Pathology of the Mouse. Maronpot, RR, Boorman, GA and Gaul, BW (eds). Cache River Press, Vienna, Illinois. pp. 537-554, 1999.

Harvey PW, and Sutcliffe C. Adrenocortical hypertrophy: establishing cause and toxicological significance. J Appl Toxicol. 30: 617-626. 2010. [Medline] [CrossRef]

Heath JE. Adenoma and Carcinoma, Adrenal Cortex, Mouse. In: Monographs on pathology of laboratory animals. Endocrine system. Jones, TC, Capen, CC and Mohr, U (eds). $2^{\text {nd }}$ ed. Springer, Berlin; New York. pp. $448-454,1996$ a.

Heath JE. Adenoma and Carcinoma, Thyroid Follicular Cell, Mouse. In: Monographs on pathology of laboratory animals. Endocrine system. Jones, TC, Capen, CC and Mohr, U (eds). $2^{\text {nd }}$ ed. Springer, Berlin; New York. pp. 254-261, 1996b.

Heath JE. Adenoma, and Carcinoma of Pancreatic Islets, Rat. In: Monographs on pathology of laboratory animals. Endocrine system. Jones, TC, Capen, CC and Mohr, U (eds). $2^{\text {nd }}$ ed. Springer, Berlin; New York. pp. 371-378, 1996c.

Heath JE. Gangliocytoma, Pituitary Gland, Rat. In: Monographs on pathology of laboratory animals. Endocrine system. Jones, TC, Capen, CC and Mohr, U (eds). 2nd ed. Springer, Berlin; New York. pp. 98-101, 1996d.
Heath JE, and Winokur TS. Case report: pineocytoma in a male Fischer 344 rat. Toxicol Pathol. 26: 294-297. 1998. [Medline] [CrossRef]

Heider K. Spontaneous craniopharyngioma in a mouse. Vet Pathol. 23: 522523. 1986. [Medline] [CrossRef]

Hershkovitz L, Beuschlein F, Klammer S, Krup M, and Weinstein Y. Adrenal 20alpha-hydroxysteroid dehydrogenase in the mouse catabolizes progesterone and 11-deoxycorticosterone and is restricted to the X-zone. Endocrinology. 148: 976-988. 2007. [Medline] [CrossRef]

Hill GD, Pace V, Persohn E, Bresser C, Haseman JK, Tischler AS, and Nyska A. A comparative immunohistochemical study of spontaneous and chemically induced pheochromocytomas in B6C3F1 mice. Endocr Pathol. 14: 81-91. 2003. [Medline] [CrossRef]

Hirato J, and Nakazato Y. Pathology of pineal region tumors. J Neurooncol. 54: 239-249. 2001. [Medline] [CrossRef]

Hoenerhoff MJ, Hill GD, and Gruebbel MM. Adrenal Gland - Mineralization. In: NTP Nonneoplastic Lesion Atlas. National Toxicology Program. http://ntp.niehs.nih.gov/nnl/endocrine/adrenal/mineral/adrenal-glandmineralization-pdf_508.pdf. pp. 1-2 accessed February 09, 2016.

Höppener JW, Oosterwijk C, van Hulst KL, Verbeek JS, Capel PJ, de Koning EJ, Clark A, Jansz HS, and Lips CJ. Molecular physiology of the islet amyloid polypeptide (IAPP)/amylin gene in man, rat, and transgenic mice. $J$ Cell Biochem. 55(Suppl): 39-53. 1994. [Medline] [CrossRef]

Hosokawa S, Fukuta T, Imai T, Sonoda J, Aoki T, Hayakawa K, Motooka S, and Nakanowatari J. Pituitary carcinoma of pars distalis as a common neoplasm in Fischer-344 rats. Toxicol Pathol. 21: 283-287. 1993. [Medline] [CrossRef]

Huggins C, and Morii S. Selective adrenal necrosis and apoplexy induced by 7, 12-dimethylbenz(a)anthracene. J Exp Med. 114: 741-760. 1961. [Medline] [CrossRef]

Humbert W, and Pévet P. The pineal gland of the aging rat: calcium localization and variation in the number of pinealocytes. J Pineal Res. 18: 32-40. 1995. [Medline] [CrossRef]

Imaoka M, Kato M, Tago S, Gotoh M, Satoh H, and Manabe S. Effects of estradiol treatment and/or ovariectomy on spontaneous hemorrhagic lesions in the pancreatic islets of Sprague-Dawley rats. Toxicol Pathol. 37: 218-226. 2009. [Medline] [CrossRef]

Imaoka M, Satoh H, and Furuhama K. Age- and sex-related differences in spontaneous hemorrhage and fibrosis of the pancreatic islets in SpragueDawley rats. Toxicol Pathol. 35: 388-394. 2007. [Medline] [CrossRef]

Imazawa T, Nishikawa A, Shibutani M, Ogasawara H, Furukawa F, Ikeda T, Suda K, and Hirose M. Induction of pancreatic islet cell tumors in rats by repeated intravenous administration of 4-hydroxyaminoquinoline 1-oxide. Toxicol Pathol. 29: 320-327. 2001. [Medline] [CrossRef]

Inoue S, and Kisilevsky R. A high resolution ultrastructural study of experimental murine AA amyloid. Lab Invest. 74: 670-683. 1996. [Medline]

Iwata H, Hosoi M, Miyajima R, Yamamoto S, Mikami S, Yamakawa S, Enomoto M, Imazawa T, and Mitsumori K. Morphogenesis of craniopharyngeal derivatives in the neurohypophysis of Fisher 344 rats: abnormally developed epithelial tissues including parotid glands derived from the stomatodeum. Toxicol Pathol. 28: 568-574. 2000. [Medline] [CrossRef]

Jameson JL, Weiss J, Polak JM, Childs GV, Bloom SR, Steel JH, Capen CC, Prentice DE, Fetter AW, and Langloss JM. Glycoprotein hormone alphasubunit-producing pituitary adenomas in rats treated for one year with calcitonin. Am J Pathol. 140: 75-84. 1992. [Medline]

Janat MF, and Shire JG. The adrenal X-zone of mice: genetic analysis of its development with recombinant-inbred strains. Exp Biol. 46: 217-221. 1987. [Medline]

Johnson KH, OBrien TD, Betsholtz C, and Westermark P. Islet amyloid polypeptide: mechanisms of amyloidogenesis in the pancreatic islets and potential roles in diabetes mellitus. Lab Invest. 66: 522-535. 1992. [Medline]

Jokinen MP, and Botts S. Tumors of the thyroid gland. In: Pathology of tumours in laboratory animals. Volume 2. Tumours of the mouse. Turusov, VS and Mohr, U (eds). $2^{\text {nd }}$ ed. International Agency for Research on Cancer (distributed in the USA by Oxford University Press), Lyon; New York. pp. 565-593, 1994.

Jones IC. The disappearance of the $\mathrm{X}$ zone of the mouse adrenal cortex during 
first pregnancy. Proc R Soc Lond B Biol Sci. 139: 398-410. 1952. [Medline] [CrossRef]

Jones TC, Hunt RD, and King NW. (1997). Endocrine Glands. In, Veterinary Pathology. Jones, TC, Hunt, RD and King, NW (eds). $6^{\text {th }}$ ed. Williams \& Wilkins, Baltimore, MD. pp. 1223-1257, 1997.

Jouvet A, Fauchon F, Bouffet E, Saint-Pierre G, Champier J, and Ferre Montange M. Tumors of the pineal parenchymal and glial cells. In: Russell and Rubinstein's pathology of tumors of the nervous system. Bigner, DD, McLendon, RE and Bruner, JM (eds). $7^{\text {th }}$ ed. Hodder Arnold (distributed in USA by Oxford University Press), London; New York. pp. 413-425, 2006.

Kalter H. Sporadic congenital malformations of newborn inbred mice. Teratology. 1: 193-199. 1968. [Medline] [CrossRef]

Kamino K, Kittel B, and Ernst H. Adenoma, Carcinoma, Parathyroid, Rat. In: Monographs on pathology of laboratory animals. Endocrine system. Jones, TC, Capen, CC and Mohr, U (eds). 2nd ed. Springer, Berlin; New York. pp. 350-358, 1996a.

Kamino K, Kittel B, and Ernst H. Hyperplasia, Parathyroid, Rat. In: Monographs on pathology of laboratory animals. Endocrine system. Jones, TC, Capen, CC and Mohr, U (eds). $2^{\text {nd }}$ ed. Springer, Berlin; New York. pp. 338-345, 1996b.

Kanno J, Nemoto T, Kasuga T, and Hayashi Y. Effects of a six-week exposure to excess iodide on thyroid glands of growing and nongrowing male Fischer-344 rats. Toxicol Pathol. 22: 23-28. 1994. [Medline] [CrossRef]

Kanno J, Onodera H, Furuta K, Maekawa A, Kasuga T, and Hayashi Y. Tumor-promoting effects of both iodine deficiency and iodine excess in the rat thyroid. Toxicol Pathol. 20: 226-235. 1992. [Medline] [CrossRef]

Karbe E, and Ernst H. Craniopharyngeal Derivates in teh Neurohypophysis, Rat and Hamster. In: Monographs on pathology of laboratory animals. Endocrine system. Jones, TC, Capen, CC and Mohr, U (eds). $2^{\text {nd }}$ ed. Springer, Berlin; New York. pp. 114-118, 1996.

Kaspareit-Rittinghausen J, Rapp K, Deerberg F, Wcislo A, and Messow C. Hereditary polycystic kidney disease associated with osteorenal syndrome in rats. Vet Pathol. 26: 195-201. 1989. [Medline] [CrossRef]

Kaspareit-Rittinghausen J, Wiese K, Deerberg F, and Nitsche B. Incidence and morphology of spontaneous thyroid tumours in different strains of rats. J Comp Pathol. 102: 421-432. 1990. [Medline] [CrossRef]

Kaspareit J, and Rittinghausen S. Spontaneous neoplastic lesions in Harlan Sprague-Dawley rats. Exp Toxicol Pathol. 51: 105-107. 1999. [Medline] [CrossRef]

Kittel B, Ernst H, and Kamino K. Anatomy, Histology, and Ultrastructure, Parathyroid, Mouse. In: Monographs on pathology of laboratory animals. Endocrine system. Jones, TC, Capen, CC and Mohr, U (eds). $2^{\text {nd }}$ ed. Springer, Berlin; New York. pp. 328-330, 1996a.

Kittel B, Ernst H, and Kamino K. Anatomy, Histology, and Ultrastructure, Parathyroid, Rat. In: Monographs on pathology of laboratory animals. Endocrine system. Jones, TC, Capen, CC and Mohr, U (eds). $2^{\text {nd }}$ ed. Springer, Berlin, New York. pp. 330-333, 1996b.

Kiupel M, Capen CC, Miller M, and Smedley R. C-cell hyperplasia. In: Histological Classification of Tumors of the Endocrine System of Domestic Animals. Armed Forces Institute of Pathology (USA), Washington DC. pp. 38-39, 2008a.

Kiupel M, Capen CC, Miller M, and Smedley R. Tumors of the adrenal gland. In: Histological Classification of Tumors of the Endocrine System of Domestic Animals. Armed Forces Institute of Pathology (USA), Washington, DC. pp. 43-49, 2008 b.

Koestner A, and Solleveld HA. Tumors of the Pineal Gland, Rat. In: Monographs on pathology of laboratory animals. Endocrine system. Jones, TC, Capen, CC and Mohr, U (eds). $2^{\text {nd }}$ ed. Springer, Berlin; New York. pp. 205-213, 1996.

Koivisto C, Flake GP, Kolenda-Roberts H, Masinde T, Kissling GE, Sills RC, and Hoenerhoff MJ. Immunohistochemical investigation of F344/ $\mathrm{N}$ rat islet cell tumors from national toxicology program studies. Toxicol Pathol. 40: 751-763. 2012. [Medline] [CrossRef]

Kokoshima H, Doi T, Yamada N, and Tsuchitani M. Proliferative lesions in thyroid follicular cells of dwarfs derived from Wistar Hannover GALAS rats. Toxicol Pathol. 42: 565-572. 2014. [Medline] [CrossRef]
Korf HW, Götz W, Herken R, Theuring F, Gruss P, and Schachenmayr W. $\mathrm{S}$-antigen and rod-opsin immunoreactions in midline brain neoplasms of transgenic mice: similarities to pineal cell tumors and certain medulloblastomas in man. J Neuropathol Exp Neurol. 49: 424-437. 1990. [Medline] [CrossRef]

Krinke G, Naylor DC, Schmid S, Fröhlich E, and Schnider K. The incidence of naturally-occurring primary brain tumours in the laboratory rat. $J$ Comp Pathol. 95: 175-192. 1985. [Medline] [CrossRef]

Krinke GJ, Kaufmann W, Mahrous AT, and Schaetti P. Morphologic characterization of spontaneous nervous system tumors in mice and rats. Toxicol Pathol. 28: 178-192. 2000. [Medline] [CrossRef]

Kroemer G, Galluzzi L, Vandenabeele P, Abrams J, Alnemri ES, Baehrecke EH, Blagosklonny MV, El-Deiry WS, Golstein P, Green DR, Hengartner M, Knight RA, Kumar S, Lipton SA, Malorni W, Nuñez G, Peter ME, Tschopp J, Yuan J, Piacentini M, Zhivotovsky B, Melino G. Nomenclature Committee on Cell Death 2009. Classification of cell death: recommendations of the Nomenclature Committee on Cell Death 2009. Cell Death Differ. 16: 3-11. 2009. [Medline] [CrossRef]

Krstić R. Electron microscopic study of the striated muscle fibers in the pineal body of Wistar rats. Z Zellforsch Mikrosk Anat. 128: 227-240. 1972; (in German). [Medline]

Kuchelmeister K, von Borcke IM, Klein H, Bergmann M, and Gullotta F. Pleomorphic pineocytoma with extensive neuronal differentiation: report of two cases. Acta Neuropathol. 88: 448-453. 1994. [Medline] [CrossRef]

Laast VA, Larsen T, Allison N, Hoenerhoff MJ, and Boorman GA. Distinguishing cystic degeneration from other aging lesions in the adrenal cortex of Sprague-Dawley rats. Toxicol Pathol. 42: 823-829. 2014. [Medline] [CrossRef]

Landes C, Krinke G, and Zak F. Lipid Hyperplasia, Adrenal Cortex, Rat. In: Monographs on pathology of laboratory animals. Endocrine system. Jones, TC, Capen, CC and Mohr, U (eds). $2^{\text {nd }}$ ed. Springer, Berlin; New York. pp. 481-486, 1996.

Laroque P, Duprat P, and Hollander CF. Neoplasia and preneoplasia of the adrenal glands. In: Pathology of Neoplasia and Preneoplasia in Rodents. EULEP Color Atlas. Bannasch, P and Goessner, W (eds). Schattauer, Stuttgard, New York. pp. 129-140, 1997.

Lee AK, DeLellis RA, Blount M, Nunnemacher G, and Wolfe HJ. Pituitary proliferative lesions in aging male Long-Evans rats. A model of mixed multiple endocrine neoplasia syndrome. Lab Invest. 47: 595-602. 1982. [Medline]

Leiter EH, and Herbert L. Aging, Pancreatic Islets, and Glucose Homeostasis in Inbred Mice. In: Pathobiology of the Aging Mouse. Mohr, U, Dungworth, DL, Capen, CC, Carlton, WW, Sundberg, JP and Ward, JM (eds). ILSI Press, Washington, D.C. pp. 153-170, 1996.

Levin S, Bucci TJ, Cohen SM, Fix AS, Hardisty JF, LeGrand EK, Maronpot RR, and Trump BF. The nomenclature of cell death: recommendations of an ad hoc Committee of the Society of Toxicologic Pathologists. Toxicol Pathol. 27: 484-490. 1999. [Medline] [CrossRef]

Lewis DJ, and Cherry CP. Parathyroid adenoma in mice: a report of two cases. J Comp Pathol. 92: 337-339. 1982. [Medline] [CrossRef]

Libutti SK, Crabtree JS, Lorang D, Burns AL, Mazzanti C, Hewitt SM, OConnor S, Ward JM, Emmert-Buck MR, Remaley A, Miller M, Turner E, Alexander HR, Arnold A, Marx SJ, Collins FS, and Spiegel AM. Parathyroid gland-specific deletion of the mouse Men1 gene results in parathyroid neoplasia and hypercalcemic hyperparathyroidism. Cancer Res. 63: 8022-8028. 2003. [Medline]

Liebelt AG. Tumors of the pituitary gland. In: Pathology of tumours in laboratory animals. Volume 2. Tumours of the mouse. Turusov, VS and Mohr, $\mathrm{U}$ (eds). $2^{\text {nd }}$ ed. International Agency for Research on Cancer; Oxford University Press, Lyon, France; New York. pp. 527-563, 1994.

Lloyd RV. Tumours of the pituitary gland. In: Pathology of tumours in laboratory animals. Volume 1. Tumours of the rat. Turusov, VS and Mohr, U (eds). $2^{\text {nd }}$ ed. International Agency for Research on Cancer; Distributed in the USA by Oxford University Press, Lyon, France; New York. pp. 499-537, 1990.

Lloyd RV, Kawashima A, and Tischler AS. Adrenal soft tissue and germ 
cell tumors. In: Pathology and genetics of tumours of endocrine organs. World Health Organization classification of tumours. DeLellis, RA (ed) IARC Press, Lyon. pp. 169, 2004.

Longeart LE. Adrenal Medullary Tumors, Mouse. In: Monographs on pathology of laboratory animals. Endocrine system. Jones, TC, Capen, CC and Mohr, U (eds). 2nd ed. Springer, Berlin; New York. pp. 421-427, 1996.

Longnecker DS, and Millar PM. Tumours of the pancreas. In: Pathology of tumours in laboratory animals. Volume 1. Tumours of the rat. Turusov, VS and Mohr, U (eds). $2^{\text {nd }}$ ed. International Agency for Research on Cancer; Distributed in the USA by Oxford University Press, Lyon, France; New York. pp. 241-257, 1990.

Louis DN, Ohgaki H, Wiestler OD, Cavenee WK, Burger PC, Jouvet A, Scheithauer BW, and Kleihues P. The 2007 WHO classification of tumours of the central nervous system. Acta Neuropathol. 114: 97-109. 2007. [Medline] [CrossRef]

MacKenzie WF, and Boorman GA. Pituitary Gland. In: Pathology of the Fischer rat: Reference and atlas. Academic Press, San Diego. pp. 485500, 1990.

Maekawa A, Onodera H, Tanigawa H, Furuta K, Takahashi M, Kurokawa Y, Kokubo T, Ogiu T, Uchida O, Kobayashi K, and Hayaski Y. Spontaneous tumors of the nervous system and associated organs and/or tissues in rats. Gan. 75: 784-791. 1984. [Medline]

Mahler JF, and Elwell MR. Pituitary Gland. In: Pathology of the Mouse. Maronpot, RR, Boorman, GA and Gaul, BW (eds). Cache River Press, Vienna, Illinois, USA. pp. 491-507, 1999.

Maita K, Hirano M, Harada T, Mitsumori K, Yoshida A, Takahashi K, Nakashima N, Kitazawa T, Enomoto A, Inui K, and Shirasu Y. Mortality, major cause of moribundity, and spontaneous tumors in CD-1 mice. Toxicol Pathol. 16: 340-349. 1988. [Medline] [CrossRef]

Majeed SK. Survey on spontaneous systemic amyloidosis in aging mice. Arzneimittelforschung. 43: 170-178. 1993. [Medline]

Majeed SK, and Harling SM. Malignant phaeochromocytoma with widespread metastasis in the rat. J Comp Pathol. 96: 575-580. 1986. [Medline] [CrossRef]

Majka JA, Solleveld HA, Barthel CH, and Van Zwieten MJ. Proliferative Lesions of the Pituitary in Rats. In: Guides for Toxicologic Pathology. STP/ ARP/AFIP, Washington, DC. pp. 1-8, 1990.

Maronpot RR, Yoshizawa K, Nyska A, Harada T, Flake G, Mueller G, Singh B, and Ward JM. Hepatic enzyme induction: histopathology. Toxicol Pathol. 38: 776-795. 2010. [Medline] [CrossRef]

Martín-Lacave I, Rojas F, Bernabé R, Utrilla JC, Fernández-Santos JM, De Miguel M, and Conde E. Comparative immunohistochemical study of normal, hyperplastic and neoplastic $\mathrm{C}$ cells of the rat thyroid gland. Cell Tissue Res. 309: 361-368. 2002. [Medline] [CrossRef]

Martinez MJ, and Mog SR. Spontaneous complex pheochromocytoma in a Fischer 344 rat. Vet Pathol. 38: 470-473. 2001. [Medline] [CrossRef]

McComb DJ, Kovacs K, Beri J, Zak F, Milligan JV, and Shin SH. Pituitary gonadotroph adenomas in old Sprague-Dawley rats. J Submicrosc Cytol. 17: 517-530. 1985. [Medline]

McInnes EF. Wistar and Sprague-Dawley rats. In: Background Lesions in Laboratory Animals. Saunders Elsevier, Edinburgh. pp. 17-36, 2012.

Mense MG, and Rosol TJ. Endocrine Pancreas. In: Boorman's Pathology of the Rat. Suttie, AW, Leininger, JR and Bradley, AE (eds). $2^{\text {nd }}$ ed. Academic Press, London; San Diego; Cambridge, MA; Oxford. pp. 1-10, 2018a.

Mense MG, and Rosol TJ. Parathyroid Gland. In: Boorman's Pathology of the Rat. Suttie, AW, Leininger, JR and Bradley, AE (eds). $2^{\text {nd }}$ ed. Academic Press, London; San Diego; Cambridge, MA; Oxford. pp. 1-7, $2018 \mathrm{~b}$.

Molenaar WM, Lee VM, and Trojanowski JQ. Early fetal acquisition of the chromaffin and neuronal immunophenotype by human adrenal medullary cells. An immunohistological study using monoclonal antibodies to chromogranin A, synaptophysin, tyrosine hydroxylase, and neuronal cytoskeletal proteins. Exp Neurol. 108: 1-9. 1990. [Medline] [CrossRef]

Moroki T, Sasaki T, Yoshizawa K, and Doi T. A spontaneously occurring malignant pituicytoma in a male sprague dawley rat. J Toxicol Pathol. 28: 171-176. 2015. [Medline] [CrossRef]

Moroki T, Yoshikawa Y, Yoshizawa K, Tsubura A, and Yasui H. Morphological characterization of systemic changes in KK-Ay mice as an animal model of type 2 diabetes. In Vivo. 27: 465-472. 2013. [Medline]

Murthy ASK. Neoplasms of the thyroid gland. In: Atlas of tumor pathology of the Fischer rat. Stinson, SF, Schuller, HM and Reznik, G (eds). CRC Press, Boca Raton, FL. pp. 323-365, 1990.

Murugaiyan P, and Salm AK. Dehydration-induced proliferation of identified pituicytes in fully adult rats. Glia. 15: 65-76. 1995. [Medline] [CrossRef]

Naeser P. Structure of the adrenal glands in mice with the obese-hyperglycaemic syndrome (gene symbol ob). Acta Pathol Microbiol Scand [A]. 83: 120-126. 1975. [Medline]

Napalkov NP. Tumours of the thyroid gland. In: Pathology of tumours in laboratory animals. Volume 1. Tumours of the rat. Turusov, VS and Mohr, U (eds). $2^{\text {nd }}$ ed. International Agency for Research on Cancer; Distributed in the USA by Oxford University Press, Lyon; New York. pp. 539-572, 1990.

Neville AM, and O'Hare MJ. Myelolipoma and related changes. In: The human adrenal cortex : pathology and biology, an integrated approach. Springer-Verlag, Berlin; New York. pp. 290-293, 1982.

Nugent DA, Smith DM, and Jones HB. A review of islet of Langerhans degeneration in rodent models of type 2 diabetes. Toxicol Pathol. 36: 529-551. 2008. [Medline] [CrossRef]

Nyska A, and Maronpot RR. Adrenal Gland. In: Pathology of the Mouse. Maronpot, RR, Boorman, G.A., Gaul, B.W. (ed) Cache River Press, Vienna, Illinois, USA. pp. 509-536, 1999.

Ohshima M, and Ward JM. Dietary iodine deficiency as a tumor promoter and carcinogen in male F344/NCr rats. Cancer Res. 46: 877-883. 1986. [Medline]

Oishi Y, Matsumoto M, Yoshizawa K, Fujihiro S, Tsubura A, and Morii S. Spontaneous pituitary adenomas of the pars intermedia in mice and rats: Histopathological and immunohistochemical studies. J Toxicol Pathol. 5: 223-231. 1992. [CrossRef]

Okazaki Y, Katsuta O, Yokoyama M, Wako Y, Yamagishi Y, and Tsuchitani M. Gangliocytoma with immature neuronal cell elements in the pituitary of a rat. J Vet Med Sci. 59: 833-836. 1997. [Medline] [CrossRef]

Osamura RY. Histochemical identification of hormones in pituitary tumors, rat. In: Monographs on Pathology of Laboratory Animals. Endocrine System. Jones, TC, Capen, CC and Mohr, U (eds). $2^{\text {nd }}$ ed. Springer, Berlin; New York. pp. 66-70, 1996a.

Osamura RY. Histology, Ultrastructure, and Immunohistochemistry, Pituitary Gland, Rat. In: Monographs on Pathology of Laboratory Animals. Endocrine system. Jones, TC, Capen, CC and Mohr, U (eds). $2^{\text {nd }}$ ed. Springer, Berlin; New York. pp. 33-41, 1996 b.

Osamura RY. Pituitary tumors induced by estrogen, rat. In: Monographs on Pathology of Laboratory Animals. Endocrine system. Jones, TC, Capen, CC and Mohr, U (eds). $2^{\text {nd }}$ ed. Springer, Berlin; New York. pp. 90-94, $1996 \mathrm{c}$.

Pace V, Heider K, Persohn E, and Schaetti P. Spontaneous malignant craniopharyngioma in an albino rat. Vet Pathol. 34: 146-149. 1997. [Medline] [CrossRef]

Pace V, and Perentes E. Mixed pituitary adenoma-gangliocytoma in a female albino rat. Acta Neuropathol. 101: 277-280. 2001. [Medline]

Pace V, Perentes E, and Germann PG. Pheochromocytomas and ganglioneuromas in the aging rats: morphological and immunohistochemical characterization. Toxicol Pathol. 30: 492-500. 2002. [Medline] [CrossRef]

Pace V, Scarsella S, and Perentes E. Parathyroid gland carcinoma in a Wistar rat. Vet Pathol. 40: 203-206. 2003. [Medline] [CrossRef]

Parker GA, and Valerio MG. Accessory Adrenocortical Tissue, Rat. In: Monographs on pathology of laboratory animals. Endocrine system. Jones, TC, Capen, CC and Mohr, U (eds). $2^{\text {nd }}$ ed. Springer, Berlin; New York. pp. 394-396, 1996a.

Parker GA, and Valerio MG. Ectopic Thymus, Thyroid, Rat. In: Monographs on pathology of laboratory animals. Endocrine system. Jones, TC, Capen, CC and Mohr, U (eds). 2nd ed. Springer, Berlin; New York. pp. 241-243, 1996b.

Parker GA, and Valerio MG. Ectopic Thyroid, Rat. In: Monographs on pathology of laboratory animals. Endocrine system. Jones, TC, Capen, CC and Mohr, U (eds). $2^{\text {nd }}$ ed. Springer, Berlin; New York. pp. 240-241, 1996c.

Parker GA, and Valerio MG. Lipogenic Pigmentation, Adrenal Cortex, Rat. In: Monographs on pathology of laboratory animals. Endocrine system. 
Jones, TC, Capen, CC and Mohr, U (eds). 2ed ed. Springer, Berlin; New York. pp. 462-464, 1996d.

Patterson DR, Hamlin MH II, Hottendorf GH, Gough A, and Brown WR. Proliferative Lesions of the Adrenal Glands in Rats. In: Guides for Toxicologic Pathology. STP/ARP/AFIP, Washington, DC. pp. 1-12, 1995.

Pilling AM, Jones SA, Endersby-Wood HJ, McCormack NA, and Turton JA. Expression of thyroglobulin and calcitonin in spontaneous thyroid gland tumors in the Han Wistar rat. Toxicol Pathol. 35: 348-355. 2007. [Medline] [CrossRef]

Price SC, Chescoe D, Grasso P, Wright M, and Hinton RH. Alterations in the thyroids of rats treated for long periods with di-(2-ethylhexyl) phthalate or with hypolipidaemic agents. Toxicol Lett. 40: 37-46. 1988. [Medline] [CrossRef]

Prosenc N, and Cervós-Navarro J. Ultrastructural morphology of the aged pineal. Ann NY Acad Sci. 719: 64-76. 1994. [Medline] [CrossRef]

Quintanar-Stephano A, Muñoz Fernández L, Quintanar JL, and Kovacs K. Cysts in the rat adenohypophysis: incidence and histology. Endocr Pathol. 12: 63-71. 2001. [Medline] [CrossRef]

Rao-Rupanagudi S, Heywood R, and Gopinath C. Age-related changes in thyroid structure and function in Sprague-Dawley rats. Vet Pathol. 29: 278-287. 1992. [Medline] [CrossRef]

Reaven EP, and Reaven GM. Structure and function changes in the endocrine pancreas of aging rats with reference to the modulating effects of exercise and caloric restriction. J Clin Invest. 68: 75-84. 1981. [Medline] [CrossRef]

Rebuffat P, Belloni AS, Rocco S, Andreis PG, Neri G, Malendowicz LK, Gottardo G, Mazzocchi G, and Nussdorfer GG. The effects of ageing on the morphology and function of the zonae fasciculata and reticularis of the rat adrenal cortex. Cell Tissue Res. 270: 265-272. 1992. [Medline] [CrossRef]

Rehm S, Rapp KG, and Deerberg F. Influence of food restriction and body fat on life span and tumour incidence in female outbred Han:NMRI mice and two sublines. Z Versuchstierkd. 27: 240-283. 1985. [Medline]

Reznik G, and Germann PG. Ganglioneuroma, Adrenal, Rat. In: Monographs on pathology of laboratory animals. Endocrine system. Jones, TC, Capen, $\mathrm{CC}$ and Mohr, U (eds). $2^{\text {nd }}$ ed. Springer, Berlin; New York. pp. 427-432, 1996a.

Reznik G, and Germann PG. Neuroblastoma, Adrenal, Rat. In: Monographs on pathology of laboratory animals. Endocrine system. Jones, TC, Capen, $\mathrm{CC}$ and Mohr, U (eds). 2nd ed. Springer, Berlin; New York. pp. 433-435, 1996b.

Reznik G, Ward JM, and Reznik-Schüller H. Ganglioneuromas in the adrenal medulla of F344 rats. Vet Pathol. 17: 614-621. 1980. [Medline] [CrossRef]

Riley MGI, Boorman GA, and Hayashi Y. Endocrine Pancreas. In: Pathology of the Fischer rat: Reference and atlas. Academic Press, San Diego. pp. 545-553, 1990a.

Riley MGI, Boorman GA, McDonald MM, Longnecker D, Solleveld HA, and Giles HD. Proliferative and Metaplastic Lesions of the Endocrine Pancreas in Rats. In: Guides for Toxicologic Pathology. STP/ARP/AFIP, Washington, DC. pp. 1-7, 1990b.

Robbins SL, and Contran RS. Cellular responses to stress and toxic insults: Adaptation, injury, and death. In: Robbins and Cotran Pathologic Basis of Disease. Robbins, SL, Kumar, V and Cotran, RS (eds). $8^{\text {th }}$ ed. Saunders/Elsevier, Philadelphia. pp. 3-42, 2010a.

Robbins SL, and Contran RS. Tissue Renewal, Regeneration and Repair. In: Robbins and Cotran Pathologic Basis of Disease. Robbins, SL, Kumar, V and Cotran, RS (eds). $8^{\text {th }}$ ed. Saunders/Elsevier, Philadelphia. pp. 79-110, 2010b.

Roe FJ, Lee PN, Conybeare G, Kelly D, Matter B, Prentice D, and Tobin G. The Biosure Study: influence of composition of diet and food consumption on longevity, degenerative diseases and neoplasia in Wistar rats studied for up to 30 months post weaning. Food Chem Toxicol. 33(Suppl 1): 1S-100S. 1995. [Medline] [CrossRef]

Rosol TJ. On-target effects of GLP-1 receptor agonists on thyroid C-cells in rats and mice. Toxicol Pathol. 41: 303-309. 2013. [Medline] [CrossRef]

Rosol TJ, and Capen CC. Tumors of the parathyroid gland and circulating parathyroid hormone-related protein associated with persistent hypercalcemia. Toxicol Pathol. 17: 346-356. 1989. [Medline] [CrossRef]

Rosol TJ, Capen CC, Weisbrode SE, and Horst RL. Humoral hypercalcemia of malignancy in nude mouse model of a canine adenocarcinoma derived from apocrine glands of the anal sac. Biochemical, histomorphometric, and ultrastructural studies. Lab Invest. 54: 679-688. 1986. [Medline]

Rosol TJ, De Lellis RA, Harvey PW, and Sutcliffe C. Endocrine System. In: Haschek and Rousseaux's handbook of toxicologic pathology. Haschek, WM, Rousseaux, CG, Wallig, MA, Bolon, B, Ochoa, R and Mahler, BW (eds). $3^{\text {rd }}$ ed. Academic Press. pp. 2391-2492, 2013.

Rosol TJ, Yarrington JT, Latendresse J, and Capen CC. Adrenal gland: structure, function, and mechanisms of toxicity. Toxicol Pathol. 29: 41-48. 2001. [Medline] [CrossRef]

Rubinstein LJ. Tumors of the Central Nervous System. In: Atlas of Tumor Pathology (II series, fasicle 6). Armed Forces Institute of Pathology, Washington DC. pp. 279-281, 1972.

Russfield AB. Pathology of the endocrine glands, ovary and testis of rats and mice. In: Pathology of laboratory rats and mice. Cotchin, E and Roe, FJC (eds). Blackwell, Oxford; Edinburgh. pp. 391-467, 1967.

Sakai Y, Yamashina S, and Furudate SI. Missing secretory granules, dilated endoplasmic reticulum, and nuclear dislocation in the thyroid gland of rdw rats with hereditary dwarfism. Anat Rec. 259: 60-66. 2000. [Medline] [CrossRef]

Sandusky GE, and Todd GC. Lymphocytic Thyroiditis, Rat. In: Monographs on pathology of laboratory animals. Endocrine system. Jones, TC, Capen, CC and Mohr, U (eds). $2^{\text {nd }}$ ed. Springer, Berlin; New York. pp. 287-290, 1996.

Sass B. Accessory Adrenocortical Tissue, Mouse. In: Monographs on pathology of laboratory animals. Endocrine system. Jones, TC, Capen, CC and Mohr, U (eds). 2nd ed. Springer, Berlin; New York. pp. 391-394, 1996a.

Sass B. Amyloidosis, Adrenal, Mouse. In: Monographs on pathology of laboratory animals. Endocrine system. Jones, TC, Capen, CC and Mohr, U (eds). $2^{\text {nd }}$ ed. Springer, Berlin; New York. pp. 455-458, 1996 b.

Satoh H, Iwata H, Furuhama K, and Enomoto M. Pituicytoma: primary astrocytic tumor of the pars nervosa in aging Fischer 344 rats. Toxicol Pathol. 28: 836-838. 2000. [Medline] [CrossRef]

Schachner M, Huang SK, Ziegelmüller P, Bizzini B, and Taugner R. Glial cells in the pineal gland of mice and rats. A combined immunofluorescence and electron-microscopic study. Cell Tissue Res. 237: 245-252. 1984. [Medline] [CrossRef]

Schaetti P, Argentino-Storino A, Heinrichs M, Mirea D, Popp A, and Karbe E. Aberrant craniopharyngeal structures within the neurohypophysis of rats. Exp Toxicol Pathol. 47: 129-137. 1995. [Medline] [CrossRef]

Seely JC, and Hildebrand PK. Parathyroid Gland. In: Pathology of the Fischer rat: Reference and atlas. Academic Press, San Diego. pp. 537-543, 1990.

Selye H, and Stone H. Hormonally induced transformation of adrenal into myeloid tissue. Am J Pathol. 26: 211-233. 1950. [Medline]

Shimoi A, Kuwayama C, Miyauchi M, Kakinuma C, Kamiya M, Harada T, Ogihara T, Kurokawa M, and Mizuguchi K. Vacuolar changes in the thyroid follicular cells in BrlHan:WIST@Jcl (GALAS) rats.J Toxicol Pathol. 14: 253-257. 2001. [CrossRef]

Shirai M, Kai K, Makino T, Maejima T, Kumagai K, Tanimoto T, Teranishi $\mathrm{M}$, and Sanbuissho A. A spontaneous ganglioneuroma in the adrenal medulla of a young Wistar Hannover rat. Toxicol Pathol. 40: 807-809. 2012. [Medline] [CrossRef]

Solomon A, Weiss DT, Schell M, Hrncic R, Murphy CL, Wall J, McGavin MD, Pan HJ, Kabalka GW, and Paulus MJ. Transgenic mouse model of AA amyloidosis. Am J Pathol. 154: 1267-1272. 1999. [Medline] [CrossRef]

Son WC. Factors contributory to death of young Sprague-Dawley rats in carcinogenicity studies. Toxicol Lett. 153: 213-219. 2004. [Medline] [CrossRef]

Spencer AJ, Andreu M, and Greaves P. Neoplasia and hyperplasia of pancreatic endocrine tissue in the rat: an immunocytochemical study. Vet Pathol. 23: 11-15. 1986. [Medline] [CrossRef]

Steiner DJ, Kim A, Miller K, and Hara M. Pancreatic islet plasticity: interspecies comparison of islet architecture and composition. Islets. 2: 135-145. 
2010. [Medline] [CrossRef]

Strandberg JD. Adenocarcinoma, Adrenal Cortex, Rat. In: Monographs on pathology of laboratory animals. Endocrine system. Jones, TC, Capen, $\mathrm{CC}$ and Mohr, U (eds). $2^{\text {nd }}$ ed. Springer, Berlin; New York. pp. 444-448, 1996a.

Strandberg JD. Adenoma, Adrenal Cortex, Rat. In: Monographs on pathology of laboratory animals. Endocrine system. Jones, TC, Capen, CC and Mohr, U (eds). $2^{\text {nd }}$ ed. Springer, Berlin; New York. pp. 440-444, 1996b.

Strandberg JD. Focal Hyperplasia, Adrenal Cortex, Rat. In: Monographs on pathology of laboratory animals. Endocrine system. Jones, TC, Capen, $\mathrm{CC}$ and Mohr, U (eds). $2^{\text {nd }}$ ed. Springer, Berlin; New York. pp. 435-439, $1996 \mathrm{c}$.

Strandberg JD. Hyperplasia and Pheocromocytoma, Adrenal Medulla, Rat. In: Monographs on pathology of laboratory animals. Endocrine system. Jones, TC, Capen, CC and Mohr, U (eds). $2^{\text {nd }}$ ed. Springer, Berlin; New York. pp. 411-421, 1996d.

Stromberg PC, Wilson F, and Capen CC. Immunocytochemical demonstration of insulin in spontaneous pancreatic islet cell tumors of Fischer rats. Vet Pathol. 20: 291-297. 1983. [Medline] [CrossRef]

Tajima K, Miyagawa J, Nakajima H, Shimizu M, Katayama S, Mashita K, and Tarui S. Morphological and biochemical studies on minocycline-induced black thyroid in rats. Toxicol Appl Pharmacol. 81: 393-400. 1985. [Medline] [CrossRef]

Takaoka M, Yamoto T, Teranishi M, Manabe S, and Goto N. A case of intracytoplasmic edema of follicular epithelial cells in rat thyroid. $J$ Vet Med Sci. 56: 989-991. 1994. [Medline] [CrossRef]

Tekeli S, Morton D, and Cusick PK. Pituicytoma in a mouse. Toxicol Pathol. 25: 516-517. 1997. [Medline] [CrossRef]

Teta M, Long SY, Wartschow LM, Rankin MM, and Kushner JA. Very slow turnover of beta-cells in aged adult mice. Diabetes. 54: 2557-2567. 2005. [Medline] [CrossRef]

Theuring F, Götz W, Balling R, Korf HW, Schulze F, Herken R, and Gruss P. Tumorigenesis and eye abnormalities in transgenic mice expressing MSV-SV40 large T-antigen. Oncogene. 5: 225-232. 1990. [Medline]

Tischler AS. Histology of the paraganglia. In: Histology for pathologists. Sternberg, SS (ed) Raven Press, New York. pp. 363-380, 1992.

Tischler AS. Cell Proliferation in the Adult Adrenal Medulla: Chromaffin Cells as a Model for Indirect Carcinogenesis. In: Monographs on pathology of laboratory animals. Endocrine system. Jones, TC, Capen, CC and Mohr, U (eds). 2nd ed. Springer, Berlin; New York. pp. 405-411, 1996.

Tischler AS, and Coupland RE. Changes in structure and function of the adrenal medulla. In: Pathobiology of the aging rat. Mohr, U, Dungworth, DL and Capen, CC (eds). International Life Sciences Institute, Washington, D.C. pp. 245-268, 1994.

Tischler AS, Ruzicka LA, Van Pelt CS, and Sandusky GE. Catecholaminesynthesizing enzymes and chromogranin proteins in drug-induced proliferative lesions of the rat adrenal medulla. Lab Invest. 63: 44-51. 1990. [Medline]

Todd GC. Induction and reversibility of thyroid proliferative changes in rats given an antithyroid compound. Vet Pathol. 23: 110-117. 1986. [Medline] [CrossRef]

Tomonari Y, Sato J, Wako Y, and Tsuchitani M. Age-related histological findings in the pineal gland of $\mathrm{Crl}: \mathrm{CD}(\mathrm{SD})$ rats. J Toxicol Pathol. 25: 287-291. 2012. [Medline] [CrossRef]

Treumann S, Buesen R, Gröters S, Eichler JO, and van Ravenzwaay B. Occurrence of pineal gland tumors in combined chronic toxicity/carcinogenicity studies in Wistar rats. Toxicol Pathol. 43: 838-843. 2015. [Medline] [CrossRef]

Tucker MJ. Adrenal Gland. The Endocrine System. In: Diseases of the Wistar rat. Taylor \& Francis, London; Bristol, PA. pp. 193-201, 1997.

Unger PD, Cohen JM, Thung SN, Gordon R, Pertsemlidis D, and Dikman SH. Lipid degeneration in a pheochromocytoma histologically mimicking an adrenal cortical tumor. Arch Pathol Lab Med. 114: 892-894. 1990. [Medline]

Van Zwieten MJ, Frith CH, Nooteboom AL, Wolfe HJ, and Delellis RA. Medullary thyroid carcinoma in female BALB/c mice. A report of 3 cases with ultrastructural, immunohistochemical, and transplantation data. $\mathrm{Am}$
J Pathol. 110: 219-229. 1983. [Medline]

Vazquez R, Carretero J, Blanco E, and Carvajal JC. Morphology of the neural lobe of the hypophysis in rats treated with furosemide. II. Pituicytes. Acta Anat (Basel). 128: 171-176. 1987. [Medline] [CrossRef]

Venalis A, Bradunaite R, Vaitkiene D, Povilenaite D, and Leonaviciene L. Experimental amyloidosis induced by Freund's ajuvant and caseine in two mouse strains. In: Amyloid and amyloidosis. Skinner, M, Grateau, G and Kyle, RA (eds). CRC Press, Boca Raton, FL. pp. 231, 2005.

Vogel H, and Fuller GN. Primitive neuroectodermal tumors, embryonal tumors, and other small cell and poorly differentiated malignant neoplasms of the central and peripheral nervous systems. Ann Diagn Pathol. 7: 387398. 2003. [Medline] [CrossRef]

Walker VE, Morgan KT, Zimmerman HM, and Innes JRM. Tumours of the central and peripheral nervous system. In: Pathology of tumours in laboratory animals Volume 2: Tumours of the mouse. Turusov, VS and Mohr, $\mathrm{U}$ (eds). $2^{\text {nd }}$ ed. International Agency for Research on Cancer; Oxford University Press, Lyon; New York. pp. 731-776, 1994.

Wallace MJ, Batt J, Fladd CA, Henderson JT, Skarnes W, and Rotin D. Neuronal defects and posterior pituitary hypoplasia in mice lacking the receptor tyrosine phosphatase PTPsigma. Nat Genet. 21: 334-338. 1999. [Medline] [CrossRef]

Ward JM, Goodman DG, Squire RA, Chu KC, and Linhart MS. Neoplastic and nonneoplastic lesions in aging (C57BL/6N x C3H/HeN)F1 (B6C3F1) mice. J Natl Cancer Inst. 63: 849-854. 1979a. [Medline] [CrossRef]

Ward JM, and Ohshima M. The role of iodine in carcinogenesis. Adv Exp Med Biol. 206: 529-542. 1986. [Medline]

Ward JM, and Reznik-Schüller H. Morphological and histochemical characteristics of pigments in aging F344 rats. Vet Pathol. 17: 678-685. 1980. [Medline] [CrossRef]

Ward JM, Stinson SF, Hardisty JF, Cockrell BY, and Hayden DW. Neoplasms and pigmentation of thyroid glands in F344 rats exposed to 2,4-diaminoanisole sulfate, a hair dye component. J Natl Cancer Inst. 62: 1067-1073. 1979b. [Medline]

Warren S, Grozdev L, Gates O, and Chute RN. Radiation-induced adrenal medullary tumors in the rat. Arch Pathol. 82: 115-118. 1966. [Medline]

Weber K, Ernst R, Fankhauser H, Hardisty JF, Heider W, and Stevens K. Thyroid dysplasia in Wistar Hannover GALAS rats. $J$ Toxicol Pathol. 22: 247-254. 2009. [Medline] [CrossRef]

Wei XY, Zhao CH, Liu YY, Wang YZ, and Ju G. Immuohistochemical markers for pituicyte. Neurosci Lett. 465: 27-30. 2009. [Medline] [CrossRef]

Wild P, and Setoguti T. Mammalian parathyroids: morphological and functional implications. Microsc Res Tech. 32: 120-128. 1995. [Medline] [CrossRef]

Wong WP, Scott DW, Chuang CL, Zhang S, Liu H, Ferreira A, Saafi EL, Choong YS, and Cooper GJ. Spontaneous diabetes in hemizygous human amylin transgenic mice that developed neither islet amyloid nor peripheral insulin resistance. Diabetes. 57: 2737-2744. 2008. [Medline] [CrossRef]

Wright JA, Wadsworth PF, and Stewart MG. Neuron-specific enolase immunoreactivity in rat and mouse phaeochromocytomas. J Comp Pathol. 102: 475-478. 1990. [Medline] [CrossRef]

Yamakawa S, Iwata H, Hirouchi Y, Kobayashi K, and Enomoto M. Pancreatic hepatocytes in $\mathrm{B} 6 \mathrm{C} 3 \mathrm{~F} 1(\mathrm{C} 57 \mathrm{BL} / 6 \mathrm{CrSlc} \times \mathrm{C} 3 \mathrm{H} / \mathrm{HeSlc})$ and $\mathrm{BDF} 1$ (C57BL/6NCrj x DBA/2NCrj) mice. J Toxicol Pathol. 8: 117-121. 1995. [CrossRef]

Yamamoto O, Mitsumori K, Yoshida T, Maita K, and Shirasu Y. Spontaneous malignant pineocytoma in a female Wistar rat. J Vet Med Sci. 53: 527-529. 1991. [Medline] [CrossRef]

Yamaoka T, Idehara C, Yano M, Matsushita T, Yamada T, Ii S, Moritani M, Hata J, Sugino H, Noji S, and Itakura M. Hypoplasia of pancreatic islets in transgenic mice expressing activin receptor mutants. J Clin Invest. 102: 294-301. 1998. [Medline] [CrossRef]

Yamazaki M, Kato A, Kato C, Fujii E, Adachi K, Miyoshi A, and Suzuki M. Segmentation of the pathophysiological stages of diabetic changes in the db/db mouse. J Toxicol Pathol. 22: 133-137. 2009. [Medline] [CrossRef]

Yarrington JT. Adrenal Cortex. In: Pathobiology of the Aging Mouse. Mohr, U, Dungworth, DL, Capen, CC, Carlton, WW, Sundberg, JP and Ward, JM (eds). ILSI, Washington, DC. pp. 125-133, 1996. 
Yarrington JT, and O'Neal Johnston J. Aging in the adrenal cortex. In: Pathobiology of the aging rat. Mohr, U, Dungworth, DL and Capen, CC (eds). International Life Sciences Institute, Washington, D.C. pp. 227-244, 1992.

Yasui Y, Ohta Y, Ueda Y, Hasegawa K, Kihara T, Hosoi M, Miyajima R, Shiga A, Imai K, and Toyoda K. Spontaneous ganglioneuroma possibly originating from the trigeminal ganglion in a $\mathrm{B} 6 \mathrm{C} 3 \mathrm{~F} 1$ mouse. Toxicol Pathol. 37: 343-347. 2009. [Medline] [CrossRef]

Yasui Y, Ueda Y, Nagai K, Yamashita R, Ishizaki M, Kihara T, Hasegawa K, Hosoi M, Miyajima R, Shiga A, Iwata A, and Imai K. Spontaneous pituitary carcinoma of the pars intermedia in $\mathrm{B} 6 \mathrm{C} 3 \mathrm{~F} 1$ mouse. $J$ Toxicol Pathol. 21: 105-108. 2008. [CrossRef]

Yasuno H, Watanabe T, Miyamoto Y, Kandori H, Yamasaki H, and Fukuda R. Expression of steroidogenic factor 1 and pituitary specific transcription factor 1 in rat pituitary adenomas. J Toxicol Pathol. 26: 209-213. 2013.
[Medline] [CrossRef]

Yoshizawa K, Walker NJ, Nyska A, Kissling GE, Jokinen MP, Brix AE, Sells DM, and Wyde ME. Thyroid follicular lesions induced by oral treatment for 2 years with 2,3,7,8-tetrachlorodibenzo-p-dioxin and dioxin-like compounds in female Harlan Sprague-Dawley rats. Toxicol Pathol. 38: 1037-1050. 2010. [Medline] [CrossRef]

Zabka TS, Fielden MR, Garrido R, Tao J, Fretland AJ, Fretland JL, Albassam MA, Singer T, and Kolaja KL. Characterization of xenobiotic-induced hepatocellular enzyme induction in rats: anticipated thyroid effects and unique pituitary gland findings. Toxicol Pathol. 39: 664-677. 2011. [Medline] [CrossRef]

Zwicker GM, and Eyster RC. Chronic effects of corticosteroid oral treatment in rats on blood glucose and serum insulin levels, pancreatic islet morphology, and immunostaining characteristics. Toxicol Pathol. 21: 502508. 1993. [Medline] [CrossRef] 